\title{
Pursuing High-Value, Cost-Conscious Care
}

Citation for published version (APA):

Stammen, L. (2020). Pursuing High-Value, Cost-Conscious Care: The Role of Medical Education.

[Doctoral Thesis, Maastricht University]. Maastricht University. https://doi.org/10.26481/dis.20201016ls

Document status and date:

Published: 01/01/2020

DOI:

10.26481/dis.20201016ls

Document Version:

Publisher's PDF, also known as Version of record

\section{Please check the document version of this publication:}

- A submitted manuscript is the version of the article upon submission and before peer-review. There can be important differences between the submitted version and the official published version of record.

People interested in the research are advised to contact the author for the final version of the publication, or visit the DOI to the publisher's website.

- The final author version and the galley proof are versions of the publication after peer review.

- The final published version features the final layout of the paper including the volume, issue and page numbers.

Link to publication

\footnotetext{
General rights rights.

- You may freely distribute the URL identifying the publication in the public portal. please follow below link for the End User Agreement:

www.umlib.nl/taverne-license

Take down policy

If you believe that this document breaches copyright please contact us at:

repository@maastrichtuniversity.nl

providing details and we will investigate your claim.
}

Copyright and moral rights for the publications made accessible in the public portal are retained by the authors and/or other copyright owners and it is a condition of accessing publications that users recognise and abide by the legal requirements associated with these

- Users may download and print one copy of any publication from the public portal for the purpose of private study or research.

- You may not further distribute the material or use it for any profit-making activity or commercial gain

If the publication is distributed under the terms of Article $25 \mathrm{fa}$ of the Dutch Copyright Act, indicated by the "Taverne" license above, 
Pursuing High-Value, Cost-Conscious Care The Role of Medical Education 
The research reported here was carried out at

\section{U. Maastricht University}

Maastricht UMC+

in the School of Health Professions Education

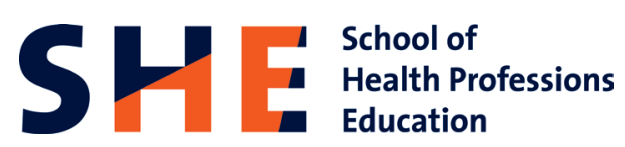

(C) Lorette Stammen, 2020, Maastricht

ISBN 978-94-640-2508-8

Cover: Gianluca Riggio,Vagebond

Printed by: Ipskamp Printing

Printed with financial support from Maastricht University and SBO

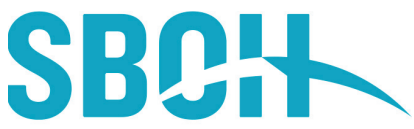

voor artsen in opleiding 


\section{Pursuing}

\section{High-Value, \\ Cost-Conscious \\ Care}

\section{The Role of Medical Education}

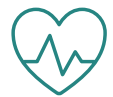

\section{DISSERTATION}

to obtain the degree of Doctor

at the Maastricht University,

on the authority of the Rector Magnificus,

Prof.dr. Rianne M. Letschert

in accordance with the decision

of the Board of Deans,

to be defended in public

on Friday October I6th, 2020, I2:00 hours

by

Lorette Stammen 
Supervisor

Prof. Dr. Laurents Stassen

Prof. Dr. Erik Driessen

Prof. Dr. Fedde Scheele

\author{
Co-supervisor \\ Dr. Renée Stalmeijer
}

Assessment committee

Prof. Dr. Walther van Mook (chair)

Prof. Dr. Frank Smeenk

Prof. Dr. Debbie Jaarsma

Prof. Dr. Jan Kremer

Dr. Jesse Jansen 


\section{Table of Contents}

$\begin{array}{lll}\text { Chapter One } & \text { General Introduction }\end{array}$

Chapter Two Training Physicians to Provide High-value,

Cost-conscious Care:A Systematic Review $\quad 18$

Chapter Three The Struggle is Real - How Residents Learn to Provide

High-Value, Cost-Conscious Care

Chapter Four How Do Attending Physicians Prepare Residents

to Deliver High-Value, Cost-Conscious Care? 90

Chapter Five A wealth of opportunities: an ethnographic study on learning to deliver high-value, cost-conscious care

Chapter Six

12 Tips voor het opleiden in doelmatigheid van zorg.

General Discussion

Summary

Samenvatting

Valorization

About the author

SHE Dissertation series 


\section{Chapter I}

General Introduction 


\section{Introduction - Sustainable health care systems}

The traditional research field of medical education concerns issues like curriculum design, teaching methods, mentoring, assessment methods and faculty development. Now that this field has been developed to a relatively high level of theory building and understanding, a new kind of research question is emerging: how to use current knowledge about medical education for urgent societal issues like patient centeredness, dealing with complex health organizations and last, but not least, the care for oneself as a professional in a highly demanding environment. ${ }^{1}$ The CanMEDs 2015 report is one of the replies of the field of medical education to deal with this fundamental new way of approaching medical education. ${ }^{2}$ This new approach to medical education seems to move the field of medical education beyond the perspective that the new health care professional is merely a medical expert or a member of a professional team or network, but functions as a change agent. ${ }^{3}$ Change agents have developed leadership attributes and are capable of transferring core competencies to different settings. These core competencies focus on searching information and, though analysis and synthesis, using this information for clinical decision-making. Also, change agents can adapt to local circumstances and work effectively within health care teams. Change agents are the product of transformative learning. ${ }^{3}$ However, defining the training of a change agent is not easy. The change agent is responsible for attaining the prerequisites of highquality, sustainable health systems: acceptable, accessible, equitable, and affordable delivery of high-quality care. ${ }^{4}$ This dissertation aims to contribute to the development of knowledge required for training physicians in their role as change agents. The focus is on the grassroots level: the provision of high-value, cost-conscious care (HV3C) by the medical professional.

\section{Problem statement}

\section{I Rising health care costs}

Healthcare costs increasingly weigh on governmental expenditure. ${ }^{5}$ The gross domestic product (GDP) percentage expresses the amount of money spent on health care services, which has increased considerably worldwide in the last decade. ${ }^{5}$ Rising health care costs are caused by aging population, prosperity, technological advancement, fear of legislation and increasing patient demands. ${ }^{6,7}$ The hazards of spending substantial parts of national budgets on health care needs consideration. First, governmental money spent on health care cannot be spent on other compelling and worthy social areas such as education, environment, infrastructure and defense, areas which also influence (economic) value and contribute to general health and wellbeing. ${ }^{8,9}$ Second, citizens will inevitability and increasingly bear the costs of care through insurance policies and tax. Increasing out-of-pocket spending will influence health care accessibility and equity. Since the literature states that $60 \%$ of health care expenditure is influenced by physicians, ${ }^{10,11}$ they are an important subject for intervention. 


\section{Pursuing High-Value, Cost-Conscious Care}

\subsection{Health care quality}

In contrast to the rapid increase in health care costs is the slow improvement in quality in recent decades. ${ }^{5}$ In addition, health care is criticized for lacking humanity, empathy, and issues regarding overtreatment and overdiagnosis. ${ }^{12-15}$ Estimates indicate that $20 \%$ of health care costs are considered wasteful, meaning that they do not result in health benefit. ${ }^{10,16}$ Another important critique regarding quality of health care is the simplistic approach taken by those aiming to evaluate quality. What can be considered 'good' care remains one of the biggest challenges in both care delivery and science as it is multi-factorial and depends on both patient and contextual factors. ${ }^{17}$ In addition, little is known about the burden of treatment ${ }^{18}$ that patients face. In line with the WHO definition of health: "the state of complete physical, mental and social wellbeing and not merely the absence of diseases or infirmity", there is increased attention for the role of physicians in guiding patients to adapt to and self-manage their health. ${ }^{19,20}$ Unfortunately, quality measures more often than not focus on quantifiable data such as morbidity and mortality rates and do not include measurements such as burden of disease or burden of treatment. Despite the limitations of outcome measurements, it is important to gather information on quality of care delivery which can be used for benchmarking and detection of best practices. On a higher level, measurements can be used to compare care delivery on regional, national and international levels and to provide feedback to responsible management for care delivery. ${ }^{19}$

\section{The two main actors in this dissertation}

The delivery of high-value, cost-conscious care in this dissertation knows two main actors; the consumers who might have been, are, or will be patients, and the providers (health care professionals). Since physicians play an important role in medical decision-making, our research focused primarily on the role of physicians. We will discuss their role in health care costs and quality in the following sections.

\section{I The role of patients in care delivery}

Citizens determine the demand for health care and directly influence the use of health care resources. Through various pathways citizens pay directly or indirectly for the costs of health care and medical training. The medical profession and medical education are expected to meet societal demands and expectations of what can be considered good care delivery. The acknowledgment of the important role of patients, together with the increasing verbalization of their preferences in the past 50 years has led to a shift in the care delivery model from 
physician-oriented (paternalism) to patient-oriented (patient-centered) care models. ${ }^{21}$ Patientcentered care is defined by the Institute of Medicine as "care that is respectful of and responsive to individual patient preferences, needs, and values" ${ }^{22-24}$ Therefore, when citizens seek health care, they play an important role in determining which care they receive. When care delivery does not consider the patient's preferences, their contextual factors and abilities, it is challenging to let them contribute to their recovery, therapeutic successes, health or self-management of their disease. $^{25}$

\subsection{The role of physicians in care delivery}

Although many Western countries score high on quality of care, it is questionable if all the money involved is well spent. ${ }^{8,10}$ Examples of debatable care that is often considered wasteful are extensive laboratory testing (e.g. a blood test before seeing the patient), routinely/daily testing of admitted patients (daily ratios), the use of outdated imaging techniques (abdominal $\mathrm{x}$-ray for obstipation or aspecific abdominal complaints), and invasive procedures without taking time for spontaneous recovery (hernia surgery within six months). Wasteful treatments are worrying since health care services are expensive and contribute to the burden of disease, increased emotional stress for patients, may expose them to radiation and side effects which are generally not without harm. Due to the rising number of available diagnostic services, treatment options and health care costs, together with increasing expectations and patients' articulateness, there is an urge for a more deliberate approach to answering the question 'which care is appropriate?'. Physicians can be held accountable for health care delivery on the individual and societal level. Since current figures estimate $20 \%$ of health care services as wasteful, meaning that they do not add value for patients, ${ }^{8,10,23}$ physicians remain an important point of interest in the delivery of appropriate care.

Physicians' care delivery largely stems from the formation of their professional identity during undergraduate and postgraduate medical training. ${ }^{26}$ During their training they develop from lay person into a medical expert, possessing all the skills, knowledge and attitudes that are deemed essential. Competency frameworks that are fundamental to residency training increasingly call for training in medical leadership, stewardship of resources, and systems practice in both undergraduate and postgraduate training. ${ }^{2,27,28}$ This dissertation aims to unravel how medical education can support the role of physicians in the affordability of health care, while safeguarding quality. 


\section{Theoretical background}

\section{I High-value, cost-conscious care and related concepts}

Balancing health care costs and quality of care has gained attention in recent decades. ${ }^{16,17,29-31}$ Several concepts are used to identify the pursuit of high-quality care delivery while slowing down health care expenditure. High-value, cost-conscious care (HV3C) is the process of balancing the benefits, risks and costs of health care services, with the purpose to deliver care that adds value. ${ }^{30,32}$ Weinberger described HV3C in 2011 in his call to train physicians in a critical seventh competence; high-value, cost-conscious care (HV3C). ${ }^{30}$ The concepts concerning $\mathrm{HV} 3 \mathrm{C}$ hail from diverse perspectives, ranging from health economics to ethics, but all share the collective aim of increasing value and reducing costs. Table 1 presents an overview of HV3C-related concepts and their backgrounds. We choose to use HV3C throughout this dissertation since this concept is commonly used in medical education literature and explicitly mentions the importance of both cost and quality in care delivery.

Table I: Overview of HV3C-related concepts

\begin{tabular}{|c|c|c|c|c|}
\hline & Abbreviation & Year & Founder & Aim \\
\hline $\begin{array}{l}\text { High-value, cost- } \\
\text { conscious care }\end{array}$ & $\mathrm{HV} 3 \mathrm{C}$ & 2011 & Weinberger ${ }^{30}$ & $\begin{array}{l}\text { Deliver care that adds value for patients, and } \\
\text { balances benefits, risks and costs. }\end{array}$ \\
\hline Value-based healthcare & VBHC & 2006 & Porter $^{17}$ & $\begin{array}{l}\text { Increase value for patients while reducing } \\
\text { health care costs. }\end{array}$ \\
\hline Value-based medicine & VBM & 2003 & Brown $^{33}$ & $\begin{array}{l}\text { Medicine based upon the patient-perceived } \\
\text { value conferred by an intervention. }\end{array}$ \\
\hline Choosing wisely & & 2012 & Levinson $^{34}$ & $\begin{array}{l}\text { Advance a national dialogue on avoiding } \\
\text { unnecessary medical tests, treatments and } \\
\text { procedures. }\end{array}$ \\
\hline Triple aim & & 2008 & Berwick $^{29}$ & $\begin{array}{l}\text { Improve the experience of care, improve the } \\
\text { health of populations and reducing the per } \\
\text { capita costs of care. } \\
\text { Better health, better health care, and lower } \\
\text { costs. }\end{array}$ \\
\hline
\end{tabular}




\subsection{Training for high-value, cost-conscious care delivery}

Over the last two decades much effort is put into training future physicians in support of their role in delivering HV3C., 27,28,35 Many interventions aim to change physician behavior, based on the theory of planned behavior, ${ }^{36}$ which states that in order to deliver HV3C, physicians should first intend to behave accordingly. ${ }^{37}$ Insight in individual attitudes regarding $\mathrm{HV} 3 \mathrm{C}$ is valuable since interventions often aim to positively influence the attitude, beliefs and perceived control to deliver HV3C. Such attitudes can be measured through the Maastricht HVCCC Attitude Questionnaire (MHAQ). ${ }^{38}$ Other commonly used interventions build on cognitivist learning principles and the assumption that filling a knowledge gap will lead to more considerate use of resources. ${ }^{37,39}$ These approaches focus on providing knowledge about the cost of either services or guidelines. Research into the effects of these approaches focuses primarily on evaluating the short-term effects of educational interventions through a pre-post-intervention design. This design, combined with the rare use of control groups, makes it difficult to move beyond the conclusion that education can support the training of physicians in HV3C. However, these studies do not explore in detail which elements of training contribute to the effect of these interventions. Additionally, it is insufficiently known how educational interventions can help prepare physicians for their role in $\mathrm{HV} 3 \mathrm{C}$ delivery, and indeed in how future physicians learn.

\subsection{Medical education continuum}

\section{Undergraduate and postgraduate training}

Physicians are often the focus of programs and interventions aimed at containing costs and/or improving quality of care. Physicians' behavior is partly shaped by the training they receive, both formal and informal. ${ }^{40-42}$ Physicians' training and the medical education continuum share features across the world. After admittance to the school of medicine, medical students join an undergraduate training program. This usually lasts $4-6$ years, is often based on competency frameworks, e.g. the Canadian Medical Education Directives for Specialists (CanMEDS), and the Accreditation Council for Graduate Medical Education (ACGME) and focuses on basic and clinical sciences and skills training. ${ }^{2,27}$ After completing the undergraduate program, medical students receive their medical degree and may apply to a postgraduate training program. These programs are largely situated in workplace settings (i.e. hospitals, public and primary care centers) and train residents in their medical field of interest to help them become a medical specialist. In workplace settings residents receive both formal and informal training while caring for patients. Therein lies an important role for attending physicians to provide guidance and supervision to residents. At the end of residency training, residents are expected to be ready for independent, unsupervised practice. 


\section{Pursuing High-Value, Cost-Conscious Care}

\section{Formal education, workplace-based learning and the hidden curriculum}

In this dissertation we use the term 'formal training' to describe training that occurs within an organizational context that is deliberately designed for learning, e.g. a class room, simulation center, training institute. ${ }^{43}$ This type of education is structured, meaning there are pre-set learning objectives and activities. The workplace, or the workplace-based learning environment is where 'patients, learners and practitioners come together for the conjoint purpose of medical care and learning. ${ }^{43}$ This environment is important since it shapes the habits, behavior, attitudes and values of the learners present within it. ${ }^{44}$ Clinical supervisors who are (more) senior members of the medical staff are known to fulfill an important role in the workplace-based environment by facilitating the resident's learning and development while guarding patient safety. ${ }^{45}$ The workplace-based setting is also where normative rules regarding physician behavior and responsibilities are transferred from one person to another. These learned normative rules are part of the hidden curriculum and are known to be essential for the development of the professional identity. The hidden curriculum encompasses all lessons learned that are not described in the formal curriculum and are transferred through the process of socialization. By definition, socialization is 'the processes by which people acquire the values and attitudes, the interest, skills and knowledge - in short, the culture - current in the groups of which they are, or seek to become, a member. ${ }^{21}$ Medical students and residents are taught what is valued by the current generation of specialists by observing their behavior, attitudes, line of reasoning and general discourse. Especially ethics, rationales, clinical responsibility, together with dealing with uncertainty and emotions are examples of topics that the hidden curriculum primarily addresses.

\section{Aim of this dissertation}

Given the importance of high-value, cost-conscious care for the sustainability of health care and the essential role of (future) physicians in this endeavor, this dissertation investigates the role of medical education in the training of high-value, cost-conscious care delivery using the following research questions:

1. Which elements of educational interventions are effective to train (future) physicians in the delivery of high-value, cost-conscious care?

2. How do residents approach the delivery of high-value, cost-conscious care in daily practice?

3. How do supervisors aim to train residents in the delivery of high-value, cost-conscious care?

4. How is the workplace setting related to residents' high-value, cost-conscious care delivery? 
Table 2: Overview of studies in this dissertation

\begin{tabular}{|c|c|c|c|}
\hline Chapter & Research question & Approach & Participants \\
\hline Chapter 2 & $\begin{array}{l}\text { How and under what circumstances } \\
\text { may educational interventions help } \\
\text { practicing physicians, residents in } \\
\text { training and medical students to } \\
\text { provide high-value, cost-conscious } \\
\text { care? }\end{array}$ & $\begin{array}{l}\text { Qualitative/Quantitative:A } \\
\text { systematic realist review }\end{array}$ & \\
\hline Chapter 3 & $\begin{array}{l}\text { a) How do residents learn to } \\
\text { provide high-value, cost- } \\
\text { conscious care in the workplace } \\
\text { setting? } \\
\text { b) How does the workplace } \\
\text { influence this learning? }\end{array}$ & $\begin{array}{l}\text { Qualitative/Focus group } \\
\text { research }\end{array}$ & Residents \\
\hline Chapter 4 & $\begin{array}{l}\text { How do supervisors aim to prepare } \\
\text { residents to deliver high-value, cost- } \\
\text { conscious care? }\end{array}$ & $\begin{array}{l}\text { Qualitative/Semi-structured } \\
\text { interviews }\end{array}$ & Attending physicians \\
\hline Chapter 5 & $\begin{array}{l}\text { How is the workplace setting related } \\
\text { to residents' high-value, cost- } \\
\text { conscious care delivery? }\end{array}$ & Qualitative/Ethnography & $\begin{array}{l}\text { Residents and all health care } \\
\text { professionals present in } \\
\text { their workplace }\end{array}$ \\
\hline Chapter 6 & $\begin{array}{l}\text { Training in HV3C delivery:Tips and } \\
\text { tricks for clinical practice. }\end{array}$ & Summary & \\
\hline
\end{tabular}

\section{Positionality statement and reflexivity}

I have been undergoing postgraduate medical training while researching medical education, a combination that has created interesting opportunities in both fields. It offered me the chance to experience what residency training entails, and how difficult it can be to juggle all the stakes: patient care, personal development as a physician, and being a health care team member. My experiences as a resident therefore influenced the research I conducted, including the questions asked, the approaches taken, the way we collected and interpreted data, what we chose to discuss and where we aimed to publish. My research focuses on how people learn, both formally and informally. It is based on the belief that knowledge is constructed based on information offered and peoples' own understanding of that information. Therefore, what is learned is not the same for everybody and is strongly influenced by prior knowledge and the personal experience of the learner or researcher. This belief is captured in the social constructivist paradigm. For our research, this also entailed trying to understand the way physicians learn, rather than 'discovering' the truth about learning.

I began my research in the summer of 2013 shortly after receiving my medical degree. I had spent my final year of undergraduate training studying the competence of leadership in the 


\section{Pursuing High-Value, Cost-Conscious Care}

field of medical education at the Atrium hospital Heerlen (nowadays the Zuyderland hospital). After working as a student-assistant for over four years in the Department of Educational Research and Development ('O\&O') at Maastricht University, it was obvious to me that if I started a PhD project, it would be in this department that had familiarized me with medical education and with the person who had introduced me to research (Renée Stalmeijer). Fulltime research was never my ambition, and early in the $\mathrm{PhD}$ project, we discussed the option to combine research with clinical training. After being selected for the family medicine residency program in 2015, I worked part-time in research and part-time in clinical practice. Being a resident helped me to gain access to potential participants, both residents and clinicians, and gave me the ability to speak the same language as the subjects of my research. Doing research in a field you think you are familiar with entails the risk that you 'assume' you know what is going on, and things might appear so obvious that it can be difficult to see what is actually happening. Therefore, working in a research team with both educational researchers and clinical educators was a necessity. I changed the composition of our team for the different studies, to expose myself to different perspectives. Nevertheless, being a resident helped me pose critical questions to the participants, who understood that my questions did not derive from a judgment, but from the aim to improve the way care is delivered to our patients and improve the affordability of health care.

Finding the right 'tone' was challenging when I was writing the articles included in this dissertation. Describing how residents sometimes avoid learning opportunities, prioritize their personal goals above HV3C delivery, or simply choose the path of least resistance easily sounds like something 'wrong' or 'undesirable'. Working in clinical practice, I experienced that finishing consultations within the allocated time, keeping patients and supervisors satisfied, and naively searching for certainty are all very present. Just as any other resident, I feel these are all challenges and I also struggle to balance my ideals with simply getting through the hectic day. There is a thin line between being critical and judgmental, and at times I felt our published articles fell short in describing the passion and dedication participants showed during our data collection period. Keeping up the image of residents as the hard-working, devoted physicians I know they are, while at the same time identifying areas for improvement in HV3C delivery has been the biggest challenge of my PhD trajectory.

\section{Overview of studies in this dissertation}

This dissertation aims to unravel the role of medical education in the delivery of high-value, costconscious care. We specifically aimed to explore how residents learn to deliver high-value, costconscious care. Chapter 2 provides a realist synthesis of educational interventions that aim to train medical students, residents and physicians in the delivery of high-value, cost-conscious care, aiming to further unravel what can be considered as 'active ingredients' of formal education. 
Chapter 3 focuses on residents' perceptions of high-value, cost-conscious care training in the workplace. How are they being trained and how can the learning environment support workplacebased learning? Supervisors are key figures in Chapter 4, exploring how they aim to prepare residents for the delivery of high-value, cost-conscious care. In Chapter 5, we aim to unravel how the learning environment relates to the delivery of high-value, cost-conscious care by residents. We chose to observe residents during clinical care delivery in daily practice. In order to help develop training in daily practice, we summarized our findings from this dissertation and generated tips for both attending physicians and resident to utilize $\mathrm{HV} 3 \mathrm{C}$ training in daily practice. These tips are described in Chapter 6. In Chapter 7, we discuss the coherence between chapters and aim to place the findings in a larger perspective.

\section{References}

1. van der Aa JE, Scheele F, Goverde AJ, Teunissen PW. A qualitative study on harmonization of postgraduate medical education in Europe: negotiating flexibility is key. Perspect Med Educ. 2019;8(4):216-222.

2. Canada RCoPaSo. CanMEDS 2015 Physician Competency Framework. www.canmeds.royalcollege.ca: Frank JR, Snell L, Sherbino J, editors. CanMEDS 2015 Physician Competency Framework. Ottawa: Royal College of Physicians and Surgeons of Canada; 2015.;2015.

3. Frenk J, Chen L, Bhutta ZA, et al. Health professionals for a new century: transforming education to strengthen health systems in an interdependent world. Lancet. 2010;376(9756):1923-1958.

4. Toebes. The right to health care is a human right. 1999.

5. WHO. https://apps.who.int/nha/database/Select/Indicators/en. Published 2018. Accessed 12-12-2019, 2019.

6. Blumenthal D. Controlling health care expenditures. N Engl J Med. 2001;344(10):766-769.

7. Ginsburg PB. Controlling health care costs. N EnglJ Med. 2004;351(16):1591-1593.

8. Berwick DM, Hackbarth AD. Eliminating waste in US health care. JAMA. 2012;307(14):1513-1516.

9. Schroeder SA, Zones JS, Showstack JA. Academic medicine as a public trust. JAMA. 1989;262(6):803-812.

10. Agrawal S, Taitsman J, Cassel C. Educating physicians about responsible management of finite resources. JAMA. 2013;309(11):1115-1116.

11. Crosson FJ. Change the microenvironment. Delivery system reform essential to control costs. Mod Healthc. 2009;39(17):20-21.

12. Peabody FW. The care of the patient. JAMA. 2015;313(18):1868.

13. Pedersen R. Empathy development in medical education--a critical review. Med Teach. 2010;32(7):593-600.

14. Brody H. Medicine's ethical responsibility for health care reform--the Top Five list. NEnglJ Med. 2010;362(4):283-285.

15. Moynihan R, Doust J, Henry D. Preventing overdiagnosis: how to stop harming the healthy. BMJ. 2012;344:e3502.

16. Born K, Kool T, Levinson W. Reducing overuse in healthcare: advancing Choosing Wisely. BMJ. 2019;367:16317.

17. Porter ME. What is value in health care? NEnglJ Med. 2010;363(26):2477-2481. 


\section{Pursuing High-Value, Cost-Conscious Care}

18. Spencer-Bonilla G, Quinones AR, Montori VM, International Minimally Disruptive Medicine W. Assessing the Burden of Treatment. J Gen Intern Med. 2017;32(10):1141-1145.

19. Huber M, Knottnerus JA, Green L, et al. How should we define health? BMJ. 2011;343:d4163.

20. WHO. https://www.who.int/.

21. Mead N, Bower P. Patient-centredness: a conceptual framework and review of the empirical literature. Soc Sci Med. 2000;51(7):1087-1110.

22. Barry MJ, Edgman-Levitan S. Shared decision making--pinnacle of patient-centered care. $N$ EnglJ Med. 2012;366(9):780-781.

23. Laine C, Davidoff F. Patient-centered medicine. A professional evolution. JAMA. 996;275(2):152-156.

24. Baker A. Crossing the Quality Chasm: A New Health System for the 21st Century. In: Crossing the Quality Chasm: A New Health System for the 21st Century. Washington (DC)2001.

25. Montori VM. Turning Away From Industrial Health Care Toward Careful and Kind Care. Acad Med. 2019;94(6):768-770.

26. Cruess RL, Cruess SR, Boudreau JD, Snell L, Steinert Y. A schematic representation of the professional identity formation and socialization of medical students and residents: a guide for medical educators. Acad Med. 2015;90(6):718-725.

27. Education TACfGM. The Milestones Guidebook. 2016.

28. Williams. High Value Care Curriculum (HVC) 4.0: ACP; 2012 [cited 2018 01-07-2018]. 4.0: [Available from: https://www.acponline.org/clinical-information/high-value-care/medical-educatorsresources/newly-revised-curriculum-for-educators-and-residents-version-40.]

29. Berwick DM, Nolan TW, Whittington J. The triple aim: care, health, and cost. Health Aff (Millwood). 2008;27(3):759-769.

30. Weinberger SE. Providing high-value, cost-conscious care: a critical seventh general competency for physicians. Ann Intern Med. 2011;155(6):386-388.

31. Woloshin S, Schwartz LM. Overcoming overuse: the way forward is not standing still-an essay by Steven Woloshin and Lisa M Schwartz. BMJ. 2018;361:k2035.

32. Hood VL, Weinberger SE. High value, cost-conscious care: an international imperative. European Journal of Internal Medicine. 2012;23(6):495-498.

33. Brown MM BM, Sharma S. . Evidence-Based to Value-Based Medicine. Chicago: AMA Press; 2005.

34. Levinson W. Choosing Wisely Campaign. Accessed.

35. Smith CD, Alliance for Academic Internal Medicine-American College of Physicians High V, CostConscious Care Curriculum Development C. Teaching high-value, cost-conscious care to residents: the Alliance for Academic Internal Medicine-American College of Physicians Curriculum. Ann Intern Med. 2012;157(4):284-286.

36. Ajzen. Handbook of theories of social psychology (volume 1). London: SAGE publications 2012.

37. Post J, Reed D, Halvorsen AJ, Huddleston J, McDonald F. Teaching high-value, cost-conscious care: improving residents' knowledge and attitudes. Am J Med. 2013;126(9):838-842.

38. Mordang SBR, Konings KD, Leep Hunderfund AN, Paulus ATG, Smeenk F, Stassen LPS. A new instrument to measure high value, cost-conscious care attitudes among healthcare stakeholders: development of the MHAQ. BMC Health Serv Res. 2020;20(1):156.

39. Moser EM, Fazio SB, Packer CD, et al. SOAP to SOAP-V: A New Paradigm for Teaching Students High Value Care. Am J Med. 2017;130(11):1331-1336 e1332.

40. Glicken AD, Merenstein GB. Addressing the hidden curriculum: understanding educator professionalism. Med Teach. 2007;29(1):54-57. 
41. Hafferty FW, Franks R. The hidden curriculum, ethics teaching, and the structure of medical education. Acad Med. 1994;69(11):861-871.

42. Asch DA, Nicholson S, Srinivas S, Herrin J, Epstein AJ. Evaluating obstetrical residency programs using patient outcomes. JAMA. 2009;302(12):1277-1283.

43. Dornan T, Medical Education: Theory and Practice. 2011.

44. Ludmerer KM, Johns MM. Reforming graduate medical education. JAMA. 2005;294(9):1083-1087.

45. Kilminster S, Cottrell D, Grant J, Jolly B. AMEE Guide No. 27: Effective educational and clinical supervision. Med Teach. 2007;29(1):2-19. 


\section{Chapter 2}

\section{Training Physicians to Provide High-value, Cost-conscious Care: A Systematic Review}

Stammen, L.A., Stalmeijer, R. E., Paternotte, E., Pool,A. O., Driessen, E.W., Scheele, F., \& Stassen, L.P.(20 I5).Training physicians to provide high-value, cost-conscious care: a systematic review. Jama, 3 I 4(22), 2384-2400. 


\section{Abstract}

Importance: Increasing healthcare expenditures are taxing the sustainability of the healthcare system. It is important that physicians be prepared to deliver high-value, cost-conscious care.

Objective: To understand how the delivery of high-value, cost-conscious care is learned, and which circumstances influence this learning process, with a goal of informing development of effective educational interventions.

Evidence Review: PubMed, EMBASE, the Education Resources Information center (ERIC), and Cochrane databases were searched from inception until September 5, 2015. The initial search identified 2,650 articles, of which 79 met the final inclusion criteria. Data extraction was guided by a merged and modified version of a Best Evidence in Medical Education (BEME) abstraction form and a Cochrane data coding sheet. Articles were analyzed using the realist review method, a narrative review technique that focuses on understanding the underlying mechanisms in interventions. Recurrent patterns were identified in the data through thematic analyses. Resulting themes were discussed within the research team until consensus was reached.

Findings: 79 articles were included, of which 14 were randomized clinical trials. The majority of the studies were conducted in North America (78.5\%) using pre-post interventional design (58.2\%, at least 1619 participants they focused on practicing physicians $(36.7 \%, \mathrm{~N}>3448)$, resident physicians $(6.3 \%, \mathrm{~N}=516)$, medical students $(15.2 \%, \mathrm{~N}=275)$. Among the $14 \mathrm{RCTs}$, 12 addressed knowledge transmission, seven reflective practice, and one supportive environment; $10(71 \%)$ concluded that the intervention was effective. The data analysis suggested that three factors aid successful learning: 1) effective transmission of knowledge, related for example to general health economics and prices of health services, to scientific evidence regarding guidelines and the benefits and harms of healthcare, and to patient preferences and personal values (67 articles); 2) facilitation of reflective practice, such as providing feedback or asking reflective questions regarding decisions related to laboratory ordering or prescribing to give trainees insight into their past and current behavior (56 articles); and 3) creation of a supportive environment in which the organization of the healthcare system, the presence of role models of delivering high-value, cost-conscious care and a culture of high-value, cost-conscious care reinforce the desired training goals (27 articles).

Conclusions and relevance: Research on educating physicians to deliver high-value, cost-conscious care suggests that learning by practicing physicians, resident physicians, and medical students is promoted by combining specific knowledge transmission, reflective practice, and a supportive environment. These factors should be considered when educational interventions are being developed. 


\section{Pursuing High-Value, Cost-Conscious Care}

\section{Introduction}

Increasing costs of healthcare are a cause of concern to patients, governments, health economists, and the medical profession around the world. ${ }^{1-3}$ The United States has the highest healthcare expenses, with healthcare expenditure in 2015 approaching $18 \%$ of gross domestic product. ${ }^{4}$ Leading physician associations, such as the American College of Physicians, the Alliance for Academic Internal Medicine, and the American Board of Internal Medicine, offer educational programs on providing high-value, cost-conscious care.,

High-value, cost-conscious care refers to care that aims to assess the benefits, harms, and costs of interventions, and consequently to provide care that adds value. ${ }^{7}$ Although in recent years the harms of increasing healthcare costs have been acknowledged, bending the cost curve has proved difficult. ${ }^{8}$ Besides the increase in healthcare costs, and its associated complications such as the accessibility and sustainability of healthcare, quality cannot always be established. ${ }^{2,9}$ Interventions targeting physicians and their medical expertise are proposed as a means to reduce healthcare waste (care that is not beneficial to patients) while maintaining the quality of care. ${ }^{2,7}$

This review was conducted to gain understanding of how and under what circumstances educational interventions may help practicing physicians, resident physicians, and medical students deliver high-value, cost-conscious care. Insight into this learning process is necessary to develop programs that train physicians in providing such care.

\section{Methods}

This review was conducted and reported in accordance with the RAMESES publication standards for realist reviews. ${ }^{10}$ Realist review is a systematic, theory-driven, interpretative, narrative technique, which is developed to analyze heterogeneous evidence (qualitative and quantitative data and/or different outcome measures) to understand the underlying mechanisms of an intervention. Realist review gives priority to understanding what does or does not make an intervention effective over the outcome of the intervention. Included articles were analyzed by searching for information on contexts or settings in which the study was conducted, working mechanisms of the interventions, outcomes of the interventions, and program theories (i.e. theories of how and why an intervention causes effect) which could explain effectiveness of the described interventions. The realist review method has a particular strength when designs like randomized clinical trials (RCTs) have produced inconsistent estimates of efficacy and there is no consensus on when, how, and with whom to use these interventions. ${ }^{10}$ 


\section{Literature search}

The research team designed a strategy to search the PubMed, EMBASE, ERIC, and Cochrane databases. Search terms were used to identify learners (physicians, residents, medical students, medical education) and topic (cost awareness, cost consciousness, unnecessary procedures). These were combined and searched with and without factors associated with cost containment (cost effectiveness, risk assessment, value-based care, shared decision-making, practice variation, stewardship of resources). The search was conducted from the inception of each database until September 5, 2015.

\section{Study selection}

After an initial search, titles and abstracts were screened by three independent researchers (LS, AOP, IV) based on inclusion criteria: abstract available, relevant topic, implementation of intervention, evaluation of intervention, educational components in intervention, and appropriate target group. Articles without an abstract were excluded from review. When title or abstract alone did not provide enough information, full-text review was done. Articles were assessed using an eligibility form based on the BEME abstraction form and a Cochrane data coding sheet (Appendix 2.1). ${ }^{11,12}$ Articles that were found appropriate underwent further evaluation to determine if they met the final inclusion criteria (Box). If during full-text review articles did not met all five inclusion criteria, they were excluded from further review. For a realist review, both qualitative and quantitative articles can be included and analyzed to create a broad range of potential empirical mechanisms of the interventions. ${ }^{10}$

\section{Box I. Final Inclusion Criteria ${ }^{a}$}

Article is about at least one of the following topics

- Reducing volume of health care services

- Reducing health care expenditures

- Improving quality of care

- Improving knowledge and/or attitude regarding costs of care
Article describes an intervention implementation

- Intervention is evaluated

- Intervention contains educational component(s)

- Target group contains physicians, residents, and/or medical students

a To be included, articles had to meet all criteria described above.

\section{Data extraction and synthesis}

To abstract data from the selected articles in order to identify factors that may influence learning by practicing physicians, resident physicians, and medical students, three researchers used a merged and modified version of a BEME abstraction form and a Cochrane data coding sheet 


\section{Pursuing High-Value, Cost-Conscious Care}

(Appendix 2.2). ${ }^{11,12}$ Disagreements in the final phase of study selection were resolved by inviting other members of the research team to read articles in full. Data synthesis was guided by realist methodology: for each article, we identified program theories, context, mechanisms, and outcomes through a thematic analysis. ${ }^{13}$ Recurrent patterns of context and outcome in the data were identified. Next we sought to explain these patterns by their mechanism to gain understanding of how contexts, mechanisms, and outcomes are related. Both successes and failures can provide valuable information regarding context and mechanism. ${ }^{10}$ The themes were discussed until consensus was reached within the research team. Particular attention was paid to discrepant examples to ensure that the analysis could account for their occurence. Quality of the included articles was assessed based on the relevance and rigor of the articles. ${ }^{10}$ Rigor was defined as to what extent the method used to generate that particular piece of data was credible (internal validity) and trustworthy (reliability) (high or low). Relevance was determined by assessing whether the article contributed to answering the research question (high or low). Assessments of rigor and relevance were conducted by two independent researchers (LS,AOP) and were combined in one score: high (rigor high/ relevance high), medium (rigor high/ relevance low or rigor low/ relevance high) or low (rigor low/ relevance low) (Appendix 2.3). The reviewers were not blinded to any portion of articles. Disagreement about study selection, data extraction, and data synthesis were resolved through consensus within the research team.

\section{Results}

\section{Search results and selected articles}

The results of the review process are summarized in Appendix 2.4. A total of 2,650 articles were considered for review; 102 articles were appropriate for further selection, of which 79 met the inclusion criteria. The majority of the studies were conducted in North America (78.5\%) using pre-post interventional design (58.2\%, N>1619 participants); they focused on practicing physicians (36.7\%, $\mathrm{N}>3448)$, residents $(6.3 \%, \mathrm{~N}=516)$, medical students $(15.2 \%, \mathrm{~N}=275)$. 28 articles did not report the number of participating practicing physicians, resident physicians, or medical students. More detail regarding the characteristics of the selected articles are shown in Table 3. Of the articles, $87 \%$ concluded that their interventions were effective in delivering appropriate care, reducing costs, volume or unnecessary procedures. Among the 14 RCTs, 12 addressed knowledge transmission, seven reflective practice, and one supportive environment; ten $(71 \%)$ concluded that the intervention was effective. Summaries of articles are provided in Table 4, with more details provided in Appendix 2.3. 
Table 3: Characteristics of included studiesa

\begin{tabular}{|c|c|c|c|}
\hline & & Frequency & Percentage \\
\hline \multirow[t]{5}{*}{ Geographical Setting } & North America & 62 & $78.5 \%$ \\
\hline & Asia & 6 & $7.6 \%$ \\
\hline & Europe & 6 & $7.6 \%$ \\
\hline & Oceania & 4 & $5.0 \%$ \\
\hline & Africa & 1 & $1.3 \%$ \\
\hline \multirow[t]{5}{*}{ Study Designs } & Pre-post interventional $(\mathrm{N}>1619)$ & 46 & $58.2 \%$ \\
\hline & Quasi-experimental $(N>616)$ & 14 & $17.7 \%$ \\
\hline & Randomized Controlled Trial $(\mathrm{N}>2149)$ & 14 & $17.7 \%$ \\
\hline & Mixed methods $(\mathrm{N}>349)$ & 4 & $5.1 \%$ \\
\hline & Qualitative (N=3I) & 1 & $1.3 \%$ \\
\hline \multirow[t]{5}{*}{ Target group } & Practicing physicians & 29 & $36.7 \%$ \\
\hline & Physicians \& Healthcare Professionals & 18 & $22.6 \%$ \\
\hline & Combination of physicians, residents and students & 15 & $19.0 \%$ \\
\hline & Resident physicians & 12 & $15.2 \%$ \\
\hline & Medical students & 5 & $6.3 \%$ \\
\hline \multirow[t]{5}{*}{ Number of Participants } & Not reported & 28 & $35.4 \%$ \\
\hline & $0-50$ & 25 & $31.7 \%$ \\
\hline & $51-100$ & 14 & $17.7 \%$ \\
\hline & $101-150$ & I & $1.3 \%$ \\
\hline & $>150$ & 11 & $13.9 \%$ \\
\hline \multicolumn{4}{|l|}{ Training Factors } \\
\hline \multicolumn{2}{|c|}{ Knowledge transmission, reflective practice and supportive environmentb } & 16 & $20.2 \%$ \\
\hline \multicolumn{2}{|c|}{ Knowledge transmission and reflective practice ${ }^{c}$} & 29 & $36.7 \%$ \\
\hline \multicolumn{2}{|c|}{ Knowledge transmission and supportive environment $\mathrm{t}^{35,36,44,50,51}$} & 4 & $5.1 \%$ \\
\hline \multicolumn{2}{|c|}{ Reflective practice and supportive environment ${ }^{39,7,4,77,78,91,92}$} & 6 & $7.6 \%$ \\
\hline \multicolumn{2}{|l|}{ Knowledge transmission ${ }^{d}$} & 18 & $22.8 \%$ \\
\hline \multicolumn{2}{|c|}{ Reflective practice ${ }^{21,40,66,67,73}$} & 5 & $6.3 \%$ \\
\hline \multicolumn{2}{|l|}{ Supportive environment ${ }^{87}$} & 1 & $1.3 \%$ \\
\hline
\end{tabular}

a Study designs and target groups are based on Best Evidence Medical Education Collaboration classification. Types of training factors in each study are counted based on which factor they represent in the Results section of the text. Twenty-eight included articles did not report the number of participants. Therefore, the minimum number of participants based on those who could be counted is reported.

${ }^{\mathrm{b}}$ References $27,32,37,41,46,55,61,64,65,69,70,71,81-83$, and 88 .

${ }^{c}$ References 14-16, 22, 28-31, 34, 42-45, 47-49, 52, 53, 56, 57, 62, 63, 68, 72, 75, 76, 79, 80, 84, and 86.

${ }^{\mathrm{d}}$ References 17-20, 23-26, 33, 38, 54, 58-60, 85, 89, and 90 .

The data analysis of the included articles concluded that the success of educational interventions preparing future and practicing physicians for the delivery of high-value, cost-conscious care may depend on three factors: knowledge transmission, reflective practice, and a supportive environment. These three factors were the result of an analytic process in which we first collected potential program theories, contexts, mechanisms, and outcomes as presented in the included 


\section{Pursuing High-Value, Cost-Conscious Care}

articles. Second, we identified recurrent patterns of contexts and outcomes that could be explained by mechanisms found in the included articles. These patterns were extensively discussed within the entire research team in order to identify overarching factors that influence the learning of practicing physicians, resident physicians, and medical students.

\section{Knowledge transmission}

Increasing knowledge about high-value, cost-conscious care behavior among practicing physicians, resident physicians, and medical students has been associated with reduction in unnecessary or inappropriate healthcare delivery. Such knowledge interventions specifically focused on three subject areas: prices of services and general health economics, scientific evidence, and patient preferences and were represented in 67 articles (87\%).

\section{Prices and general health economics}

The majority of interventions focused on raising awareness of prices of medical services and on teaching the basics of health economics. ${ }^{14-30}$ The first was done by presenting prices on modified order forms, on pocket cards, or in web-based tools, or by visualizing price indicators. ${ }^{14,16-18,21-24,27}$ A high-quality study by Ellemdin et a ${ }^{18}$ used a pocket-sized brochure to inform internal medicine physicians $(\mathrm{N}=434)$ about the costs of laboratory testing and asked them to write these costs on the order form. Over the 4-month follow-up period there was a $27-36 \%$ decrease in laboratory expenditures. Teaching physicians the basics of general health economics ${ }^{15,19,20,25,26,28}$ was done by lectures and interactive sessions informing physicians about the competitive market forces and general healthcare economic principles, such as the role of insurance companies in price setting. ${ }^{19,26}$ Although the weekly lectures for medical interns were associated with a nonsignificant reduction in the number of tests ordered during patient admissions ${ }^{19}$, the interactive sessions significantly improved physicians' knowledge of economics and rational prescribing. ${ }^{26}$

\section{Scientific evidence}

A frequent approach to teach efficiency was to provide the background evidence underlying indications and guidelines related to medical decision-making. ${ }^{14,15,19,21,24-26,31-63}$ Before implementing or discussing new ${ }^{26,32,42,47,53,55,62}$, modified, ${ }^{44,50,64}$ or established guidelines $^{32-34,39,41,43,49,54}$ or clinical indications ${ }^{28,48,56,65}$, trainees and physicians were informed of the scientific evidence that supported these recommendations. A high-quality study by Lee et $\mathrm{al}^{61}$ implemented a teaching session focusing on guidelines on antibiotic prescriptions among 42 practicing physicians, resident physicians, and medical students. These teaching sessions were accompanied by a checklist addressing dose, route, duration, and appropriateness of prescriptions. Their intervention resulted in an annual saving of $\$ 69.424$ (baseline $\$ 149.743$ versus $\$ 80.319$ post-intervention). Effects associated with such teaching depended on several 
factors, such as expert involvement, complementary lectures, and timing of information. For example, when guidelines were developed by expert panels, they were accepted and adhered to more readily, especially when constructed in multidisciplinary teams. ${ }^{32,40,44}$ Additionally, several interventions had an expert deliver didactic sessions or conduct outreach visits on the clinical topic to which the guideline applied. ${ }^{32,34,35,46,47,54,66}$ Other interventions introduced guidelines using lectures about the key recommendations ${ }^{42,55}$ or by email notifications. ${ }^{25}$

Another technique that was often used with the aim of increasing the likelihood that physicians would implement the guidelines was to provide just-in-time scientific evidence. For example, inserting guidelines or recommendations into charts allowed physicians to successfully translate these guidelines immediately into practice. ${ }^{24,33,45,47,54}$ Complementing guidelines by suggestions to physicians for treatment of selected patients formed the core of several interventions aimed to improve physicians' prescribing patterns. ${ }^{33,37,45,49,51}$ Two of these interventions used forms that were inserted in charts of selected patients, which were associated with a significant reduction in the prescription of the targeted pharmaceuticals. ${ }^{33,37}$ For example, the change from a secondgeneration cephalosporin to a less expensive but equally effective first-generation cephalosporin occurred in $28 \%$ of cases (medium-quality study). ${ }^{33}$ Moreover, when physicians were able to immediately accept or decline a suggestion to switch to a different drug, and to modify their prescription to the more appropriate evidence-informed suggestion, a prescribing reduction up to $43 \%$ was achieved (medium-quality study). ${ }^{37}$

\section{Patient preferences}

Four studies were identified that sought to stimulate high-value, cost-conscious care by improving understanding of patient preferences. ${ }^{24,39,57,58}$ Physicians involved patients in the decision-making process and in devising a plan of action. During consultation, physicians discussed risks and benefits of medical services and explored patient concerns in order to be able to provide the best possible care. ${ }^{24,57}$ Knowledge of patients' preferences was deemed sufficient when patients felt comfortable about the shared decision that was made. Educational interventions such as DECISION+ combined evidence and patient preferences in workshops to optimize the prescription of antibiotics for acute respiratory infections by residents and practicing physicians $s^{57,58}$; the number of patients opting for immediate antibiotic therapy in this high-quality study declined $16 \%(49 \%[\mathrm{~N}=70]$ control group vs $33 \%$ [N=81] experimental group), but this was not statistically significant $(\mathrm{P}=0.08) .{ }^{57}$

\section{Reflective practice}

A second derived factor was to stimulate reflective practice in future and practicing physicians to help them gain insight into their performance, with a goal of influencing future behavior. ${ }^{16,21,22,25,31,29,34,35,37,39,40,49,52,61,65,67-74}$ This was achieved by providing feedback, 


\section{Pursuing High-Value, Cost-Conscious Care}

stimulating reflection, or a combination of both, in 56 articles including more than 2039 participants.

\section{Feedback}

Feedback to physicians about their performance was most often based on utilization data such as the number of ordered tests or amount of prescribed antibiotics. ${ }^{14,21,22,28-30,34,37,43,49,52,55,61,65,67-}$ 70,75-77 This feedback could focus on various elements which, in turn, influenced how trainees learned from feedback. Feedback could focus on volume (such as numbers of requested tests) 43,48,55,75-77, costs (eg, total costs of antibiotic prescriptions), ${ }^{14,21,22,28}$ or appropriateness (eg, extent of adherence to guidelines). ${ }^{67}$ Feedback could center on physicians' individual utilization or their utilization in relation to that of others. ${ }^{34,49,52,67,76}$ Another element of importance was the frequency of feedback. ${ }^{21,40,67}$ One high-quality study sent emails to 15 physicians in the upper 50 th percentile for expenditure to point out the differences in their spending behavior compared to their peers over a 3 -month period. ${ }^{67}$ This measure did not lead to a significant expenditure reduction compared to the control group (per physician expenditure difference of $\$ 654.45$; $\mathrm{p}=0.64$ ), which might have been due to the short amount of time between feedback provision and measurement of changes in spending behavior. In contrast, Stuebing and $\mathrm{Miner}^{21}$ made use of more frequent feedback: in their medium-quality study, attending physicians and residents in a surgical department (number not reported) received weekly notifications about the costs, expressed in terms of dollars per ICU patient per day. Despite small increases in total expenditure that occurred each time residents switched services, there was a significant decreasing trend in total laboratory costs. ${ }^{21}$ At baseline, laboratory expenditures were $\$ 147.73$ per patient per day, and a decrease of up to $27 \%$ (-\$39.62) from baseline in expenditure per day was reached.

\section{Reflective questions}

Peer- or supervisor-facilitated reflection on medical decisions and their influence on the quality and cost effectiveness of care was the focus of several studies. ${ }^{15,16,22,25,27,34,35,39,40,53,55,56,64,66,69,71-74,76,78}$ Supervisors ${ }^{39,55,72}$, peers ${ }^{69,79}$, and experts ${ }^{27,37,53,56,64,66,76}$ in several clinical settings were the initiators of reflective discussions. ${ }^{22,25,34,39}$ For example, they asked reflective questions during morning rounds or grand rounds and in the presence of other healthcare professionals. Reflective practice was guided by questions such as "what was the indication for this test?", "what alternatives were available?", or "why do you think this specific test is redundant?". ${ }^{16,39}$ Reflection on one's own behavior was further stimulated by means of reminder alerts whenever a laboratory request or prescription was ordered that was identified as redundant. ${ }^{73,80}$ Although determining what classified as redundancy from the perspective of the research team and the participants was difficult, in a medium-quality study ${ }^{73}$ the alerts appeared to be effective and resulted in cancellation of $69 \%$ of redundant tests by physicians $(\mathrm{N}=282)$ compared to $51 \%$ in the control group $(\mathrm{N}=4769)(\mathrm{P}<0.001)$. 


\section{Combining feedback and reflection}

Eight articles used feedback as a starting point for reflection. ${ }^{31,34,43,49,68,69,77,81}$ The authors chose this combination to counter the potential risk of creating a judgmental setting, which is not conducive to high-value, cost-conscious behavior. ${ }^{49}$ Von Ferber et al ${ }^{69}$ introduced peer-to-peer review discussion groups (high-quality study). During these sessions the motives that influence prescribing behavior and attitudes of physicians $(\mathrm{N}=79)$, together with underlying causes of practice variation, were discussed in an open and tolerant environment. This approach was associated with significant decreases in prescription costs from $\$ 853$ to $\$ 527$ for high-prescribers, and from $\$ 469$ to $\$ 352$ for low-prescribers $\left(\mathrm{P}<.001\right.$ for both), and with cost-effective prescription of selected drugs. ${ }^{69}$

\section{Supportive learning environment}

The data from 27 articles including more than 521 participants highlighted the essential role of the environment in which educational interventions are implemented. Support at the macro level, such as supportive payment systems, management policies, and reimbursement systems, was emphasized, together with the presence of clinical role models and teachers, and a culture of high-value, cost-conscious care.

\section{Macro level support}

There was a repeated emphasis in several publications $(\mathrm{N}=29,37 \%)$ on the association of macro level support for their interventions on practicing physicians, resident physicians, and medical students. ${ }^{16,17,19,22,24,25,28,31-33,44,47,48,50,55,66,68,77,82,83}$ The organization was often perceived to frustrate the training of physicians in the provision of high-value, cost-conscious care. ${ }^{36,44,57,83-86}$ This was the case, for example, when physicians felt inhibited by fear of malpractice suits ${ }^{84,85}$ and pressured to adhere to guidelines. ${ }^{57}$ Other elements of the healthcare system that might either positively or negatively influence the delivery of high-value, cost-conscious care were the availability of resources $^{15,31,34,87}$, workload ${ }^{36,66,84}$, bureaucratic structure ${ }^{55,84}$, and access to and transparency of healthcare costs. ${ }^{16,25}$ For example, in a high-quality survey study by Post et al. ${ }^{16}$ of 68 residents with an $83 \%$ response rate, only four claimed that they had adequate access to the costs of care they provided. Furthermore, 59 of these residents claimed that better knowledge of costs would influence their ordering behavior. The use of incentives (financial or otherwise) to modify practice $^{28,47,55,76,84,86}$ and payment systems $s^{17,19,22,28,66,76,77,82,86}$ are also examples of how macro level decisions could influence individual physicians. The influence of the reimbursement system on the success of cost-containment interventions was identified by Lyle et $\mathrm{a}^{86}$. In this high-quality study, it was noted that the structure for financing hospital care could run counter to hospital economics, since a decrease in utilization is related to a decline in third-party payments. ${ }^{86}$

\section{Clinical role models and teachers}

An environment in which learners have role models who are very much committed to delivering and demonstrating high-value, cost-conscious care was often mentioned as an important factor in preparing physicians for sustainable practice. ${ }^{15,19,20,22,34,35,40,47,49,66,79,84}$ The lack of such clinical role 


\section{Pursuing High-Value, Cost-Conscious Care}

models was described as the most common barrier to lessons learned. ${ }^{22,40,48,84}$ Some authors noted that residents seeking to learn how to provide high-value, cost-conscious care received little or no support from supervisors or attending physicians. For example, the study by Post et $\mathrm{al}^{16} \mathrm{implemented}$ an intervention among 83 internal medicine residents based on knowledge of costs and reflective discussions regarding the appropriateness of health care services for selected patients. Their evaluation included a survey $(\mathrm{N}=68)$; only 26 residents agreed with the statement 'my supervising consultants consistently encourage me to consider costs when making medical decisions'. As a result, residents became disheartened by fear of getting into conflict with supervisors or being overruled by them. ${ }^{16,22,40}$

The importance of the involvement of local role models and respected teachers in educational programs was acknowledged by several studies. ${ }^{19,20,26,28,34,35,47,49,50,57,66,69,76,77,85}$ To enhance acceptance of educational interventions, people with established clinical expertise, managerial responsibilities, or physicians considered as opinion leaders were given a prominent place in the program. ${ }^{19,47,49,50,57,66,69,76,81,85}$ Illustrating the influence of teachers on training effectiveness, one study asserted that if workshops were not conducted by a highly motivated principal teacher, the effect of the intervention could not be reproduced. ${ }^{57}$

\section{The culture of interprofessional collaboration in relation to high-value, cost-conscious} care

Learning within interprofessional collaborations compared with individual training of physicians was the focus of 14 interventions. ${ }^{37,44-46,48,51,55,61,65,66,68,70,74,79,83,88,89}$ Associations with learning in collaborations was demonstrated by a medium-quality study by Collins et al, ${ }^{89}$ who sent participants identical feedback emails discussing the appropriateness of dipyramidole prescriptions. A physicians-only control group $(\mathrm{N}=342)$ compared with a physicians-pharmacists intervention group $(\mathrm{N}=91)$ spent $\$ 37.01$ per patient more on dipyridamole $(\mathrm{p}<0.025)$, even though specific pharmacist action had not been solicited.

The importance of the values and beliefs of coworkers, whether peers or other health professionals, was highlighted by several other studies. ${ }^{22,25,27,32,33,36,37,39-41,46,47,50,51,55,57,64,77,84,88,90-92}$ By raising cost issues in newsletters, ${ }^{27,32,46,50,51,88,90}$ in posters, ${ }^{32,33,36,37,50,57,64,91}$ in grand rounds, or at bedside teaching ${ }^{25,40,50,91}$, these studies aimed to create a pro-sustainability environment. ${ }^{22,36,37,55,88}$ In a high-quality study ${ }^{55}$, feedback of participating physicians after an intervention that aimed to reduce the costs of laboratory ordering described that group discussions among staff increased consensus regarding the general understanding for the need of cost reduction in the emergency department. Additionally, the involvement of the nursing staff was defined by the authors as one of the key success factors of the intervention, due to active involvement of the nursing staff as a moderator in the discussions regarding appropriateness between residents and attending physicians. Declaring cost effectiveness as the theme of the week was another tactic used to stimulate cost consciousness. ${ }^{41}$ Three articles discussed the negative effect of the absence of an interprofessional collaborating culture toward high-value, cost-conscious care. ${ }^{39,84,92}$ 
Table 4: Overview of included articles NR= not reported.

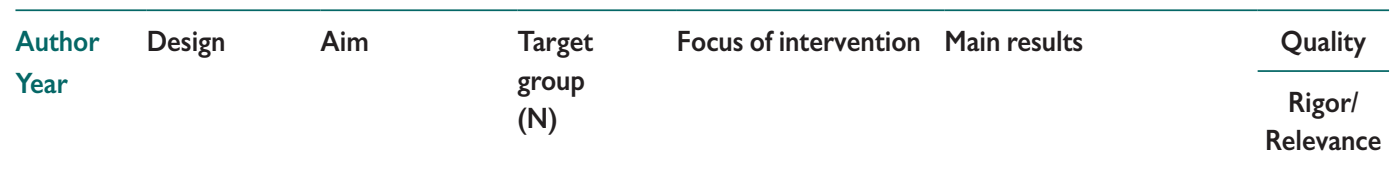

Articles that addressed all three factors (knowledge transmission, reflective practice, supportive environment)

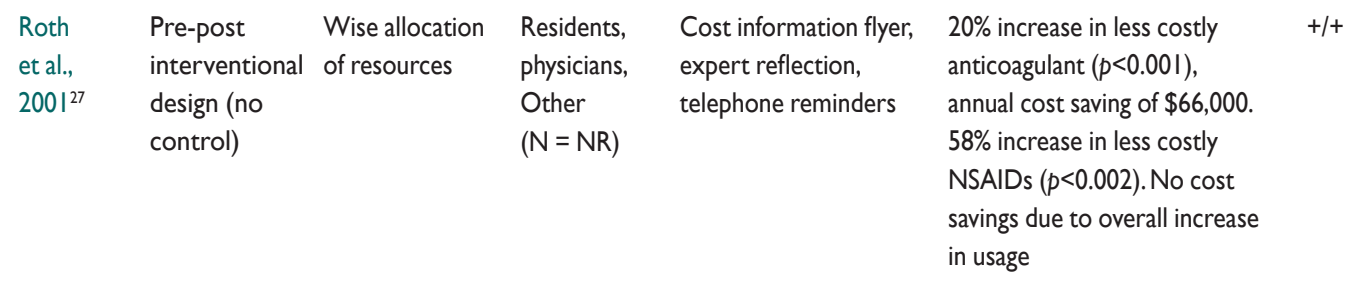

de Leon Pre-post Decrease in rate Residents, Guidelines and Significant reduction of -/-

et al., interventional of inappropriate physicians, prescribing-indications, inappropriate stress ulcer

$2013^{32}$ study use of drugs other educational sessions, EB prophylaxis ( $19 \%$ vs $6.6 \%$,

$(N=N R) \quad$ facilitator guide for $\quad p=0.02)$

discussion

\begin{tabular}{|c|c|c|c|c|c|}
\hline $\begin{array}{l}\text { Barbarello } \\
\text { et al., } \\
2006^{37}\end{array}$ & $\begin{array}{l}\text { Pre-post } \\
\text { interventional } \\
\text { study }\end{array}$ & $\begin{array}{l}\text { Increase } \\
\text { efficiency } \\
\text { Increase cost } \\
\text { effectiveness }\end{array}$ & $\begin{array}{l}\text { Physicians, } \\
\text { other } \\
(N=N R)\end{array}$ & $\begin{array}{l}\text { Educational } \\
\text { communication forms } \\
\text { and posters, patient- } \\
\text { specific physician- } \\
\text { pharmacists discussion }\end{array}$ & $\begin{array}{l}\text { Decrease of monthly } \\
\text { expenditure in three out of } \\
\text { four target medications }\end{array}$ \\
\hline
\end{tabular}

Dowling Pre-post Decrease in not Residents Feedback, EBM Significant reduction in TSH $\quad+/+$

et al., interventional indicated testing $(N=20)$

$1989^{41}$ study

indications, cost

effectiveness theme for

preclinical talks test ordering $(p<0.000 \mathrm{I})$ and

CBCs $(p=0.05)$. Percentage

of appropriate TSH tests indicated increased $(p<$ 0.001 )

Sucov Pre-post Decrease in tests Residents, et al., interventional that are not other

$1999^{55}$ study, time clinically $\quad(N=N R)$ series. indicated patients $(p<0.00 \mathrm{I}) . \$ 50.000$ 100.000 less charged
Guideline, lecture on

EBM and cost

containment, discuss

ordering with

attending physician,

utilization feedback
Significant decline in total 
Table 4: Overview of included articles (continued) NR= not reported.

\begin{tabular}{|c|c|c|c|c|c|c|}
\hline \multirow{2}{*}{$\begin{array}{l}\text { Author } \\
\text { Year }\end{array}$} & \multirow[t]{2}{*}{ Design } & \multirow[t]{2}{*}{ Aim } & \multirow{2}{*}{$\begin{array}{l}\text { Target } \\
\text { group } \\
(\mathrm{N})\end{array}$} & \multirow[t]{2}{*}{ Focus of intervention } & \multirow[t]{2}{*}{ Main results } & Quality \\
\hline & & & & & & $\begin{array}{c}\text { Rigor/ } \\
\text { Relevance }\end{array}$ \\
\hline $\begin{array}{l}\text { Lee } \\
\text { et al., } \\
2014^{61}\end{array}$ & $\begin{array}{l}\text { Pre-post } \\
\text { interventional } \\
\text { study, time } \\
\text { series }\end{array}$ & $\begin{array}{l}\text { Decrease in } \\
\text { unnecessary } \\
\text { antibiotic use }\end{array}$ & $\begin{array}{l}\text { Physicians, } \\
\text { residents, } \\
\text { medical } \\
\text { students } \\
(\mathrm{N}=42)\end{array}$ & $\begin{array}{l}\text { Teaching session } \\
\text { regarding importance } \\
\text { of } A B \text { stewardship, } \\
\text { guidelines and } \\
\text { recommendations. } \\
\text { Implementation of } \\
\text { checklist for time-out } \\
\text { audit and feedback } \\
\text { regarding } \\
\text { appropriateness }\end{array}$ & $\begin{array}{l}\text { I5\% of time-out audit led to } \\
A B \text {-change.Annual costs of } \\
A B \text { decreased }(\$ 149743 \text { to } \\
\$ 803 \mid 9) \text {. Volume of } A B \\
\text { unchanged. No significant } \\
\text { changes in quality outcomes }\end{array}$ & $+/+$ \\
\hline
\end{tabular}

\begin{tabular}{|c|c|c|c|c|c|c|}
\hline $\begin{array}{l}\text { Wein } \\
\text { et al., } \\
1987^{64}\end{array}$ & $\begin{array}{l}\text { Pre-post } \\
\text { interventional } \\
\text { design (no } \\
\text { control } \\
\text { group) }\end{array}$ & $\begin{array}{l}\text { Improve cost- } \\
\text { effective utilization }\end{array}$ & $\begin{array}{l}\text { Medical staff, } \\
\text { other } \\
(N=N R)\end{array}$ & $\begin{array}{l}\text { Utilization criteria, } \\
\text { newsletter, expert } \\
\text { reflection on } \\
\text { prescribing }\end{array}$ & $\begin{array}{l}\text { Average monthly prescribing } \\
\text { costs decreased } \$ 6,300 \\
(-13.5 \%)\end{array}$ & $-/+$ \\
\hline $\begin{array}{l}\text { Gregory } \\
\text { et al., } \\
199965\end{array}$ & $\begin{array}{l}\text { Pre-post } \\
\text { interventional } \\
\text { study }\end{array}$ & $\begin{array}{l}\text { Decrease in rate } \\
\text { of cesarean } \\
\text { sections without } \\
\text { lowering health } \\
\text { care quality }\end{array}$ & $\begin{array}{l}\text { Residents, } \\
\text { physicians, } \\
\text { other ( } N= \\
\text { NR) }\end{array}$ & $\begin{array}{l}\text { Discussion indications, } \\
\text { utilization feedback, } \\
\text { focus groups on } \\
\text { attitudes regarding } \\
\text { VBAC }\end{array}$ & $\begin{array}{l}\text { C-section rate decreased } \\
\text { (21.2\%). Post-intervention } \\
\text { C-section rate increased. No } \\
\text { statistically or clinically } \\
\text { significant increase in various } \\
\text { complications }\end{array}$ & $-/-$ \\
\hline $\begin{array}{l}\text { Von } \\
\text { Ferber } \\
\text { et al., } \\
199969\end{array}$ & $\begin{array}{l}\text { Pre-post } \\
\text { interventional } \\
\text { design, time } \\
\text { series }\end{array}$ & $\begin{array}{l}\text { Improve quality } \\
\text { of practice } \\
\text { lowering costs }\end{array}$ & $\begin{array}{l}\text { Physicians } \\
(\mathrm{N}=79)\end{array}$ & $\begin{array}{l}\text { Feedback and reflection } \\
\text { with peers (Balint } \\
\text { setting), EB guideline }\end{array}$ & $\begin{array}{l}\text { Significant decrease in } \\
\text { prescription rate }(7.2-4.8) \\
\text { and costs }(\$ 853-\$ 527) \text { of } \\
\text { most targeted drugs }(p<0.05)\end{array}$ & $+/+$ \\
\hline $\begin{array}{l}\text { Moriates } \\
\text { et al., } \\
2013^{71}\end{array}$ & $\begin{array}{l}\text { Pre-post } \\
\text { interventional } \\
\text { study }\end{array}$ & $\begin{array}{l}\text { Increase in cost } \\
\text { awareness } \\
\text { Promote } \\
\text { attitude toward } \\
\text { cost-control } \\
\text { cultivating } \\
\text { cost-effective } \\
\text { behavior }\end{array}$ & $\begin{array}{l}\text { Medical } \\
\text { students, } \\
\text { residents } \\
(\mathrm{N}=55) \\
\text { physicians } \\
(\mathrm{N}=19)\end{array}$ & $\begin{array}{l}\text { Cost-awareness session, } \\
\text { price information, } \\
\text { review of EB guideline, } \\
\text { reflection on ordering, } \\
\text { stimulate EB care and } \\
\text { cost-effective care }\end{array}$ & $\begin{array}{l}\text { Positive Likert scale scores } \\
(\text { ( -5) on several items } \\
\text { 'relevant to medical practice' } \\
4.6 \text { (SD 0.6) 'likely to change } \\
\text { practice' } 4.3 \\
\text { (SD 0.7) }\end{array}$ & $+/+$ \\
\hline
\end{tabular}


Table 4: Overview of included articles (continued) NR= not reported.

\begin{tabular}{llll}
\hline Author Design & Aim & $\begin{array}{l}\text { Target } \\
\text { group }\end{array}$ & Focus of intervention Main results \\
Year & & (N)
\end{tabular}

(N)

\begin{tabular}{|c|c|c|c|c|c|c|}
\hline $\begin{array}{l}\text { Bornard } \\
\text { et al., } \\
\left.201\right|^{81}\end{array}$ & $\begin{array}{l}\text { Pre-post } \\
\text { interventional } \\
\text { study }\end{array}$ & $\begin{array}{l}\text { Increase quality } \\
\text { of prescribing by } \\
\text { decreasing } \\
\text { unnecessary } \\
\text { prescribing }\end{array}$ & $\begin{array}{l}\text { Residents } \\
(\mathrm{N}=4) \\
\text { physicians } \\
(\mathrm{N}=4)\end{array}$ & $\begin{array}{l}\text { Feedback and experts' } \\
\text { advice to improve, } \\
\text { sessions on medical } \\
\text { topics, multidisciplinary } \\
\text { discussions }\end{array}$ & $\begin{array}{l}\text { Prevalence of adequate } \\
A B \text {-prescriptions before and } \\
\text { after intervention ( } 73 \% \text { vs. } \\
80 \%, p=0.31 \text { ). More frequent } \\
\text { re-assessment of diagnosis } \\
\text { between day } 2 \text { and day } 4 \text { ( } 11 \% \\
\text { vs. } 32 \%, p=0.02) \text {, Improved } \\
\text { adaptation of } A B \text {-therapies to } \\
\text { positive microbiology }(25 \% \\
\text { before vs. } 50 \% \text { after, } p=0.18 \text { ) }\end{array}$ & $+/-$ \\
\hline $\begin{array}{l}\text { Manheim } \\
\text { et al., } \\
1990^{82}\end{array}$ & $\begin{array}{l}\text { Pre-post } \\
\text { interventional } \\
\text { study }\end{array}$ & $\begin{array}{l}\text { Increase in cost } \\
\text { awareness } \\
\text { Decrease in } \\
\text { costs }\end{array}$ & $\begin{array}{l}\text { Residents } \\
(\mathrm{N}=105)\end{array}$ & $\begin{array}{l}\text { Chart audits of high } \\
\text { costs, comparative } \\
\text { feedback on generated } \\
\text { costs, evidence } \\
\text { supported discussions } \\
\text { about cost effectiveness } \\
\text { of care }\end{array}$ & $\begin{array}{l}\text { Experimental group interns } \\
\text { had significantly lower } \\
\text { charges }(p=0.036) \text { and LOS } \\
(p=0.008) \text { per patient } \\
\text { compared to control group }\end{array}$ & $+/+$ \\
\hline $\begin{array}{l}\text { Niquille } \\
\text { et al., } \\
2010^{83}\end{array}$ & $\begin{array}{l}\text { Pre-post } \\
\text { interventional } \\
\text { study }\end{array}$ & $\begin{array}{l}\text { Containing } \\
\text { prescribing costs }\end{array}$ & $\begin{array}{l}\text { Physicians, } \\
(\mathrm{N}=18-60) \\
\text { other }\end{array}$ & $\begin{array}{l}\text { Discussion with expert } \\
\text { on (over)use, risk/ } \\
\text { benefit }\end{array}$ & $\begin{array}{l}\text { Annual drug cost per patient } \\
\text { increased } 74 \% \text { in control } \\
\text { group versus } 32 \% \text { in } \\
\text { intervention group (period of } \\
8 \text { years). Cost savings of } \\
\$ 225,000 \text { per GP }\end{array}$ & $+/-$ \\
\hline $\begin{array}{l}\text { Mallows } \\
\text { et al., } \\
2013^{88}\end{array}$ & $\begin{array}{l}\text { Pre-post } \\
\text { interventional } \\
\text { study }\end{array}$ & $\begin{array}{l}\text { Decrease in } \\
\text { ordering of tests }\end{array}$ & $\begin{array}{l}\text { Medical } \\
\text { students, } \\
\text { residents, } \\
\text { physicians } \\
(\mathrm{N}=\mathrm{NR})\end{array}$ & $\begin{array}{l}\text { Utility discussion, } \\
\text { information on costs } \\
\text { and quality of testing, } \\
\text { fine for ordering }\end{array}$ & $\begin{array}{l}\text { Significant absolute reduction } \\
\text { in rate of CRP test ordering } \\
(17.6 \% \\
p<0.001)\end{array}$ & $-/+$ \\
\hline $\begin{array}{l}\text { ljo } \\
\text { et al., } \\
2011^{46}\end{array}$ & $\begin{array}{l}\text { Quasi- } \\
\text { experimental }\end{array}$ & $\begin{array}{l}\text { Increase the } \\
\text { quality of care } \\
\text { lowering costs } \\
\text { by improving } \\
\text { prescribing }\end{array}$ & $\begin{array}{l}\text { Physicians, } \\
\text { other } \\
(\mathrm{N}=\mathrm{NR})\end{array}$ & $\begin{array}{l}\text { Feedback, peer } \\
\text { discussions, Expert } \\
\text { reflection, EB } \\
\text { newsletters }\end{array}$ & $\begin{array}{l}\text { Shorter length of stay in ICU } \\
\text { ( } 6 \text { days vs. II-36 days), } \\
\text { increase in drug cost } \\
\text { expenses ( } \$ 192 \text { with } \\
\text { infectious diseases related } \\
\text { interventions) }\end{array}$ & $-/-$ \\
\hline
\end{tabular}


Table 4: Overview of included articles (continued) NR= not reported.

\begin{tabular}{|c|c|c|c|c|c|c|}
\hline \multirow{2}{*}{$\begin{array}{l}\text { Author } \\
\text { Year }\end{array}$} & \multirow[t]{2}{*}{ Design } & \multirow[t]{2}{*}{ Aim } & \multirow{2}{*}{$\begin{array}{l}\text { Target } \\
\text { group } \\
(\mathrm{N})\end{array}$} & \multirow[t]{2}{*}{ Focus of intervention } & \multirow[t]{2}{*}{ Main results } & Quality \\
\hline & & & & & & $\begin{array}{c}\text { Rigorl } \\
\text { Relevance }\end{array}$ \\
\hline $\begin{array}{l}\text { Miyakis } \\
\text { et al., } \\
2006^{70}\end{array}$ & $\begin{array}{l}\text { Quasi- } \\
\text { experimental } \\
\text { design }\end{array}$ & $\begin{array}{l}\text { Improve quality } \\
\text { Lower costs of } \\
\text { testing }\end{array}$ & $\begin{array}{l}\text { Residents, } \\
\text { physicians, } \\
\text { other } \\
(\mathrm{N}=\mathrm{NR})\end{array}$ & $\begin{array}{l}\text { Feedback on knowledge } \\
\text { and appropriateness, } \\
\text { discussion on strategies } \\
\text { to reduce testing }\end{array}$ & $\begin{array}{l}\text { Pre-intervention } 24482 \\
\text { (I } 28.6 \%, \text { II } 69,3 \% \\
\text { avoidable) tests were } \\
\text { ordered, post- } \\
\text { intervention } 10297 \\
\text { (I } 26.7 \%, \text { II } 63,2 \%) \\
\text { avoidable) test were } \\
\text { ordered }\end{array}$ & $+/+$ \\
\hline
\end{tabular}

Articles that addressed 2 factors

\begin{tabular}{|c|c|c|c|c|c|c|}
\hline $\begin{array}{l}\text { Verstappen } \\
\text { et al., } \\
2003^{43}\end{array}$ & $\mathrm{RCT}$ & $\begin{array}{l}\text { Effective test } \\
\text { ordering }\end{array}$ & $\begin{array}{l}\text { Physicians } \\
(\mathrm{N}=174)\end{array}$ & $\begin{array}{l}\text { Reflection on feedback, } \\
\text { guidelines }\end{array}$ & $\begin{array}{l}\text { Decrease in total number of } \\
\text { tests ordered }(-67 \text { tests per } \\
\text { physician } p=0.01) \text { and } \\
\text { inappropriate tests ordered } \\
(-16 \text { test per physician } \\
p=0.01)\end{array}$ & $+/+$ \\
\hline $\begin{array}{l}\text { Bernal- } \\
\text { Delgrado } \\
\text { et al., } \\
2002^{47}\end{array}$ & $\mathrm{RCT}$ & $\begin{array}{l}\text { Change } \\
\text { prescribing } \\
\text { behavior to } \\
\text { improve medical } \\
\text { practice }\end{array}$ & $\begin{array}{l}\text { Physicians, } \\
\text { other } \\
(\mathrm{N}=\mid 10)\end{array}$ & $\begin{array}{l}\text { Outreach visit with/ } \\
\text { without EB brochure }\end{array}$ & $\begin{array}{l}\text { Experimental group } \\
\text { reduced prescriptions by } \\
22.5 \% \text {.Average cost per } \\
\text { prescription decreased by } \\
1.91 \% \text {. No statistically } \\
\text { significant difference } \\
\text { found between } \\
\text { experimental and control } \\
\text { groups }\end{array}$ & $+/-$ \\
\hline $\begin{array}{l}\text { Hux } \\
\text { et al., } \\
1999^{49}\end{array}$ & RCT & $\begin{array}{l}\text { Improve } \\
\text { rational drug } \\
\text { use by } \\
\text { promoting } \\
\text { EB-prescribing }\end{array}$ & $\begin{array}{l}\text { Physicians } \\
(N=250)\end{array}$ & $\begin{array}{l}\text { Comparison feedback, } \\
\text { guideline, educational } \\
\text { bulletin with tips }\end{array}$ & $\begin{array}{l}\text { Median prescription cost } \\
(\$ I I) \text { remained constant in } \\
\text { the intervention group but } \\
\text { increased in the control } \\
\text { group }(p<0.002) \text {. First- } \\
\text { line drug use increased in } \\
\text { the intervention group but } \\
\text { decreased in the control } \\
\text { group }(p<0.01)\end{array}$ & $+/+$ \\
\hline
\end{tabular}


Table 4: Overview of included articles (continued) NR= not reported.

\begin{tabular}{llll}
\hline Author Design & Aim & $\begin{array}{l}\text { Target } \\
\text { group }\end{array}$ & Focus of intervention Main results \\
Year & & (N)
\end{tabular}

(N)

\section{Quality \\ Rigorl}

Relevance

\begin{tabular}{|c|c|c|c|c|c|c|}
\hline $\begin{array}{l}\text { Légaré et } \\
\text { al. } \\
2010^{57}\end{array}$ & RCT & $\begin{array}{l}\text { Decrease } \\
\text { overuse of } \\
\text { antibiotics }\end{array}$ & $\begin{array}{l}\text { Physicians } \\
(N=33)\end{array}$ & $\begin{array}{l}\text { EB workshop, feedback, } \\
\text { reminders of expected } \\
\text { behavior }\end{array}$ & $\begin{array}{l}\text { Intervention group reduced } \\
\text { its immediate use of } \\
\text { antibiotics ( } 49 \text { vs } 33 \% \\
p=0.08 \text { ). Decisional conflict } \\
\text { agreement was stronger in } \\
\text { the intervention group } \\
(p=0.06)\end{array}$ & $+/+$ \\
\hline $\begin{array}{l}\text { Fortuna } \\
\text { et al., } \\
2009^{63}\end{array}$ & RCT & $\begin{array}{l}\text { Decrease in } \\
\text { prescribing drugs }\end{array}$ & $\begin{array}{l}\text { Physicians } \\
(\mathrm{N}=257) \\
\text { other }\end{array}$ & $\begin{array}{l}\text { Computerized alert } \\
\text { with recommendation } \\
\text { supported by EB, } \\
\text { co-payment } \\
\text { information, patient } \\
\text { information regarding } \\
\text { side effects. Interactive } \\
\text { group discussion } \\
\text { regarding barriers to } \\
\text { guideline adherence }\end{array}$ & $\begin{array}{l}\text { RR of prescribing marketed } \\
\text { medication in intervention } \\
\text { group is less than control } \\
\text { group }(0.74[0.58-0.97] \\
p=0.03) .23 .3 \% \text { of } \\
\text { prescriptions that received } \\
\text { alert was altered }\end{array}$ & $+/+$ \\
\hline $\begin{array}{l}\text { Rotman } \\
\text { et al., } \\
1996^{80}\end{array}$ & RCT & $\begin{array}{l}\text { Decrease in } \\
\text { costs of } \\
\text { prescribing }\end{array}$ & $\begin{array}{l}\text { Residents } \\
(\mathrm{N}=37)\end{array}$ & $\begin{array}{l}\text { Cost information, } \\
\text { guideline, alerts with } \\
\text { suggestion }\end{array}$ & $\begin{array}{l}\text { Increased user satisfaction. } \\
\text { No significant differences in } \\
\text { health of economic } \\
\text { outcomes }\end{array}$ & $+/-$ \\
\hline $\begin{array}{l}\text { Smith } \\
\text { et al., } \\
1983^{92}\end{array}$ & RCT & $\begin{array}{l}\text { Stimulate cost } \\
\text { consciousness }\end{array}$ & $\begin{array}{l}\text { Medical } \\
\text { students } \\
(\mathrm{N}=37)\end{array}$ & $\begin{array}{l}\text { Feedback on efficiency, } \\
\text { errors, costs, risk and } \\
\text { discomfort Discussion } \\
\text { with expert }\end{array}$ & $\begin{array}{l}\text { No significant different in six } \\
\text { patient management } \\
\text { problems. Significant } \\
(p<0.05) \text { improvement } \\
\text { overall for student when } \\
\text { comparing pre-test and } \\
\text { post-test }\end{array}$ & $-/-$ \\
\hline $\begin{array}{l}\text { Sussman } \\
\text { et al., } \\
2004^{14}\end{array}$ & $\begin{array}{l}\text { Pre-post } \\
\text { interventional } \\
\text { study }\end{array}$ & $\begin{array}{l}\text { Stimulate the use } \\
\text { of cost-effective } \\
\text { alternatives }\end{array}$ & $\begin{array}{l}\text { Physicians, } \\
\text { primary care } \\
(\mathrm{N}=57)\end{array}$ & $\begin{array}{l}\text { Information for cost- } \\
\text { effective prescribing, } \\
\text { specific suggestions for } \\
\text { change, cost feedback }\end{array}$ & $\begin{array}{l}\text { The use of high cost } \\
\text { decreased from } 38 \% \text { to } 30 \% \\
(p<0.001 \text { ) }\end{array}$ & $+/+$ \\
\hline
\end{tabular}


Table 4: Overview of included articles (continued) NR= not reported.

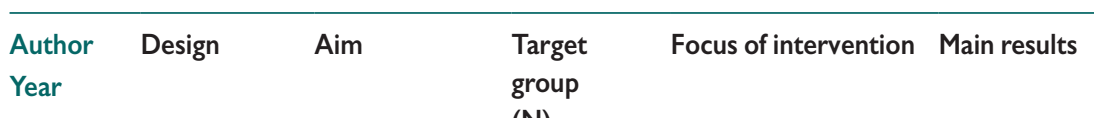

(N)

\begin{tabular}{|c|c|c|c|c|c|c|}
\hline $\begin{array}{l}\text { Post } \\
\text { et al., } \\
2013^{16}\end{array}$ & $\begin{array}{l}\text { Pre-post } \\
\text { interventional } \\
\text { study }\end{array}$ & $\begin{array}{l}\text { Improve cost } \\
\text { awareness } \\
\text { Improve } \\
\text { knowledge of } \\
\text { costs and value }\end{array}$ & $\begin{array}{l}\text { Residents } \\
(\mathrm{N}=83)\end{array}$ & $\begin{array}{l}\text { Feedback cost data, } \\
\text { reflection on ordering }\end{array}$ & $\begin{array}{l}\text { Decreased mean \% error in } \\
\text { cost estimates }(83.1 \% \text { versus } \\
I 3.4 \% p<0.000 I) \text {, attitude } \\
\text { regarding costs significantly } \\
\text { changed }(p<0.05)\end{array}$ & $+/+$ \\
\hline $\begin{array}{l}\text { Das } \\
\text { et al., } \\
2010^{31}\end{array}$ & $\begin{array}{l}\text { Pre-post } \\
\text { interventional } \\
\text { study }\end{array}$ & $\begin{array}{l}\text { Optimize } \\
\text { vitamin } \\
\text { prescribing } \\
\text { decrease } \\
\text { wastage of } \\
\text { money }\end{array}$ & $\begin{array}{l}\text { Physicians } \\
(\mathrm{N}=30)\end{array}$ & $\begin{array}{l}\text { Prescriber focus group, } \\
\text { feedback data and EBM } \\
\text { reflection }\end{array}$ & $\begin{array}{l}\text { Prevalence of vitamin } \\
\text { prescribing, cost of vitamin } \\
\text { prescribing significantly } \\
\text { decreased }(p<0.001)\end{array}$ & $+1+$ \\
\hline $\begin{array}{l}\text { Cammisa } \\
\text { et al., } \\
20 \mathrm{II}^{34}\end{array}$ & $\begin{array}{l}\text { Pre-post } \\
\text { interventional } \\
\text { study }\end{array}$ & $\begin{array}{l}\text { Improve the } \\
\text { quality by } \\
\text { lowering overuse }\end{array}$ & $\begin{array}{l}\text { Physicians } \\
(\mathrm{N}=\mathrm{NR})\end{array}$ & $\begin{array}{l}\text { Expert reflection on } \\
\text { utilization, advice, } \\
\text { guidelines }\end{array}$ & $\begin{array}{l}\text { Post-intervention, } \\
\text { narcotics, muscle relaxants, } \\
\text { MRIs, and spinal injections } \\
\text { decreased }(p<.000 \mathrm{I})\end{array}$ & $+/+$ \\
\hline $\begin{array}{l}\text { Self } \\
\text { et al., } \\
1984^{35}\end{array}$ & $\begin{array}{l}\text { Pre-post } \\
\text { interventional } \\
\text { study (no } \\
\text { control group) }\end{array}$ & $\begin{array}{l}\text { Improve rational } \\
\text { use Lower costs }\end{array}$ & $\begin{array}{l}\text { Medical } \\
\text { students, } \\
\text { residents } \\
(\mathrm{N}=18)\end{array}$ & $\begin{array}{l}\text { Guideline and } \\
\text { indications, cost } \\
\text { information }\end{array}$ & $\begin{array}{l}\text { Prevalence of vitamin } \\
\text { prescribing and contributions } \\
\text { of vitamins in the cost of } \\
\text { drugs prescribed in vitamin } \\
\text { containing prescription } \\
\text { significantly decreased } \\
(p<0.00 \mathrm{I})\end{array}$ & $+/-$ \\
\hline $\begin{array}{l}\text { McKay } \\
\text { et al., } \\
2011^{36}\end{array}$ & $\begin{array}{l}\text { Pre-post } \\
\text { interventional } \\
\text { study }\end{array}$ & $\begin{array}{l}\text { Decrease in } \\
\text { unnecessary } \\
\text { prescribed drugs }\end{array}$ & $\begin{array}{l}\text { Residents, } \\
\text { physicians, } \\
\text { other } \\
(\mathrm{N}=63)\end{array}$ & $\begin{array}{l}\text { Training to provide } \\
\text { patient education, } \\
\text { course on } A B \text { use, } \\
\text { strategies to prescribe } \\
\text { appropriately }\end{array}$ & $\begin{array}{l}\text { Significant }(p=0.013) \\
\text { increase in general } \\
\text { knowledge. Significant } \\
\text { decrease in antibiotics use for } \\
\text { acute bronchitis }(p=0.023) \text {, } \\
\text { all indications }(p=0.019) \text { and } \\
\text { macrolides ( } p<0.001) .4 .5 \% \\
\text { reduction in antibiotics cost } \\
\text { across the province }\end{array}$ & $-/-$ \\
\hline
\end{tabular}


Table 4: Overview of included articles (continued) NR= not reported.

\begin{tabular}{llll}
\hline Author Design & Aim & $\begin{array}{l}\text { Target } \\
\text { group }\end{array}$ & Focus of intervention Main results \\
Year & & (N)
\end{tabular}

(N)

\section{Quality \\ Rigorl \\ Relevance}

\begin{tabular}{|c|c|c|c|c|c|c|}
\hline $\begin{array}{l}\text { Blackstone } \\
\text { et al., } \\
1995^{39}\end{array}$ & $\begin{array}{l}\text { Pre-post } \\
\text { interventional } \\
\text { study }\end{array}$ & $\begin{array}{l}\text { Educate in the } \\
\text { area of cost } \\
\text { awareness and } \\
\text { containment }\end{array}$ & $\begin{array}{l}\text { Residents } \\
(N=2) \\
\text { surgery } \\
(N=2) \\
\text { interns } \\
(N=2)\end{array}$ & $\begin{array}{l}\text { Guidelines and } \\
\text { recommendation, } \\
\text { multidisciplinary } \\
\text { discussions }\end{array}$ & $\begin{array}{l}\text { Total median daily charges of } \\
\text { post-intervention group were } \\
\text { reduced compared to control } \\
\text { group by } \$ 8 \mid 8 / \text { intensive care } \\
\text { unit day }(p=0.0002)\end{array}$ & $-/+$ \\
\hline $\begin{array}{l}\text { Fakih } \\
\text { et al., } \\
2010^{42}\end{array}$ & $\begin{array}{l}\text { Pre-post } \\
\text { interventional } \\
\text { study }\end{array}$ & $\begin{array}{l}\text { Reduce } \\
\text { unnecessary } \\
\text { urinary catheter } \\
\text { placements }\end{array}$ & $\begin{array}{l}\text { Residents } \\
(N=30), \\
\text { physicians } \\
(N=39)\end{array}$ & $\begin{array}{l}\text { EB clinical guideline and } \\
\text { indications, pocket card, } \\
\text { reflection }\end{array}$ & $\begin{array}{l}\text { Intervention caused an overall } \\
\text { reduction in UC utilization } \\
\text { from } 16.4 \% \text { to I } 3 \% \\
(p=0.018) \text {. Physicians } \\
\text { ordered } 40 \% \text { fewer UCs } \\
\text { post-intervention }\end{array}$ & $+/+$ \\
\hline $\begin{array}{l}\text { Sleath } \\
\text { et al., } \\
1997^{45}\end{array}$ & $\begin{array}{l}\text { Pre-post } \\
\text { interventional } \\
\text { study }\end{array}$ & $\begin{array}{l}\text { Stimulate } \\
\text { appropriate care } \\
\text { reduce adverse } \\
\text { events }\end{array}$ & $\begin{array}{l}\text { Physicians } \\
(\mathrm{N}=90) \\
\text { other }\end{array}$ & $\begin{array}{l}\text { Drug use review, } \\
\text { educational letter with } \\
\text { EB suggestion for } \\
\text { physicians, and } \\
\text { physicians and } \\
\text { pharmacists }\end{array}$ & $\begin{array}{l}\text { Decrease in mean monthly } \\
\text { costs for } B 2 \text {-agonist ( } \$ 74,96 \\
\text { vs } \$ 61,14 \text { ) } \mathrm{P}<0.05 \text {.Average } \\
\text { monthly cost per patient of all } \\
\text { asthma medications } \\
\text { decreased and cost for } \\
\text { asthma-related use of the } \\
\text { health system increased (not } \\
\text { significant) }\end{array}$ & $-/-$ \\
\hline $\begin{array}{l}\text { Bhatia } \\
\text { et al., } \\
2013^{48}\end{array}$ & $\begin{array}{l}\text { Pre-post } \\
\text { interventional } \\
\text { study }\end{array}$ & $\begin{array}{l}\text { Lower the } \\
\text { proportion of } \\
\text { inappropriate } \\
\text { echocardiograms }\end{array}$ & $\begin{array}{l}\text { Medical } \\
\text { students, } \\
\text { residents, } \\
\text { physicians } \\
(\mathrm{N}=\mathrm{NR})\end{array}$ & $\begin{array}{l}\text { AUC lecture, pocket } \\
\text { card of tips, utilization } \\
\text { and feedback on } \\
\text { appropriateness }\end{array}$ & $\begin{array}{l}\text { Pre-post-intervention a } \\
26 \% \text { reduction in number } \\
\text { ofTTE ordered per day } \\
(p<0.00 \text { I). Proportion of } \\
\text { inappropriate TTE was } \\
\text { significantly lower ( } 5 \% \text { vs. } \\
\text { I3\%, } p<0.00 \text { I) and } \\
\text { proportion of appropriate } \\
\text { TTE was significantly higher } \\
(93 \% \text { vs. } 84 \%, p<0.00 \text { I) }\end{array}$ & $+/+$ \\
\hline $\begin{array}{l}\text { Larmour } \\
\text { et al., } \\
2011^{50}\end{array}$ & $\begin{array}{l}\text { Pre-post } \\
\text { interventional } \\
\text { study }\end{array}$ & $\begin{array}{l}\text { Maintain quality } \\
\text { while containing } \\
\text { healthcare costs }\end{array}$ & $\begin{array}{l}\text { Residents, } \\
\text { physicians } \\
(N=N R)\end{array}$ & $\begin{array}{l}\text { Guidelines, academic } \\
\text { detailing, promotion } \\
\text { among staff }\end{array}$ & $\begin{array}{l}\text { A total of } \$ 3.16 \text { million was } \\
\text { saved.The annual savings } \\
\text { increased each year }\end{array}$ & $+/+$ \\
\hline
\end{tabular}


Table 4: Overview of included articles (continued) NR= not reported.

\begin{tabular}{|c|c|c|c|c|c|c|}
\hline \multirow{2}{*}{$\begin{array}{l}\text { Author } \\
\text { Year }\end{array}$} & \multirow[t]{2}{*}{ Design } & \multirow[t]{2}{*}{ Aim } & \multirow{2}{*}{$\begin{array}{l}\text { Target } \\
\text { group } \\
(\mathrm{N})\end{array}$} & \multirow[t]{2}{*}{ Focus of intervention } & \multirow[t]{2}{*}{ Main results } & \multirow{2}{*}{$\begin{array}{c}\text { Quality } \\
\text { Rigor/ } \\
\text { Relevance }\end{array}$} \\
\hline & & & & & & \\
\hline $\begin{array}{l}\text { Phillips } \\
\text { et al., } \\
1986^{51}\end{array}$ & $\begin{array}{l}\text { Pre-post } \\
\text { interventional } \\
\text { study (no } \\
\text { control group) }\end{array}$ & Cost saving & $\begin{array}{l}\text { Residents, } \\
\text { physicians } \\
(\mathrm{N}=\mathrm{NR}) \\
\text { other }\end{array}$ & $\begin{array}{l}\text { Newsletter with price } \\
\text { information and } \\
\text { suggestion for } \\
\text { improvement }\end{array}$ & $\begin{array}{l}\text { No significant increase or } \\
\text { decrease in \% of IV doses in } \\
\text { main three categories }\end{array}$ & $-/-$ \\
\hline $\begin{array}{l}\text { Ziskind } \\
\text { et al., } \\
1994^{52}\end{array}$ & $\begin{array}{l}\text { Pre-post } \\
\text { interventional } \\
\text { study, time } \\
\text { series }\end{array}$ & $\begin{array}{l}\text { Increased use of } \\
\text { cost-effective } \\
\text { contrast }\end{array}$ & $\begin{array}{l}\text { Physicians } \\
\text { (N=NR) }\end{array}$ & $\begin{array}{l}\text { Guidelines with } \\
\text { suggestions, educational } \\
\text { memo on overuse, cost } \\
\text { feedback }\end{array}$ & $\begin{array}{l}59 \% \text { decrease in use of high } \\
\text { cost contrast, } \$ 143 \text { decrease } \\
\text { in contrast expenditure per } \\
\text { patient. No adverse events }\end{array}$ & $+/+$ \\
\hline $\begin{array}{l}\text { Shane } \\
\text { et al., } \\
1994^{53}\end{array}$ & $\begin{array}{l}\text { Pre-post } \\
\text { interventional } \\
\text { study (no } \\
\text { control) }\end{array}$ & $\begin{array}{l}\text { Stimulate } \\
\text { appropriate and } \\
\text { cost-effective } \\
\text { care }\end{array}$ & $\begin{array}{l}\text { Physicians } \\
\text { (N=NR) }\end{array}$ & $\begin{array}{l}\text { Expert reflection, } \\
\text { guidelines }\end{array}$ & $\begin{array}{l}\text { Cost of therapy decreased } \\
\text { from } \$ 983 \text { to } \$ 729(-26 \%) \\
\text { and from } \$ 737 \text { to } \$ 294 \\
(-60 \%)\end{array}$ & $-/-$ \\
\hline $\begin{array}{l}\text { Okpara } \\
\text { et al., } \\
1995^{56}\end{array}$ & $\begin{array}{l}\text { Pre-post } \\
\text { interventional } \\
\text { study }\end{array}$ & $\begin{array}{l}\text { Stimulate } \\
\text { appropriate } \\
\text { and cost- } \\
\text { effective } \\
\text { prescribing }\end{array}$ & $\begin{array}{l}\text { Residents, } \\
\text { physicians } \\
(\mathrm{N}=78) \\
\text { other }\end{array}$ & $\begin{array}{l}\text { EB criteria, expert } \\
\text { recommendations for } \\
\text { improvement }\end{array}$ & $\begin{array}{l}66 \text { physicians }(84.6 \%) \\
\text { accepted the } \\
\text { recommendation, estimated } \\
\text { cost savings } \$ 12.935 \text { ( } 2 \\
\text { months) }\end{array}$ & $-/+$ \\
\hline $\begin{array}{l}\text { Yang } \\
\text { et al., } \\
2014^{62}\end{array}$ & $\begin{array}{l}\text { Pre-post } \\
\text { interventional } \\
\text { study }\end{array}$ & $\begin{array}{l}\text { Promote } \\
\text { adherence to } \\
\text { rational drug use } \\
\text { guideline in } A B- \\
\text { prophylaxis. }\end{array}$ & $\begin{array}{l}\text { Physicians } \\
(N=N R)\end{array}$ & $\begin{array}{l}\text { Integration of guideline } \\
\text { in ordering system, } \\
\text { educational round by } \\
\text { pharmacy department }\end{array}$ & $\begin{array}{l}\text { Significant increase in } \\
\text { proportion of cefazolin } \\
(p<0.05) \text {. Significant } \\
\text { decrease in average hospital } \\
\text { stay }(p<0.05) \text {. No significant } \\
\text { change in surgical site } \\
\text { infections and adverse drug } \\
\text { events or reactions }\end{array}$ & $+/+$ \\
\hline $\begin{array}{l}\text { Thakkar } \\
\text { et al., } \\
2015^{74}\end{array}$ & $\begin{array}{l}\text { Pre-post } \\
\text { interventional } \\
\text { study }\end{array}$ & $\begin{array}{l}\text { Reduce } \\
\text { laboratory tests }\end{array}$ & $\begin{array}{l}\text { Physicians, } \\
\text { residents, } \\
\text { other } \\
(\mathrm{N}=\mathrm{NR})\end{array}$ & $\begin{array}{l}\text { Educational session } \\
\text { with discussion, } \\
\text { distribution of } \\
\text { educational flyers and } \\
\text { informative emails } \\
\text { regarding I) utility of } \\
\text { test. } 2 \text { ) impact on } \\
\text { costs and 3) } \\
\text { recommendations }\end{array}$ & $\begin{array}{l}\text { Reduction in the number of } \\
\text { overall test orders ( } 4.14 \text { vs } \\
3.79 \text { per patient per day, } p \\
=0.001) \text {. Cost reduction of } \\
\$ 6.33 \text { per patient per day }\end{array}$ & $+/+$ \\
\hline
\end{tabular}


Table 4: Overview of included articles (continued) NR= not reported.

\begin{tabular}{llll}
\hline Author Design & Aim & $\begin{array}{l}\text { Target } \\
\text { group }\end{array}$ & Focus of intervention Main results \\
Year & & (N)
\end{tabular}

(N)

\section{Quality \\ Rigorl \\ Relevance}

\begin{tabular}{lllll}
\hline Krinsley & Pre-post & Safely decrease & Residents & $\begin{array}{l}\text { Reflective questions on } \\
\text { et al., }\end{array}$ \\
interventional & $\begin{array}{l}\text { the utilization of } \\
(\mathrm{N}=\mathrm{NR})\end{array}$ & ordering form \\
$2003^{78}$ & study & imaging & &
\end{tabular}

CXR utilization rate

$-/+$ decreased by $22.5 \%$ during study period. This resulted in a $\$ 109.968$ cost savings, which were not associated with any adverse clinical outcomes

\begin{tabular}{|c|c|c|c|c|c|c|}
\hline $\begin{array}{l}\text { Zeleznik } \\
\text { et al., } \\
1979^{84}\end{array}$ & $\begin{array}{l}\text { Pre-post } \\
\text { interventional } \\
\text { study }\end{array}$ & $\begin{array}{l}\text { Contain costs } \\
\text { and reduce } \\
\text { inappropriate } \\
\text { care }\end{array}$ & $\begin{array}{l}\text { Medical } \\
\text { students } \\
(\mathrm{N}=54)\end{array}$ & $\begin{array}{l}\text { Case discussion on } \\
\text { appropriateness and } \\
\text { costs }\end{array}$ & $\begin{array}{l}\text { Intervention resulted in } \\
\text { increased knowledge (6\%) } \\
\text { and attitude toward } \\
\text { usefulness and costs }\end{array}$ & $-1+$ \\
\hline $\begin{array}{l}\text { Lyle } \\
\text { et al., } \\
1979^{86}\end{array}$ & $\begin{array}{l}\text { Pre-post } \\
\text { interventional } \\
\text { study }\end{array}$ & $\begin{array}{l}\text { Promote } \\
\text { awareness of } \\
\text { costs Decrease } \\
\text { overutilization }\end{array}$ & $\begin{array}{l}\text { Medical } \\
\text { students, } \\
\text { residents } \\
(\mathrm{N}=\mathrm{NR})\end{array}$ & Utilization feedback & $\begin{array}{l}21 \% \text { reduction in average } \\
\text { lengths of stay, cost per } \\
\text { admission rose } 4.3 \% \text { per year } \\
\text { in comparison to a rise of } \\
14.5 \% \text { in other services }\end{array}$ & +/+ \\
\hline $\begin{array}{l}\text { Spiegel } \\
\text { et al., } \\
1982^{15}\end{array}$ & $\begin{array}{l}\text { Quasi- } \\
\text { experimental } \\
\text { design }\end{array}$ & $\begin{array}{l}\text { Improve ability } \\
\text { to choose } \\
\text { appropriately }\end{array}$ & $\begin{array}{l}\text { Medical } \\
\text { students } \\
(N=|5|)\end{array}$ & $\begin{array}{l}\text { Reflection on orders, } \\
\text { evaluate net benefit of } \\
\text { decisions, cost } \\
\text { information, EB } \\
\text { information on medical } \\
\text { topics }\end{array}$ & $\begin{array}{l}\text { Intervention group more } \\
\text { often agreed with experts } \\
\text { that a test was not useful } \\
(93 \% \text { versus } 73 \% p=0.004) \text {. } \\
\text { Control group called for } 30 \% \\
\text { more hospitalizations }\end{array}$ & +/+ \\
\hline $\begin{array}{l}\text { Schroeder } \\
\text { et al., } \\
1984^{28}\end{array}$ & $\begin{array}{l}\text { Quasi- } \\
\text { experimental } \\
\text { design }\end{array}$ & $\begin{array}{l}\text { Eliminate } \\
\text { unwarranted } \\
\text { orders } \\
\text { Reduce charges }\end{array}$ & $\begin{array}{l}\text { Medical } \\
\text { students, } \\
\text { residents } \\
(\mathrm{N}=43)\end{array}$ & $\begin{array}{l}\text { Cost information, } \\
\text { indications, utilization } \\
\text { and cost feedback }\end{array}$ & $\begin{array}{l}\text { Significant difference in LOS } \\
\text { (pre-intervention } 5.8 \text { vs } \\
\text { post-intervention } 6.5 p<0.01 \text { ). } \\
\text { Cost reduction }-\$ 117 \text { and } \\
-\$ 62 \text { (non-significant) }\end{array}$ & $+/+$ \\
\hline $\begin{array}{l}\text { Gitelis } \\
\text { et al., } \\
2015^{29}\end{array}$ & $\begin{array}{l}\text { Quasi- } \\
\text { experimental }\end{array}$ & $\begin{array}{l}\text { Decrease in } \\
\text { costs Lower the } \\
\text { use of disposable } \\
\text { materials }\end{array}$ & $\begin{array}{l}\text { Physicians } \\
(N=15)\end{array}$ & $\begin{array}{l}\text { Presentation on costs } \\
\text { of disposable } \\
\text { equipment, cost by } \\
\text { individual surgeon, } \\
\text { cost-effective } \\
\text { alternatives }\end{array}$ & $\begin{array}{l}10 \% \text { reduction in average cost } \\
(p<0.001) \text {, no difference in } \\
\text { readmissions/reoperations. }\end{array}$ & $-/-$ \\
\hline
\end{tabular}


Table 4: Overview of included articles (continued) NR= not reported.

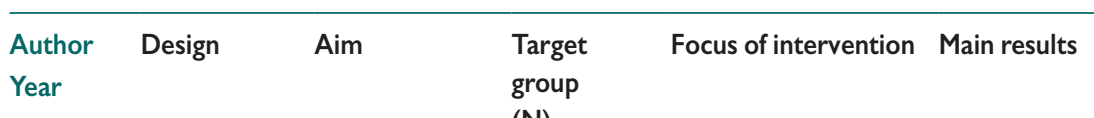

(N)

\begin{tabular}{llll}
\hline Vignes- & Quasi- & Reduce costs & Physicians \\
waran & experimental & Lower use of & $(\mathrm{N}=10)$ \\
et al., & & $\begin{array}{l}\text { disposable } \\
\text { materials }\end{array}$ & \\
$2015^{30}$ & &
\end{tabular}

Presentation on costs

of disposable

equipment, cost by

individual surgeon,

cost-effective

alternatives

$\begin{array}{llll}\text { Attali } & \text { Quasi- } & \begin{array}{l}\text { Decrease in } \\ \text { et al., }\end{array} & \text { Residents, } \\ 2006^{72} & \text { experimental } & \begin{array}{l}\text { inappropriate } \\ \text { laboratory } \\ \text { testing }\end{array} & (\mathrm{N}=\mathrm{II})\end{array}$

Zimmerman Quasi-

et al., experimental

$1994^{75}$

design

Stimulate

appropriateness $\quad(\mathrm{N}=\mathrm{NR})$

Increase efficacy

$\begin{array}{ll}\text { Zunker } & \text { Quasi- } \\ \text { et al., } & \text { experimental } \\ 2000^{76} & \text { design } \\ & \text { (control) }\end{array}$

Increase in

cost-effective

prescribing

Rudy

et al.,

$200 I^{79}$

Quasi-

experimental

design

Reduce cost

of care

Landgren

et al.,

1988'

$\begin{array}{lll}\text { Quasi- } & \begin{array}{l}\text { Improve the } \\ \text { experimental }\end{array} & \begin{array}{l}\text { Physicians } \\ \text { appropriateness } \\ \text { of prescribing }\end{array}\end{array}$

Physicians

Residents

$(\mathrm{N}=23)$

Lecture on excessive or
inappropriate ordering
of tests, supervised
ordering
DUR letter containing evidence, utilization feedback

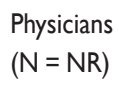

EBM information, expert feedback, utilization feedback

Workshop on cost effectiveness, decisionmaking with/without charges

\section{Campaign, feedback} with discussion, satirical video to trigger discussion, visit of academic representative to explain campaign and answer questions
Decrease of 97.365 tests during 3-year period, saving $\$ 1.914 .194$. No difference in readmission rate or number of diagnoses of conditions between departments
Decrease in monthly drug dose $(p<0.10)$. Reduction in total costs $\$ 17.76$ per month per patient $(p<0.05)$

Average cost reduction of $\$ 228$ (-21\%) for laparoscopic hernia repair $(p<0.00 \mathrm{I})$
$-1+$

(1)

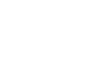

Antibiotic courses assessed as + +/satisfactory in terms of duration increased after the first intervention campaign. No significant changes in prescribing occurred in the control hospitals 
Table 4: Overview of included articles (continued) NR= not reported.

\begin{tabular}{llll}
\hline Author Design & Aim & $\begin{array}{l}\text { Target } \\
\text { group }\end{array}$ & Focus of intervention Main results \\
Year & & (N)
\end{tabular}

(N)

\begin{tabular}{|c|c|c|c|c|c|c|}
\hline $\begin{array}{l}\text { Sommers } \\
\text { et al., } \\
2012^{22}\end{array}$ & $\begin{array}{l}\text { Mixed } \\
\text { methods (RCT, } \\
\text { open } \\
\text { comments) }\end{array}$ & $\begin{array}{l}\text { Improve } \\
\text { awareness of } \\
\text { how decisions } \\
\text { influence costs }\end{array}$ & $\begin{array}{l}\text { Medical } \\
\text { students, } \\
\text { residents } \\
(\mathrm{N}=47)\end{array}$ & $\begin{array}{l}\text { Cost feedback, discuss } \\
\text { strategy to reduce } \\
\text { costs, cost information }\end{array}$ & $\begin{array}{l}\text { Non-significant reduction of } \\
\$ 69 \text { per admission in } \\
\text { intervention group }(p=0.92) \text {, } \\
\text { significant reduction in } \\
\text { laboratory test price }(-\$ 163 \\
p=0.046) \text {. Increased } \\
\text { awareness among residents }\end{array}$ & $+/+$ \\
\hline $\begin{array}{l}\text { McPhee } \\
\text { et al., } \\
1984^{58}\end{array}$ & $\begin{array}{l}\text { Mixed methods } \\
\text { (Quasi- } \\
\text { experimental } \\
\text { design, } \\
\text { observation and } \\
\text { discussion) }\end{array}$ & $\begin{array}{l}\text { Control rise in } \\
\text { costs reduce } \\
\text { unnecessary } \\
\text { ordering }\end{array}$ & $\begin{array}{l}\text { Medical } \\
\text { students } \\
\text { residents } \\
(\mathrm{N}=\mathrm{NR})\end{array}$ & $\begin{array}{l}\text { Reviewing medical } \\
\text { records, cost-feedback, } \\
\text { price information }\end{array}$ & $\begin{array}{l}\text { Moderate to high acceptance } \\
\text { of intervention. Impact on use } \\
\text { of hospital service was } \\
\text { modest (no statistical } \\
\text { analysis) }\end{array}$ & $-/+$ \\
\hline $\begin{array}{l}\text { Sicotte } \\
\text { et al., } \\
1996^{77}\end{array}$ & $\begin{array}{l}\text { Mixed } \\
\text { methods } \\
\text { (Pre-post } \\
\text { interventional } \\
\text { study and } \\
\text { interviews) }\end{array}$ & $\begin{array}{l}\text { Reduce } \\
\text { inappropriate } \\
\text { resource use }\end{array}$ & $\begin{array}{l}\text { Physicians } \\
(N=20)\end{array}$ & $\begin{array}{l}\text { Utilization feedback, } \\
\text { reflection on feedback, } \\
\text { involvement of head of } \\
\text { department }\end{array}$ & $\begin{array}{l}\text { Mixed results on behavior } \\
\text { modification (no change, } \\
\text { increase, decrease in resource } \\
\text { utilization) }\end{array}$ & $+/+$ \\
\hline
\end{tabular}

Articles that addressed I factor

\begin{tabular}{|c|c|c|c|c|c|}
\hline $\begin{array}{l}\text { Davidoff } \\
\text { et al., } \\
1989^{19}\end{array}$ & RCT & $\begin{array}{l}\text { Improve } \\
\text { decision-making }\end{array}$ & $\begin{array}{l}\text { Residents } \\
(\mathrm{N}=24)\end{array}$ & $\begin{array}{l}\text { Course teaching cost- } \\
\text { containment topics or } \\
\text { probability theory }\end{array}$ & $\begin{array}{l}\text { Significantly fewer orders } \\
\text { for laboratory tests } \\
\text { compared to placebo group } \\
(p=0.032,16 \%)\end{array}$ \\
\hline $\begin{array}{l}\text { Marconi } \\
\text { et al., } \\
2010^{38}\end{array}$ & $\mathrm{RCT}$ & $\begin{array}{l}\text { Improve cost } \\
\text { awareness } \\
\text { Increase quality } \\
\text { and } \\
\text { cost } \\
\text { containment }\end{array}$ & $\begin{array}{l}\text { Residents } \\
(\mathrm{N}=80)\end{array}$ & EB guidelines & $\begin{array}{l}\text { Significant increase in } \\
\text { average number of } \\
\text { correct answers on the } \\
\text { pre-/post-intervention } \\
\text { questionnaires compared } \\
\text { with control group } \\
(p<0.00 \mathrm{I})\end{array}$ \\
\hline
\end{tabular}


Table 4: Overview of included articles (continued) NR= not reported.

\begin{tabular}{|c|c|c|c|c|c|c|}
\hline Author & Design & Aim & Target & Focus of intervention & Main results & Quality \\
\hline Year & & & $\begin{array}{l}\text { group } \\
(\mathrm{N})\end{array}$ & & & $\begin{array}{c}\text { Rigor/ } \\
\text { Relevance }\end{array}$ \\
\hline
\end{tabular}

\begin{tabular}{|c|c|c|c|}
\hline $\begin{array}{l}\text { Légaré } \\
\text { et al., } \\
2012^{58}\end{array}$ & RCT & $\begin{array}{l}\text { Reduce overuse } \\
\text { of antibiotics }\end{array}$ & $\begin{array}{l}\text { Residents, } \\
\text { physicians } \\
(\mathrm{N}=|5|)\end{array}$ \\
\hline
\end{tabular}

Tutorial on patientcommunication, workshop to implement after consultation was higher lessons in practice

Percentage of patients deciding to use antibiotics in the control group (52.2\%) + $(N=|5|)$ compared with the intervention group (27.2\%) (absolute difference 25.0\%). Intervention was associated with patients taking a more active role in decision-making $(Z=3.9, p<0.00 I)$

\begin{tabular}{|c|c|c|c|c|}
\hline $\begin{array}{l}\text { Braido } \\
\text { et al., } \\
2012^{59}\end{array}$ & RCT & $\begin{array}{l}\text { Improve } \\
\text { guideline } \\
\text { knowledge and } \\
\text { adherence }\end{array}$ & $\begin{array}{l}\text { Physicians } \\
(\mathrm{N}=60)\end{array}$ & $\begin{array}{l}\text { Economic analysis } \\
\text { course regarding } \\
\text { prescribing and } \\
\text { resource utilization }\end{array}$ \\
\hline
\end{tabular}

Knowledge improved $+/-$ significantly after training (correct answers to key questions $+13 \%, p<0.001$ ). Cost containment (trained general practitioners $+0.5 \%$ vs controls $+18.8 \%$ ) and greater attention to diagnosis and monitoring (increase in spirometry $+63.4 \%, p<0.0$ I)

\begin{tabular}{|c|c|c|c|c|}
\hline $\begin{array}{l}\text { Bates } \\
\text { et al., } \\
1999^{73}\end{array}$ & RCT & $\begin{array}{l}\text { Reduce the } \\
\text { amount of } \\
\text { redundant test } \\
\text { ordering }\end{array}$ & $\begin{array}{l}\text { Physicians } \\
(\mathrm{N}=\mathrm{NR})\end{array}$ & $\begin{array}{l}\text { Feedback alert for } \\
\text { redundant tests }\end{array}$ \\
\hline $\begin{array}{l}\text { Collins } \\
\text { et al., } \\
1997^{89}\end{array}$ & RCT & $\begin{array}{l}\text { Stimulate } \\
\text { accuracy and } \\
\text { appropriateness } \\
\text { of prescribing }\end{array}$ & $\begin{array}{l}\text { Physicians } \\
(\mathrm{N}=285), \\
\text { Pharmacists, } \\
(\mathrm{N}=304) \\
\text { other }\end{array}$ & $\begin{array}{l}\text { Patient-specific } \\
\text { information on } \\
\text { inappropriate } \\
\text { prescribed drugs and } \\
\text { guideline information }\end{array}$ \\
\hline $\begin{array}{l}\text { Ferris } \\
\text { et al., } \\
2005^{90}\end{array}$ & RCT & $\begin{array}{l}\text { Improve quality } \\
\text { and decrease } \\
\text { costs of care }\end{array}$ & $\begin{array}{l}\text { Physicians } \\
(\mathrm{N}=65 \mathrm{I})\end{array}$ & $\begin{array}{l}\text { Case-based } \\
\text { recommendation for } \\
\text { prescribing / tests }\end{array}$ \\
\hline
\end{tabular}

Reminders resulted in $69 \% \quad$-/+ test cancellation and significantly less $(p<0.001)$ redundant test ordered in intervention group

Physicians control group spent \$37.0I per patient more on dipyridamole $(p<0.025)$ than physicianspharmacists intervention group

Change in medication $\quad+$ /+ prescribing and diagnostic test use. Insignificant change in patient outcomes and costs of care 
Table 4: Overview of included articles (continued) NR= not reported.

\begin{tabular}{llll}
\hline Author Design & Aim & $\begin{array}{l}\text { Target } \\
\text { group }\end{array}$ & Focus of intervention Main results \\
Year & & (N)
\end{tabular}

(N)

\section{Quality \\ Rigorl}

Relevance

\begin{tabular}{lllll}
\hline Chanda- & Pre-post & Stimulate cost & Residents & Costs pocket card \\
warkar & interventional & awareness & $(\mathrm{N}=53)$ & \\
et al., & study & & \\
$2007^{17}$ & & &
\end{tabular}

Difference found between

$+/+$

baseline cost estimates and

actual cost of treatment $(p=$

0.03). Improvement between

cost estimates before and

after intervention $(p=.002)$

$\begin{array}{llll}\text { Ellemdin } & \text { Pre-post } & \text { Reduce the costs } & \text { Physicians } \\ \text { et al., } & \text { interventional } & \text { of excessive } & (\mathrm{N}=434) \\ 2011^{18} & \text { study } & \text { laboratory tests } & \end{array}$

Ordering costs, write Mean cost per admitted

$+/+$

costs on ordering form patient decreased (27\%).

Mean cost per day in the

intervention group decreased

$(36 \%)$

$\begin{array}{llll}\text { Englander } & \text { Pre-post } & \text { Stimulate care } & \text { Residents } \\ \text { et al., } & \text { interventional } & \text { that is of optimal } & (\mathrm{N}=\mathrm{NR}) \\ 2006^{20} & \text { study } & \text { value } & \end{array}$

\begin{tabular}{|c|c|c|c|}
\hline $\begin{array}{l}\text { Stuebing } \\
\text { et al., } \\
\left.201\right|^{21}\end{array}$ & $\begin{array}{l}\text { Pre-post } \\
\text { interventional } \\
\text { study }\end{array}$ & $\begin{array}{l}\text { Reduce } \\
\text { unnecessary } \\
\text { orders }\end{array}$ & $\begin{array}{l}\text { Residents } \\
(\mathrm{N}=\mathrm{NR})\end{array}$ \\
\hline $\begin{array}{l}\text { Hart } \\
\text { et al., } \\
1997^{23}\end{array}$ & $\begin{array}{l}\text { Pre-post } \\
\text { interventional } \\
\text { study }\end{array}$ & $\begin{array}{l}\text { Reduce } \\
\text { unnecessary } \\
\text { prescribing }\end{array}$ & $\begin{array}{l}\text { Physicians } \\
(N=60)\end{array}$ \\
\hline
\end{tabular}

Knowledge of costs

Use of iSTAT increased from -/savings, meeting regarding cost savings and system-changes for per year online ordering $40 \%$ to $98 \%$ post-intervention. Estimated savings of $\$ 549.780$ Feedback cost data

Decreased cost per patient per day correlation coefficient $=-0.76(p=0.002)$

Cost information

Awareness of drug costs $+/+$ affects physicians significantly; family physicians preferred less expensive drugs even before the intervention

\begin{tabular}{|c|c|c|c|}
\hline $\begin{array}{l}\text { Willens } \\
\text { et al., } \\
2013^{25}\end{array}$ & $\begin{array}{l}\text { Pre-post } \\
\text { interventional } \\
\text { study }\end{array}$ & $\begin{array}{l}\text { Increase } \\
\text { appropriateness } \\
\text { of utilization }\end{array}$ & $\begin{array}{l}\text { Physicians } \\
(\mathrm{N}=28)\end{array}$ \\
\hline $\begin{array}{l}\text { Qureshi } \\
\text { et al., } \\
201^{26}\end{array}$ & $\begin{array}{l}\text { Pre-post } \\
\text { interventional } \\
\text { design (no } \\
\text { control group) }\end{array}$ & $\begin{array}{l}\text { Improve the } \\
\text { quality of drug } \\
\text { prescriptions }\end{array}$ & $\begin{array}{l}\text { Physicians } \\
(\mathrm{N}=12)\end{array}$ \\
\hline
\end{tabular}
AUC, EB lecture
No significant change in followed by discussion, reminders

Guidelines, prescribing- Increase on prescribing course with health knowledge (all items $p<0.02$ ). economy topics, group discussions

Decreased number of $A B$ prescription, decreased number of prescriptions with multiple drugs 
Table 4: Overview of included articles (continued) NR= not reported.

\begin{tabular}{llll}
\hline Author & Design & Aim & $\begin{array}{l}\text { Target } \\
\text { group }\end{array}$ \\
Year & & Focus of intervention Main results
\end{tabular}

(N)

\section{Quality \\ Rigor/ \\ Relevance}

\begin{tabular}{|c|c|c|c|c|c|c|}
\hline $\begin{array}{l}\text { Whiteside } \\
\text { et al., } \\
1987^{33}\end{array}$ & $\begin{array}{l}\text { Pre-post } \\
\text { interventional } \\
\text { study }\end{array}$ & $\begin{array}{l}\text { Stimulate } \\
\text { appropriate } \\
\text { prescribing }\end{array}$ & $\begin{array}{l}\text { Physicians } \\
(\mathrm{N}=94) \\
\text { residents }\end{array}$ & $\begin{array}{l}\text { Guideline, EB letter, } \\
\text { price information }\end{array}$ & $\begin{array}{l}\text { Appropriateness increased to } \\
58 \%(p<0.000 I) \text {. Change from } \\
\text { second-generation } A B \text { to } \\
\text { first-generation } A B \text { in } 28 \% \text { of } \\
\text { cases }\end{array}$ & $-/+$ \\
\hline $\begin{array}{l}\text { Poppleton } \\
\text { et al., } \\
2003^{44}\end{array}$ & $\begin{array}{l}\text { Pre-post } \\
\text { interventional } \\
\text { design }\end{array}$ & $\begin{array}{l}\text { Maintain } \\
\text { excellence and } \\
\text { efficiency } \\
\text { Decrease costs }\end{array}$ & $\begin{array}{l}\text { Physicians } \\
(N=N R)\end{array}$ & $\begin{array}{l}\text { Clinical practice } \\
\text { guideline and education } \\
\text { regarding the use }\end{array}$ & $\begin{array}{l}\text { LOS decreased by }>\mid \text { day. } 25 \% \\
\text { reduction in charges. No } \\
\text { statistical analysis }\end{array}$ & $+/-$ \\
\hline $\begin{array}{l}\text { James } \\
\text { et al., } \\
2014^{60}\end{array}$ & $\begin{array}{l}\text { Pre-post } \\
\text { interventional } \\
\text { study }\end{array}$ & $\begin{array}{l}\text { Promote switch } \\
\text { from IV to oral } \\
\text { drugs }\end{array}$ & $\begin{array}{l}\text { Physicians } \\
(N=50)\end{array}$ & $\begin{array}{l}\text { Oral presentation } \\
\text { regarding guideline and } \\
\text { bioavailability of drugs, } \\
\text { distribution of } \\
\text { pamphlets regarding } \\
\text { criteria for switch }\end{array}$ & $\begin{array}{l}\text { Increased knowledge } \\
(23.5-28.7 \%) \text { of indication for } \\
\text { switch. Mean cost IV-therapy } \\
\text { decreased (pre-intervention } \\
€ 44.63 \text { post-intervention } \\
€ 28.74, p=0.021 \text { ), overall } \\
\text { drug costs decrease (pre- } \\
\text { intervention } € 7419 \text { post- } \\
\text { intervention } € 4733, p=0.032 \text { ) }\end{array}$ & $-/-$ \\
\hline $\begin{array}{l}\text { Pasquale } \\
\text { et al., } \\
2004^{66}\end{array}$ & $\begin{array}{l}\text { Pre-post } \\
\text { interventional } \\
\text { study }\end{array}$ & $\begin{array}{l}\text { Improve care } \\
\text { Lower overall } \\
\text { costs }\end{array}$ & $\begin{array}{l}\text { Physicians, } \\
\text { other } \\
(N=N R)\end{array}$ & $\begin{array}{l}\text { Expert reflection } \\
\text { regarding inappropriate } \\
\text { ordering with } \\
\text { suggestion }\end{array}$ & $\begin{array}{l}77 \% \text { of suggestions were } \\
\text { accepted, estimated cost } \\
\text { saving } \$ 124.480 \text { (8-months) }\end{array}$ & $+/+$ \\
\hline $\begin{array}{l}\text { Gist } \\
\text { et al., } \\
1997^{85}\end{array}$ & $\begin{array}{l}\text { Pre-post } \\
\text { interventional } \\
\text { study }\end{array}$ & $\begin{array}{l}\text { Reduce } \\
\text { unnecessary } \\
\text { breast biopsies } \\
\text { Promote early } \\
\text { detection of } \\
\text { breast cancer }\end{array}$ & $\begin{array}{l}\text { Physicians } \\
(N=2 I)\end{array}$ & $\begin{array}{l}\text { EB clinical algorithm } \\
\text { with follow-up mailing }\end{array}$ & $\begin{array}{l}\text { Effective in reducing the } \\
\text { incidence of unnecessary } \\
\text { surgical procedures }\end{array}$ & $-/-$ \\
\hline $\begin{array}{l}\text { Elligsen } \\
\text { et al., } \\
2012^{40}\end{array}$ & $\begin{array}{l}\text { Quasi- } \\
\text { experimental }\end{array}$ & $\begin{array}{l}\text { Decrease } \\
\text { inappropriate } \\
\text { use of } \\
\text { antimicrobials }\end{array}$ & $\begin{array}{l}\text { Physicians, } \\
\text { other } \\
(\mathrm{N}=6)\end{array}$ & $\begin{array}{l}\text { Expert reflection on } \\
\text { therapy, feedback to } \\
\text { physician }\end{array}$ & $\begin{array}{l}\text { Significant decrease in } \\
\text { experimental group for: } \\
\text { monthly broad-spectrum } \\
\text { antibiotic use }(p<0.00 \text { I), days } \\
\text { of therapy }(p=0.0054)\end{array}$ & $+/-$ \\
\hline
\end{tabular}


Table 4: Overview of included articles (continued) NR= not reported.

\begin{tabular}{llll}
\hline Author Design & Aim & $\begin{array}{l}\text { Target } \\
\text { group }\end{array}$ & Focus of intervention Main results \\
Year & & &
\end{tabular}

(N)

$\frac{\text { Quality }}{\text { Rigorl }}$

Relevance

\begin{tabular}{|c|c|c|c|c|c|c|}
\hline $\begin{array}{l}\text { Weingarten } \\
\text { et al., } \\
1994^{54}\end{array}$ & $\begin{array}{l}\text { Quasi- } \\
\text { experimental } \\
\text { design }\end{array}$ & $\begin{array}{l}\text { Decrease costs } \\
\text { Improve quality }\end{array}$ & $\begin{array}{l}\text { Physicians } \\
(N=155),\end{array}$ & $\begin{array}{l}\text { Guideline with } \\
\text { recommendation, } \\
\text { reminders, risk } \\
\text { information }\end{array}$ & $\begin{array}{l}\text { Guideline adherence } 50 \% \\
\text { during control periods and } \\
69 \% \text { during intervention } \\
\text { periods }(p<0.001) \text {. Reduced } \\
\text { length of stay }(26 \% p=0.02)\end{array}$ & $+/+$ \\
\hline $\begin{array}{l}\text { Parrino } \\
\text { et al., } \\
198967\end{array}$ & $\begin{array}{l}\text { Quasi- } \\
\text { experimental }\end{array}$ & $\begin{array}{l}\text { Lower drug } \\
\text { utilization } \\
\text { Decrease costs }\end{array}$ & $\begin{array}{l}\text { Physicians } \\
(N=202)\end{array}$ & Cost-feedback & $\begin{array}{l}\text { No significant decline in those } \\
\text { with high antibiotic } \\
\text { expenditures data. No } \\
\text { significant difference between } \\
\text { the feedback and no feedback } \\
\text { group }\end{array}$ & $+/+$ \\
\hline $\begin{array}{l}\text { Polinski } \\
\text { et al., } \\
20 \text { I }^{24}\end{array}$ & $\begin{array}{l}\text { Mixed } \\
\text { methods } \\
\text { (Quasi- } \\
\text { experimental, } \\
\text { interview) }\end{array}$ & $\begin{array}{l}\text { Decrease in cost } \\
\text { of prescribing }\end{array}$ & $\begin{array}{l}\text { Physicians } \\
(n=282)\end{array}$ & $\begin{array}{l}\text { Price information, } \\
\text { evidence to support } \\
\text { recommendation }\end{array}$ & $\begin{array}{l}\text { Increased proportion of } \\
\text { tablet splitting among } \\
\text { participants from } 4.4 \% \\
\text { to } 7.5 \% \text {. Effect varies from } \\
>10 \% \text { increase to }<6 \% \\
\text { decrease }\end{array}$ & $+/+$ \\
\hline $\begin{array}{l}\text { Colbert } \\
\text { et al., } \\
2010^{87}\end{array}$ & $\begin{array}{l}\text { Qualitative } \\
\text { study }\end{array}$ & $\begin{array}{l}\text { Improve } \\
\text { awareness and } \\
\text { responsiveness } \\
\text { to system of } \\
\text { health care }\end{array}$ & $\begin{array}{l}\text { Medical } \\
\text { students } \\
(\mathrm{N}=3 \mathrm{I})\end{array}$ & $\begin{array}{l}\text { Delivering care to } \\
\text { patients who do not } \\
\text { have health insurance }\end{array}$ & $\begin{array}{l}\text { Content analysis identified } \\
\text { six themes: access to } \\
\text { specialists was limited; cost } \\
\text { containment; lack of } \\
\text { resources affects delivery of } \\
\text { care; delays in care due to lack } \\
\text { of insurance; understanding of } \\
\text { larger healthcare system and } \\
\text { free clinic role; and delays in } \\
\text { tests due to language barriers }\end{array}$ & $+/+$ \\
\hline
\end{tabular}




\section{Pursuing High-Value, Cost-Conscious Care}

\section{Discussion}

To ensure sustainable healthcare practices, physicians need to be trained to provide highvalue, cost-conscious care. This realist review was conducted to examine how and under what circumstances educational interventions may help trainees and practicing physicians deliver such care. Three important elements emerged that could inform development of interventions aimed to train physicians: knowledge transmission, reflective practice, and a supportive environment.

First, knowledge transmission appeared to be pertinent to the cost of care and to health economics, to scientific evidence, and to patient preferences. In addition to raising awareness of how physicians contribute to healthcare costs, literature suggested teaching trainees and practicing physicians how to judge medical value and gain insight into patients' personal values. The challenge of teaching physicians to deliver high-value, cost-conscious care seems to be to elicit a general understanding of how their medical decisions relate to value. Second, stimulating reflective practice on these different values through feedback, reflective questions, and group discussions incentivized physicians to think critically about medical decisions. Literature suggested that these elements would be most effective when their training goals are also supported by the environment in which trainees work and learn. Third, such a supportive environment may be necessary for clinical teachers, role models, and other healthcare professionals' trainees to appreciate the importance of high-value, cost-conscious care. These three factors combined provide a framework for the development and further research of educational programs that teach physicians to deliver high-value, cost-conscious care.

The amount of support within an environment may be critical for the success of efforts to train high-value, cost-conscious care. To be successful, transmission of knowledge and reflective practice may work best in environments that feature role models, attention to healthcare teams, and an organization that supports sustainable practice. An unsupportive environment might inadvertently negate the intended training effect, ${ }^{93}$ underlining the importance that trainees witness the delivery of high-value, cost-conscious care in clinical practice. There is a risk of adverse effects of learning from poor role models, and good role models may be scarce, as many physicians who provide clinical education were trained with the assumption that costs have no place in medical decision-making. Adding to confusion is that the current generation of physicians is practicing in a healthcare system that features continuously changing payment systems and accreditation requirements, with a legacy of healthcare costs lacking in transparency, and unstable rules and regulations.

This review should be considered in the light of its limitations. First, due to the variability of definitions given to high-value, cost-conscious care, selection bias cannot be excluded. To 
minimize this risk, no cut-off date was used in the search and all related publications were screened, the oldest articles dating from 1979. Although the field of medicine has evolved tremendously since then, the elements of training inherent in our identified framework and the inherent barriers have remained stable, which reinforces the strength of the findings. A second limitation is the risk of publication bias, which is suggested by apparent effectiveness in $87 \%$ of the studies overall, and in $71 \%$ of the 14 RCTs. We tried to reduce this bias as much as possible by searching multiple databases and placing no restrictions on the quality of journals. Nevertheless, the possibility of publication bias should be considered. Third, the assessment of quality of the included articles, i.e. rigor and relevance, was derived from the professional judgments of the researchers. Although prone to subjectivity, we tried to counteract this through extensive discussions within the research team. Fourth, the factor of supportive environment was partly derived from qualitative data from quantitative and qualitative studies, not as strong as the evidence used to derive knowledge transmission and reflective practice. Quantitative findings using randomized trials demonstrating an influence of the supportive environment on learning outcomes would have provided stronger evidence.

Although the reported effectiveness of educational interventions seems to provide scope for medical education to bring about improvement, training physicians to deliver high-value, cost-conscious care remains a complex task. Further research should focus on what makes a good role model of high-value, cost-conscious care and how such attributes can be cultivated by means of medical education. Additionally, there is a need to investigate how formal education can help mold the culture of the learning environment. Although measuring the value of care is extremely complex, outcome measures that focus solely on volume or costs might promote the incorrect assumption that cheaper is better. Therefore, thoughtful consideration of which outcome measures can be used to evaluate the effectiveness of interventions remains important.

\section{Conclusion}

Research on educating physicians to deliver high-value, cost-conscious care suggests that learning by practicing physicians, resident physicians, and medical students is promoted by combining specific knowledge transmission, reflective practice, and a supportive environment. These factors should be considered when educational interventions are being developed. 


\section{Pursuing High-Value, Cost-Conscious Care}

\section{References}

1. Reinhardt UE. Waste vs. Value in American Health Care. Economix 2013; http://economix.blogs. nytimes.com/2013/09/13/waste-vs-value-in-american-health-care/?_r=0. Accessed january 2014, 2014.

2. Berwick DM, Hackbarth AD. Eliminating waste in US health care. JAMA : the journal of the American Medical Association. 2012;307(14):1513-1516.

3. Blumenthal D. Controlling health care expenditures. The New England journal of medicine. 2001;344(10):766-769.

4. Altarum. Insights from Monthly National Health Spending data through Juli 2015. 2015. http:// webcache.googleusercontent.com/search?q=cache:_Lhcwh6fS8MJ:altarum.org/sites/default/files/ uploaded-related-files/CSHS-Spending-Brief_February_2015.pdf $+\& c d=1 \& h l=n l \& c t=c l n k \& g l=n l$. Accessed 13 september 2015.

5. Duke P. The ABIM foundation's Choosing wisely communication module. [webpage]. 2013; http:// modules.choosingwisely.org/modules/m_00/. Accessed February, 2014.

6. (ACP) ACop, (AAIM) AfAIM. High Value Care Curriculum. 2012, 2014.

7. Owens DK, Qaseem A, Chou R, Shekelle P, Clinical Guidelines Committee of the American College of P. High-value, cost-conscious health care: concepts for clinicians to evaluate the benefits, harms, and costs of medical interventions. Annals of internal medicine. 2011;154(3):174-180.

8. OECD. Health spending Total, \% of GDP, 1970 - 2013. Source: OECD Health Data: Health expenditure and financing: Health expenditure indicators 2013; https://data.oecd.org/chart/4f22. Accessed February 2015, 2015.

9. Cutler D. The Value Equation in Health Care Paper presented at: National Health Care Reform: Policy Options and Imperatives2007; Rice University, Houston, USA..

10. Wong G, Greenhalgh T, Westhorp G, Buckingham J, Pawson R. RAMESES publication standards: realist syntheses. BMC medicine. 2013;11:21.

11. Collaboration B. BEME data coding sheet. BEME 2014; http://bemecollaboration.org/. Accessed june 2014, 2014.

12. Cochrane TC. Data collection form for RCT and non-RCT. 2014; http://www.cochranelibrary.com/ cochrane-database-of-systematic-reviews/supplements.html. Accessed June 2014, 2014.

13. Pawson R, Greenhalgh T, Harvey G, Walshe K. Realist synthesis: an introduction. Manchester: ESRC Research Methods Programme, University of Manchester. 2004.

14. Sussman AJ, Chabra B, Coblyn J, et al. Helping primary care physicians lower pharmaceutical expenses: An academic medical center's experience. Journal of Clinical Outcomes Management. 2004;11(5):290-295.

15. Spiegel CT, et al. Modification of Decision-Making Behavior of Third-Year Medical Students. Journal of medical education. 1982;57(10):t1769-1777.

16. Post J, Reed D, Halvorsen AJ, Huddleston J, McDonald F. Teaching high-value, cost-conscious care: improving residents' knowledge and attitudes. The American journal of medicine. 2013;126(9):838-842.

17. Chandawarkar RY, Taylor S, Abrams P, et al. Cost-aware care: critical core competency. Archives of surgery (Chicago, Ill. : 1960). 2007;142(3):222-226.

18. Ellemdin S, Rheeder P, Soma P. Providing clinicians with information on laboratory test costs leads to reduction in hospital expenditure. South African medical journal = Suid-Afrikaanse tydskrifvir geneeskunde. 2011;101(10):746-748.

19. Davidoff F, Goodspeed R, Clive J. Changing test ordering behavior. A randomized controlled trial comparing probabilistic reasoning with cost-containment education. Medical care. 1989;27(1):45-58. 
20. Englander R, Agostinucci W, Zalneraiti E, Carraccio CL. Teaching residents systems-based practice through a hospital cost-reduction program: a "win-win" situation. Teaching and learning in medicine. 2006;18(2):150-152.

21. Stuebing EA, Miner TJ. Surgical vampires and rising health care expenditure: reducing the cost of daily phlebotomy. Archives of surgery (Chicago, Ill. : 1960). 2011;146(5):524-527.

22. Sommers BD, Desai N, Fiskio J, et al. An educational intervention to improve cost-effective care among medicine housestaff: a randomized controlled trial. Academic medicine : journal of the Association of American Medical Colleges. 2012;87(6):719-728.

23. Hart J, Salman H, Bergman M, et al. Do drug costs affect physicians' prescription decisions? Journal of internal medicine. 1997;241(5):415-420.

24. Polinski JM, Schneeweiss S, Maclure M, Marshall B, Ramsden S, Dormuth C. Time series evaluation of an intervention to increase statin tablet splitting by general practitioners. Clinical therapeutics. 2011;33(2):235-243.

25. Willens HJ, Nelson K, Hendel RC. Appropriate use criteria for stress echocardiography: impact of updated criteria on appropriateness ratings, correlation with pre-authorization guidelines, and effect of temporal trends and an educational initiative on utilization. JACC. Cardiovascular imaging. 2013;6(3):297-309.

26. Qureshi NA, Neyaz Y, Khoja T, Magzoub MA, Haycox A, Walley T. Effectiveness of three interventions on primary care physicians' medication prescribing in Riyadh City, Saudi Arabia. Eastern Mediterranean health journal $=$ La revue de sante de la Mediterranee orientale $=$ al-Majallah al-sihhiyah li-sharq al-mutawassit. 2011;17(2):172-179.

27. Roth EJ, Plastaras CT, Mullin MS, Fillmore J, Moses ML. A simple institutional educational intervention to decrease use of selected expensive medications. Archives of physical medicine and rehabilitation. 2001;82(5):633-636.

28. Schroeder SA, Myers LP, McPhee SJ, et al. The failure of physician education as a cost containment strategy. Report of a prospective controlled trial at a university hospital. JAMA: the journal of the American Medical Association. 1984;252(2):225-230.

29. Gitelis M, Vigneswaran Y, Ujiki MB, et al. Educating surgeons on intraoperative disposable supply costs during laparoscopic cholecystectomy: a regional health system's experience. American journal of surgery. 2015;209(3):488-492.

30. Vigneswaran Y, Linn JG, Gitelis M, et al. Educating surgeons may allow for reduced intraoperative costs for inguinal herniorrhaphy. Journal of the American College of Surgeons. 2015;220(6):1107-1112.

31. Das AK, Rahman MS. Prescribing vitamins at primary health care level: Exploration of facts, factors and solution. Bangladesh Journal of Pharmacology. 2010;5(2):92-97.

32. de Leon N, Sharpton S, Burg C, et al. The development and implementation of a bundled quality improvement initiative to reduce inappropriate stress ulcer prophylaxis. ICU Director. 2013;4(6):322-325.

33. Whiteside ME, Lefkowitz S, Justiniani FR, Ratzan K. Changing prescribing patterns: a program of physician education. Hospital formulary. 1987;22(6):561-563, 566, 568.

34. Cammisa C, Partridge G, Ardans C, Buehrer K, Chapman B, Beckman H. Engaging physicians in change: results of a safety net quality improvement program to reduce overuse. American journal of medical quality: the official journal of the American College of Medical Quality. 2011;26(1):26-33.

35. SelfTH, Smith SL, Boswell RL, Miller WA. Medical education provided by a clinical pharmacist: impact on the use and cost of corticosteroid therapy in chronic obstructive pulmonary disease. Drug intelligence \& clinical pharmacy. 1984;18(3):241-244. 


\section{Pursuing High-Value, Cost-Conscious Care}

36. McKay RM, Vrbova L, Fuertes E, et al. Evaluation of the do bugs need drugs? Program in British Columbia: Can we curb antibiotic prescribing? Canadian Journal of Infectious Diseases and Medical Microbiology. 2011;22(1):19-24.

37. Barbarello-Andrews L, Susla G, Ng V, St. John D, Lau C. Cost-effective medication use in critical care: Capital health system's experience in VHA's MUSIC Program. Journal of Clinical Outcomes Management. 2006;13(11):615-622.

38. Marconi GP, Nager AL. Teaching residents established guidelines and standards of care to strengthen their cost-containment practices. Managed care (Langhorne, Pa.). 2010;19(5):46-51.

39. Blackstone ME, Miller RS, Hodgson AJ, Cooper SS, Blackhurst DW, Stein MA. Lowering hospital charges in the trauma intensive care unit while maintaining quality of care by increasing resident and attending physician awareness. The Journal of trauma. 1995;39(6):1041-1044.

40. Elligsen M, Walker SA, Pinto R, et al. Audit and feedback to reduce broad-spectrum antibiotic use among intensive care unit patients: a controlled interrupted time series analysis. Infection control and hospital epidemiology : the offcial journal of the Society of Hospital Epidemiologists of America. 2012;33(4):354-361.

41. Dowling PT, et al. An Education Program to Reduce Unnecessary Laboratory Tests by Residents. Academic Medicine. 1989;64(7):410-412.

42. Fakih MG, Pena ME, Shemes $S$, et al. Effect of establishing guidelines on appropriate urinary catheter placement. Academic emergency medicine : official journal of the Society for Academic Emergency Medicine. 2010;17(3):337-340.

43. Verstappen WH, van der Weijden T, Sijbrandij J, et al. Effect of a practice-based strategy on test ordering performance of primary care physicians: a randomized trial. JAMA: the journal of the American Medical Association. 2003;289(18):2407-2412.

44. Poppleton VK, Moynihan PJ, Hickey PA. Clinical practice guidelines: The Boston experience. Progress in Pediatric Cardiology. 2003;18(1):75-83.

45. Sleath B, Collins T, Kelly HW, McCament-Mann L, Lien T. Effect of including both physicians and pharmacists in an asthma drug-use review intervention. American journal of health-system pharmacy: AJHP : official journal of the American Society of Health-System Pharmacists. 1997;54(19):2197-2200.

46. Ijo I, Feyerharm J. Pharmacy intervention on antimicrobial management of critically ill patients. Pharmacy Practice. 2011;9(2):106-109.

47. Bernal-Delgado E, Galeote-Mayor M, Pradas-Arnal F, Peiro-Moreno S. Evidence based educational outreach visits: effects on prescriptions of non-steroidal anti-inflammatory drugs. Journal of epidemiology and community health. 2002;56(9):653-658.

48. Bhatia RS, Milford CE, Picard MH, Weiner RB. An educational intervention reduces the rate of inappropriate echocardiograms on an inpatient medical service. JACC. Cardiovascular imaging. 2013;6(5):545-555.

49. Hux JE, Melady MP, DeBoer D. Confidential prescriber feedback and education to improve antibiotic use in primary care: a controlled trial. CMAJ: Canadian Medical Association journal = journal de l'Association medicale canadienne. 1999;161(4):388-392.

50. Larmour I, Pignataro S, Barned KL, Mantas S, Korman MG. A therapeutic equivalence program: evidence-based promotion of more efficient use of medicines. The Medical journal of Australia. 2011;194(12):631-634.

51. Phillips L, Landsberg KF. Evaluations of a newsletter in altering physicians' prescribing patterns. Canadian Journal of Hospital Pharmacy. 1986;39(4):102-104.

52. Ziskind AA, Portelli J, Rodriguez S, et al. Successful use of education and cost-based feedback 
strategies to reduce physician utilization of low-osmolality contrast agents in the cardiac catheterization laboratory. The American journal of cardiology. 1994;73(16):1219-1221.

53. Shane R, Nishimura L. Strategic management of therapeutic advances: Experience with colonystimulating factors. Hospital Pharmacy. 1994;29(9):824+826-829.

54. Weingarten SR, Riedinger MS, Conner L, et al. Practice guidelines and reminders to reduce duration of hospital stay for patients with chest pain. An interventional trial. Annals of internal medicine. 1994;120(4):257-263.

55. Sucov A, Bazarian JJ, deLahunta EA, Spillane L. Test ordering guidelines can alter ordering patterns in an academic emergency department. The Journal of emergency medicine. 1999;17(3):391-397.

56. Okpara AU, Maswoswe JJ, Stewart K. Criteria-based antimicrobial IV to oral conversion program. Formulary. 1995;30(6):343-348.

57. Legare F, Labrecque M, LeBlanc A, et al. Training family physicians in shared decision making for the use of antibiotics for acute respiratory infections: a pilot clustered randomized controlled trial. Health expectations: an international journal of public participation in health care and health policy. 2010;14 Suppl 1:96-110.

58. Legare F, Labrecque M, Cauchon M, Castel J, Turcotte S, Grimshaw J. Training family physicians in shared decision-making to reduce the overuse of antibiotics in acute respiratory infections: a cluster randomized trial. CMAJ: Canadian Medical Association journal=journal de l'Association medicale canadienne. 2012;184(13):E726-734.

59. Braido F, Comaschi M, Valle I, et al. Knowledge and health care resource allocation: CME/CPD course guidelines-based efficacy. European annals of allergy and clinical immunology. 2012;44(5):193-199.

60. James E, Cyriac J. Impact of educational interventions on the physicians for early switchover of parenteral drugs to oral therapy. European Journal Hospital Pharmacy. 2014;22:176-178.

61. Lee TC, Frenette C, Jayaraman D, Green L, Pilote L. Antibiotic self-stewardship: trainee-led structured antibiotic time-outs to improve antimicrobial use. Annals of internal medicine. 2014;161(10 Suppl):S53-58.

62. Yang Z, Zhao P, Wang J, et al. DRUGS system enhancing adherence of Chinese surgeons to antibiotic use guidelines during perioperative period. PloS one. 2014;9(8):e102226.

63. Fortuna RJ, Zhang F, Ross-Degnan D, et al. Reducing the prescribing of heavily marketed medications: a randomized controlled trial. Journal of general internal medicine. 2009;24(8):897-903.

64. Wein PJ, Hoffman RP. Promoting the cost-effective utilization of cefoxitin with a drug use education program. Hospital formulary. 1987;22(3):299-306.

65. Gregory KD, Hackmeyer P, Gold L, Johnson AI, Platt LD. Using the continuous quality improvement process to safely lower the cesarean section rate. The Joint Commission journal on quality improvement. 1999;25(12):619-629.

66. Pasquale TR, Komorny KM, Letting-Mangira D, Peshek S. A Pharmacist-Physician Antibiotic Support Team. Pharmacy \& Therapeutics. 2004;29(1):33-40.

67. Parrino TA. The nonvalue of retrospective peer comparison feedback in containing hospital antibiotic costs. The American journal of medicine. 1989;86(4):442-448.

68. McPhee SJ, et al. Lessons for Teaching Cost Containment. Journal of medical education. 1984;59(9):722-729.

69. von Ferber L, Bausch J, Koster I, Schubert I, Ihle P. Pharmacotherapeutic circles. Results of an 18-month peer-review prescribing-improvement programme for general practitioners.

PharmacoEconomics. 1999;16(3):273-283. 


\section{Pursuing High-Value, Cost-Conscious Care}

70. Miyakis S, Karamanof G, Liontos M, Mountokalakis TD. Factors contributing to inappropriate ordering of tests in an academic medical department and the effect of an educational feedback strategy. Postgraduate medical journal. 2006;82(974):823-829.

71. Moriates C, Soni K, Lai A, Ranji S. The value in the evidence: teaching residents to "choose wisely". JAMA internal medicine. 2013;173(4):308-310.

72. Attali M, Barel Y, Somin M, et al. A cost-effective method for reducing the volume of laboratory tests in a university-associated teaching hospital. The Mount Sinai journal of medicine, New York. 2006;73(5):787-794.

73. Bates DW, Kuperman GJ, Rittenberg E, et al. A randomized trial of a computer-based intervention to reduce utilization of redundant laboratory tests. The American journal of medicine. 1999;106(2):144-150.

74. Thakkar RN, Kim D, Knight AM, Riedel S, Vaidya D, Wright SM. Impact of an educational intervention on the frequency of daily blood test orders for hospitalized patients. American journal of clinical pathology. 2015;143(3):393-397.

75. Zimmerman DR, Collins TM, Lipowski EE, Sainfort F. Evaluation of a DUR intervention: a case study of histamine antagonists. Inquiry : a journal of medical care organization, provision and financing. 1994;31(1):89-101.

76. Zunker RJ, Carlson DL. Economics of using pharmacists as advisers to physicians in risk-sharing contracts. American journal of health-system pharmacy: AJHP : official journal of the American Society of Health-System Pharmacists. 2000;57(8):753-755.

77. Sicotte C, Pineault R, Tilquin C, Contandriopoulos AP. The diluting effect of medical work groups on feedback efficacy in changing physician's practice. Journal of behavioral medicine. 1996;19(4):367-383.

78. Krinsley JS. Test-ordering strategy in the intensive care unit. Journal of intensive care medicine. 2003;18(6):330-339.

79. Rudy DW, Ramsbottom-Lucier M, Griffith CH, 3rd, Georgesen JC, Wilson JF. A pilot study assessing the influences of charge data and group process on diagnostic test ordering by residents. Academic medicine : journal of the Association of American Medical Colleges. 2001;76(6):635-637.

80. Rotman BL, Sullivan AN, McDonald TW, et al. A randomized controlled trial of a computer-based physician workstation in an outpatient setting: implementation barriers to outcome evaluation. Journal of the American Medical Informatics Association: JAMIA. 1996;3(5):340-348.

81. Bornard L, Dellamonica J, Hyvernat H, et al. Impact of an assisted reassessment of antibiotic therapies on the quality of prescriptions in an intensive care unit. Medecine et maladies infectieuses. 2011;41(9):480-485.

82. Manheim LM, Feinglass J, Hughes R, Martin GJ, Conrad K, Hughes EF. Training house officers to be cost conscious. Effects of an educational intervention on charges and length of stay. Medical care. 1990;28(1):29-42.

83. Niquille A, Ruggli M, Buchmann M, Jordan D, Bugnon O. The nine-year sustained costcontainment impact of swiss pilot physicians-pharmacists quality circles. The Annals of pharmacotherapy. 2010;44(4):650-657.

84. Zeleznik C, Gonnella JS. Jefferson Medical College Student Model Utilization Review Committee. Academic Medicine. 1979;54(11):848-851.

85. Gist D, Llorente J, Mayer J. A clinical algorithm for the management of abnormal mammograms. A community hospital's experience. The Western journal of medicine. 1997;166(1):21-28.

86. Lyle CB, Jr., et al. Teaching Cost Containment to House Officers at Charlotte Memorial Hospital. Academic Medicine. 1979;54(11):856-862. 
87. Colbert CY, Ogden PE, Lowe D, Moffitt MJ. Students learn systems-based care and facilitate system change as stakeholders in a free clinic experience. Advances in health sciences education: theory and practice. 2010;15(4):533-545.

88. Mallows JL. The effect of a gold coin fine on C-reactive protein test ordering in a tertiary referral emergency department. The Medical journal of Australia. 2013;199(11):813-814.

89. Collins TM, Mott DA, Bigelow WE, Zimmerman DR. A controlled letter intervention to change prescribing behavior: results of a dual-targeted approach. Health services research. 1997;32(4):471-489.

90. Ferris TG, Shea T, Jacobson BC, et al. A population-based intervention to improve management of patients on chronic acid suppression: A group-randomized trial. Journal of Clinical Outcomes Management. 2005;12(1):35-43.

91. Landgren FT, Harvey KJ, Mashford ML, Moulds RF, Guthrie B, Hemming M. Changing antibiotic prescribing by educational marketing. The Medical journal of Australia. 1988;149(11-12):595-599.

92. Smith SR. An Evaluation of a Computerized Exercise in Teaching Cost Consciousness. Journal of medicaleducation. 1983;58(2):146-148.

93. Glicken AD, Merenstein GB. Addressing the hidden curriculum: understanding educator professionalism. Medical teacher. 2007;29(1):54-57. 


\section{Appendix 2.I: Eligibility form}

\section{General Information}

\begin{tabular}{|l|l|}
\hline Endnote ID number & \\
\hline Source database & $\begin{array}{l}\text { PubMed } \\
\text { ERIC } \\
\text { EMBASE } \\
\text { Cochrane }\end{array}$ \\
\hline Date form completed (dd/mm/yyy) & AOP,IV, LAS \\
\hline Name/ID of person extracting data & \\
\hline $\begin{array}{l}\text { Report title } \\
\text { (title of paper/ abstract/ report that data are } \\
\text { extracted from) }\end{array}$ & \\
\hline Author (first and second) & \\
\hline Year of publication & \\
\hline Continent & \\
\hline Country & \\
\hline Journal & \\
\hline
\end{tabular}

\section{Eligibility}

\begin{tabular}{|l|l|}
\hline Does this article describe an intervention? & $\begin{array}{l}\text { I= yes } \\
0=\text { no }\end{array}$ \\
\hline Does this intervention contain educational elements? & $\begin{array}{l}\mathrm{I}=\text { yes } \\
0=\text { no }\end{array}$ \\
\hline Does this article evaluate the intervention? & $\begin{array}{l}\mathrm{I}=\text { yes } \\
0=\text { no }\end{array}$ \\
\hline $\begin{array}{l}\text { Does the target population contain physicians, residents or medical students? } \\
\text { I= yes } \\
0=\text { no }\end{array}$ \\
\hline $\begin{array}{l}\text { Is the aim of the study to I) reduce volume of health care services, 2) reduce health } \\
\text { care expenditures, } 3 \text { ) improve quality of care, 4) improve knowledge and/or attitude } \\
\text { regarding cost of care? }\end{array}$ & $\begin{array}{l}\text { I= no } \\
0=\end{array}$ \\
\hline $\begin{array}{l}\text { Inclusion if total of points= } 5 \text { Exclusion if total of points } \leq 4 \\
\square \text { INCLUDE } \square \text { EXCLUDE }\end{array}$ & Total of points \\
\hline Reason for exclusion & \\
\hline
\end{tabular}




\section{Design}

\begin{tabular}{|c|c|}
\hline Type of study & $\begin{array}{l}\text { I. Review } \\
\text { Literature review } \\
\text { Narrative review } \\
\text { Systematic review } \\
\text { Realist review } \\
\text { Other.... } \\
\text { 2. Experimental design } \\
\text { RCT } \\
\text { Controlled Clinical Trial } \\
\text { Quasi-randomized Trial } \\
\text { Cross over series } \\
\text { Other... } \\
\text { 3. Quasi-experimental design } \\
\text { Time Series } \\
\text { Repeated measures } \\
\text { Non-equivalent control group } \\
\text { Other... } \\
\text { 4. Observational studies } \\
\text { 5. Qualitative studies } \\
\text { 6. Mixed methods } \\
\text { 7. (Controlled) Before and After Study } \\
\text { 9. Other design (specify): }\end{array}$ \\
\hline $\begin{array}{l}\text { Describe target } \\
\text { population }\end{array}$ & $\begin{array}{l}\text { Medical Students } \\
\text { Residents } \\
\text { Physicians } \\
\text { Nurses } \\
\text { Patients } \\
\text { Combination of above. }\end{array}$ \\
\hline
\end{tabular}




\begin{tabular}{|c|c|}
\hline Setting & $\begin{array}{l}\text { Hospital } \\
\text { Medical school } \\
\text { Primary care } \\
\text { Private Care } \\
\text { Other, room for explanatory note. }\end{array}$ \\
\hline $\begin{array}{l}\text { Specialty or } \\
\text { discipline }\end{array}$ & Open question. \\
\hline $\begin{array}{l}\text { Intervention } \\
\text { category }\end{array}$ & $\begin{array}{l}\text { Educational intervention } \\
\text { Organizational intervention (without information about aim intervention to } \\
\text { target population) } \\
\text { Organizational intervention with educational elements. } \\
\text { Organizational (information provided: Not specified) } \\
\text { Other, open question. }\end{array}$ \\
\hline $\begin{array}{l}\text { Main outcome } \\
\text { measure }\end{array}$ & $\begin{array}{l}\text { Reaction } \\
\text { Attitude } \\
\text { Knowledge } \\
\text { Costs } \\
\text { Behavior } \\
\text { Volume/utilization }\end{array}$ \\
\hline
\end{tabular}

DO NOT PROCEED IF STUDY EXCLUDED FROM REVIEW (2. Eligibility) 


\section{Appendix 2.2: Abstraction form}

\section{Methods}

\begin{tabular}{|l|l|l|}
\hline & Description & $99=$ unknown \\
\hline Inclusion criteria of participants & & \\
\hline Exclusion criteria of participants & & \\
\hline Method/s of recruitment of participants & & \\
\hline Total number of subjects & & \\
\hline
\end{tabular}

\begin{tabular}{|l|l|l|}
\hline & Descriptions as stated in report/paper & \multicolumn{2}{|l|}{$99=$ unknown } \\
\hline Quoted Aim of study & \multicolumn{2}{|l|}{} \\
\hline Perceived aim of study & & \multicolumn{2}{|l|}{ I = yes } \\
\hline $\begin{array}{l}\text { Tied to theoretical/conceptional } \\
\text { framework }\end{array}$ & \\
\hline Name of theory & & \\
\hline Start date study & & \\
\hline End date study & & \\
\hline Duration of exposure/participation & & \\
\hline $\begin{array}{l}\text { Duration of study incl. follow-up or } \\
\text { evaluation }\end{array}$ & & \\
\hline Pre-intervention period & & \\
\hline Follow-up period & & \\
\hline
\end{tabular}




\section{Participants}

Provide overall data and, if available, comparative data for each intervention or comparison group.

\begin{tabular}{|l|l|l|}
\hline & Description as stated in report/paper & 99=unknown. \\
\hline $\begin{array}{l}\text { Number of participants in group I } \\
\text { (intervention group) }\end{array}$ & & \\
\hline $\begin{array}{l}\text { Numbers of participants in group 2 } \\
\text { (control group) }\end{array}$ & & \\
\hline Numbers of participants in group 3 & & \\
\hline Numbers of participants in group 4 & & \\
\hline Baseline imbalances & & \\
\hline $\begin{array}{l}\text { Subgroups measured } \\
\text { If yes, on which characteristics? }\end{array}$ & & \\
\hline Subgroups reported & & \\
\hline
\end{tabular}

\section{Intervention group}

\begin{tabular}{|l|l|l|}
\hline & Description as stated in report/paper & $\begin{array}{l}\text { I= yes } \\
0=\text { no }\end{array}$ \\
\hline Name intervention & $y / n$ \\
\hline Course & $y / n$ \\
\hline Program & $y / n$ \\
\hline Curriculum & $y / n$ \\
\hline Project & Open question. \\
\hline Other &
\end{tabular}




\begin{tabular}{|c|c|}
\hline & Intervention I \\
\hline $\begin{array}{l}\text { Program type } \\
\text { (combinations are possible) }\end{array}$ & $\begin{array}{l}\text { 1. Workshop } \\
\text { 2. Short course } \\
\text { 3. Seminar Series } \\
\text { 4. Longitudinal Program } \\
\text { 5. Fellowship } \\
\text { 6. Master's program } \\
\text { 7. Certificate/ diploma course } \\
\text { 8. Computer-based program } \\
\text { 9. Mentorship program } \\
\text { 10. Other (please specify) }\end{array}$ \\
\hline Program type specified & Open question. \\
\hline $\begin{array}{l}\text { Instructional methods (combinations } \\
\text { are possible) }\end{array}$ & $\begin{array}{l}\text { Needs assessment } \\
\text { Didactic teaching (e.g. lecture) } \\
\text { Small group discussion } \\
\text { Case-based teaching } \\
\text { Problem-based learning } \\
\text { Experiential learning (i.e. learning through practice opportunities) } \\
\text { Micro-teaching (e.g. Practical teaching experience with immediate feedback) } \\
\text { Interactive exercises } \\
\text { Role plays and simulations } \\
\text { Films, videotapes and audiotapes } \\
\text { Independent learning / projects } \\
\text { Written materials and readings } \\
\text { Computer-based materials } \\
\text { Coaching } \\
\text { Utilization feedback } \\
\text { Other } \\
\text { Peer feedback } \\
\text { Academic detailing }\end{array}$ \\
\hline Instructional methods specified & Open question. \\
\hline \multicolumn{2}{|l|}{ Frequency } \\
\hline Length & \\
\hline
\end{tabular}

If multiple interventions were compared, please fill in a new table. 


\section{Pursuing High-Value, Cost-Conscious Care}

\begin{tabular}{|c|c|}
\hline & Intervention 2 \\
\hline Program type & $\begin{array}{l}\text { 1. Workshop } \\
\text { 2. Short course } \\
\text { 3. Seminar Series } \\
\text { 4. Longitudinal Program } \\
\text { 5. Fellowship } \\
\text { 6. Master's program } \\
\text { 7. Certificate/ diploma course } \\
\text { 8. Computer-based program } \\
\text { 9. Mentorship program } \\
\text { 10. Other (please specify) }\end{array}$ \\
\hline \multicolumn{2}{|l|}{ Program type specified } \\
\hline $\begin{array}{l}\text { Instructional methods } \\
\text { (Possibly more than I) }\end{array}$ & $\begin{array}{l}\text { Needs assessment } \\
\text { Didactic teaching (e.g. lecture) } \\
\text { Small group discussion } \\
\text { Case-based teaching } \\
\text { Problem-based learning } \\
\text { Experiential learning (i.e. learning through practice opportunities) } \\
\text { Micro-teaching (e.g. Practical teaching experience with immediate feedback) } \\
\text { Interactive exercises } \\
\text { Role plays and simulations } \\
\text { Films, videotapes and audiotapes } \\
\text { Independent learning / projects } \\
\text { Written materials and readings } \\
\text { Computer-based materials } \\
\text { Coaching } \\
\text { Utilization feedback } \\
\text { Other } \\
\text { Peer feedback } \\
\text { Academic detailing }\end{array}$ \\
\hline \multicolumn{2}{|l|}{ Instructional methods specified } \\
\hline \multicolumn{2}{|l|}{ Frequency } \\
\hline Length & \\
\hline
\end{tabular}




\section{Control group}

\begin{tabular}{|c|c|c|}
\hline $\begin{array}{l}\text { Name of intervention } \\
\text { in case of absence of intervention } \\
\text { write: no intervention. }\end{array}$ & & \\
\hline Design & & $\begin{array}{l}1=\text { yes } \\
0=\text { no }\end{array}$ \\
\hline \multicolumn{3}{|l|}{ Within-design } \\
\hline \multicolumn{3}{|l|}{ Between-design } \\
\hline \multicolumn{3}{|l|}{ Describe control group } \\
\hline Program type & $\begin{array}{l}\text { 1. Workshop } \\
\text { 2. Short course } \\
\text { 3. Seminar Series } \\
\text { 4. Longitudinal Program } \\
\text { 5. Fellowship } \\
\text { 6. Master's program } \\
\text { 7. Certificate/ diploma course } \\
\text { 8. Computer-based program } \\
\text { 9. Mentorship program } \\
\text { 10. Other (please specify) }\end{array}$ & \\
\hline \multicolumn{3}{|l|}{ Program type specified } \\
\hline \multicolumn{3}{|l|}{$\begin{array}{l}\text { Instructional methods } \\
\text { (Possibly more than I) }\end{array}$} \\
\hline \multicolumn{3}{|l|}{ Instructional methods specified } \\
\hline \multicolumn{3}{|l|}{ Frequency } \\
\hline Length & & \\
\hline
\end{tabular}




\section{Outcomes}

Main Outcome I
\begin{tabular}{|l|l|l|}
\hline & Description as stated in report/paper \\
\hline Main outcome measure & $\begin{array}{l}\text { Reaction } \\
\text { Attitude } \\
\text { Knowledge } \\
\text { Costs } \\
\text { Behavior } \\
\text { Volume/utilization }\end{array}$ \\
\hline Main outcome described (if possible, \\
provide definition) & & \\
\hline Result & & \\
\hline
\end{tabular}

\section{Main outcome 2}

$\left.\begin{array}{|l|l|l|}\hline & \text { Description as stated in report/paper } \\ \hline \text { Main outcome measure } & \begin{array}{l}\text { Reaction } \\ \text { Attitude } \\ \text { Knowledge } \\ \text { Costs } \\ \text { Behavior } \\ \text { Volume/utilization }\end{array} \\ \hline \text { Main outcome described (if possible, } \\ \text { provide definition) }\end{array}\right)$




\section{Main outcome 3}

\begin{tabular}{|l|l|l|}
\hline & Description as stated in report/paper \\
\hline Main outcome measure & $\begin{array}{l}\text { Reaction } \\
\text { Attitude } \\
\text { Knowledge } \\
\text { Costs } \\
\text { Behavior } \\
\text { Volume/utilization }\end{array}$ \\
\hline $\begin{array}{l}\text { Main outcome described (if possible, } \\
\text { provide definition) }\end{array}$ & & \\
\hline Result & & \multicolumn{2}{|l|}{} \\
\hline
\end{tabular}

\section{Outcomes}

Copy and paste table for each outcome.

\section{Outcome}

\begin{tabular}{|l|l|l|}
\hline & Description as stated in report/paper & $\begin{array}{l}\text { Location in text } \\
\text { (p. \& I/fig/table) }\end{array}$ \\
\hline $\begin{array}{l}\text { Time points measured } \\
\text { (specify whether from start or end of } \\
\text { intervention) }\end{array}$ & & \\
\hline Time points reported & & \\
\hline $\begin{array}{l}\text { Unit of measurement } \\
\text { (if relevant) }\end{array}$ & & \\
\hline Kirk Patrick level & $\mathrm{I}$ & \\
& $2 \mathrm{a}$ & \\
& $2 \mathrm{~b}$ & \\
\hline KP described & 3 & \\
\hline Main conclusion & $4 \mathrm{a}$ & \\
\hline & $4 \mathrm{~b}$ & \\
\hline
\end{tabular}




\section{Applicability}

\begin{tabular}{|c|c|c|c|}
\hline $\begin{array}{l}\text { Does the study answer the research } \\
\text { question stated in the article? }\end{array}$ & & $\begin{array}{l}\square \\
\text { No }\end{array}$ & $\begin{array}{l}\square \\
\text { Unclear }\end{array}$ \\
\hline Relevance & 口 & $\square$ & $\square$ \\
\hline $\begin{array}{l}\text { Does the study address the review } \\
\text { question? }\end{array}$ & Yes & No & Unclear \\
\hline
\end{tabular}

\section{Quality}

\begin{tabular}{|c|c|c|}
\hline Key conclusions of study authors & & 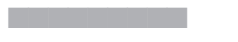 \\
\hline Important references & & 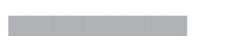 \\
\hline \multirow[t]{2}{*}{$\begin{array}{l}\text { Rigor } \\
\text { Study quality }\end{array}$} & \multicolumn{2}{|l|}{$\begin{array}{l}\text { Rate overall quality. } \\
\text { Low High } \\
\text { Quality Quality } \\
<\longrightarrow\end{array}$} \\
\hline & $\begin{array}{l}\text { I No clear conclusions can be drawn. Not significant. } \\
2 \text { Results weak/ambiguous, but there appears to be at } \\
3 \text { Conclusions can probably be based on the results. } \\
4 \text { Results are clear and very likely to be true. } \\
5 \text { Results are unequivocal. }\end{array}$ & \\
\hline $\begin{array}{l}\text { Rigor } \\
\text { Strengths and weaknesses }\end{array}$ & \multicolumn{2}{|c|}{$\begin{array}{l}\text { Please describe strengths and weaknesses of the study design, evaluation } \\
\text { methods, study implementation and data analysis. }\end{array}$} \\
\hline \multicolumn{3}{|l|}{$\begin{array}{l}\text { Rigor } \\
\text { Avenues for further research }\end{array}$} \\
\hline \multicolumn{3}{|l|}{$\begin{array}{l}\text { Rigor } \\
\text { New "Insights"/Implications for } \\
\text { cost-education }\end{array}$} \\
\hline \multicolumn{3}{|l|}{$\begin{array}{l}\text { Relevance } \\
\text { Based on this article, does "cost inter- } \\
\text { vention" make a difference? }\end{array}$} \\
\hline $\begin{array}{l}\text { Did the intervention cause } \\
\text { permanent changes? }\end{array}$ & & \\
\hline
\end{tabular}




\section{Appendix 2.3: Descriptive overview of included articles}

\begin{tabular}{|c|c|c|c|c|c|c|c|c|}
\hline & Aim & & & & & & tegc & \\
\hline Year & & Shecialty & intervention & $k$ & leasurement & $\mathrm{V}$ & $\$$ & \\
\hline $\begin{array}{l}\text { Attali } \\
\text { et al., } \\
2006\end{array}$ & $\begin{array}{l}\downarrow \text { Inappropriate } \\
\text { laboratory } \\
\text { testing }\end{array}$ & $\begin{array}{l}\text { Residents, physicians } \\
\text { Internal medicine }\end{array}$ & $\begin{array}{l}\text { Lecture on } \\
\text { excessive or } \\
\text { inappropriate } \\
\text { ordering of tests, } \\
\text { supervised ordering }\end{array}$ & $K+R$ & $\begin{array}{l}\text { Volume, costs of } \\
\text { orders }\end{array}$ & $x$ & $x$ & $x$ \\
\hline $\begin{array}{l}\text { Barbarello } \\
\text { et al., } \\
2006\end{array}$ & $\begin{array}{l}\uparrow \text { Efficiency } \\
\uparrow \text { cost } \\
\text { effectiveness }\end{array}$ & $\begin{array}{l}\text { Physicians, other } \\
\text { Critical care unit }\end{array}$ & $\begin{array}{l}\text { Educational } \\
\text { communication } \\
\text { forms and posters, } \\
\text { patient-specific } \\
\text { physician- } \\
\text { pharmacists } \\
\text { discussion }\end{array}$ & $K+R+S$ & $\begin{array}{l}\text { Volume, costs, } \\
\text { appropriateness* } \\
\text { of drugs and } \\
\text { admission }\end{array}$ & $x$ & $x$ & $\mathrm{x}$ \\
\hline $\begin{array}{l}\text { Bates } \\
\text { et al., } \\
1999\end{array}$ & $\begin{array}{l}\downarrow \text { Redundant } \\
\text { test ordering }\end{array}$ & $\begin{array}{l}\text { Physicians } \\
\text { Unknown }\end{array}$ & $\begin{array}{l}\text { Feedback-alert for } \\
\text { redundant tests }\end{array}$ & $\mathrm{R}$ & $\begin{array}{l}\text { Volume, costs } \\
\text { appropriateness } \\
\text { of ordering, QI }\end{array}$ & $x$ & $x$ & $x$ \\
\hline $\begin{array}{l}\text { Bernal-Del- } \\
\text { grado } \\
\text { et al., } \\
2002\end{array}$ & $\begin{array}{l}\text { Change prescrib- } \\
\text { ing behavior to } \\
\uparrow \text { medical } \\
\text { practice }\end{array}$ & $\begin{array}{l}\text { Physicians, other } \\
\text { Multiple }\end{array}$ & $\begin{array}{l}\text { Outreach visit with/ } \\
\text { without } \\
\text { EB-brochure }\end{array}$ & $K+R$ & $\begin{array}{l}\text { Change in } \\
\text { prescription } \\
\text { pattern, cost per } \\
\text { prescription }\end{array}$ & $x$ & $x$ & \\
\hline $\begin{array}{l}\text { Bhatia } \\
\text { et al., } \\
2013\end{array}$ & $\begin{array}{l}\downarrow \text { Proportion of } \\
\text { inappropriate } \\
\text { echocardiograms }\end{array}$ & $\begin{array}{l}\text { Medical students, } \\
\text { residents, physicians } \\
\text { General medicine }\end{array}$ & $\begin{array}{l}\text { AUC-lecture, } \\
\text { pocket card } \\
\text { providing tips, } \\
\text { utilization and } \\
\text { feedback on } \\
\text { appropriateness }\end{array}$ & $K+R$ & $\begin{array}{l}\text { Volume, } \\
\text { appropriateness } \\
\text { of ordering, } \\
\text { knowledge and } \\
\text { attitude }\end{array}$ & $x$ & & A \\
\hline $\begin{array}{l}\text { Blackstone } \\
\text { et al., } \\
1995\end{array}$ & $\begin{array}{l}\text { Educate in the } \\
\text { area of cost } \\
\text { awareness and } \\
\text { containment }\end{array}$ & $\begin{array}{l}\text { Residents } \\
\text { Surgery }\end{array}$ & $\begin{array}{l}\text { Guidelines and } \\
\text { recommendation, } \\
\text { multidisciplinary } \\
\text { discussions }\end{array}$ & $R+S$ & Costs & & $x$ & \\
\hline $\begin{array}{l}\text { Bornard } \\
\text { et al., } \\
2011\end{array}$ & $\begin{array}{l}\uparrow \text { Quality of } \\
\text { prescribing by } \downarrow \\
\text { unnecessary } \\
\text { prescribing }\end{array}$ & $\begin{array}{l}\text { Residents, physicians } \\
\text { Intensive care unit }\end{array}$ & $\begin{array}{l}\text { Feedback and } \\
\text { experts' advice to } \\
\text { improve, sessions on } \\
\text { medical topics, } \\
\text { multidisciplinary } \\
\text { discussions }\end{array}$ & $\mathrm{K}+\mathrm{R}+\mathrm{S}$ & $\begin{array}{l}\text { Volume, } \\
\text { appropriateness } \\
\text { of antibiotic } \\
\text { prescribing }\end{array}$ & $x$ & & $X$ \\
\hline
\end{tabular}


Appendix 2.3:

Descriptive overview of included articles (continued)

\begin{tabular}{|c|c|c|c|c|c|c|c|c|}
\hline \multirow{2}{*}{$\begin{array}{l}\text { Author } \\
\text { Year }\end{array}$} & \multirow[t]{2}{*}{ Aim } & \multirow{2}{*}{$\begin{array}{l}\text { Target group } \\
\text { Specialty }\end{array}$} & \multirow[t]{2}{*}{ Focus of intervention } & \multirow{2}{*}{$\begin{array}{l}\text { Frame- } \\
\text { work }\end{array}$} & \multirow{2}{*}{$\begin{array}{l}\text { Main outcome } \\
\text { measurement }\end{array}$} & \multicolumn{3}{|c|}{ Category } \\
\hline & & & & & & $\mathrm{V}$ & $\$$ & Q \\
\hline $\begin{array}{l}\text { Braido } \\
\text { et al., } \\
2012\end{array}$ & $\begin{array}{l}\uparrow \text { Guideline } \\
\text { knowledge and } \\
\text { adherence }\end{array}$ & $\begin{array}{l}\text { Physicians } \\
\text { General practice }\end{array}$ & $\begin{array}{l}\text { Economic analysis- } \\
\text { course regarding } \\
\text { prescribing and } \\
\text { resource utilization }\end{array}$ & $\mathrm{K}$ & $\begin{array}{l}\text { Costs, improved } \\
\text { knowledge of } \\
\text { guideline }\end{array}$ & $x$ & & $x$ \\
\hline $\begin{array}{l}\text { Cammisa } \\
\text { et al., } \\
2011\end{array}$ & $\begin{array}{l}\uparrow \text { Quality by } \\
\downarrow \text { overuse }\end{array}$ & $\begin{array}{l}\text { Physicians } \\
\text { Primary care }\end{array}$ & $\begin{array}{l}\text { Expert reflection on } \\
\text { utilization, advice, } \\
\text { guidelines }\end{array}$ & $K+R$ & Volume & $x$ & & \\
\hline $\begin{array}{l}\text { Chan- } \\
\text { dawarkar } \\
\text { et al., } \\
2007\end{array}$ & $\begin{array}{l}\uparrow \text { Cost } \\
\text { awareness }\end{array}$ & $\begin{array}{l}\text { Residents } \\
\text { Surgery }\end{array}$ & Costs pocket card & $\mathrm{K}$ & $\begin{array}{l}\text { Knowledge of } \\
\text { costs }\end{array}$ & & & \\
\hline $\begin{array}{l}\text { Colbert } \\
\text { et al., } \\
2010\end{array}$ & $\begin{array}{l}\uparrow \text { Awareness } \\
\text { and responsive- } \\
\text { ness to system } \\
\text { of health care }\end{array}$ & $\begin{array}{l}\text { Medical students } \\
\text { Gynecology }\end{array}$ & $\begin{array}{l}\text { Delivering care to } \\
\text { patients who do not } \\
\text { have health } \\
\text { insurance }\end{array}$ & S & $\begin{array}{l}\text { Students' experi- } \\
\text { ences and } \\
\text { awareness }\end{array}$ & & & \\
\hline $\begin{array}{l}\text { Collins } \\
\text { et al., } \\
1997\end{array}$ & $\begin{array}{l}\text { 个Accuracy and } \\
\text { appropriateness } \\
\text { of prescribing }\end{array}$ & $\begin{array}{l}\text { Physicians, } \\
\text { other } \\
\text { Unknown }\end{array}$ & $\begin{array}{l}\text { Patient-specific } \\
\text { information on } \\
\text { inappropriate } \\
\text { prescribed drugs } \\
\text { and guideline } \\
\text { information }\end{array}$ & K & $\begin{array}{l}\text { Costs of } \\
\text { prescribing }\end{array}$ & & $\mathrm{x}$ & \\
\hline $\begin{array}{l}\text { Das } \\
\text { et al., } \\
2010\end{array}$ & $\begin{array}{l}\uparrow V \text { itamin } \\
\text { prescribing } \\
\downarrow \text { wastage of } \\
\text { money }\end{array}$ & $\begin{array}{l}\text { Physicians } \\
\text { Multiple }\end{array}$ & $\begin{array}{l}\text { Prescriber focus } \\
\text { group, feedback data } \\
\text { and EBM-reflection }\end{array}$ & $K+R$ & $\begin{array}{l}\text { Volume, appropri- } \\
\text { ateness of } \\
\text { prescribing }\end{array}$ & $x$ & & $x$ \\
\hline $\begin{array}{l}\text { Davidoff } \\
\text { et al., } \\
1989\end{array}$ & $\begin{array}{l}\uparrow \\
\text { Decision-making }\end{array}$ & $\begin{array}{l}\text { Residents } \\
\text { unknown }\end{array}$ & $\begin{array}{l}\text { Course teaching } \\
\text { cost-containment } \\
\text { topics or probability } \\
\text { theory }\end{array}$ & $\mathrm{K}$ & $\begin{array}{l}\text { Volume of } \\
\text { ordering }\end{array}$ & $x$ & & \\
\hline $\begin{array}{l}\text { de Leon } \\
\text { et al., } \\
2013\end{array}$ & $\begin{array}{l}\downarrow \text { Rate of inap- } \\
\text { propriate use of } \\
\text { drugs }\end{array}$ & $\begin{array}{l}\text { Residents, physicians, } \\
\text { other } \\
\text { Intensive Care Unit }\end{array}$ & $\begin{array}{l}\text { Guidelines and } \\
\text { prescribing- } \\
\text { indications, } \\
\text { educational sessions, } \\
\text { EB facilitator-guide } \\
\text { for discussion }\end{array}$ & $\mathrm{K}+\mathrm{R}+\mathrm{S}$ & $\begin{array}{l}\text { Appropriateness } \\
\text { of prescribing }\end{array}$ & $x$ & & $x$ \\
\hline
\end{tabular}


Appendix 2.3:

Descriptive overview of included articles (continued)

\begin{tabular}{|c|c|c|c|c|c|c|c|c|}
\hline \multirow{2}{*}{$\begin{array}{l}\text { Author } \\
\text { Year }\end{array}$} & \multirow[t]{2}{*}{ Aim } & \multirow{2}{*}{$\begin{array}{l}\text { Target group } \\
\text { Specialty }\end{array}$} & \multirow[t]{2}{*}{ Focus of intervention } & \multirow{2}{*}{$\begin{array}{l}\text { Frame- } \\
\text { work }\end{array}$} & \multirow{2}{*}{$\begin{array}{l}\text { Main outcome } \\
\text { measurement }\end{array}$} & \multicolumn{3}{|c|}{ Category } \\
\hline & & & & & & V & $\$$ & $\mathrm{Q}$ \\
\hline $\begin{array}{l}\text { Dowling } \\
\text { et al., } \\
1989\end{array}$ & $\begin{array}{l}\downarrow \text { Not indicated } \\
\text { testing }\end{array}$ & $\begin{array}{l}\text { Residents } \\
\text { Family practice }\end{array}$ & $\begin{array}{l}\text { Feedback, EBM- } \\
\text { indications, cost } \\
\text { effectiveness theme } \\
\text { for preclinical talks }\end{array}$ & $\mathrm{K}+\mathrm{R}+\mathrm{S}$ & $\begin{array}{l}\text { Volume of test } \\
\text { ordering }\end{array}$ & $\mathrm{X}$ & & \\
\hline $\begin{array}{l}\text { Ellemdin } \\
\text { et al., } \\
2011\end{array}$ & $\begin{array}{l}\downarrow \text { Costs of } \\
\text { excessive } \\
\text { laboratory tests }\end{array}$ & $\begin{array}{l}\text { Physicians } \\
\text { Internal Medicine }\end{array}$ & $\begin{array}{l}\text { Ordering costs, } \\
\text { write costs on } \\
\text { ordering form }\end{array}$ & $\mathrm{K}$ & $\begin{array}{l}\text { Volume, costs of } \\
\text { care }\end{array}$ & $\mathrm{X}$ & $x$ & \\
\hline $\begin{array}{l}\text { Elligsen } \\
\text { et al., } \\
2010\end{array}$ & $\begin{array}{l}\downarrow \text { Inappropriate } \\
\text { use of } \\
\text { antimicrobials }\end{array}$ & $\begin{array}{l}\text { Physicians, other } \\
\text { Intensive care unit }\end{array}$ & $\begin{array}{l}\text { Expert reflection on } \\
\text { therapy, feedback to } \\
\text { physician }\end{array}$ & $\mathrm{R}$ & $\begin{array}{l}\text { Costs of hospital } \\
\text { admission }\end{array}$ & & $\mathrm{X}$ & \\
\hline $\begin{array}{l}\text { Englander } \\
\text { et al., } \\
2006\end{array}$ & $\begin{array}{l}\uparrow \text { Care that is of } \\
\text { optimal value }\end{array}$ & $\begin{array}{l}\text { Residents } \\
\text { Pediatrics }\end{array}$ & $\begin{array}{l}\text { Knowledge of costs } \\
\text { savings, meeting } \\
\text { regarding cost savings } \\
\text { and system-changes } \\
\text { for online ordering }\end{array}$ & $\mathrm{K}$ & $\begin{array}{l}\text { Volume, costs of } \\
\text { ordering }\end{array}$ & $\mathrm{X}$ & $\mathrm{X}$ & \\
\hline $\begin{array}{l}\text { Fakih } \\
\text { et al., } \\
2010\end{array}$ & $\begin{array}{l}\downarrow \text { Unnecessary } \\
\text { urinary catheter } \\
\text { placements }\end{array}$ & $\begin{array}{l}\text { Residents, physicians } \\
\text { Emergency department }\end{array}$ & $\begin{array}{l}\text { EB-clinical guideline } \\
\text { and indications, } \\
\text { pocket card, } \\
\text { reflection }\end{array}$ & $\mathrm{K}+\mathrm{R}$ & $\begin{array}{l}\text { Costs, } \\
\text { appropriateness of } \\
\text { lab-ordering }\end{array}$ & & $x$ & $X$ \\
\hline $\begin{array}{l}\text { Ferris } \\
\text { et al., } \\
2005\end{array}$ & $\begin{array}{l}\uparrow \text { Quality and } \\
\downarrow \text { costs of care }\end{array}$ & $\begin{array}{l}\text { Physicians } \\
\text { Primary care }\end{array}$ & $\begin{array}{l}\text { Case-based } \\
\text { recommendation for } \\
\text { prescribing / tests }\end{array}$ & $\mathrm{K}$ & $\begin{array}{l}\text { Volume, } \\
\text { appropriateness of } \\
\text { ordering }\end{array}$ & $x$ & & $x$ \\
\hline $\begin{array}{l}\text { Gist } \\
\text { et al., } \\
1997\end{array}$ & $\begin{array}{l}\downarrow \text { Unnecessary } \\
\text { breast biopsies } \\
\uparrow \text { early } \\
\text { detection of } \\
\text { breast cancer }\end{array}$ & $\begin{array}{l}\text { Physicians } \\
\text { Multiple }\end{array}$ & $\begin{array}{l}\text { EB-clinical algorithm } \\
\text { with follow-up } \\
\text { mailing }\end{array}$ & $\mathrm{K}$ & $\begin{array}{l}\text { Health care } \\
\text { utilization based } \\
\text { on medical claims }\end{array}$ & $\mathrm{X}$ & & \\
\hline $\begin{array}{l}\text { Gregory } \\
\text { et al., } \\
1999\end{array}$ & $\begin{array}{l}\downarrow \text { Rate of } \\
\text { cesarean } \\
\text { sections without } \\
\downarrow \text { in health care } \\
\text { quality }\end{array}$ & $\begin{array}{l}\text { Residents, physicians, } \\
\text { other } \\
\text { Gynecology }\end{array}$ & $\begin{array}{l}\text { Discussion } \\
\text { indications, utilization } \\
\text { feedback, focus } \\
\text { groups on attitudes } \\
\text { regardingVBAC }\end{array}$ & $K+R+S$ & $\begin{array}{l}\text { Positive predictive } \\
\text { value of biopsy }\end{array}$ & & & $x$ \\
\hline $\begin{array}{l}\text { Hart } \\
\text { et al., } \\
1997\end{array}$ & $\begin{array}{l}\text { 个 Unnecessary } \\
\text { prescribing }\end{array}$ & $\begin{array}{l}\text { Physicians } \\
\text { Multiple }\end{array}$ & Cost information & $\mathrm{K}$ & $\begin{array}{l}\text { Volume, costs, } \\
\text { appropriateness of } \\
\text { prescribing }\end{array}$ & $x$ & $x$ & $X$ \\
\hline
\end{tabular}


Appendix 2.3:

Descriptive overview of included articles (continued)

\begin{tabular}{|c|c|c|c|c|c|c|c|c|}
\hline \multirow{2}{*}{$\begin{array}{l}\text { Author } \\
\text { Year }\end{array}$} & \multirow[t]{2}{*}{ Aim } & \multirow{2}{*}{$\begin{array}{l}\text { Target group } \\
\text { Specialty }\end{array}$} & \multirow[t]{2}{*}{ Focus of intervention } & \multirow{2}{*}{$\begin{array}{l}\text { Frame- } \\
\text { work }\end{array}$} & \multirow{2}{*}{$\begin{array}{l}\text { Main outcome } \\
\text { measurement }\end{array}$} & \multicolumn{3}{|c|}{ Category } \\
\hline & & & & & & $\mathrm{V}$ & $\$$ & Q \\
\hline $\begin{array}{l}\text { Hux } \\
\text { et al., } \\
1999\end{array}$ & $\begin{array}{l}\text { 个 Rational drug } \\
\text { use by } \\
\text { promoting } \\
\text { EB-prescribing }\end{array}$ & $\begin{array}{l}\text { Physicians } \\
\text { Primary care }\end{array}$ & $\begin{array}{l}\text { Comparison- } \\
\text { feedback, guideline, } \\
\text { educational bulletin } \\
\text { with tips }\end{array}$ & $K+R$ & $\begin{array}{l}\text { Volume, costs of } \\
\text { prescribing }\end{array}$ & $x$ & $x$ & \\
\hline $\begin{array}{l}\text { ljo } \\
\text { et al., } \\
2011\end{array}$ & $\begin{array}{l}\uparrow \text { Quality of } \\
\text { care } \\
\downarrow \text { costs by } \\
\text { improving } \\
\text { prescribing }\end{array}$ & $\begin{array}{l}\text { Physicians, } \\
\text { other } \\
\text { Intensive care unit }\end{array}$ & $\begin{array}{l}\text { Feedback, peer- } \\
\text { discussions, Expert } \\
\text { reflection, } \\
\text { EB-newsletters }\end{array}$ & $K+R+S$ & $\begin{array}{l}\text { Cost of } \\
\text { prescribing, } \\
\text { QI }\end{array}$ & & $\mathrm{x}$ & $x$ \\
\hline $\begin{array}{l}\text { Krinsley } \\
\text { et al., } \\
2003\end{array}$ & $\begin{array}{l}\text { Safely } \\
\downarrow \text { utilization of } \\
\text { imaging }\end{array}$ & $\begin{array}{l}\text { Residents } \\
\text { Surgery }\end{array}$ & $\begin{array}{l}\text { Reflective questions } \\
\text { on ordering form }\end{array}$ & $R+S$ & $\begin{array}{l}\text { Volume of imaging, } \\
\text { QI }\end{array}$ & $x$ & & $x$ \\
\hline $\begin{array}{l}\text { Landgren } \\
\text { et al., } \\
\text { 1988 }\end{array}$ & $\begin{array}{l}\uparrow \\
\text { Appropriateness } \\
\text { of prescribing }\end{array}$ & $\begin{array}{l}\text { Physicians } \\
\text { Surgery }\end{array}$ & $\begin{array}{l}\text { Campaign, feedback } \\
\text { with discussion, } \\
\text { satirical video to } \\
\text { trigger discussion, } \\
\text { visit of academic } \\
\text { representative to } \\
\text { explain campaign and } \\
\text { answer questions }\end{array}$ & $R+S$ & $\begin{array}{l}\text { Volume, costs, } \\
\text { appropriateness } \\
\text { of prescribing }\end{array}$ & $x$ & $x$ & $x$ \\
\hline $\begin{array}{l}\text { Larmour } \\
\text { et al., } \\
2011\end{array}$ & $\begin{array}{l}\text { Maintain quality } \\
\text { while containing } \\
\text { healthcare costs }\end{array}$ & $\begin{array}{l}\text { Residents, physicians } \\
\text { Multiple }\end{array}$ & $\begin{array}{l}\text { Guidelines, academic } \\
\text { detailing, promotion } \\
\text { among staff }\end{array}$ & $\mathrm{K}+\mathrm{S}$ & $\begin{array}{l}\text { Costs, appropri- } \\
\text { ateness of } \\
\text { prescribing }\end{array}$ & $x$ & $x$ & \\
\hline $\begin{array}{l}\text { Légaré } \\
\text { et al., } \\
2012\end{array}$ & $\begin{array}{l}\downarrow \text { Overuse of } \\
\text { antibiotics }\end{array}$ & $\begin{array}{l}\text { Residents, physicians } \\
\text { Primary care }\end{array}$ & $\begin{array}{l}\text { Tutorial on patient- } \\
\text { communication, } \\
\text { workshop to } \\
\text { implement lessons in } \\
\text { practice }\end{array}$ & $\mathrm{K}$ & $\begin{array}{l}\text { Volume of pre- } \\
\text { scribing, decisional } \\
\text { conflict }\end{array}$ & $x$ & & $\mathrm{X}$ \\
\hline $\begin{array}{l}\text { Légaré } \\
\text { et al., } \\
2010\end{array}$ & $\begin{array}{l}\downarrow \text { Overuse of } \\
\text { antibiotics }\end{array}$ & $\begin{array}{l}\text { Physicians } \\
\text { Primary care }\end{array}$ & $\begin{array}{l}\text { EB-workshop, } \\
\text { feedback, reminders } \\
\text { of expected behavior }\end{array}$ & $K+R$ & $\begin{array}{l}\text { Decisional regret, } \\
\text { level of agreement }\end{array}$ & & & $x$ \\
\hline $\begin{array}{l}\text { Lyle } \\
\text { et al., } \\
1979\end{array}$ & $\begin{array}{l}\uparrow \text { Awareness of } \\
\text { costs } \\
\downarrow \\
\text { overutilization }\end{array}$ & $\begin{array}{l}\text { Medical student, } \\
\text { residents } \\
\text { Multiple }\end{array}$ & Utilization feedback & $K+R$ & $\begin{array}{l}\text { Volume, costs of } \\
\text { admission, QI }\end{array}$ & $x$ & $x$ & $x$ \\
\hline
\end{tabular}


Appendix 2.3:

Descriptive overview of included articles (continued)

\begin{tabular}{|c|c|c|c|c|c|c|c|c|}
\hline Author & Aim & Target group & Focus of intervention & Frame- & Main outcome & $\mathrm{Ca}$ & tegc & ory \\
\hline Year & & Specialty & & work & measurement & $\vee$ & $\$$ & $\mathrm{Q}$ \\
\hline $\begin{array}{l}\text { Mallows } \\
\text { et al., } \\
2013\end{array}$ & $\begin{array}{l}\downarrow \text { Ordering of } \\
\text { test }\end{array}$ & $\begin{array}{l}\text { Medical students, } \\
\text { residents, } \\
\text { physicians } \\
\text { Emergency department }\end{array}$ & $\begin{array}{l}\text { Utility-discussion, } \\
\text { information on costs } \\
\text { and quality of testing, } \\
\text { fine for ordering }\end{array}$ & $K+R+S$ & $\begin{array}{l}\text { Volume of testing, } \\
\text { post-hoc } \\
\text { discussions }\end{array}$ & & & \\
\hline $\begin{array}{l}\text { Manheim } \\
\text { et al., } \\
1990\end{array}$ & $\begin{array}{l}\uparrow \text { Cost } \\
\text { awareness } \\
\downarrow \text { costs }\end{array}$ & $\begin{array}{l}\text { Residents } \\
\text { Internal medicine }\end{array}$ & $\begin{array}{l}\text { Chart audits of high } \\
\text { costs, comparative } \\
\text { feedback on } \\
\text { generated costs, } \\
\text { evidence supported } \\
\text { discussions about } \\
\text { cost effectiveness of } \\
\text { care }\end{array}$ & $\mathrm{K}+\mathrm{R}+\mathrm{S}$ & $\begin{array}{l}\text { Volume, costs of } \\
\text { ancillary tests, QI }\end{array}$ & $x$ & $x$ & $x$ \\
\hline $\begin{array}{l}\text { Marconi } \\
\text { et al., } \\
2010\end{array}$ & $\begin{array}{l}\uparrow \text { Cost } \\
\text { awareness } \\
\uparrow \text { quality } \\
\text { cost } \\
\text { containment }\end{array}$ & $\begin{array}{l}\text { Residents } \\
\text { Pediatrics }\end{array}$ & EB-Guidelines & $\mathrm{K}$ & $\begin{array}{l}\text { Knowledge of } \\
\text { guideline }\end{array}$ & & & \\
\hline $\begin{array}{l}\text { McKay } \\
\text { et al., } \\
2011\end{array}$ & $\begin{array}{l}\downarrow \text { Unnecessary } \\
\text { prescribed drugs }\end{array}$ & $\begin{array}{l}\text { Residents, } \\
\text { physicians, } \\
\text { other } \\
\text { Unknown }\end{array}$ & $\begin{array}{l}\text { Training to provide } \\
\text { patient education, } \\
\text { course on } A B \text {-use, } \\
\text { strategies to } \\
\text { prescribe } \\
\text { appropriately }\end{array}$ & $\mathrm{K}+\mathrm{S}$ & $\begin{array}{l}\text { Volume of pre- } \\
\text { scribing, knowl- } \\
\text { edge of antibiotics }\end{array}$ & $x$ & & \\
\hline $\begin{array}{l}\text { McPhee } \\
\text { et al., } \\
1984\end{array}$ & $\begin{array}{l}\text { Control } \\
\uparrow \text { in costs } \\
\downarrow \text { unnecessary } \\
\text { ordering }\end{array}$ & $\begin{array}{l}\text { Medical students, } \\
\text { residents } \\
\text { Multiple }\end{array}$ & $\begin{array}{l}\text { Reviewing medical } \\
\text { records, cost- } \\
\text { feedback, price } \\
\text { information }\end{array}$ & $K+R$ & $\begin{array}{l}\text { Volume of hospital } \\
\text { services }\end{array}$ & & & \\
\hline $\begin{array}{l}\text { Miyakis } \\
\text { et al., } \\
2006\end{array}$ & $\begin{array}{l}\uparrow \text { Quality } \\
\downarrow \text { costs of } \\
\text { testing }\end{array}$ & $\begin{array}{l}\text { Residents, } \\
\text { physicians, } \\
\text { other } \\
\text { Unknown }\end{array}$ & $\begin{array}{l}\text { Feedback on } \\
\text { knowledge and } \\
\text { appropriateness, } \\
\text { discussion on } \\
\text { strategies to reduce } \\
\text { testing }\end{array}$ & $K+R+S$ & $\begin{array}{l}\text { Volume, } \\
\text { appropriateness } \\
\text { of laboratory } \\
\text { testing }\end{array}$ & $x$ & & $\mathrm{X}$ \\
\hline
\end{tabular}


Appendix 2.3:

Descriptive overview of included articles (continued)

\begin{tabular}{|c|c|c|c|c|c|c|c|c|}
\hline \multirow{2}{*}{$\begin{array}{l}\text { Author } \\
\text { Year }\end{array}$} & \multirow[t]{2}{*}{ Aim } & \multirow{2}{*}{$\begin{array}{l}\text { Target group } \\
\text { Specialty }\end{array}$} & \multirow[t]{2}{*}{ Focus of intervention } & \multirow{2}{*}{$\begin{array}{l}\text { Frame- } \\
\text { work }\end{array}$} & \multirow{2}{*}{$\begin{array}{l}\text { Main outcome } \\
\text { measurement }\end{array}$} & \multicolumn{3}{|c|}{ Category } \\
\hline & & & & & & V & $\$$ & $\mathrm{Q}$ \\
\hline $\begin{array}{l}\text { Moriates } \\
\text { et al., } \\
2013\end{array}$ & $\begin{array}{l}\uparrow \text { Cost } \\
\text { awareness } \\
\uparrow \text { attitude } \\
\text { toward cost- } \\
\text { control } \\
\text { cultivating cost- } \\
\text { effective } \\
\text { behavior }\end{array}$ & $\begin{array}{l}\text { Medical students, } \\
\text { residents, } \\
\text { physicians } \\
\text { Internal medicine }\end{array}$ & $\begin{array}{l}\text { Cost-awareness } \\
\text { session, price } \\
\text { information, review } \\
\text { of EB- guideline, } \\
\text { reflection on } \\
\text { ordering, stimulate } \\
\text { EB-care and cost- } \\
\text { effective care }\end{array}$ & $\mathrm{K}+\mathrm{R}+\mathrm{S}$ & $\begin{array}{l}\text { Attitude toward } \\
\text { relevance and } \\
\text { influence of } \\
\text { intervention on } \\
\text { care }\end{array}$ & & & \\
\hline $\begin{array}{l}\text { Niquille } \\
\text { et al., } \\
2010\end{array}$ & $\begin{array}{l}\text { Containing } \\
\text { prescribing costs }\end{array}$ & $\begin{array}{l}\text { Physicians, } \\
\text { other } \\
\text { General practice }\end{array}$ & $\begin{array}{l}\text { Discussion with } \\
\text { expert on (over)use, } \\
\text { risk/benefit }\end{array}$ & $\mathrm{K}+\mathrm{R}+\mathrm{S}$ & $\begin{array}{l}\text { Volume, costs, } \\
\text { appropriateness of } \\
\text { prescribing }\end{array}$ & $x$ & $x$ & $x$ \\
\hline $\begin{array}{l}\text { Okpara } \\
\text { et al., } \\
1995\end{array}$ & $\begin{array}{l}\text { 个Appropriate } \\
\uparrow \text { cost-effective } \\
\text { prescribing }\end{array}$ & $\begin{array}{l}\text { Residents, physicians, } \\
\text { other } \\
\text { Multiple }\end{array}$ & $\begin{array}{l}\text { EB-criteria, expert } \\
\text { recommendation for } \\
\text { improvement }\end{array}$ & $K+R$ & $\begin{array}{l}\text { Costs, rate of } \\
\text { accepted } \\
\text { recommendations, } \\
\text { QI }\end{array}$ & & $x$ & $x$ \\
\hline $\begin{array}{l}\text { Parrino } \\
\text { et al., } \\
1989\end{array}$ & $\begin{array}{l}\downarrow \text { Drug } \\
\text { utilization } \\
\downarrow \text { costs }\end{array}$ & $\begin{array}{l}\text { Physicians } \\
\text { Multiple }\end{array}$ & Cost-feedback & $\mathrm{R}$ & $\begin{array}{l}\text { Volume, costs of } \\
\text { prescribing }\end{array}$ & $x$ & $x$ & \\
\hline $\begin{array}{l}\text { Pasquale } \\
\text { et al., } \\
2004\end{array}$ & $\begin{array}{l}\uparrow \text { Care } \\
\downarrow \text { overall costs }\end{array}$ & $\begin{array}{l}\text { Physicians, Other } \\
\text { Infectious disease }\end{array}$ & $\begin{array}{l}\text { Expert reflection } \\
\text { regarding } \\
\text { inappropriate } \\
\text { ordering with } \\
\text { suggestion }\end{array}$ & $\mathrm{R}$ & $\begin{array}{l}\text { Volume, costs, } \\
\text { appropriateness } \\
\text { of prescribing }\end{array}$ & $x$ & $x$ & $x$ \\
\hline $\begin{array}{l}\text { Philips } \\
\text { et al., } \\
1986\end{array}$ & Cost saving & $\begin{array}{l}\text { Residents, physicians, } \\
\text { other } \\
\text { Multiple }\end{array}$ & $\begin{array}{l}\text { Newsletter with } \\
\text { price information } \\
\text { and suggestion for } \\
\text { improvement }\end{array}$ & $\mathrm{K}+\mathrm{S}$ & $\begin{array}{l}\text { Volume, costs } \\
\text { appropriateness, } \\
\text { of prescribing }\end{array}$ & $\mathrm{x}$ & $x$ & $x$ \\
\hline $\begin{array}{l}\text { Polinski } \\
\text { et al., } \\
201 \text { I }\end{array}$ & $\begin{array}{l}\downarrow \text { Cost of } \\
\text { prescribing }\end{array}$ & $\begin{array}{l}\text { Physicians } \\
\text { General practice }\end{array}$ & $\begin{array}{l}\text { Price information, } \\
\text { evidence to support } \\
\text { recommendation }\end{array}$ & $\mathrm{K}$ & $\begin{array}{l}\text { Volume of } \\
\text { accepted } \\
\text { suggestions }\end{array}$ & $x$ & & $x$ \\
\hline $\begin{array}{l}\text { Poppleton } \\
\text { et al., } \\
2003\end{array}$ & $\begin{array}{l}\text { Maintain } \\
\text { excellence and } \\
\text { efficiency } \\
\downarrow \text { costs }\end{array}$ & $\begin{array}{l}\text { Physicians } \\
\text { Multiple }\end{array}$ & $\begin{array}{l}\text { Clinical practice } \\
\text { guideline and } \\
\text { education regarding } \\
\text { the use }\end{array}$ & $\mathrm{K}$ & Costs, QI & & $x$ & $x$ \\
\hline
\end{tabular}


Appendix 2.3:

Descriptive overview of included articles (continued)

\begin{tabular}{|c|c|c|c|c|c|c|c|c|}
\hline \multirow{2}{*}{$\begin{array}{l}\text { Author } \\
\text { Year }\end{array}$} & \multirow[t]{2}{*}{ Aim } & \multirow{2}{*}{$\begin{array}{l}\text { Target group } \\
\text { Specialty }\end{array}$} & \multirow[t]{2}{*}{ Focus of intervention } & \multirow{2}{*}{$\begin{array}{l}\text { Frame- } \\
\text { work }\end{array}$} & \multirow{2}{*}{$\begin{array}{l}\text { Main outcome } \\
\text { measurement }\end{array}$} & \multicolumn{3}{|c|}{ Category } \\
\hline & & & & & & $\mathrm{V}$ & $\$$ & $\mathrm{Q}$ \\
\hline $\begin{array}{l}\text { Post } \\
\text { et al., } \\
2013\end{array}$ & $\begin{array}{l}\uparrow \text { Cost } \\
\text { awareness } \\
\uparrow \text { knowledge of } \\
\text { costs and value }\end{array}$ & $\begin{array}{l}\text { Residents } \\
\text { Internal medicine }\end{array}$ & $\begin{array}{l}\text { Feedback cost data, } \\
\text { reflection on } \\
\text { ordering }\end{array}$ & $K+R$ & $\begin{array}{l}\text { Knowledge of } \\
\text { costs, attitude } \\
\text { regarding costs }\end{array}$ & & & \\
\hline $\begin{array}{l}\text { Qureshi } \\
\text { et al., } \\
2011\end{array}$ & $\begin{array}{l}\text { 个 Quality of } \\
\text { drug } \\
\text { prescriptions }\end{array}$ & $\begin{array}{l}\text { Physicians } \\
\text { Primary care }\end{array}$ & $\begin{array}{l}\text { Guidelines, } \\
\text { prescribing-course } \\
\text { with health } \\
\text { economy-topics, } \\
\text { group discussions }\end{array}$ & $\mathrm{K}$ & $\begin{array}{l}\text { Volume, costs of } \\
\text { prescribing, com- } \\
\text { prehension of } \\
\text { educational topics }\end{array}$ & $\mathrm{X}$ & $\mathrm{x}$ & \\
\hline $\begin{array}{l}\text { Roth } \\
\text { et al., } \\
2001\end{array}$ & $\begin{array}{l}\text { Wise allocation } \\
\text { of resources }\end{array}$ & $\begin{array}{l}\text { Residents, physicians, } \\
\text { other } \\
\text { Multiple }\end{array}$ & $\begin{array}{l}\text { Cost information } \\
\text { flyer, expert } \\
\text { reflection, telephone } \\
\text { reminders }\end{array}$ & $\mathrm{K}+\mathrm{R}+\mathrm{S}$ & $\begin{array}{l}\text { Volume, costs of } \\
\text { prescribing }\end{array}$ & $X$ & $x$ & \\
\hline $\begin{array}{l}\text { Rotman } \\
\text { et al., } \\
1996\end{array}$ & $\begin{array}{l}\downarrow \text { Costs of } \\
\text { prescribing }\end{array}$ & $\begin{array}{l}\text { Residents } \\
\text { Internal medicine }\end{array}$ & $\begin{array}{l}\text { Cost information, } \\
\text { guideline, alerts with } \\
\text { suggestion }\end{array}$ & $K+R$ & $\begin{array}{l}\text { Volume, costs of } \\
\text { prescribing, } \\
\text { accepted recom- } \\
\text { mendations, user } \\
\text { satisfaction }\end{array}$ & $\mathrm{x}$ & $x$ & $\mathrm{X}$ \\
\hline $\begin{array}{l}\text { Rudy } \\
\text { et al., } \\
2001\end{array}$ & $\begin{array}{l}\downarrow \text { Cost } \\
\uparrow \text { quality of care }\end{array}$ & $\begin{array}{l}\text { Residents } \\
\text { Internal medicine }\end{array}$ & $\begin{array}{l}\text { Workshop on cost } \\
\text { effectiveness, } \\
\text { decision-making } \\
\text { with/without charges }\end{array}$ & $K+R$ & $\begin{array}{l}\text { Costs, appropri- } \\
\text { ateness of } \\
\text { decisions }\end{array}$ & & $x$ & $\mathrm{X}$ \\
\hline $\begin{array}{l}\text { Schroeber } \\
\text { et al., } \\
1984\end{array}$ & $\begin{array}{l}\downarrow \text { Unwarranted } \\
\text { orders } \\
\downarrow \text { charges }\end{array}$ & $\begin{array}{l}\text { Medical students, } \\
\text { residents } \\
\text { Multiple }\end{array}$ & $\begin{array}{l}\text { Cost information, } \\
\text { indications, utilization } \\
\text { and cost-feedback }\end{array}$ & $K+R$ & $\begin{array}{l}\text { Volume, costs, } \\
\text { appropriateness of } \\
\text { orders, QI }\end{array}$ & $f^{X}$ & $x$ & $\mathrm{X}$ \\
\hline $\begin{array}{l}\text { Self } \\
\text { et al., } \\
1984\end{array}$ & $\begin{array}{l}\uparrow \text { Rational use } \\
\downarrow \text { costs }\end{array}$ & $\begin{array}{l}\text { Medical students, } \\
\text { residents } \\
\text { Pulmonary medicine }\end{array}$ & $\begin{array}{l}\text { Guideline and } \\
\text { indications, cost } \\
\text { information }\end{array}$ & $\mathrm{K}+\mathrm{S}$ & $\begin{array}{l}\text { Volume, costs, } \\
\text { appropriateness of } \\
\text { prescription }\end{array}$ & X & $x$ & $\mathrm{X}$ \\
\hline $\begin{array}{l}\text { Shane } \\
\text { et al., } \\
1994\end{array}$ & $\begin{array}{l}\uparrow \text { Appropriate } \\
\uparrow \text { cost-effective } \\
\text { care }\end{array}$ & $\begin{array}{l}\text { Physicians } \\
\text { Multiple }\end{array}$ & $\begin{array}{l}\text { Expert reflection, } \\
\text { guidelines }\end{array}$ & $K+R$ & Volume, costs & $\mathrm{X}$ & $x$ & $\mathrm{X}$ \\
\hline $\begin{array}{l}\text { Sicotte } \\
\text { et al., } \\
1996\end{array}$ & $\begin{array}{l}\downarrow \text { inappropriate } \\
\text { resource use }\end{array}$ & $\begin{array}{l}\text { Physicians } \\
\text { Multiple }\end{array}$ & $\begin{array}{l}\text { Utilization feedback, } \\
\text { reflection on } \\
\text { feedback, } \\
\text { involvement of head } \\
\text { of department }\end{array}$ & $R+S$ & $\begin{array}{l}\text { Volume, costs, } \\
\text { usefulness of } \\
\text { feedback }\end{array}$ & $x$ & $x$ & \\
\hline
\end{tabular}




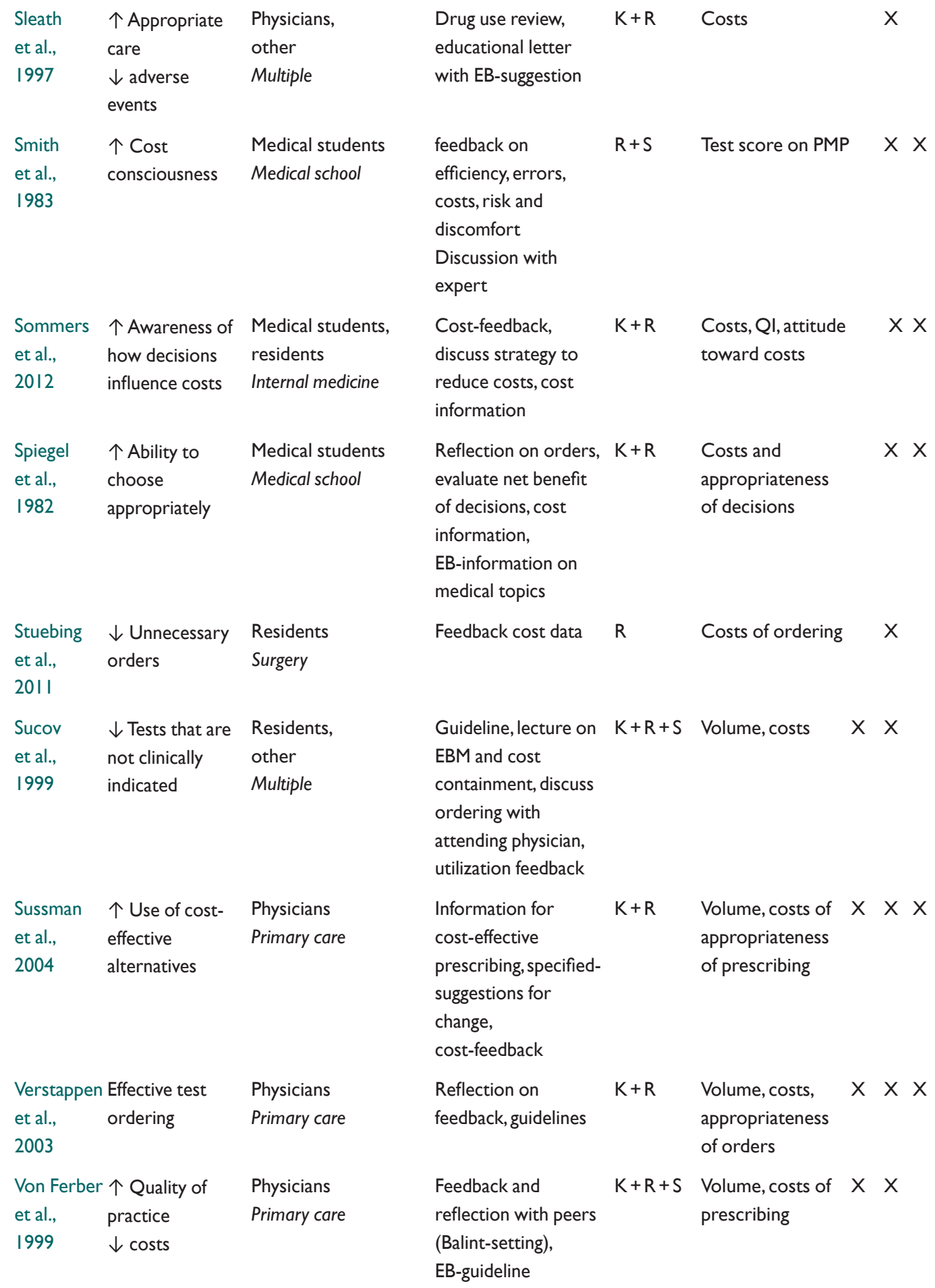




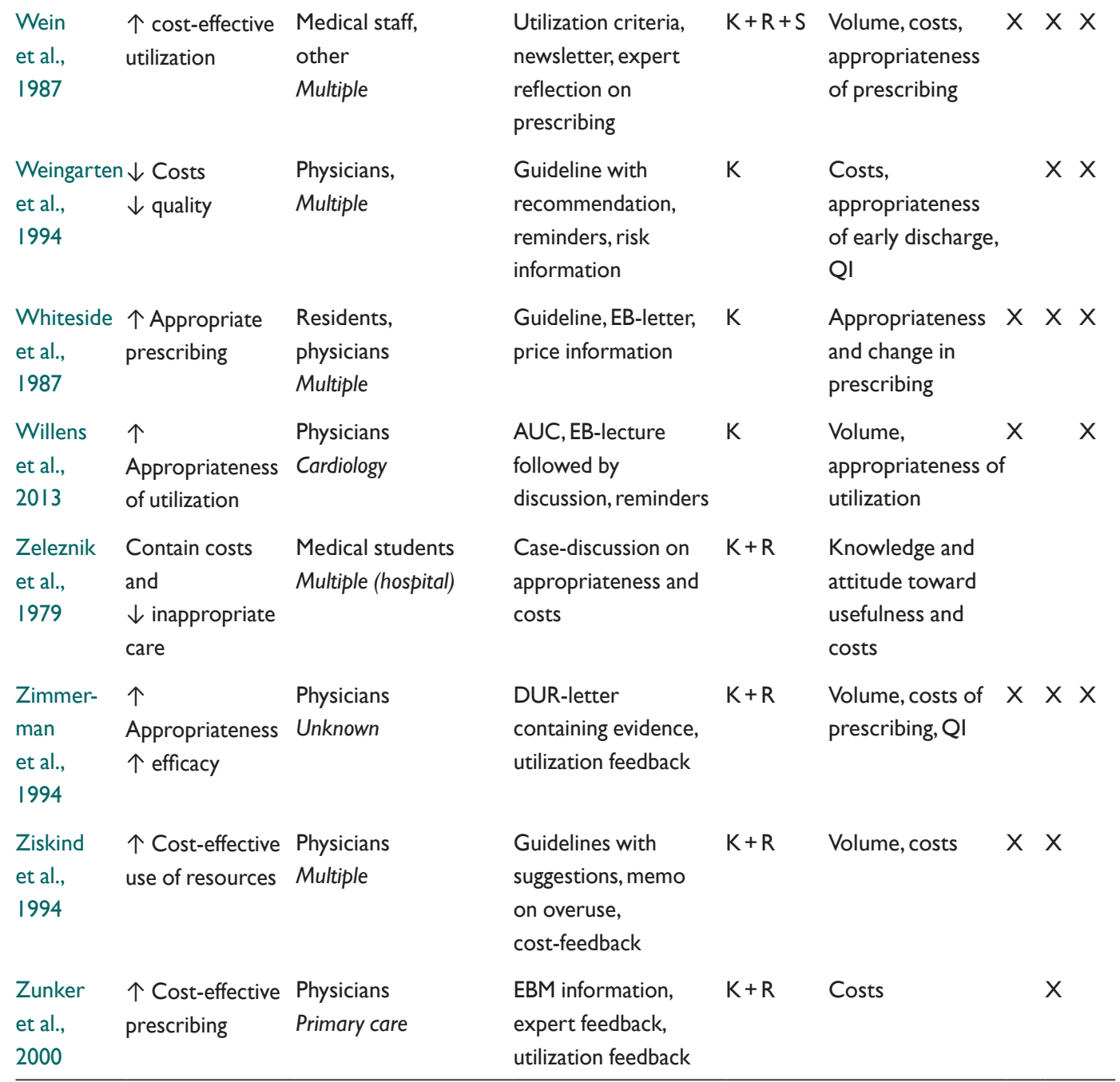

*Defined as adherence to guidelines, cost effectiveness, or approved by expert.

$\uparrow=$ Improve, stimulate, enhance, increase, promote

$\downarrow=$ Reduce, limit

$\mathrm{AB}=$ Antibiotic

$\mathrm{AUC}=$ Appropriate use criteria

Category of outcome measure: $\mathrm{V}=$ Volume, $\$=$ Monetary costs, Q= Quality

$\mathrm{DUR}=$ drug utilization review

$\mathrm{EB}=$ Evidence-based

$\mathrm{EBM}=$ Evidence-based medicine

Framework: $\mathrm{K}=$ knowledge transmission, $\mathrm{R}=$ reflective practice, $S=$ supportive environment
$\mathrm{ICU}=$ Intensive Care Unit

Multiple $=$ Multiple specialties were targeted by the intervention.

Other $=$ Other professionals than medical students, residents, and physician were target of intervention. For example, pharmacists, nursing staff, nurse practitioners, dieticians, healthcare managers.

PMP = Patient Management Problems

Primary care $=$ Primary Care and Family Medicine. QI = Quality Indicators. For example, length of stay, patient survival, mortality rate, morbidity rate, hospital readmission, days of therapy.

VBAC $=$ Vaginal Birth after C-Section 


\section{Appendix 2.4: Summary of the review process}

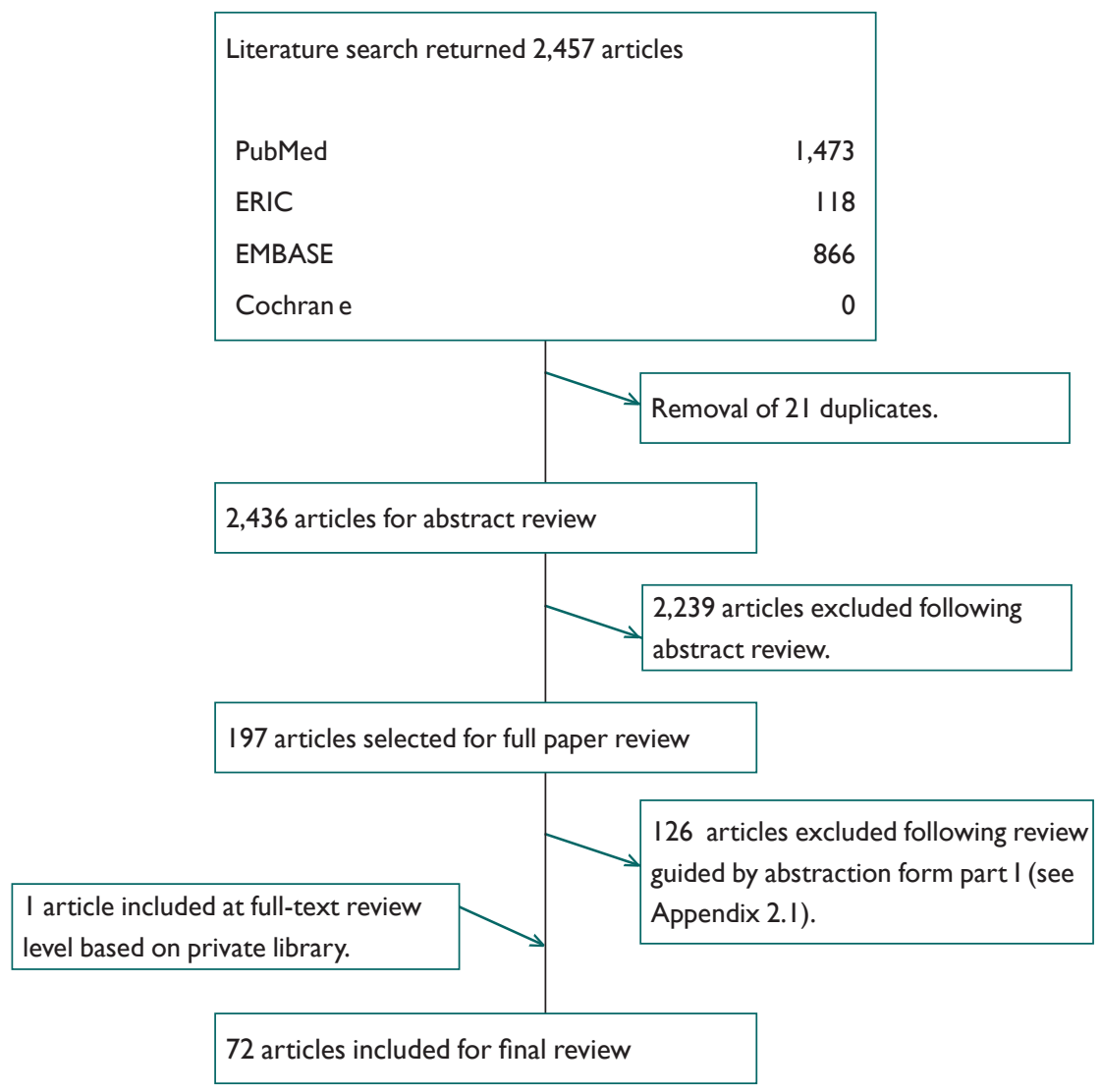





\section{Chapter 3 \\ The Struggle is Real How Residents Learn to Provide High-Value, Cost-Conscious Care}

Stammen, L., Slootweg, I., Stalmeijer, R., Janssen, L., Stassen, L., Scheele, F., \& Driessen, E. (20 I9). The Struggle Is Real:How Residents Learn to Provide High-Value, Cost-Conscious Care. Teaching and learning in medicine, $3 \mathrm{I}(4), 402-4 \mathrm{I}$. 


\section{Abstract}

Phenomenon: Rising health care expenditures threaten the accessibility and affordability of health care systems. Research has demonstrated that teaching (junior) physicians to deliver high-value, cost-conscious care can be effective when learning is situated in a supportive environment. This study aims to offer insight into how residents learn to provide high-value, cost-conscious care in the workplace and how the postgraduate training environment influences this learning.

Approach: Six homogeneous focus groups were held between August 2015 and July 2016 with 36 residents from six different residency programs (dermatology [ $n=5]$, elderly care [ $\mathrm{n}=8]$, family medicine $[n=5]$, internal medicine $[n=6]$, orthopedic surgery $[n=6]$, surgery $[n=6])$. An iterative, grounded theory approach was used to analyze the qualitative data.

Findings: Influential factors in learning of high-value, cost-conscious care delivery operated on three levels: individual resident, training program, and the workplace level. On the individual level we discerned three types of beliefs regarding HV3C. At the training program level, perceived determinants of learning included resident-supervisor interactions, involvement in decisionmaking over time, and exposure to variation in care delivery. At the workplace level, learning depended on the availability of professional health care expertise and on the presence of institutional policy.

Insights: Residents struggle to seize high-value, cost-conscious care learning opportunities in the workplace setting. Both residency training programs and workplaces can contribute to creating these learning opportunities. An important starting point is being aware of the different personal beliefs of residents and the approaches to high-value, cost-conscious care on the level of the training program and workplace. 


\section{Pursuing High-Value, Cost-Conscious Care}

\section{Introduction}

Mounting concerns over rising expenditures in health care, jeopardizing its sustainability, affordability, and accessibility, have galvanized insurance companies, governments, and hospital boards into curbing health care expenditures. In this endeavor, eliminating waste is expected to yield significant gains, as an estimated $20 \%$ of health care delivery can be considered wasteful. ${ }^{1-3}$ Physicians, who are involved in a large variety of health care decisions are thought to control $80 \%$ of health expenditures 4 making their role in achieving waste reduction a subject of keen interest. ${ }^{5-7}$ One potential strategy to reduce waste is to train physicians to deliver high-value, cost-conscious care (HV3C). In the last five years, the medical education realm has therefore emphasized the use of educational interventions designed to achieve this purpose. ${ }^{8-10}$ This focus has led to the launch of successful initiatives such as 'Choosing Wisely', the 'Top Five' list, ${ }^{11,12}$ and the American College of Physicians' 'High-Value Care initiative"13 aimed to train residents and physicians in order to simultaneously improve quality and eliminate health care waste. However, the effectiveness of these initiatives from an educational perspective has not been researched. A review of the 'working ingredients' of educational interventions that aim to train physicians in the delivery of high-value, cost-conscious care demonstrates that knowledge transmission, reflective practice, and a supportive environment are three important pillars of effective educational interventions. ${ }^{14}$

Despite the intentions of directors to offer HV3C training, incorporating it in postgraduate medical training programs progresses slowly. ${ }^{15}$, 16 This is due to the complexity of designing training aimed at effective knowledge transmission and reflective practice within an unsupportive environment. ${ }^{14,17,18} \mathrm{~A}$ supportive environment is essential as the hidden curriculum is known to hamper successful transfer of known skills into the workplace.19 Informal education matters, since residents spend most of their training in clinical settings and messages in the hidden curriculum can be far more powerful than the lessons taught by formal curricula. ${ }^{19,}{ }^{20}$ Without the support of informal education, formal educational interventions might miss the opportunity to effect sustained/internalized behavioral change. ${ }^{21}$ The purpose of this study is therefore to offer insight into I) how residents learn to provide $\mathrm{HV} 3 \mathrm{C}$ in the workplace and II) how their workplace environment influences this learning. Our findings can complement existing literature about workplace-based learning and enhance learning of $\mathrm{HV} 3 \mathrm{C}$ delivery during residency. 


\section{Methods}

\section{Study design}

Based on a literature review of educational interventions to promote HV3C delivery, ${ }^{14}$ we developed a semi-structured discussion guide designed to elicit the views of participants in focus groups (Appendix 3.1). We invited participants to share their personal experiences and to respond to their peers' views on $\mathrm{HV} 3 \mathrm{C}$ delivery. This technique provided us with a rich understanding of the topic under discussion. ${ }^{22-24}$ We first performed a dry run with eight residents in obstetrics and gynecology in order to test the order, content, and wording of the discussion guide, which needed no modifications. The Ethical Review Board of the Netherlands Association for Medical Education approved this study on June 18, 2015, under file number 547.

\section{Participants and setting}

We conducted our study in the Dutch postgraduate medical education setting, where at the time of our study, high-value, cost-conscious care was not part of the formal training curriculum. Table 5 and Table 6 present a general overview of the Dutch health care system and the Dutch medicine program.

Table 5: Overview of general characteristics of the Dutch health care system

Basic insurance is obligatory for every citizen

Payment through monthly fee and wage taxes (employer)

Additional insurance coverage is voluntary

Primary care is $100 \%$ covered

Out-of-pocket payment for basic care is capped at $€ 385^{*}$

Academic and non-academic hospitals

*unless citizens choose a higher threshold (max €850) for out-of-pocket payment in

Table 6: Overview of general characteristics of Dutch Medical Education continuum

\section{Structure of Dutch Medical Education continuum}

3 years preclinical training, Bachelor of Medicine

3 years clinical training, Master of Medicine

Intern year (optional. Can be more than I year)

3-6 years of residency training (after application and selection process)
Qualification after graduation

Physician, M.D.

Medical specialist 
Participants were 36 residents recruited from four different institutions and six different training programs (dermatology $[n=5]$, elderly care $[n=8]$, family medicine $[n=5]$, internal medicine [n=6], orthopedic surgery [n=6], surgery $[n=6]$ ) to participate in mono-professional focus groups. These residency training programs were selected to represent a broad range covering both surgical and nonsurgical specialties including hospital oriented and nonhospital-oriented training programs. We sent an email invitation and one reminder to 74 residents. Date and location of the focus group was based on residents' preferences and availability. Focus groups varied in size from five to eight members. Most participants had received more than two years of training at the time of data collection; Table 7 describes the characteristics of participants.

Table 7: Characteristics of focus group participants

\begin{tabular}{|c|c|c|c|c|c|}
\hline & Participants & Years & esidency $t$ & ing & $\begin{array}{l}\text { Years of clinical } \\
\text { working- } \\
\text { experience } \\
\text { pre-residency* }\end{array}$ \\
\hline $\begin{array}{l}\text { Type of specialty training (duration of } \\
\text { residency program) }\end{array}$ & $\mathrm{N}=$ & Mean & Spread & Modus & Mean \\
\hline Dermatology (6 years) & 5 & 3 & $1-4$ & 3 & 2 \\
\hline Elderly care medicine ( 3 years) & 8 & 3 & 3 & 3 & 2 \\
\hline Family care medicine ( 3 years) & 5 & 3 & 3 & 3 & I \\
\hline Internal Medicine (6 years) & 6 & 2 & $1-5$ & I & 1 \\
\hline Orthopedic surgery (6 years) & 6 & 4 & $2-6$ & 5 & 0 \\
\hline Surgery (6years) & 6 & 4 & $2-6$ & 4 & Unknown \\
\hline Total number of participants & 36 & & & & \\
\hline Characteristics of participants & $N(\%)$ & & & & \\
\hline Final year & $15(42 \%)$ & & & & \\
\hline$>50 \%$ of training completed & $23(64 \%)$ & & & & \\
\hline First year of training & $13(36 \%)$ & & & & \\
\hline Female & $23(64 \%)$ & & & & \\
\hline Male & $13(36 \%)$ & & & & \\
\hline
\end{tabular}

*In the Netherlands, there are opportunities to work in a health care institution (generally teaching or non-teaching hospital) after graduating medical school (M.D.) and before formally entering a residency program. This type of clinical workingexperience does not require formal teaching and is supervised by senior physicians. 


\section{Data collection \& analysis}

We collected data in the period between August 2015 and July 2016. During a 1.5-hour focus group session we discussed the residents' views, learning experiences, and personal struggles related to HV3C delivery. Focus groups were moderated by CC $(n=3)$ or AA $(n=3)$ and observed by AA $(n=3)$ or $\mathrm{HH}(\mathrm{n}=3)$. A moderator led the groups to keep focus on the research question while an observer watched the interactions to provide feedback for future focus groups. Data were audiorecorded and transcribed verbatim and analyzed anonymously. ATLAS.ti (version 8.2.0) was used to manage the data. In order to ensure methodological rigor, we took precautionary measures based on quality criteria such as credibility, transferability, dependability, and conformability. ${ }^{25-27}$ As characteristic for constructivist grounded theory approach, data collection and data analysis were done in an iterative manner, meaning that data collection was alternated with analysis in order to guide and deepen both collection and analysis. ${ }^{28}$ Two researchers (AA and BB) independently began analyzing the transcribed data using open coding in a line-by-line approach. ${ }^{29} \mathrm{In}$ this phase, $\mathrm{AA}$ and $\mathrm{BB}$ focused on descriptions, forbearing interpreting or analyzing the transcripts, and subsequently discussed their initial impressions and findings at several meetings. The next phase involved axial coding to identify causal conditions and residents' strategies. ${ }^{30}$ Additionally, AA and $\mathrm{BB}$ wrote analytical notes and reflective memos through all phases of data analysis. Constant comparison with alternating composition of the research team led to a collaborative analysis of data $^{29}$ and entailed comparing different transcripts, different codes, notes and memos, and different perceptions of the data. ${ }^{28}$ After six focus groups, we reached saturation (i.e., no new themes emerged from the data and a sufficient understanding of the themes was gained). ${ }^{24}$

Since the background of researchers has an influence on how data collection and analysis is executed ${ }^{31}$ we deliberately composed a research team with members of different disciplinary backgrounds (nursing, medicine, education) and relation to postgraduate training (medical student, resident, head of residency training, curriculum design). More specifically, the principal investigator, $\mathrm{AA}$, combines research with residency training. To contain any potential bias inferring with data analysis, the entire team reflected critically on AA's dual attachments, training experiences, and solidarity with fellow residents during the analytical process.

\section{Results}

Data analysis yielded factors that influence how residents learn to provide HV3C. These factors operated on three levels: the individual, the training program, and the workplace. 


\section{Pursuing High-Value, Cost-Conscious Care}

\section{Individual factors: residents' different beliefs regarding HV3C}

First, residents shared many commonalities in the way perceive HV3C. They all felt a strong sense of responsibility for providing the best care for their patients. Loyalty to patients was strong and money was considered 'a dirty word' if a patient's condition demanded a diagnostic test or treatment. Despite these similarities, we discovered three types of beliefs regarding HV3C held by residents.

\section{I'm just a frugal person}

To some residents, upholding HV3C goes to the heart of being an excellent physician. These residents were critical of themselves and of their environment in terms of the way health care was delivered. Residents embracing this attitude typically asked critical questions such as "will these tests change our treatment plan?" during group meetings and "would you be open to medication?" in patient encounters.

When asked why HV3C was so important to these residents, they explained it was a personal trait that manifested itself not only in their role as physician, but also in their private life. For example, as one resident stated, "I'm just frugal, for myself as well. We [the institution] are on a tight budget; I refuse to spend it all on medication, on a pill that costs five euro, when you can get one for 45 cents that has the same effect. I think that is wasteful. [...] You know, if nobody does it [be critical], it will never change." (R16)

\section{We have a professional responsibility, but...}

A second group of residents also valued the importance of HV3C, a topic that cropped up sometimes in interactions with patients, supervisors, and health care professionals. However, they did not always stick to the principle as they found it hard, sometimes even undesirable to translate into practice. Specifically, they struggled to explain HV3C considerations in their communication with patients, feeling that it would merely add to uncertainty. Additionally, they were often unsure whether to prioritize HV3C delivery above time management and learning goals, for example. They also tended to be accommodating when dealing with articulate, demanding patients who mistook more care for better care. As resident 21 said: "But it does run in the back of your mind, that I think [...] that I don't see any medical grounds [for more care] but yeah, am I going to spend half an hour chewing the fat about this?"

In all, these residents sometimes let time pressure, demanding patients, concerns over supervisors potentially overruling them, their wish to develop or maintain a patient-resident relationship, and fears of claims make them lose their focus on HV3C delivery. Although they initially aimed to provide $\mathrm{HV} 3 \mathrm{C}$, under external pressure their pro-HV3C aspirations waned. As resident 2 explained: "Some patients do not want to leave. They want another scan and I struggle with that. Sometimes you give in, sometimes you can talk them out of it." 


\section{Costs are not on my mind}

One of the residents who participated in the focus groups felt very strongly that it was incumbent upon residents to do everything in their power to help the patient, however costly. Moreover, this resident suggested that the only person able to judge the quality of care is the patient. Although this position startled the other focus group participants, it was clearly distinguishable as a personal held belief about HV3C, as reflected in the following quote: "Well, you have to, you have to help the patient and that patient is lying in the hospital because they want to be helped [...] I think you should pull out all the stops and not think, right...?” (R6)

\section{Factors associated with the training program}

The focus group discussions identified the factors associated with clinical educators and the training program that impacted the residents' learning curve. They explored both fruitful and missed learning opportunities and the group reflected on how the training program shaped their approach.

\section{Resident-supervisor interactions}

How residents learned HV3C delivery was contingent upon the availability and approaches of supervisors. Specifically, supervisors played a big role in reducing the anxiety that uncertainty and external pressures could prompt residents to start unnecessary procedures. Uncertainty was frequently the driver of over diagnosis or over treatment, so the residents found it useful to get feedback from supervisors and to reflect with them on the necessity of particular tests or the motives underlying the requests. However, there was little opportunity to do this, which sometimes led to unwanted situations. The impact of such adverse events could be profound. For example, one resident began ordering more tests in response to an unexpected death. "Um, I had an unexpected death of a young patient with chest pain, and I'd failed to request an ECG. After that, I ordered lots of ECGs, all in cases of chest pain, and I guess that's just the way it is at the moment." (R28)

The residents often mentioned that they sought advice from supervisors whom they knew would concur with their own views. As one resident put it: "You start positioning yourself a bit, manipulate a little, if you know what I mean. You know what you want to achieve, you trust your own ability at a given moment. And you know how to come across to so-and-so supervisor. [...] And you know [...] yeah okay, the supervisor would never let you do something that's not allowed, but if you want to do something, then you learn, yes - [how to] manipulate. Then you just go to the supervisor for their accord." (R3)

\section{Involvement in decision-making}

Residents said that being increasingly involved in decision-making promoted HV3C learning. Yet, opportunities to do so were not always forthcoming. On the one hand, internal obstacles 
could prevent them from participating in (multidisciplinary) discussions, as some residents felt it not yet their place, questioned their own abilities, or were uncertain as to when to introduce the topic of $\mathrm{HV} 3 \mathrm{C}$ into discussions that were already inherently complex.

On the other hand, however, supervisors did not always afford residents the opportunity to co-decide. Some supervisors were known to give residents little or no say in the development of a diagnostic or treatment plan, which residents perceived as a missed opportunity. Similarly, residents reported that certain staff members did not value their participation, causing them to feel frustrated about the delivered care. In cases where the supervisor dominated, or residents wanted to avoid discussion, residents adapted their diagnostic or treatment plan to their supervisors' preferences. At the same time, some supervisors encouraged residents to determine their own course of action. This is greatly appreciated, as resident seven said.

"Well, we sure do have supervisors who say, 'Okay, so this is what you propose? l'd do it this way. But let's do it your way and see what happens.'Those are the cases where I learn the most $[\ldots]$ in a safe environment of course." (R7)

A final reason why residents do not always reap the full benefit of co-decision opportunities is the short-term duration of their residency. Although they have plenty of opportunities to interact with patients during the residency, interactions were often momentary and long-term relationships with either supervisors or patients were scarce. This scarcity of follow-up opportunities caused residents to not always consciously consider patients' individual preferences and values, as the next quote illustrates.

"So, we literally pull out all the stops to offer someone the highest quality of care possible, but often without really, yeah, at least ostensibly without thinking about what would actually be best for that individual patient." (R32)

\section{Exposure to variation in care delivery}

Varying attitudes to HV3C delivery among residents, physicians, departments, and hospitals often gave an important impetus to the focus group discussions. Residents recognize that HV3C practices depend in part on the patient population, available resources, and organizational structure, as demonstrated by the quote below.

"You learn by spotting the differences. Like in the case of biopsies. They do them really differently in a non-academic center. It makes you wonder why we do it like that." (R36)

Variations in individual approaches of physicians also represented important learning cues for residents inspiring them to pose critical questions and start discussions with individual physicians 
or within professional teams. In doing so, especially the more senior residents learned to differentiate between the warranted and unwarranted approaches of physicians or organizations.

\section{Factors associated with the workplace}

\section{Availability of resources and expertise}

Residents were motivated to learn about HV3C and actively sought input in their workplace. The depth of their learning, however, depended heavily on the workplace learning opportunities, which took the form of mostly written information resources -such as guideline, protocols, and books- and professionals - serving as information sources and adequate role models of how to provide HV3C. Residents found it easier to tap into professional expertise in small or mediumsized non-academic centers. The smaller scale was conducive to familiarity, both inter-and intraprofessionally, and made it easier to cement the resident-expert bonds that create better opportunities for feedback and questions. Finally, residents noted that in certain workplaces staff seemed to be more critical of HV3C. For example, in this example below on prescribing, where residents were called out when staff felt care delivery did not match HV3C.

"It [ordering something expensive] does draw attention. If it's not the one prescribing the drug, then it will be the pharmacist who says, 'Gee, that drug you prescribed'....” (R/8)

(Response) "Yeah, somebody will call you." $(R / 3)$

\section{The presence of institutional policy}

Residents agreed that the presence of a clear institutional policy on HV3C helped them to determine the best course of action. An institutional policy would be defined as the presence of clear guidelines based on the standards of professional associations, health care institutions, or departments (e.g. protocols, treatment plans). These guidelines would provide residents with direction and focus when making HV3C-related decisions. Yet, an institutional policy regarding HV3C was often absent and dilemmas were usually resolved with the ad hoc aid of supervisors.

"Take transfusions. My current ward observes cut-off values very strictly. We often order them but refuse to administer above the cut-off point. We don't transfuse if a patient is tired.That's not enough [justification].The indications are less clear-cut on some wards but where l'm working now, it's no use trying. We don't think: Someone's tired, anemic, let's try two bags, maybe it'll help. Do you understand? There's a real difference."(R8) 


\section{Pursuing High-Value, Cost-Conscious Care}

Adding to the complexity of learning to provide HV3C were the mixed messages residents received at the workplace level regarding their role in HV3C. For instance, when management would stress in the first meeting that laboratory testing should be reduced, while they would stress in the second meeting that quality and following of guidelines should be prioritized over reduction of laboratory tests. Some residents also observed that hospital boards purportedly upholding HV3C values merely focused on money, not quality. Lack of clarity confused the residents, leading to some even resisting $\mathrm{HV} 3 \mathrm{C}$ delivery.

\section{Discussion}

Residencies are currently faced with the difficult task of producing physicians who are skilled in the delivering HV3C. ${ }^{15}, 32$ This study has sought to understand how residents learn to provide HV3C and how the postgraduate training environment influences their learning. Dutch residents, much like residents in other countries, are challenged to provide high-value care and take the costs into account. ${ }^{18,33}$ Answering the first research question, we found that residents' learning was driven by their personal beliefs regarding HV3C which led to equally different responses to $\mathrm{HV} 3 \mathrm{C}$-related dilemmas in clinical practice. When designing curricula, it is important to acknowledge different beliefs of residents and how these beliefs influence their $\mathrm{HV} 3 \mathrm{C}$ learning. With this knowledge in mind, residents might benefit from training tailored to their individual needs and personal learning curves, as advocated in the literature. ${ }^{34}$ Workplacebased learning could be enhanced if residents were encouraged to discuss their own beliefs and see how it affects the prioritization of their responsibilities as a physician.

Another powerful determinant of learning HV3C that emerged in our study was the presence of appropriate role models. Role modeling, combined with autonomy and reflection, has long been recognized as the most important pillar of workplace-based learning. ${ }^{71}{ }^{19}, 21$ In our study, effective role models were supervisors who valued residents' involvement in complex decision-making and gave them the opportunity to pursue their preferred plans. Furthermore, this study demonstrated that the influence of uncertainty and anxiety of residents hindered the delivery of HV3C. This influence of uncertainty is not limited to residents and has also been reported in previous research among physicians.35 Openly discussing these feeling of uncertainty and anxiety during supervision could therefore be important. To foster uptake of HV3C delivery in the workplace, it is therefore imperative that role models be informed about their influence and be involved in workplace-based learning. Role modeling HV3C is known to be scarce in residency training, due to lack of personal knowledge and training. ${ }^{18}$ Specific training may be required, teaching physicians to become effective role models of HV3C. It is not surprising that today's academic faculty experience difficulties in teaching HV3C-delivery, considering they themselves were not trained in these competencies. ${ }^{36}$ Finally, it may help to let residents observe and reflect on inter-supervisor variations. 
on care delivery during residency, ${ }^{17,37,38}$ and even throughout physicians' future career. ${ }^{38}$ Our second research question addressed the influence of the learning environment on the uptake of HV3C delivery. Previous research has demonstrated that physicians' HV3C learning must be situated in a supportive environment, ${ }^{7,20,33}$ and currently little emphasis is given to HV3C education in residency training. ${ }^{15,16,32,39-41}$ Once again our results emphasize the importance of a supportive workplace to facilitate learning. This criterion was met when the workplace operated with clear policies and residents could tap easily into the professionals' expertise. In this context, smaller health care organizations proved to be better breeding grounds for interdisciplinary networks with short communication lines facilitating access to expertise. This finding would make a case for residents rotating between health care organizations that vary in size, were it not for the fact that this would interfere with the need highlighted by our participants to establish long-term relationships with patients. A final recommendation arising from this research, which has been reiterated on various occasions elsewhere, ${ }^{42-44}$ is to create a safe learning environment: to enhance learning, residents must feel free to ask their supervisors critical questions and discuss HV3C-related dilemmas and residents' different beliefs.

\section{Strengths and limitations}

A major strength of this research is the variety of specialties involved in order to meet triangulation and transferability quality requirements. ${ }^{25-27}$ The participants not only came from six different residency programs, they also worked in various primary, secondary, and tertiary care institutions. This has led to rich data, enabling us to develop a broad theory, transcending the typical one hospital-based workplace. Furthermore, the wide range of expertise in the research team allowed us to discuss the data from several perspectives. Another strength is the rigor with which we performed our data analysis, owing to the use of constructivist grounded theory to address our research questions. ${ }^{30}$ Yet, a few limitations must be mentioned. First, participants may have been more aware of and favorable to $\mathrm{HV} 3 \mathrm{C}$, since focus group participation was voluntary. Nevertheless, the residents did not seem particularly enthusiastic at the start of the focus groups and sometimes showed uncertainty as to what could be defined as HV3C. Second, caution should be exercised when extrapolating the results to areas outside the Netherlands where this study was conducted, as the Dutch residency program, financial structure, and health care organization may differ from those existing in other regions of the world.

\section{Conclusion}

Residents struggle to seize high-value, cost-conscious care learning opportunities in the workplace setting. Both residency training programs and departmental policies can contribute to creating these learning opportunities. An important starting point is being aware of the different personal beliefs of residents and the approaches to high-value, cost-conscious care on the level of the training program and workplace. 


\section{Pursuing High-Value, Cost-Conscious Care}

\section{References}

1. Pate C DCM, Lutz S. The price of excess: identifying waste in health care spending. :

PriceWaterhouseCoopers; 2008 [cited 2018 21-12-2018]. Available from: http://www.pwc.com.

2. Berwick DM, Hackbarth AD. Eliminating waste in US health care. JAMA. 2012;307(14):1513-6.

3. Yong Pl SR, Olsen LA. The healthcare imperative: lowering costs and improving outcomes: workshop series summary. Washington D.C. : The National Academies Press (U.S.); 2010.

4. Crosson FJ. Change the microenvironment. Delivery system reform essential to control costs. Mod Healthc. 2009;39(17):20-1.

5. Shah N, Levy AE, Moriates C, Arora VM. Wisdom of the crowd: bright ideas and innovations from the teaching value and choosing wisely challenge. Acad Med. 2015;90(5):624-8.

6. Owens DK, Qaseem A, Chou R, Shekelle P, Clinical Guidelines Committee of the American College of P. High-value, cost-conscious health care: concepts for clinicians to evaluate the benefits, harms, and costs of medical interventions. Ann Intern Med. 2011;154(3):174-80.

7. Detsky AS, Verma AA. A new model for medical education: celebrating restraint. JAMA. 2012;308(13):1329-30.

8. Weinberger SE. Educating trainees about appropriate and cost-conscious diagnostic testing. Acad Med. 2011;86(11):1352.

9. Cooke M. Cost consciousness in patient care--what is medical education's responsibility? N Engl J Med. 2010;362(14):1253-5.

10. Weinberger SE. Providing high-value, cost-conscious care: a critical seventh general competency for physicians. Ann Intern Med. 2011;155(6):386-8.

11. Choosing Wisely Foundation T. Choosing Wisely Foundation [Webpage]. 2012 [updated 2019; cited 2018 08-03-2018]. Available from: http://www.choosingwisely.org/.

12. Brody H. Medicine's ethical responsibility for health care reform--the Top Five list. N Engl J Med. 2010;362(4):283-5.

13. Williams. High Value Care Curriculum (HVC) 4.0: ACP; 2012 [cited 2018 01-07-2018]. 4.0: [Available from: https://www.acponline.org/clinical-information/high-value-care/medical-educators-resources/ newly-revised-curriculum-for-educators-and-residents-version-40.]

14. Stammen LA, Stalmeijer RE, Paternotte E, Oudkerk Pool A, Driessen EW, Scheele F, et al. Training Physicians to Provide High-Value, Cost-Conscious Care: A Systematic Review. JAMA. 2015;314(22):2384-400.

15. Patel MS, Reed DA, Loertscher L, McDonald FS, Arora VM. Teaching residents to provide cost-conscious care: a national survey of residency program directors. JAMA Intern Med. 2014;174(3):470-2.

16. Ryskina KL, Holmboe ES, Shea JA, Kim E, Long JA. Physician Experiences With High Value Care in Internal Medicine Residency: Mixed-Methods Study of 2003-2013 Residency Graduates. Teach Learn Med. 2018;30(1):57-66.

17. Gupta R, Moriates C. Swimming Upstream: Creating a Culture of High-Value Care. Acad Med. 2017;92(5):598-601.

18. King BC, DiPace J, Naifeh M, Hammad H, Gerber LM, Abramson E. Pediatric Training Faculty and Resident Perceptions on Teaching High-Value, Cost-Conscious Care: A Multi-Institutional Study. Hosp Pediatr. 2017;7(9):547-52.

19. Glicken AD, Merenstein GB. Addressing the hidden curriculum: understanding educator professionalism. Med Teach. 2007;29(1):54-7.

20. Hafferty FW, Franks R. The hidden curriculum, ethics teaching, and the structure of medical education. Acad Med. 1994;69(11):861-71.

21. Coulehan J, Williams PC. Vanquishing virtue: the impact of medical education. Acad Med. 2001;76(6):598-605. 
22. Savin-Baden M. Qualitative Research - The Essential Guide to Theory and Practice. New York: Taylor \& Francis group; 2013.569 p.

23. Parker A. TJ. Focus group method and methodology: current practice and recent debate. International Journal of Research \& Method in Education. 292006. p. 23-37.

24. Stalmeijer RE, McNaughton N, Van Mook WN. Using focus groups in medical education research: AMEE Guide No. 91. Med Teach. 2014;36(11):923-39.

25. Tong A, Sainsbury P, Craig J. Consolidated criteria for reporting qualitative research (COREQ): a 32-item checklist for interviews and focus groups. Int J Qual Health Care. 2007;19(6):349-57.

26. Frambach JM, van der Vleuten CP, Durning SJ. AM last page. Quality criteria in qualitative and quantitative research. Acad Med. 2013;88(4):552.

27. O’Brien BC, Harris IB, Beckman TJ, Reed DA, Cook DA. Standards for reporting qualitative research: a synthesis of recommendations. Acad Med. 2014;89(9):1245-51.

28. Watling CJ, Lingard L. Grounded theory in medical education research: AMEE Guide No. 70. Med Teach. 2012;34(10):850-61.

29. Charmaz K. Constructing Grounded Theory: London Sage; 2006.

30. Corbin J. Basics in Qualitative Research: Thousand Oaks; Sage; 2008.

31. McMillan W. Researching Medical Education: John Wileys \& Sons; 2015.

32. Smith CD, Alliance for Academic Internal Medicine-American College of Physicians High V, CostConscious Care Curriculum Development C. Teaching high-value, cost-conscious care to residents: the Alliance for Academic Internal Medicine-American College of Physicians Curriculum. Ann Intern Med. 2012;157(4):284-6.

33. Sedrak MS, Patel MS, Ziemba JB, Murray D, Kim EJ, Dine CJ, et al. Residents' self-report on why they order perceived unnecessary inpatient laboratory tests. J Hosp Med. 2016;11(12):869-72.

34. Finlay L. Negotiating the Swamp: The Opportunity and Challange of Reflexivity in Research Practice. Qualitative Research. 2(2). London: Sage publications; 2002. p. 209-30.

35. Colla CH, Kinsella EA, Morden NE, Meyers DJ, Rosenthal MB, Sequist TD. Physician perceptions of Choosing Wisely and drivers of overuse. Am J Manag Care. 2016;22(5):337-43.

36. Wong BM, Holmboe ES. Transforming the Academic Faculty Perspective in Graduate Medical Education to Better Align Educational and Clinical Outcomes. Acad Med. 2016;91(4):473-9.

37. HoffTJ, Pohl H, Bartfield J. Creating a learning environment to produce competent residents: the roles of culture and context. Acad Med. 2004;79(6):532-9.

38. Asch DA, Nicholson S, Srinivas S, Herrin J, Epstein AJ. Evaluating obstetrical residency programs using patient outcomes. JAMA. 2009;302(12):1277-83.

39. Gould BE, O'Connell MT, Russell MT, Pipas CF, McCurdy FA. Teaching quality measurement and improvement, cost effectiveness, and patient satisfaction in undergraduate medical education: the UME-21 experience. Fam Med. 2004;36 Suppl:S57-62.

40. Dine CJ, Miller J, Fuld A, Bellini LM, Iwashyna TJ. Educating Physicians-in-Training About Resource Utilization and Their Own Outcomes of Care in the Inpatient Setting. J Grad Med Educ. 2010;2(2):175-80.

41. Ryskina KL, Halpern SD, Minyanou NS, Goold SD, Tilburt JC. The role of training environment care intensity in US physician cost consciousness. Mayo Clin Proc. 2015;90(3):313-20.

42. Lombarts KM, Heineman MJ, Scherpbier AJ, Arah OA. Effect of the learning climate of residency programs on faculty's teaching performance as evaluated by residents. PLoS One. 2014;9(1):e86512.

43. Genn JM. AMEE Medical Education Guide No. 23 (Part 1): Curriculum, environment, climate, quality and change in medical education-a unifying perspective. Med Teach. 2001;23(4):337-44.

44. Genn JM. AMEE Medical Education Guide No. 23 (Part 2): Curriculum, environment, climate, quality and change in medical education - a unifying perspective. Med Teach. 2001;23(5):445-54. 


\section{Appendix 3.I: Guide}

Focus group: Specialism, Date.

Before starting the focus group $\rightarrow$ participants sign informed consent form.

\section{Welcome:}

- Moderator and observer proposals

- Brief explanation of focus group

Research goal: brief explanation of the framework and objective of the study

"Thank you for taking part in this focus group. At Maastricht University, we are conducting research into HV3C and the role of education in this. I (IS/LS) will lead the group. We're about to start but before we do, I'd like to say that our aim is to gain further insight into your experiences and opinions regarding the theme of HV3C, I'd like to emphasize that there are no right or wrong answers. Different experiences and opinions are all useful for our study. That's also why we'd like you to respond to colleagues' experiences and opinions. At the end of the session, we will briefly discuss what you have said and commented on in response to the research questions." 


\begin{tabular}{|c|c|c|c|}
\hline The questions & Moderator's role & List of topics & $\begin{array}{l}\text { Theoretical } \\
\text { framework }\end{array}$ \\
\hline $\begin{array}{l}\text { Would you like to introduce } \\
\text { yourself? }\end{array}$ & $\mathrm{I} / \mathrm{L}$ lets everyone take a turn & $\begin{array}{l}\text { - Year of training Previous workplace, } \\
\text { academic / peripheral }\end{array}$ & \\
\hline What is HV3C? & $\mathrm{I} / \mathrm{L}$ invites all to share ideas & - What is the role of the patient in $\mathrm{HV} 3 \mathrm{C}$ ? & \\
\hline I) What do you think of HV3C? & $\mathrm{I} / \mathrm{L}$ summarize the ideas & $\begin{array}{l}\text { - What is the doctor's role? } \\
\text { - What is the role of the } \\
\text { organization? }\end{array}$ & \\
\hline
\end{tabular}

High-value, cost-conscious care is a broad concept. We would like to discuss with you HV3C; HV3C refers to care that aims to assess the benefits, harms, and costs of interventions and consequently to provide care that adds value. The focus today is on delivering HV3C to patients, the kind of care in which you, the doctors, play a vital role.

When do you come across HV3C I/L collects instances of HV3C

in daily practice?

2a) On a normal working day, when would you be giving $\mathrm{HV} 3 \mathrm{C}$ ?

2b) And how?

How do you learn to give HV3C?

3a) What do you consider when you are concerned with HV3C?

What do you need in your daily practice to ensure HV3C?

What do you need to decide what kind of care would be considered HV3C in daily practice? Is there anything left that we haven't dealt with sufficiently?

$\mathrm{I} / \mathrm{L}$ ensures that everyone gets a chance to have their own say 3b) Where or from whom did you learn to deliver

Awareness has their say

I/L broadens the discussion with in-depth questions

HV3C?

3c) What made you aware
Closing questions of things to do with HV3C?

- In or outside the hospital / workrelated or private

3d) What information or knowledge do you need for this?

What do you consider when you make a decision in light of HV3C?

- Patient preferences

- Knowledge of best practice / EBM

- Knowledge of costs / insurance Where did you obtain that knowledge?

3e) Do you ever look back on your actions from the standpoint of $\mathrm{HV} 3 \mathrm{C}$ ? What triggered you to reflect on your actions in the light of HV3C?

3f) What role does $\mathrm{HV} 3 \mathrm{C}$ play in the department and during supervision moments?

$3 g)$ Have you seen differences between different hospitals?

-Academic

- Peripheral

Where do you think those differences come from?
Knowledge transfer

Reflection on action taken in practice

Micro Stimulating environment

Macro Stimulating environment

What else do you think is important to share with the group? 


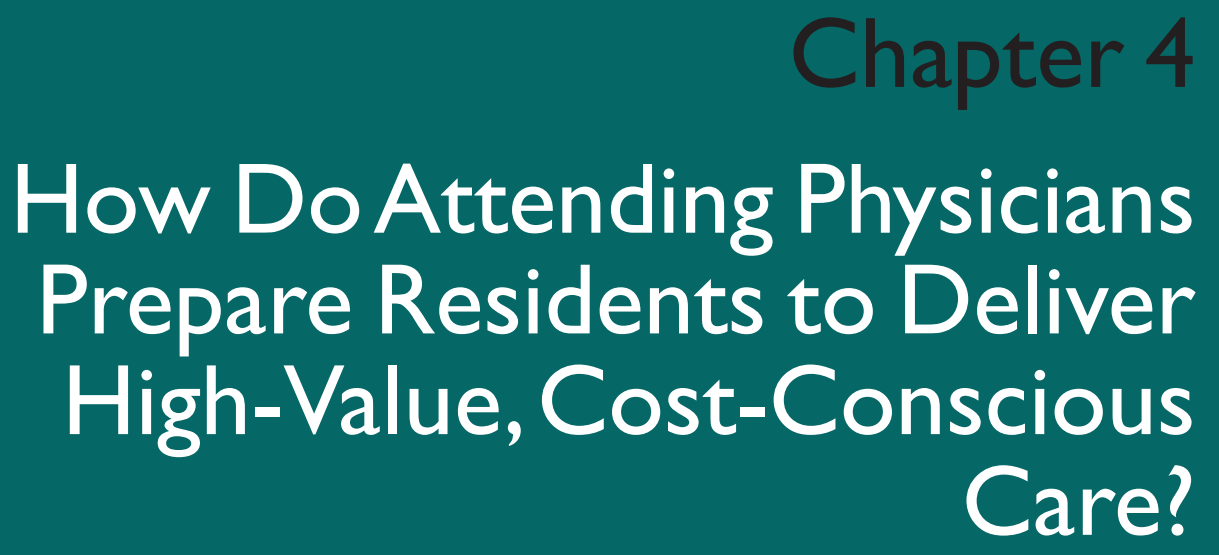

Stammen, L.A., Driessen, E.W., Notermans, C. C., Scheele, F., Stassen, L. P., \& Stalmeijer, R. E. (2019). How DoAttending Physicians Prepare Residents to Deliver High-Value, Cost-Conscious Care? Academic medicine:journal of the Association of American Medical Colleges May 2020 -Volume 95 - Issue 5. 


\section{Abstract}

Purpose: An estimated 20\% of health care expenditures are wasteful. Educational interventions aimed at reducing waste by delivering high-value, cost-conscious care (HV3C) often focus on the role of the physician. This study sought to understand how attending physicians, who have a central role in the workplace, prepare residents to provide HV3C.

Method: Semi-structured interviews were held between September 2016 and August 2017 with 12 attending physicians who supervise residents in the workplace. Participants were purposefully sampled from five institutions throughout the Netherlands to include surgical and nonsurgical attending physicians and hospital- and non-hospital-based physicians. Data collection and analysis were iterative, using principles of grounded theory, and were conducted by Maastricht University.

Results: The attending physician's approach to providing HV3C was an important factor in preparing residents in the workplace. Three differences became apparent: priority of HV3C training, feedback on HV3C, and obstacles to HV3C delivery. Results indicate that attending physicians use three teaching methods to teach HV3C delivery: Socratic questioning, role modeling, and setting limits. Training was often implicit and ad hoc.

Conclusions: How attending physicians deal with HV3C themselves influences how they prepare residents in the workplace. To optimize resident training, it may be important to create a supportive environment for HV3C delivery and training. Delivery could be supported by making $\mathrm{HV} 3 \mathrm{C}$ a shared goal for attending physicians and residents, thereby providing insight into clinical practice behavior and minimizing the influence of obstacles. Training could be optimized by supporting a variety of teaching methods suitable for daily teaching to stimulate continuous learning in residents. 


\section{Introduction}

When citizens and governments worldwide spend a substantial part of their budgets on health care, it is crucial that these expenditures are considered appropriate and sustainable over time. Unfortunately, estimates indicate that $20 \%$ of health care expenditures are wasteful. ${ }^{1,2}$ Interventions aimed at reducing wasteful health care services often focus on physicians. ${ }^{3,4}$ Physicians could use their medical expertise to safeguard the quality of care while providing care that is not only cost-conscious but also of high value., ${ }^{3,5}$ Indeed, physicians are included in highvalue, cost-conscious care (HV3C), defined as "care that aims to assess the benefits, harms, and costs of interventions and consequently to provide care that adds value." ${ }^{6}$ For the incorporation of HV3C in clinical practice, physicians should fulfill their role as stewards of resources and avoid misuse of health care services that do not benefit patient care. ${ }^{3}$ There is a rising awareness of the importance of $\mathrm{HV} 3 \mathrm{C}$ for current and future physicians, which is partly attributed to the launch of well-known initiatives such as Choosing Wisely, ${ }^{7}$ the Top Five list ${ }^{8}$ and the High Value Care Curriculum. ${ }^{9}$ To date, competency frameworks such as the Accreditation Council for Graduate Medical Education milestones ${ }^{10}$ and Canadian Medical Education Directives for Specialists ${ }^{11}$ have included HV3C, and it is generally accepted that medical education needs to rise to the challenge of training physicians to provide HV3C and incorporate it in curricula worldwide. ${ }^{5,12,13}$ Stammen et al. conducted a systematic review of educational interventions that addressed the topic of HV3C and found that effective educational interventions (1) provide health care workers with knowledge about HV3C, (2) stimulate reflection on care delivery from a HV3C perspective, and (3) create an environment that prioritizes HV3C delivery. ${ }^{14}$ Creating an environment that prioritizes $\mathrm{HV} 3 \mathrm{C}$ delivery relies partly on attending physicians, who play an important role in residency training as supervisors, role models, and members of the supportive environment in which residents work and learn. ${ }^{14-18}$ Unfortunately, previous studies indicate that residents often feel that $\mathrm{HV} 3 \mathrm{C}$ training is inadequate in the workplace. ${ }^{16}$ Most residency training is conducted in the workplace ${ }^{19}$ and lessons from graduate medical education can persist long into practice. ${ }^{13,20-}$ ${ }^{22}$ This research explored how attending physicians prepare residents to deliver HV3C, a topic that has not been studied previously. Increased understanding could help both residents and attending physicians to optimize the effect of HV3C training in the workplace.

\section{Method}

\section{Study design}

We invited attending physicians to participate in semi-structured interviews to share their experiences preparing future physicians for HV3C. This technique facilitates in-depth conversation, supported by examples, and provides space to discuss related topics in addition to 
those listed in the interview guide. ${ }^{23}$ The interview guide was based on a systematic review of the three essential elements of teaching HV3C (knowledge about HV3C, reflection on care delivery from a $\mathrm{HV} 3 \mathrm{C}$ perspective, and creation of a supportive environment that prioritizes HV3C delivery). ${ }^{14}$ (The interview guide is provided as Appendix 3.1). A pilot interview was held with an attending physician familiar with qualitative research, medical education, and HV3C. No modifications were made after the pilot session. Ethical approval was obtained from the ethical review board associated with the Netherlands Association for Medical Education on June 18, 2015, under file number 547. The research was conducted by Maastricht University.

\section{Setting}

The context of our study is postgraduate residency training in the Dutch health care system. Dutch health care is funded by a combination of compulsory social insurance and voluntary private insurance. Table 5 presents an overview of the financial structure of the Dutch health care system, and Table 6 presents an overview of the Dutch medical education continuum. In line with the increasing interest in HV3C worldwide, awareness of HV3C in the Dutch health care system is visible in government grants ${ }^{24}$ and in conferences, ${ }^{25}$ and it has officially been on the national training agenda since 2013. ${ }^{26,27}$ Despite this increased awareness, none of the medical centers participating in this study had a formal program to train residents in HV3C.

Table 5: Overview of general characteristics of the Dutch health care system

\section{Structure of Dutch health care system}

Basic insurance is obligatory for every citizen

Payment through monthly fee and wage taxes (employer)

Additional insurance coverage is voluntary

Primary care is $100 \%$ covered

Out-of-pocket payment for basic care is capped at $€ 385^{*}$

Academic and non-academic hospitals

*unless citizens choose a higher threshold (max €850) for out-of-pocket payment in

return for lower monthly fees.

Table 6: Overview of general characteristics of Dutch Medical Education continuum Structure of Dutch Medical Education continuum

Qualification after graduation

3 years preclinical training, Bachelor of Medicine

3 years clinical training, Master of Medicine

Physician, M.D.

Intern year (optional. Can be more than I year)

3-6 years of residency training (after application and selection process)

Medical specialist 


\section{Pursuing High-Value, Cost-Conscious Care}

\section{Sampling and data collection}

Between September 2016 and August 2017, we iteratively collected data by interviewing attending physicians. We purposively sampled ${ }^{28}$ attending physicians from various training programs, both surgical and nonsurgical and hospital and non-hospital-based. Six program directors assisted in identifying potential study participants from their training program. We asked the program directors to select two attending physicians from their department who were representative of the department and differed in their level of involvement (involvement or no involvement) in formal educational roles. We emailed invitations to all potential participants. In the email we included an informative letter that explained why we were approaching them and what their participation would involve as well as a form to document their informed consent. Two potential participants (both from one discipline) did not respond to the emailed invitation and subsequent reminders, so we asked the director of the training program in question to identify four other participants. Again, two did not respond. We completed 12 interviews with nine male $(75 \%)$ and three female (25\%) attending physicians based in teaching hospitals and working in six specialties: dermatology, elderly care, general practice, internal medicine, orthopedic surgery, and surgery. Participants worked in different regions of the Netherlands and were based in five different medical centers. Attending physicians who specialized in elderly care and family medicine were classified as nonhospital because they worked in either elderly care institutions or family medicine practices (comparable to outpatient care or ambulatory facilities). Seven participants (58\%) held educational roles such as clerkship coordinator or residency program director in their specialty. All participants were involved in supervising residents in daily practice, working in shifts, as is common in teaching hospitals. The interviews took place at a location convenient to the participant and were scheduled to last 60 minutes. The duration varied from 38 to 58 minutes. The first author, who is a family medicine resident, conducted all the interviews, which were audiotaped and then transcribed verbatim in Dutch. Quotes that were selected for the final manuscript were translated by an English language editor. ATLAS.ti (version 8.3.1., Scientific Software Development GmbH, Berlin, Germany) was used to manage the data.

\section{Data analysis}

Data were analyzed iteratively, based on the principles of constructivist grounded theory approach, beginning with line-by-line open coding..$^{29}$ The second step was axial coding, in which we identified the central codes and the relationships between codes. ${ }^{30}$ Finally, we used selective coding to develop a provisional coding scheme to better categorize and explore the conditions in which HV3C was addressed. We used the coding scheme, after further refinement by LAS, CCVIN, and RES, to review the transcripts. The whole team discussed the coding scheme and 
relationships between codes, which resulted in several key themes. After 12 interviews in total, data sufficiency was met, meaning that no new themes emerged from the data and we achieved an adequate understanding of the themes. ${ }^{28,31}$ Rigor of the study was ensured by adhering to the following principles throughout the study and writing process: credibility (through investigator triangulation, pilot interview), transferability (through describing context and sampling, relation to literature), dependability (through iterative data collection and analysis and data collection until saturation), and conformability (through reflexivity, analytical notes, and reflective memos). ${ }^{32}$ We also used the COREQ (consolidated criteria for reporting qualitative research) checklist as a reporting tool. ${ }^{33}$

In qualitative data analysis, it is important to consider the background of researchers, including their involvement in previous research and its influence on data collection and analysis. ${ }^{34}$ Our research team contained experts from several disciplines (medical education, gynecology, surgery, and family medicine) and various levels of medical training (medical student, resident, senior physician, and head of residency training). To maximize the contribution of these different backgrounds, the entire team was closely involved in data analysis. ${ }^{29}$

\section{Results}

Data analysis made apparent that (1) the attending physician's approach to HV3C influences (2) how he or she prepares residents to deliver HV3C in the workplace. This section discusses the findings on both aspects in turn.

\section{Attending physician's approach to HV3C}

For attending physicians, three notable differences became apparent in their approach to their own HV3C practice and to supervision: priority of HV3C training, feedback on HV3C delivery, and obstacles to HV3C delivery.

Priority of HV3C training. First, we noted a difference in the priority given to HV3C training in residency. This difference in priority was noted in the words chosen by participants ${ }^{35}$ that express a low priority (e.g., "the only occasion," "sometimes") compared with words that express a high priority (e.g., "drilled into residents," "always"). Some attending physicians addressed HV3C only when care delivery was perceived as "excessive" by the attending physicians; others aimed to continuously train residents in HV3C delivery: "Um, well actually it's the core of our profession, you know? I think it comes up in almost every case, in one way or another" (S4). Despite differences in the priority given by participants, all attending physicians aimed to 


\section{Pursuing High-Value, Cost-Conscious Care}

incorporate $\mathrm{HV} 3 \mathrm{C}$ both in their own care delivery and in training residents. They described how considering the risks, costs, and harms is essential to high-quality care and thus of the utmost importance for health care delivery. Although the financial cost of health care was also considered important, some participants explicitly rated it as secondary to quality of care or considered it a "dirty word."

The participants who continuously trained residents in HV3C delivery used a variety of teaching methods and made the training part of daily supervision. Teaching methods such as Socratic questioning, role modeling, and setting limits were used to prepare residents for the delivery of $\mathrm{HV} 3 \mathrm{C}$ and will be discussed more extensively later in the results section. During the interviews, these physicians supplied many instances of when they felt they were training residents in HV3C delivery, for example, when discussing a resident's care services, such as prescribing expensive medication or delivering care that did not meet the patient's preferences. We also identified physicians who declared that HV3C was important yet applied teaching methods to facilitate HV3C learning only occasionally: "Yes, I do it, but am I really aware of doing it? I wouldn't know" (S11). A few participants had difficulty providing examples of when they trained residents to deliver HV3C.

Feedback on HV3C delivery. In general, the participants were interested in receiving feedback on their HV3C behavior from colleagues, residents, interns, and other health care professionals. Additionally, they mentioned their need for supportive data on their own behavior and the opportunity to compare their data with the data of colleagues. Some participants had access to such data (e.g., frequency of complications, frequency of referrals, prescribing data) or had insight into costs (financial overview of own productivity) that were gleaned from feedback tools. Feedback tools gave them the ability to monitor their own behavior with or without the opportunity to compare and discuss these details with local and regional colleagues. At meetings that included the topic of local and regional testing, "... the residents are present, of course...." (S8). Participants who did have access to feedback tools perceived these tools as useful to determine and reflect on HV3C. Participants with no access to feedback tools knew about the use of feedback tools in other specialties, mostly in family medicine and elderly care. In the absence of such tools, participants perceived a lack of insight into their own care delivery, which was considered a real hindrance to critical reflection on HV3C delivery and their ability to train residents in such behavior.

Obstacles to HV3C delivery. Attending physicians mentioned two specific obstacles to HV3C delivery in daily practice: reluctance to discuss HV3C with patients and lack of knowledge about the cost of health care services. The reluctance to discuss HV3C, specifically the costs, occurred mostly during patient consultations. Participants often mentioned the patientphysician relationship as a reason for not discussing health care costs with patients; a few 
participants felt that it implied prioritizing money over quality. The physicians who did discuss costs openly explained that they wanted to do so because of "societal concerns about rising health care costs" (S9) and "how (expensive) medicine is not always desirable or beneficial for patients." (S1) In light of the patient-physician relationship, these participants could not give examples of conflicts arising when discussing costs with patients, although all emphasized that this delicate topic needed considerate communication. Residents working with these attending physicians were exposed to these conversations and encouraged to discuss costs themselves if deemed necessary.

Participants felt that gaps in knowledge included both inherent knowledge of health care costs and economics in general, as well as an inability to discover the cost of frequently used diagnostics or drugs. As one participant said, "The only difficult thing is that the costs are not very clear to me." (S6) The difficulty became apparent when patients, residents, students, or colleagues asked questions about the price of health care services, departmental health care costs, and coverage. Answering these questions remained difficult and attempts to unravel financial pathways in their workplace were often unsuccessful. In an attempt to deal with this obstacle, some physicians registered for external courses to help them gain insight in general health care economics, the factors contributing to the composition of price for health care services, and the effects of health care system coverage on health care costs.

\section{How attending physician prepares residents to deliver HV3C in the workplace}

Physicians spoke about aiming to integrate $\mathrm{HV} 3 \mathrm{C}$ in residency training in the workplace. Their examples of preparing residents for HV3C delivery can be classified in three main teaching methods: Socratic questioning, role modeling, and setting limits.

Socratic questioning. To ensure that residents pay attention to $\mathrm{HV} 3 \mathrm{C}$, attending physicians asked questions about $\mathrm{HV} 3 \mathrm{C}$ while supervising. The questions often focused on the cost of health care: "Do you have any idea of the price of this extra test?" (S2) Or they drew attention to the necessity of a particular service: "Will [the result of] this extra test change our treatment plan?" (S9) Attending physicians aimed to raise residents' awareness using open-ended questions, without trying to give information on what care would be best or by discussing their personal opinion of HV3C.

A frequently stated training strategy attending physicians used was to deduce with the resident why the resident made a particular treatment decision. These deductions were usually triggered by a case that did not meet the physician's standards; sometimes the residents proposed a case themselves. In some instances, attending physicians recognized a pattern in residents, such as 
"defensive medicine," "conflict-avoidant behavior," or "excessive testing." An example of conflictavoidant behavior given by an attending physician described a resident who always conceded to a patient's demands, even when the resident had concluded that the requested care was unnecessary or inappropriate. Together, physician and resident discussed the more global topic of typical behavioral patterns and professional identity: "Is that your style? Why is that? Why do you do that? How do you try to [say no]?” (S9) Other attending physicians discussed professional identity by asking questions such as, "Why did you give in to the patient's wishes? As a physician, is it hard for you to say no?" (S8) These training moments focused on the resident's attitude toward HV3C, expressed as "What kind of doctor do you want to be?" Data analysis revealed that not all attending physicians asked reflective questions regarding behavioral patterns and professional identity.

Role modeling. Attending physicians were aware of their impact on the residents' delivery of care and aimed to be role models for HV3C delivery in their daily practice. The participants tried to model communication skills, including persuasion and negotiation, which were seen as essential to HV3C delivery. Sometimes residents asked for support if they felt they needed help to convince patients of the inappropriateness of a request but failed to do so alone. On such occasions, the attending physician aimed to demonstrate $\mathrm{HV} 3 \mathrm{C}$ by explaining to the patient, in the presence of the resident, why specific diagnostic procedures or checks were not suitable and what the better alternatives were. In so doing, the attending physician aimed to transfer his or her communication skills to the residents. None of the participants explicitly discussed their role modeling with the residents; they assumed that the residents would recognize the teaching moment and its relation to HV3C.

"In terms of communication, I really hope that when they see how I explain things and eh, in my manner and body language, that they will get something out of it. I don't check that [whether they get something out of it].” (S12)

Role modeling also included leading by example within the department, such as placing restrictions on additional testing, exploring alternatives, and being critical of colleagues' behavior during handovers, multidisciplinary team meetings, or case discussions.

Setting limits. Attending physicians characterized junior residents as potential deliverers of wasteful care and in need of guidance by more senior physicians. Some participants provided guidance by closely monitoring care delivery (including additional testing) and limiting resident autonomy, for example, in prescribing specific drugs. As one said: "They're not in a position to prescribe those expensive drugs on their own. So that's where they're often called to a halt." (S12) Some workplaces preselected a list of preferred drugs (a formulary) to set limits on residents' prescribing patterns; residents were not allowed to prescribe drugs that were not on the list. 
Attending physicians used the patient perspective (i.e., intervention is harmful) more often than the economic perspective (i.e., intervention is costly) to justify the limits of a particular HV3C treatment to the resident and to advocate the importance of HV3C. For example, extensive laboratory testing was more easily accepted if attending physicians felt it would do the patient no harm. As residents became more senior, these limits would be removed because the residents were deemed capable of dealing with the total spectrum of services.

\section{Discussion}

Educational interventions aimed at reducing health care waste by delivering HV3C often focus on the role of the physician. This study sought to understand how attending physicians, who have a central role in the workplace, prepare residents to provide HV3C. Analyzing our data made it apparent that how an attending physician prepared residents was determined by the physician's own approach to HV3C: the priority he or she gave to HV3C training, which teaching methods he or she used, and which obstacles to HV3C delivery were perceived.

Results indicated that the attending physicians' approach to HV3C shaped how they prepare residents to deliver HV3C. Hence, the priority the participants gave to $\mathrm{HV} 3 \mathrm{C}$ training varied. All participants underlined the importance of HV3C to sustainable health care and recognized their personal role in preparing future physicians who can balance the costs, risks, and benefits of medical services for individual patients and society at large. This finding is important in the light of how one's beliefs, personal values, and experiences are known to strongly influence how one teaches. ${ }^{17,36-38}$ Additionally, it is known that when the training environment, with attending physicians as key figures, pays specific attention to HV3C and a shared goal in HV3C training, educational interventions are more effective. ${ }^{14,39}$ Therefore, in educational interventions, it might be wise to consider paying attention to the attending physicians' beliefs about HV3C and how the training environment is involved in $\mathrm{HV} 3 \mathrm{C}$ delivery.

Participants' personal beliefs about HV3C influenced how they prepared residents to deliver HV3C in the workplace. In their examples, Socratic questioning, role modeling, and setting limits were apparent as teaching methods for HV3C. These teaching methods are suitable for the situations' residents encounter in daily health care practice and can be labeled natural teaching moments. Nevertheless, the variety of teaching methods used to prepare residents to deliver $\mathrm{HV} 3 \mathrm{C}$ is limited compared with known teaching methods employed for other topics. For example, scaffolding (supporting residents in the delivery of $\mathrm{HV} 3 \mathrm{C}$, tailored to the residents' needs with gradual reduction in the level of support), reflection (deliberately stimulating residents to think about their weaknesses and strengths in relation to the delivery of HV3C), or coaching (observing residents and providing concrete feedback on their 


\section{Pursuing High-Value, Cost-Conscious Care}

performance) could be interesting teaching methods. Yet these methods were not explicitly mentioned by participants. ${ }^{40}$ Although participants used the reported teaching methods deliberately, the intended lessons were often implicit and not verbally linked to HV3C. This finding resonates with previous research that revealed that residents struggle to identify and learn $\mathrm{HV} 3 \mathrm{C}$ in the workplace. ${ }^{16}$ Residents indicated needing the support of attending physicians to identify HV3C lessons and build competence. ${ }^{16}$ Improving HV3C training during residency seems therefore to depend on equipping attending physicians with a broader array of clinical teaching skills so that they can more effectively emphasize HV3C standards and decisions during supervision. From this perspective, the field of medical education has a role in providing attending physicians with practical examples of teaching methods that can support the specific learning of $\mathrm{HV} 3 \mathrm{C}$ in the workplace.

Although attending physicians are key figures during residency ${ }^{16,18}$ as role models and in creating a supportive environment for HV3C delivery, ${ }^{14-17,39}$ participants in our study voiced how they experienced obstacles to both delivering and demonstrating HV3C. In particular, two obstacles were mentioned: their reluctance to discuss HV3C with patients and their lack of knowledge of health care economics in general. The increased awareness regarding the need for sustainable and affordable care is not limited to the medical field ${ }^{7}$ but also incorporates patients and society and results in an expectation by patients to talk about health care costs with their physicians. ${ }^{41,42}$ Because it is known that conflicting role modeling behaviors are present in medical training ${ }^{15}$ and there is a correlation between practice patterns observed during residency and future care delivery, ${ }^{20,43}$ dealing with these obstacles is crucial for training and future health care. It is not surprising that today's attending physicians find it difficult to demonstrate and teach $\mathrm{HV} 3 \mathrm{C}$ delivery, considering that they themselves were not trained in these competencies ${ }^{13,16}$ and curricula focusing on stewardship, cost consciousness, and patientcentered care are only a recent addition to medical education. ${ }^{11}$ The absence of any mention of health care costs in the consultation room has been reported previously. ${ }^{17,44,45}$ Although this may be a delicate topic, it does provide a learning opportunity if residents can observe these conversations. ${ }^{46}$ Communication skills based on empathic conversation have been proposed to discuss health care costs with patients, yet they are rarely used. ${ }^{45,47}$ Recent research suggested supporting residents with access to data, ${ }^{17}$ yet this study demonstrates that not only residents but also attending physicians can be supported with feedback tools, such as benchmark data, referral data, costs of care, and patient satisfaction scores in their specialty. Attending physicians spoke positively about how this information helped them evaluate their own HV3C practice and their ability to train residents in such behavior. Structural use of such data could benefit both attending physicians and residents because reflective practice is known to be an important element of HV3C training ${ }^{14}$ and influences physician behavior. ${ }^{48}$ Feedback data also can help improve attending physicians understanding of health care economics. Nevertheless, it is advisable that data are not only accessible but openly discussed in a tolerant environment ${ }^{17,49}$ 
to prevent a judgmental setting.

Our results indicate that teaching HV3C is now an individual goal, based on personal beliefs and teaching methods that are considered helpful, with little or no feedback on care delivery or teaching. Therefore, we suggest that interventions focus not only on developing the teaching skills of attending physicians but also on developing a HV3C culture that both teaching staff and residents support. Our findings may stimulate a review by departmental chiefs and deans of the environment of attending physicians in light of teaching HV3C. Providing tools for reflective practice and knowledge transmission, together with a shared departmental goal in teaching and delivering HV3C, might be worth considering.

The study has limitations. Because of the attention currently given to HV3C, our participants might have given socially desirable answers. Our results also might have overestimated participants' attitudes toward HV3C. We tried to address this potential bias by asking participants to give specific examples to substantiate the attention given to $\mathrm{HV} 3 \mathrm{C}$ training in the clinical setting. For future research, it might be valuable to observe physician behavior in the workplace to enrich our understanding of how they train residents in HV3C and why ongoing training is challenging in this context. Our research drew from a deliberately chosen group of attending physicians who had been identified by their program directors, and this selection method might have caused unintended sampling bias. Our research aimed to describe how attending physicians prepare residents for the delivery of $\mathrm{HV} 3 \mathrm{C}$ in general.

Future research might explore the relationship between training approaches and physicians' specialty because known influencing factors such as patient-physician relationship, scale of institution, and ability to gain insight in practice patterns often differs between specialties. 16 Our sample $(\mathrm{N}=12)$ also contained few female participants $(\mathrm{n}=3)$. We do not know if overrepresentation of male participants influenced our findings; further exploration might be valuable.

\section{Conclusion}

How attending physicians deal with HV3C themselves influences how they prepare residents in the workplace. To optimize HV3C, it may be important to create an environment that supports attending physicians in $\mathrm{HV} 3 \mathrm{C}$ delivery and training by making $\mathrm{HV} 3 \mathrm{C}$ a shared goal, providing insight into practice behavior, and minimizing the influence of obstacles. Additionally, attending physicians could use support in the variety of teaching methods available to incorporate HV3C in daily teaching to stimulate continuous learning. 


\section{Pursuing High-Value, Cost-Conscious Care}

\section{References}

1. Hood VL, Weinberger SE. High value, cost-conscious care: An international imperative. Eur J Intern Med. 2012;23(6):495-498.

2. Berwick DM, Hackbarth AD. Eliminating waste in US health care. JAMA. 2012;307(14):1513-1516.

3. Weinberger SE. Providing high-value, cost-conscious care: A critical seventh general competency for physicians. Ann Intern Med. 2011;155(6):386-388.

4. Lucey CR. Medical education: Part of the problem and part of the solution. JAMA Intern Med. 2013;173(17):1639-1643.

5. Sklar DP. How medical education can add value to the health care delivery system. Acad Med. 2016;91(4):445-447.

6. Owens DK, Qaseem A, Chou R, Shekelle P; Clinical Guidelines Committee of the American College of Physicians. High-value, cost-conscious health care: Concepts for clinicians to evaluate the benefits, harms, and costs of medical interventions. Ann Intern Med. 2011;154(3):174-180.

7. Choosing Wisely. http://www.choosingwisely.org/. Published 2012. Accessed April 26, 2019.

8. Brody H. Medicine's ethical responsibility for health care reform - the Top Five list. N Engl J Med. 2010;362(4):283-285.

9. Williams D, Clancy C, Dine J; High Value Care Curriculum (HVC) 4.0. American College of Physicians. https://www.acponline.org/clinical-information/high-value-care/medical-educatorsresources/newly-revised-curriculum-for-educators-and-residents-version-40. Published 2012. Accessed March 5, 2018.

10. Havyer RD, Norby SM, Leep Hunderfund AN, et al. Science of health care delivery milestones for undergraduate medical education. BMC Med Educ. 2017;17(1):145.

11. Frank JR, Snell L, Sherbino J, eds. CanMEDS 2015 Physician Competency Framework. Ottawa, Ontario, Canada: Royal College of Physicians and Surgeons of Canada; 2015.

12. Cooke M. Cost consciousness in patient care-What is medical education's responsibility? N Engl J Med. 2010;362(14):1253-1255.

13. Wong BM, Holmboe ES. Transforming the academic faculty perspective in graduate Medical education to better align educational and clinical outcomes. Acad Med. 2016;91(4):473-479.

14. Stammen LA, Stalmeijer RE, Paternotte E, et al. Training physicians to provide high-value, costconscious care: A systematic review. JAMA. 2015;314(22):2384-2400.

15. Leep Hunderfund AN, Dyrbye LN, Starr SR, et al. Role modeling and regional health care intensity: U.S. medical student attitudes toward and experiences with cost-conscious care. Acad Med. 2017;92(5):694-702.

16. Stammen L, Slootweg I, Stalmeijer R, et al. The struggle is real: How residents learn to provide highvalue, cost-conscious care. Teach Learn Med. 2019;31:402-411.

17. Gupta R, Steers N, Moriates C, Wali S, Braddock CH III, Ong M. High-value care culture among the future physician workforce in internal medicine. Acad Med. 2019;94:1347-1354.

18. Eraut M, Alderton J, Cole G, Senker P. The development of knowledge and skills at work. In: Coffield F, ed. Differing Visions of a Learning Society: Research Findings. Vol 1. Bristol, England: Policy Press; 2000;231-262.

19. Eraut M. Informal learning in the workplace. Stud Cont Education. 2004;26:247-273.

20. Asch DA, Nicholson S, Srinivas S, Herrin J, Epstein AJ. Evaluating obstetrical residency programs using patient outcomes. JAMA. 2009;302(12):1277-1283.

21. Chen C, Petterson S, Phillips R, Bazemore A, Mullan F. Spending patterns in region of residency 
training and subsequent expenditures for care provided by practicing physicians for Medicare beneficiaries. JAMA. 2014;312(22):2385-2393.

22. Teunissen PW, Wilkinson, TJ. Learning and teaching in workplaces. In: Dornan T, Mann K, Scherpbier A, Spencer J, ed. Medical Education: Theory and Practice. London, England: Churchill Livingstone Elsevier; 2011.

23. Savin-Baden M, Howell Major C. Interviews. In: Savin-Baden M, Howell Major C. Qualitative research: the essential guide to theory and practice. Taylor and Francis Group, London and New York 2013;357-373.

24. Snijders M. ZonMw, high-value, cost-conscious care research. (Onderzoek naar doelmatigheid). The Netherlands Organisation for Health Research and Development. Published 2019. https://www.zonmw.nl/nl/onderzoek-resultaten/doelmatigheidsonderzoek/ Accessed May 2, 2019.

25. High-Value, Cost-Conscious Care Symposium; April 18, 2018; CORPUS Conference Centre, Leiden, Netherlands, https://www.bewustzijnsproject.nl/agenda-item/symposium2018/. Accessed March 10, 2018.

26. Noben C, Training Region, Southeast Netherlands (OORZON). High-value, cost-conscious care. (Doelmatigheid van zorg.) https://www.oorzon.nl. Published 2019. Accessed May 2, 2019.

27. Noben C, Habsati A, Brabant DB. High-value, cost-conscious care. (Doelmatigheid van zorg.) www. oorzon.nl/doelmatigheid.: OORZON; 2018. Accessed December 12, 2018.

28. Glaser BG, Strauss AL, Strutzel E. The discovery of grounded theory; Strategies for qualitative research. Nursing Research. 1968;17(4):364.

29. Charmaz K. Constructing Grounded Theory: A Practical Guide Through Qualitative Analysis. London, England: Sage Publications Ltd.; 2006.

30. Corbin J, Strauss A. Basics of Qualitative Research: Techniques and Procedures for Developing Grounded Theory. Thousand Oaks, CA: Sage Publications, Inc.; 2014.

31. Morse JM. The significance of saturation. Qualitative Health Research. May 1995:147-148.

32. Frambach JM, van der Vleuten CP, Durning SJ. AM last page. Quality criteria in qualitative and quantitative research. Acad Med. 2013;88(4):552.

33. Tong A, Sainsbury P, Craig J. Consolidated criteria for reporting qualitative research (COREQ): A 32-item checklist for interviews and focus groups. Int J Qual Health Care. 2007;19(6):349-357.

34. McMillan W. Theory in healthcare education research: The importance of worldview. In: Cleland J, Durning SJ, eds. Researching Medical Education. Chichester, West Sussex, England: John Wiley \& Sons; 2015;15-24.

35. Markee N. Emic and etic in qualitative research. In: The Encyclopedia of Applied Linguistics. Chichester, West Sussex, England: Wiley-Blackwell; 2013.

36. Carter K, Doyle W. Preconceptions in learning to teach. Educational Forum. 1995;59:186-195.

37. Levitt KE. An analysis of elementary teachers' beliefs regarding the teaching and learning of science. Science Education. 2002;86(1):1-22.

38. Richardson V. The role of attitudes and beliefs in learning to teach. In: Sikula JP, ed. Handbook of Research on Teacher Education: A Project of the Association of Teacher Educators. 2nd ed. New York, NY: MacMillan; 1996;102-119.

39. Sedrak MS, Patel MS, Ziemba JB, et al. Residents' self-report on why they order perceived unnecessary inpatient laboratory tests. J Hosp Med. 2016;11(12):869-872.

40. Stalmeijer RE. When I say... cognitive apprenticeship. Med Educ. 2015;49(4):355-356.

41. Danis M, Sommers R, Logan J, et al. Exploring public attitudes toward approaches to discussing costs in the clinical encounter. J Gen Intern Med. 2014;29(1):223-229.

42. Bullock AJ, Hofstatter EW, Yushak ML, Buss MK. Understanding patients' attitudes toward 


\section{Pursuing High-Value, Cost-Conscious Care}

communication about the cost of cancer care. J Oncol Pract. 2012;8(4):e50-e58.

43. Chen C, Petterson S, Phillips R, Bazemore A, Mullan F. Spending patterns in region of residency training and subsequent expenditures for care provided by practicing physicians for Medicare beneficiaries. JAMA. 2014;312(22):2385-2393.

44. King BC, DiPace J, Naifeh M, Hammad H, Gerber LM, Abramson E. Pediatric training faculty and resident perceptions on teaching high-value, cost-conscious care: A multi-institutional study. Hosp Pediatr. 2017;7(9):547-552.

45. Alexander GC, Casalino LP, Meltzer DO. Patient-physician communication about out-of-pocket costs. JAMA. 2003;290(7):953-958.

46. Tilburt JC, Wynia MK, Montori VM, et al. Shared decision-making as a cost-containment strategy: US physician reactions from a cross-sectional survey. BMJ Open. 2014;4(1):e004027.

47. Hardee JT, Platt FW, Kasper IK. Discussing health care costs with patients: An opportunity for empathic communication. J Gen Intern Med. 2005;20(7):666-669.

48. Mazmanian PE, Davis DA. Continuing medical education and the physician as a learner: Guide to the evidence. JAMA. 2002;288(9):1057-1060.

49. von Ferber L, Bausch J, Koster I, Schubert I, Ihle P. Pharmacotherapeutic circles. Results of an 18-month peer-review prescribing-improvement programme for general practitioners.

Pharmacoeconomics. 1999;16(3):273-283. 


\section{Appendix 4.I: Semi-structured interview}

Specialism, Date.

\section{Welcome:}

Brief explanation of semi-structured interview

Research goal: brief explanation of the framework and objective of the study

"Thank you for taking part in this semi-structured interview. At Maastricht University, we are conducting research into $\mathrm{HV} 3 \mathrm{C}$ and the role of education in this. I (LS) will lead this interview, I'm a family medicine resident. We're about to start but before we do, I'd like to say that our aim is to gain further insight into your experiences and opinions regarding the theme of HV3C, I'd like to emphasize that there are no right or wrong answers. Different experiences and opinions are all useful for our study. That's also why we'd like you to share information you feel is relevant."

\begin{tabular}{|c|c|c|c|}
\hline The questions & List of topics & $\begin{array}{l}\text { Theoretical } \\
\text { framework }\end{array}$ & $\begin{array}{l}\text { Time } \\
(\min )\end{array}$ \\
\hline $\begin{array}{l}\text { Would you like to introduce } \\
\text { yourself? }\end{array}$ & $\begin{array}{l}\text { - How are you involved in workplace-based learning? Other educational tasks. } \\
\text {-Years of experience as attending physician. } \\
\text { - Previous workplace(s), academic / peripheral. }\end{array}$ & & 5 \\
\hline What is HV3C? & $\begin{array}{l}\text {-What is the role of the patient in HV3C? } \\
\text {-What is the doctor's role? }\end{array}$ & & 10 \\
\hline $\begin{array}{l}\text { I) What do you think of } \\
\text { HV3C? }\end{array}$ & -What is the role of the organization? & & \\
\hline $\begin{array}{l}\text { Share definition of } \mathrm{HV} 3 \mathrm{C} \text { as } \\
\text { used by research team. }\end{array}$ & & & \\
\hline When do you come across & -Are residents involved in these examples, please elaborate? & & 10 \\
\hline $\mathrm{HV} 3 \mathrm{C}$ in daily practice? & $\begin{array}{l}\text {-What is the role of residents in these examples? } \\
\text { - If not? Is that a conscious choice? Why? }\end{array}$ & & \\
\hline $\begin{array}{l}\text { 2a) On a normal working } \\
\text { day, when would you be } \\
\text { giving } \mathrm{HV} 3 \mathrm{C} \text { ? }\end{array}$ & - Do you consider this a learning opportunity for residents? & & \\
\hline
\end{tabular}


How did you learn to deliver $3 \mathrm{~b}$ ) Where or from whom did you learn to deliver HV3C? (examples) Awareness HV3C?

3c) What made you aware of things associated with HV3C?

3a) What do you consider when you are concerned with HV3C?
In or outside the hospital / work-related or private. (examples)

3d) What information or knowledge do you need for HV3C delivery? *When protocols are mentioned, please discuss how decisions are made in absence of protocols.

What do you consider when you make a decision in light of HV3C?

- Patient preferences

- Knowledge of best practice / EBM

- Knowledge of costs / insurance

Where did you obtain that knowledge?

3e) Do you ever look back on /reflect on your actions from the standpoint of HV3C? (examples)

Reflection on action taken in practice

What triggered you to reflect on your actions in the light of HV3C? (examples)

3f) What role does $\mathrm{HV} 3 \mathrm{C}$ play in the department and during

Micro supervision moments? (examples)

Stimulating environment

3g) Have you seen differences between different hospitals? (examples) -Academic

- Peripheral

Stimulating environment

Where do you think those differences come from? (examples)
What do you need in your daily practice to prepare residents for $\mathrm{HV} 3 \mathrm{C}$ delivery?

-What is the residents' role in clinical teaching of HV3C? (examples)

What do you need to decide what kind of care would be considered $\mathrm{HV} 3 \mathrm{C}$ in daily practice?

Is there anything left that we haven't dealt with sufficiently?

What else do you think is important to share? 



\section{Chapter 5}

\section{A wealth of opportunities: an ethnographic study on learning to deliver high-value, cost-conscious \\ care}

Stammen, L.A., Janssen, L.M.E., Bressers, G., Driessen, E.W., Stassen, L.P.S., Stalmeijer, R.E., Scheele, F. (2019) A wealth of opportunities: an ethnographic study on learning to deliver high-value, cost-conscious care. MedRxiv. doi: https://doi.org//0.1 I0I//901 19/6/ Submitted for peer review. 


\section{Abstract}

Purpose: Since physicians' behavior determines up to $80 \%$ of total healthcare expenditures, training residents to deliver high-value, cost-conscious care is essential. Residents acknowledge the importance of high-value, cost-conscious care delivery, yet perceive training to be insufficient. We designed an observational study to gain insight into how the workplace setting relates to residents' high-value, cost-conscious care delivery.

Method: This ethnographic study builds on 175 hours of non-participant observations including informal interviews, nine semi-structured interviews and document analysis. Data was collected at the Department of Obstetrics and Gynecology in an academic hospital in the Netherlands and the sample included 21 gynecology residents. Data collection and analysis were iterative analyzing fieldnotes, interview transcripts and documents, through open coding, thematic analysis and axial analysis by a multidisciplinary research team.

Results: Residents rarely consider health care costs, and knowledge regarding costs is often absent. Attending physicians guide residents while balancing benefits, risks and costs, with or without explicating their decision-making process. Identified learning opportunities are elaboration on questions raised concerning high-value, cost-conscious care, checking information about costs that are used in discussions about high-value, cost-conscious care, and having a more open and explicit discussion about high-value, cost-conscious care.

Conclusion: Our study emphasizes that the opportunities and potential to train residents to deliver high-value, cost-conscious care in the workplace are present. The challenge resides in capitalizing on these opportunities. We suggest departments to consult external experts to facilitate discussions regarding high-value, cost-conscious care to contribute to informal learning and to create a workplace setting in which high-value, cost-conscious care delivery is prioritized. 


\section{Introduction}

Rising healthcare expenditure significantly impact the sustainability of healthcare systems. ${ }^{1-3}$ Limiting costs while ensuring the quality of care is therefore high on the political and medical agenda. ${ }^{4}$ Since physicians' behavior determines up to an estimated $80 \%$ of total healthcare expenditures, shaping the behavior of future physicians is a potentially valuable intervention for ensuring delivery of high-value, cost-conscious care (HV3C). ${ }^{5-9} \mathrm{HV} 3 \mathrm{C}$ is defined as 'care that aims to assess the benefits, harms, and costs of interventions' that leads to 'care that adds value' for the individual patient and the population in general. ${ }^{10} \mathrm{HV} 3 \mathrm{C}$ is characterized by three key principles: 1) it avoids wasteful care that has no added value for the patient, therewith reducing unnecessary costs; 2) it aligns with the personal preferences and needs of the patient; and 3) it is based on the latest evidence. ${ }^{2,10-12}$

Campaigns such as Choosing Wisely ${ }^{13}$ and Less is More ${ }^{14}$ have advanced awareness of unnecessary treatments and procedures and have led to a variety of initiatives, including educational interventions. High-profile organizations such as the Accreditation Council for Graduate Medical Education (ACGME), the Canadian Medical Education Directives for Specialists (CanMEDS), together with American College of Physicians (ACP) and General Medical Council (GMC) also aim to prepare residents for their future roles in HV3C delivery. ${ }^{15-17}$ A general aim of these organizations is to incorporate $\mathrm{HV} 3 \mathrm{C}$ in the training of physicians, residents and medical students. ${ }^{3,16}$ While initiatives mainly focused on formal education, ${ }^{8,18}$ residency training takes place in the clinical workplace where training is mostly informal. ${ }^{19}$

Workplace-based learning in the area of HV3C is increasingly investigated ${ }^{9,20}$ and results indicate that residents perceive $\mathrm{HV} 3 \mathrm{C}$ training to be insufficient. ${ }^{9}$ These findings are worrying, since workplace-based learning covers the majority of residency training ${ }^{21}$ and lessons from graduate medical education persist long into practice. ${ }^{22-24}$ We aimed to build on and expand this research by obtaining a real-life perspective on the workplace setting. Therefore, we designed an observational study to gain insight into how the workplace setting is related to residents' HV3-delivery. 


\section{Methods}

\section{Research design}

We conducted an ethnographic study to gain insight into how the workplace setting is related to residents' HV3C-delivery. Ethnography is a qualitative research methodology, commonly used to study cultures, by which data is collected through fieldwork. ${ }^{25,26}$ In this case fieldwork consisted of non-participatory observations, informal interviews, semi-structured interviews and document analysis to generate insight into the sample population's views, motives and actions in the real-life setting..$^{25,27}$ For a more elaborate description of ethnography as a methodology and insight in the iterative process of data collection and data analysis, we would like to refer to Appendix 5.1.

\section{Setting}

The fieldwork was conducted in an academic hospital in the Netherlands at a Department of Obstetrics and Gynecology. At the time of the study, a national government-subsidized project was running aimed at increasing awareness of $\mathrm{HV} 3 \mathrm{C}$ during residency training ${ }^{28}$. This project provided educational material and e-learning opportunities to stimulate residents to voluntarily set up projects to improve HV3C delivery in their department. During our data collection no formal education was present to teach residents HV3C-delivery.

\section{Subjects}

All 21 resident physicians working in the gynecology department at the time of our study participated. Prior to the study they were informed about the research by letter and attended a short presentation during the morning handover, in which we explained the primary research goal as 'we want to gain insight into how the workplace setting is related to residents' HV3Cdelivery'. Written informed consent was obtained from all participating residents. The Ethical Review Board of the Netherlands Association for Medical Education approved this study on August 24, 2017, under file number 881.

\section{Data collection}

The second author (LMJ) shadowed gynecology residents in daily practice for 175 hours during the period of three months. LMJ accompanied the residents during patient consultations, surgical procedures, deliveries and medical rounds, and attended meetings, e.g. 


\section{Pursuing High-Value, Cost-Conscious Care}

morning reports, educational activities and (multidisciplinary) seminars, as well as lunch and coffee breaks. These non-participant observations (i.e. the researcher was not involved in care delivery) focused on residents' behavior, conversations and situations regarding care delivery. Observations were followed by unstructured and ad hoc informal interviews with the aim of gaining thorough comprehension of the context and rationale of the observed moments. ${ }^{29,30}$ Additionally, semi-structured interviews were held (by LMJ and LAS) with nine purposively selected participants to gain additional information about specific observed situations and the department in general from representatives of the observed population. Participants were sampled based on the level of involvement in discussions and conversations regarding HV3C, both residents and staff members with high and low levels of involvement were selected. All interviews were audio-recorded and transcribed verbatim. Document analysis of hospital and national protocols and patient charts was executed to see, when residents or supervisors referred to protocols, whether their claims were supported as well as whether the decisionmaking process was retraceable.

Observed behavior and conversations were thickly described, written up in detailed, contextsensitive and locally informed fieldnotes about the observed events. ${ }^{25,31}$ Data collection involved iterative cycles of data collection and analysis, combining observations, interviews and analysis together with the research team (see Appendix 5.2). ${ }^{25,30}$ The iterative process of data collection discontinued when data saturation was reached, meaning data analysis did not result in new concepts and an adequate understanding of key concepts was gained. ${ }^{26}$ Data saturation was reached after approximately 175 hours of observation and nine semi-structured interviews.

\section{Data analysis}

As is common in ethnography, the data were analyzed iteratively (see Appendix 5.2) starting with line-by-line open coding by LMJ, LAS and GB. ${ }^{25,32}$ The next steps were thematic and axial analyses, in which we identified central themes and relationships between the various themes. As a result of team discussions, we gradually adapted the focus of the observational themes and conducted semi-structured interviews in order to deeper our understanding of the data. Additionally, FS checked if the observations represented national care delivery and not a specific regional setting. We are aware of the influence of the researchers' background on the process of data collection and data analysis, to increase transparency we included a reflexivity section in Appendix 5.1. 


\section{Results}

\section{The consideration of benefits, risks and health care costs in daily practice}

When protocols, guidelines or regional agreements failed to provide clear direction toward care delivery, or when new scientific information became accessible, health care professionals needed to decide which care delivery was considered 'appropriate'. Health care professionals then considered pros and cons, including concerns for patients, consequences of a missed diagnosis, respect for autonomy of patients, burden of testing or treatment, influence of quality of life and long-term health benefits. We rarely observed attention for health care costs, or conversations regarding who would eventually pay for those costs. In cases in which costs were included in the process of balancing pros and cons of health care services, the absence of knowledge regarding costs became apparent. Questions regarding financial costs arose when residents and attending physicians discussed a scientific article, reviewed the protocol, or discussed the necessity of structural testing. Observations demonstrated that financial reasoning did not influence the process of decision-making. We did not observe occasions in which health care costs were checked as a result of such discussions.

It is the end of the morning round and medical students, supervised by the resident-in-training are critically appraising a procedure.The resident noticed differences between the value and use of repetitive PCR-ratio (protein-to-creatine) determined in single voided urine versus ACR-ratio in 24-hour-collected urine for diagnosing pre-eclampsia $(P E)$ in their training region.

O I 3:Actually, this article shows that there is little difference between PCR (protein-to-creatine ratio) and ACR (24-hour albumin-to-creatinine ratio).

S3:And what about the cost?

A26:That's a good one, we should actually check that in the lab.

Document analysis demonstrated that costs were not checked nor was the protocol adapted as a result of the discussion. (Fieldnotes, February 21 , 2018)

The influence of the attending physician on the process of balancing benefits, risks and costs. HV3C-delivery by residents was guided by attending physicians' preference. In some cases, the attending physician extensively 'walked residents through' the process of balancing all relevant stakes that led to their proposed diagnostic or treatment plan. In other situations, the attending physician stated their opinion of what they considered to be appropriate care without further explanation. Although residents not always understood nor agreed with the attending physician, in general they did not request insight in the attending physicians' considerations regarding what was deemed appropriate. Residents tended to execute the advice of the attending physician with little countering of the consultants' judgment. 


\title{
Pursuing High-Value, Cost-Conscious Care
}

Variation between attending physicians was prominent and recognizable for residents and was sometimes considered confusing especially in the eyes of junior residents. Senior residents used these differences to get agreement on their own proposed plan by being deliberate in whose attending physician's advice they sought in certain cases, as mentioned by resident R17 in an informal interview.

\begin{abstract}
RI 7:'There are pretty big differences between the attending physicians. As a resident you have to get to know the bosses, so you know how they work.At some point you know the preferences of every senior physician, and if you have a certain plan in mind, you just call the attending physician that you think is likely to agree with your plan... Sometimes you think it is nonsense to do something and then you just need an attending physician who agrees with you.'
\end{abstract}

(Fieldnotes, February 22, 2018)

\section{Three observed learning opportunities regarding the process of balancing benefits,} risks and health care costs.

Based on the analysis of our observations, informal and formal interviews, it became apparent that there were three main areas for learning which could be improved. First, although residents and staff members raised questions regarding what constitutes high-value, cost-conscious care, we observed that questions regularly remained unanswered or undeliberated. In these situations, conversations were discontinued (for example by raising a different question) or paused ('we should check that'), without revisiting raised questions or checking/changing the course of action. Our observations also demonstrated questions followed by (collective) discussions, explicating the process of balancing benefits, risks and costs of care delivery. We asked both residents and supervisors how questions related to care delivery could be enhanced to facilitate residents' learning. Residents shared that they needed a more structural review of patient cases, followed by an organized overview of benefits, risks and costs of the case at hand. This could be done for example during the morning handover when discussing a patient case. Secondly, decisionmaking often was based on assumptions about costs without efforts to check them. This was observed for example when costs were considered 'high', yet actual amounts were not known or the meaning of commonly used terminology (for example what do we mean by 'bedrest') was not understood or defined. Thirdly, face-to-face conversations about care delivery were absent in instances where the line of reasoning was not clear for either staff or residents. Although attending physicians were sometimes openly critical to residents about how colleagues balanced benefits, risks and costs of care delivery, this did not result in a face-to-face conversation about the delivered care and consequently did not result in a take-home message for residents. For example, critiques often ended with comments such as "they probably had a good think about it" or "let's continue with the next case". Supervisors confirmed that especially formal meetings created interesting teaching opportunities but were hesitant to deepen the discussion in those meetings. Supervisors motivated this by describing how they aimed to maintain a safe learning environment (do not 
give the resident the impression their decision was 'false') while also exposing residents to the uncertainty that is present in care delivery (modeling that there is more than one 'correct' treatment decision).

\section{Discussion}

\section{Summarizing}

Training residents to provide $\mathrm{HV} 3 \mathrm{C}$ is essential for the sustainability of healthcare systems. ${ }^{2,5,9,10}$ This study explored how the workplace setting is related to the residents' HV3C-delivery. Our results demonstrated that costs are not structurally considered in the process of care delivery. Another finding was the strong influence of the attending physicians' opinion regarding what is considered high-value, cost-conscious care on the resident. Furthermore, learning opportunities regarding $\mathrm{HV} 3 \mathrm{C}$ were identified; elaboration on questions raised concerning $\mathrm{HV} 3 \mathrm{C}$, checking information about costs that are used in discussions about HV3C, and having a more open and explicit discussion about HV3C.

\section{Insight in relevant health care costs}

The importance of knowledge transmission for the training of high-value, cost-conscious care delivery has been emphasized in previous research. ${ }^{7,9}$ We observed that physicians aim to make health care costs part of the discussion, yet lack information to do so highlighting the importance of knowledge of health care costs in particular. Besides the lack of formal education, another known hurdle for knowledge transmission is the complexity of health care economics and the lack of transparency of costs in health care systems. ${ }^{33}$

\section{The consultant as leading role model}

The leading role of the attending physician in health care delivery in general, ${ }^{9,19,20}$ and HV3C specifically ${ }^{9,33}$ has been previously identified. Through observing role models' care delivery residents learn professional behaviors, particularly from those attending physicians that match their personal views. ${ }^{34}$ Data analysis demonstrates that senior residents intentionally seek advice from attending physicians who are likely to share their views on HV3C-delivery. Training opportunities lie in pursuing a workplace setting where attending physicians deliberately assist residents in explicating their reasoning regarding HV3C, with or without sharing the same view. This can be achieved by inviting residents to discuss, assess and reflect on future and past care delivery. Practice variations between consultants and departments could function as a starting point for these discussions and reflections, ${ }^{35,36}$ especially when residents express that they doubt if care delivery meets HV3C standards. 


\section{Pursuing High-Value, Cost-Conscious Care}

\section{Workplace culture}

Our results point to the absence of a culture that allows to openly question the line of reasoning of colleagues. Residents' and staff's tendency to favor agreement over critical discussions could explain this finding. Research findings indeed indicate that striving for harmony may interfere with the quality of postgraduate medical education and quality of care. ${ }^{37}$ Specifically, engaging in constructive discussions with attending physicians may be challenging in a workplace setting that is considered to be hierarchical. ${ }^{38}$ It is not surprising that the current workplace setting is not familiar with discussing care delivery from a HV3C perspective since issues of accessibility and affordably of health care are recent. Nevertheless, current budgetary crises in most health care systems urge for brisk culture change. To initiate a culture change, we suggest focusing interventions on attending physicians and other staff members. Changing the overarching culture and stimulating residents to actively engage in $\mathrm{HV} 3 \mathrm{C}$ is desirable. Therefore, future research should investigate how departments can be supported in developing a critical attitude toward care delivery in light of HV3C. Action research ${ }^{39}$ can be a suitable strategy to challenge conventional thinking about $\mathrm{HV} 3 \mathrm{C}$ in the clinical setting. In such design, integrating an independent external expert to regularly question care delivery, and guide group discussions in an open and tolerant manner, may speed up consciousness of value for patients, costs and the urge for bottom up system change. This 'devils' advocate' could help to fill in the opportunities detected in this study; elaboration on questions raised concerning HV3C, checking information about costs that is used in discussions about $\mathrm{HV} 3 \mathrm{C}$, and having a more open and explicit discussion about HV3C. The ultimate achievement would be that staff members and residents would take over this role and challenge each other to underpin their decision-making process in $\mathrm{HV} 3 \mathrm{C}$ or structurally reflect on HV3C-delivery via group discussions.

\section{Strengths \& limitations}

The main strength of this study is the use of ethnography to gain insight in how the workplace setting is related to residents' HV3C-delivery. A major strength of ethnography is the ability to observe things that those who are routinely involved may not see as well as collecting data that participant might not be willing to share with researchers. ${ }^{26}$ Another strength is the rigorous performance of our data collection and analysis, in compliance with quality criteria of qualitative research. Our study has several limitations. Participating residents might have been more favorable toward HV3C-delivery, as they were aware of the researchers' presence and the research goal, and observations might be an overestimation of the role of HV3C in residency training. A second limitation is the restricted scope of our ethnographic research, since the observations solely took place within one single specialty in one single hospital. We aimed to mitigate this limitation by providing a description of the setting in which the research was performed. 
Additionally, our findings were assessed by a member of our research team who is familiar with the context but not a member of it. Nevertheless, extrapolation to other residency programs or departments must be done prudently.

\section{Conclusions}

Our study emphasizes that the opportunities and potential to train residents to deliver highvalue, cost-conscious care in the workplace are present. The challenge resides in capitalizing on these opportunities. We suggest departments to consult external experts to facilitate discussions regarding high-value, cost-conscious care to contribute to informal learning and to create a workplace setting in which high-value, cost-conscious care delivery is prioritized.

\section{References}

1. Jeurissen P, Maarse H, Tanke M. Betaalbare zorg. Vol 1. Den Haag: SDU; 2018.

2. Berwick DM, Hackbarth AD. Eliminating waste in US health care. JAMA. 2012;307(14):1513-1516.

3. Holmboe E. ACGME milestones framework. https://www.acgme.org. Published 2015. Accessed2019.

4. Blumenthal D. Controlling health care expenditures. N Engl J Med. 2001;344(10):766-769.

5. Cooke M. Cost consciousness in patient care--what is medical education's responsibility? NEnglJ Med. 2010;362(14):1253-1255.

6. McDaniel CE, White AA, Bradford MC, et al. The High-Value Care Rounding Tool: Development and Validity Evidence. Acad Med. 2018;93(2):199-206.

7. Stammen LA, Stalmeijer RE, Paternotte E, et al. Training Physicians to Provide High-Value, CostConscious Care: A Systematic Review. JAMA. 2015;314(22):2384-2400.

8. Weinberger SE. Educating trainees about appropriate and cost-conscious diagnostic testing. Acad Med. 2011;86(11):1352.

9. Stammen LA, Slootweg I, Stalmeijer R, et al. The Struggle Is Real: How Residents Learn to Provide High-Value, Cost-Conscious Care. Teach Learn Med. 2019:1-10.

10. Owens DK, Qaseem A, Chou R, Shekelle P, Clinical Guidelines Committee of the American College of P. High-value, cost-conscious health care: concepts for clinicians to evaluate the benefits, harms, and costs of medical interventions. Ann Intern Med. 2011;154(3):174-180.

11. Horwitz LI, Masica AL, Auerbach AD. Introducing Choosing Wisely(R): Next steps in improving healthcare value. J Hosp Med. 2015;10(3):187-189.

12. Qaseem A, Alguire P, Dallas P, et al. Appropriate use of screening and diagnostic tests to foster highvalue, cost-conscious care. Ann Intern Med. 2012;156(2):147-149.

13. Levinson W, Born K, Wolfson D. Choosing Wisely Campaigns: A Work in Progress. JAMA. 2018;319(19):1975-1976.

14. Otte J. Less is More Medicine. ww.lessismoremedicine.com/. Published 2012. Accessed 11/07/2019.

15. Council GM. Outcomes for graduates. General Medical Counsil. Published 2018. Accessed 11/07/2019, 2019. 


\section{Pursuing High-Value, Cost-Conscious Care}

16. Frank JR SL, Sherbino J,. CanMEDS-framework. Royal college of physicians and surgeons of Canada;2015.

17. Caitlin Clancy M, C. Jessica Dine, Donna Williams, Cynthia D. Smith. High value care curriculum 4.0. https:/www.acponline.org/clinical-information/high-value-care/medical-educators-resources/ newly-revised-curriculum-for-educators-and-residents-version-40. Published 2019. Accessed 11/07/2019, 2019.

18. Ryskina KL, Dine CJ, Kim EJ, Bishop TF, Epstein AJ. Effect of Attending Practice Style on Generic Medication Prescribing by Residents in the Clinic Setting: An Observational Study. J Gen Intern Med. 2015;30(9):1286-1293.

19. Morris CB. Understanding medical education: evidence, theory and practice: the association for the study of medical education. 2010.

20. Stammen L. How do attending physicians prepare residents to deliver high-value, cost-conscious care? AcadMed. 2019.

21. Dornan T, Mann KV, Scherpbier AJ, Spencer JA. Medical Education: Theory and Practice E-Book. Elsevier Health Sciences; 2011.

22. Asch DA, Nicholson S, Srinivas S, Herrin J, Epstein AJ. Evaluating obstetrical residency programs using patient outcomes. JAMA. 2009;302(12):1277-1283.

23. Chen C, Petterson S, Phillips R, Bazemore A, Mullan F. Spending patterns in region of residency training and subsequent expenditures for care provided by practicing physicians for Medicare beneficiaries. JAMA. 2014;312(22):2385-2393.

24. Wong BM, Holmboe ES. Transforming the Academic Faculty Perspective in Graduate Medical Education to Better Align Educational and Clinical Outcomes. Acad Med. 2016;91(4):473-479.

25. Reeves S, Kuper A, Hodges BD. Qualitative research methodologies: ethnography. BMJ. 2008;337:a1020.

26. Savin-Baden M, Major C-H. Qualititaive Research: The Essential Guide to Theory and Practice. Qualitative Research: The Essential Guide to Theory and Practice Routledge. 2013.

27. Atkinson P, Pugsley L. Making sense of ethnography and medical education. Med Educ. 2005;39(2):228-234.

28. Bewustzijnsproject. Bewustzijnsproject. https://www.bewustzijnsproject.nl. Published 2015. Accessed 11/07/2019, 2019.

29. Reulink N, Lindeman L. Kwalitatief onderzoek. Participerende observatie, interviewen. 2005.

30. Whitehead TL. Basic classical ethnographic research methods. Cultural ecology of health and change. 2005;1:1-29.

31. Geertz C. Thick description: Toward an interpretive theory of culture. In: The cultural geography reader. Routledge; 2008:41-51.

32. Varpio L, Ajjawi R, Monrouxe LV, O’Brien BC, Rees CE. Shedding the cobra effect: problematising thematic emergence, triangulation, saturation and member checking. Med Educ. 2017;51(1):40-50.

33. Stammen LA, Driessen EW, Notermans CC, Scheele F, Stassen LP, Stalmeijer RE. How Do Attending Physicians Prepare Residents to Deliver High-Value, Cost-Conscious Care? Academic Medicine. 2019.

34. Jochemsen-van der Leeuw HR, van Dijk N, van Etten-Jamaludin FS, Wieringa-de Waard M. The attributes of the clinical trainer as a role model: a systematic review. Academic Medicine. 2013;88(1):26-34.

35. Holmboe E, Ginsburg S, Bernabeo E. The rotational approach to medical education: time to confront our assumptions? Med Educ. 2011;45(1):69-80. 
36. Djulbegovic B, Hamm RM, Mayrhofer T, Hozo I, Van den Ende J. Rationality, practice variation and person-centred health policy: a threshold hypothesis. J Eval Clin Pract. 2015;21(6):1121-1124.

37. Janss R, Rispens S, Segers M, Jehn KA. What is happening under the surface? Power, conflict and the performance of medical teams. Med Educ. 2012;46(9):838-849.

38. Janss R, Rispens S, Segers M, Jehn KA. What is happening under the surface? Power, conflict and the performance of medical teams. Medical education. 2012;46(9):838-849.

39. Lingard L, Albert M, Levinson W. Grounded theory, mixed methods, and action research. Bmj. 2008;337:a567. 


\section{Pursuing High-Value, Cost-Conscious Care}

\section{Appendix 5.I: Methods}

\section{Additional information}

\section{Ethnography as a methodology}

Ethnography is a research approach to study of people (behavior), cultures, and values, aiming to understand those being studied. Ethnography requires fieldwork to gain a detailed and comprehensive view of the group and its setting. The focus lies on everyday practice instead of unusual or peculiar events. In order to get an expression of what is 'normal' within a setting, researchers need to immerse and engage the setting of interest. A major strength of ethnography is the ability to observe things that those who are routinely involved may not see as well as collecting data that participant might not be willing to share with researchers.

\section{Data collection}

The second author (LMJ) shadowed gynecology residents in daily practice during three months in 2018. LMJ accompanied the residents during patient consultations, operations, deliveries and medical rounds, and attended meetings, e.g. morning reports, educational activities and (multidisciplinary) seminars, as well as lunch and coffee breaks. These non-participant observations focused on residents' behavior, conversations and situations regarding care delivery. Observations were followed by unstructured and ad hoc informal interviews with the aim to gain more comprehension of the background and rationale of the observed moments. ${ }^{1,2}$ Additionally, semi-structured interviews were held (by LMJ and LAS) with nine purposively selected participants, to gain additional information about specific observed situations and the department in general from representatives of the observed population. Participants were sampled based on the level of involvement in discussions and conversations regarding HV3C, we sampled both residents and staff members with high and low levels of involvement. Interview guides with discussion topics based on the data analysis were prepared beforehand. All interviews were audiorecorded were transcribed verbatim. Document analysis of hospital and national protocols and patient charts were executed to see, when residents or supervisors referred to protocols, whether their claims were supported and whether the decision-making process was retraceable.

Observed behavior and conversations were thickly described, written up in detailed, contextsensitive and locally informed fieldnotes about the observed events. ${ }^{3,4}$ These fieldnotes were written up on a daily base and fully transcribed within 24 hours. All participant-specific information was saved in a coded file so participants could be identified after anonymization. Data collection involved iterative cycles, combining observations, interviews and analysis together with the research team., As part of these iterative cycles the informal and semistructured interviews were used to further understand observations and reflect with senior consultants and residents on the preliminary findings regarding the learning process. The iterative 
process of data collection and analysis discontinued when data saturation was reached, meaning data analysis did not result in new concepts and an adequate understanding of key concepts was gained (see Appendix 5.2). ${ }^{3,5}$ Data saturation was reached after approximately 175 hours of observation and nine semi-structured interviews.

\section{Data analysis}

As is common in ethnography, the data were analyzed iteratively (see Appendix 5.2) starting with line-by-line open coding by LMJ, LAS and GB. ${ }^{3,6}$ The next steps were thematic and axial analyses, in which we identified central themes and relationships between the various themes. The research team discussed verbatim transcripts as well as the thematic and axial analyses of the transcripts. As a result, we gradually adapted the focus of the observational themes and to gain even more understanding of the data we conducted semi-structured interviews. Additionally, FS checked if the observations represented national care delivery and not a specific regional setting.

\section{Reflexivity}

Ethnography relies on the researcher to act as the primary research instrument, and therefore, we understand that the autobiography of the researcher shapes the observational experience and the process of data analysis. ${ }^{3,7}$ The research team consisted of; LAS, a PhD-candidate who combines research on how to train physicians to deliver HV3C with family medicine residency training; LMJ, a graduate year medical student who just completed her final clinical internship at the obstetrics and gynecology department; GB, a PhD-candidate and medical anthropologist and sociologist; EWD, a full professor in medical education; LPS, a surgeon with special expertise in residency training, RES, an educationalist with extensive experience in qualitative research in medical education and FS, a gynecologist and full professor in health systems innovation and education. During data collection and analysis, a reflective diary was kept, which was the basis for regular discussions with LAS, LMJ, GB and RES. LMJ was present at the observed department for a total of 6 months (both clinical internship and research). LAS has combined residency training with studying postgraduate medical education for the past 4 years. Therefore, regular discussions with LAS, LMJ, GB and RES focused on; the level of involvement in the subject culture, the respect to individuals and groups, the researchers' notion on the complexity of care and the different stakes that residents need to combine (patient care, time management, personal development, working within a team). We discussed these different topics in relation to the observed data which specific attention for influence on the process of data analysis. The different backgrounds of the research team were used to review the data from different perspectives, deepening the discussions about, and assumptions regarding the observed behavior. 


\section{References}

1. Reulink N, Lindeman L. Kwalitatief onderzoek. Participerende observatie, interviewen. 2005.

2. Whitehead TL. Basic classical ethnographic research methods. Cultural ecology of health and change. 2005;1:1-29.

3. Reeves S, Kuper A, Hodges BD. Qualitative research methodologies: ethnography. BMJ. 2008;337:a1020.

4. Geertz C. Thick description: Toward an interpretive theory of culture. In: The cultural geography reader. Routledge; 2008:41-51.

5. Atkinson P, Pugsley L. Making sense of ethnography and medical education. Med Educ. 2005;39(2):228-234.

6. Varpio L, Ajjawi R, Monrouxe LV, O'Brien BC, Rees CE. Shedding the cobra effect: problematising thematic emergence, triangulation, saturation and member checking. Med Educ. 2017;51(1):40-50.

7. Pope C. Conducting ethnography in medical settings. Med Educ. 2005;39(12):1180-1187. 
Appendix 5.2: The process of data collection and data analysis

Events in chronological order

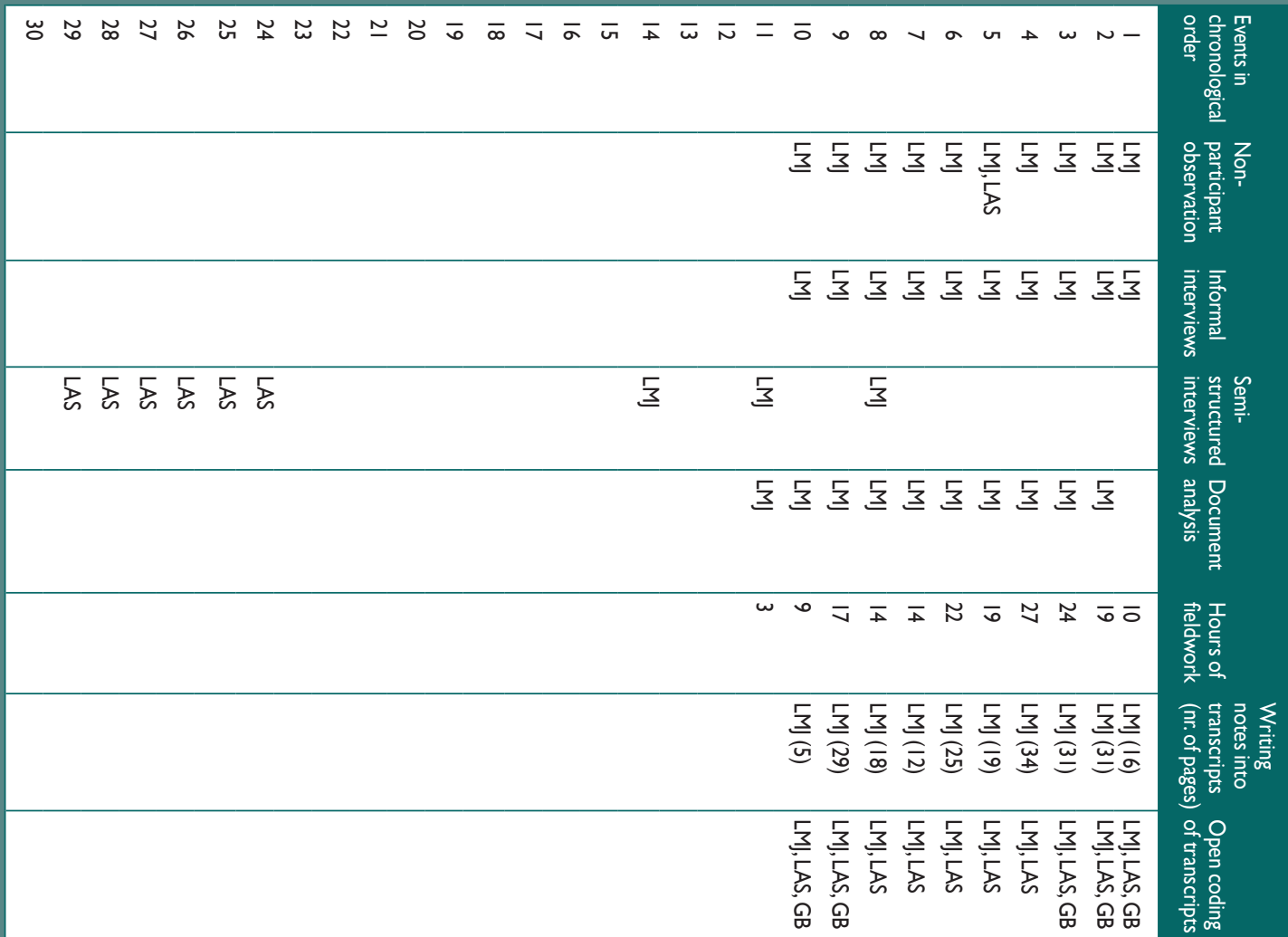

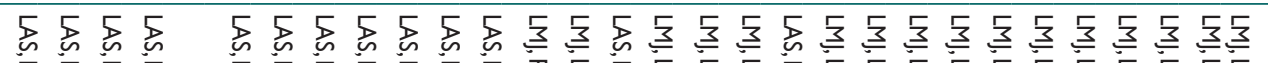

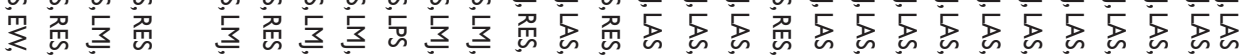

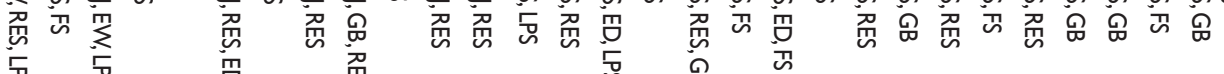

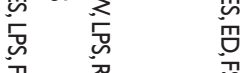
in 要 品

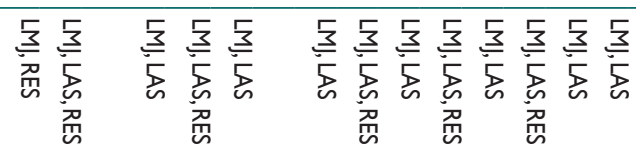

\begin{tabular}{|c|c|}
\hline 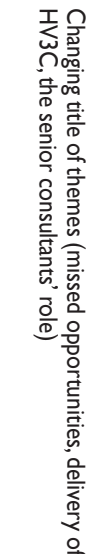 & 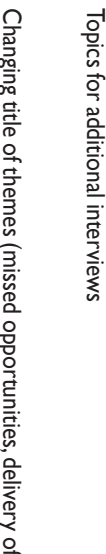 \\
\hline
\end{tabular}

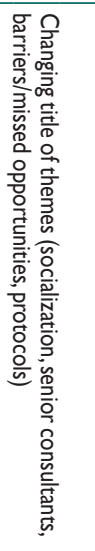

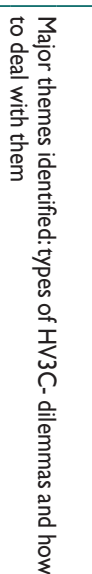

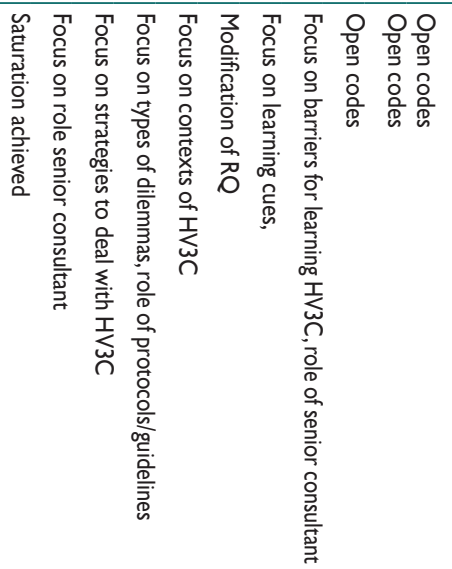




\section{Chapter 6}

12Tips voor het opleiden in doelmatigheid van zorg 


\section{2 tips voor het opleiden in Doelmatige Zorg}

Het is zaterdagmiddag en uw ontvangt als achterwacht een telefoontje van een 2de jaars AIOS
interne geneeskunde. Een 72-jarige patiënt heeft zich op de SEH gemeld met aanhoudende diarree
sinds 3 dagen. Directe reden om zich te melden is de geplande hartkatheterisatie aanstaande
maandag. De AIOS heeft de anamnese afgenomen en lichamelijk onderzoek uitgevoerd en belt u nu
om het vervolg beleid te bespreken. De bloedafname is bij binnenkomst op de SEH al gedaan
(pakket buikklachten, CRP I2, verder gb). Zijn samenvatting is; 72-jarige man met dreigende
dehydratie zonder koorts WD: virale gastro-enteritis. Zijn voorstel; opname ter observatie, 2.OL
infuus, afname feceskweken.

Het opleiden van arts-assistenten in het leveren van doelmatige zorg krijgt steeds meer aandacht. Doelmatige zorg is het afwegen van voordelen, nadelen en kosten van onderzoeken en behandelingen, om op die manier zorg te vermijden welke weinig of geen toegevoegde waarde heeft voor de patiënt. Doelmatige zorg gaat zowel over het doen van dat wat nodig is als het vermijden van dat wat weinig waarde heeft. Behoudens aanvullend onderzoek, beeldvorming, en behandeling, heeft het ook betrekking op de frequentie van controles en het selecteren van de meest gepaste zorgverlener. Aangezien een groot deel van de opleiding plaatsvindt binnen de muren van gezondheidsinstellingen, praktijken en ziekenhuizen, willen wij in dit artikel 12 tips aanbieden om het werkplekleren te ondersteunen.

\section{Tip I Ben u bewust van uw eigen ten aanzien van doelmatige zorg. ${ }^{(1,2)}$}

Uw eigen opvattingen over doelmatige zorg hebben grote invloed op of $\mathrm{u}$ aandacht besteedt aan doelmatigheid. Vragen die u zichzelf kunt stellen zijn: Hoe staat u ten opzichte van doelmatige zorg en welke taak ziet $\mathrm{u}$ voor $\mathrm{u}$ zelf binnen doelmatigheid van zorg? Wie is in Nederland verantwoordelijk voor het zinnig gebruik van de zorg, en welke andere spelers (overheid, zorgverzekeraars, rechtelijke macht, burgers) spelen hierbij een rol? In het begeleiden van AIOS is het ook raadzaam deze ideeën over doelmatige zorg met AIOS te bespreken, en AIOS te stimuleren over hun verantwoordelijkheden ten aanzien van toegankelijkheid en betaalbaarheid na te denken. Sommige AIOS zijn continu bezig met thema's zoals over-diagnostiek, verspilling, en patiënt-voorkeuren en bestempelen zichzelf als 'doelmatige dokters' terwijl anderen twijfelen óf er überhaupt een rol weggelegd is voor dokters in het toegankelijk en betaalbaar houden van de zorg. Het besteden van aandacht aan het thema doelmatigheid in de praktijk wordt bemoeilijkt door concurrerende leerdoelen en tijdgebrek. AIOS zullen in de praktijk, indien de mogelijkheid bestaat, advies in winnen bij supervisoren welke op basis van eerdere ervaringen waarschijnlijk akkoord zullen gaan met hun voorgestelde beleid. Dit zijn vaak begeleiders die soortgelijke opvattingen hebben ten aanzien van welke zorg 'zinvol' is. 
Tip 2 Stel kritische vragen over doelmatigheid.(')

Kritische vragen stellen is een veelgebruikte techniek om het doelmatig denken op de werkplek te stimuleren. 'Welke andere beeldvormende technieken heb je overwogen?' en 'wat ga je doen bij een twijfelachtige uitslag?', 'wat zijn de voordelen en risico's van een afwachtend beleid?' zijn veel voorkomende vragen om AIOS te stimuleren om na te denken over het nut of de noodzaak van aanvullend onderzoek en inzicht te krijgen in de lijn van redeneren. Belangrijk is dat er ruimte is om de redenering te bespreken in een specifieke casus waarbij context factoren het beleidsvoorstel beïnvloeden. Supervisoren kunnen dit proces stimuleren door zelf, gevraagd of ongevraagd, hun redenatie te delen en aan te geven waarom ze bij hun patiënt bepaalde keuzes hebben gemaakt.

Tip 3 Wees u bewust van uw functie als rolmodel voor het leveren van doelmatige zorg. ${ }^{(1-3)}$ AIOS leren in de dagelijkse praktijk door zorg te leveren en te leren van hun ervaringen. Daarnaast zien ze dagelijks u als supervisor zorg leveren. Het is belangrijk te beseffen dat hoe de huidige specialisten omgaan met doelmatigheid een grote invloed heeft op hoeveel en wat AIOS leren over dit onderwerp. AIOS die worden opgeleid in een vakgroep waarbij de heersende opinie is dat doelmatigheid niet de verantwoordelijkheid van de artsen is, zal veel lastiger kritisch nadenken over zijn of haar eigen rol binnen doelmatigheid. Daarnaast kunnen AIOS veel leren van supervisoren die in hun klinisch redeneren het belang van patiënt en maatschappij meewegen.

\section{Tip 4 Geef AIOS de ruimte voor het vervolgen van eigen beleidsvoorstellen. ${ }^{(2)}$}

AIOS geven aan veel te leren van supervisoren waarbij ze, binnen de kaders van patiënt-veiligheid, hun eigen beleidsvoorstel mogen vervolgen. Supervisoren kunnen aangeven dat ze het zelf anders zouden doen, en toelichten waarom ze het anders doen, en toch de AIOS de vrijheid geven hun voorstel te vervolgen. In bovenstaande casus is het bijvoorbeeld maar zeer de vraag of feceskweken gaan bijdragen aan het herstel van deze patiënt. Het is leerzaam om nadien samen met de AIOS terug te kijken wat de voor en nadelen van het gekozen beleid zijn, en wat er geleerd is van de casus. Hierbij hoort ook het bespreken van verklaarde (op basis van context) en onverklaarde (op basis van willekeur) praktijkvariatie.

\section{Tip 5 Betrek AIOS in discussies rondom complexe casuïstiek. ${ }^{(2)}$}

Op de werkvloer komen regelmatig situaties voor waarbij er veel discussie mogelijk is. Denk daarbij bijvoorbeeld aan het uitvoeren van een complexe operatie bij iemand in slechte conditie of een patiënt die een bepaalde behandeling wil. Voor het leerproces van de AIOS is het van belang dat zij mee kunnen praten in discussies, ook als deze multidisciplinair van aard zijn. Sommige AIOS ervaren dat hun inbreng niet gewaardeerd word t of weten zelf niet goed of ze in de positie zijn om hun mening te delen. 


\section{Tip 6 Stimuleer AIOS om advies in te winnen buiten de vakgroep. ${ }^{(2,4)}$}

Mensen uit een ander discipline kunnen vaak belangrijke inzichten geven ten aanzien van welke zorg gepast is voor een patiënt. Denk bijvoorbeeld aan het bellen van een fysiotherapeut in het kader van revalidatie, het bellen van een klinisch chemicus over de interpretatie van een bloeduitslag, of maatschappelijk werk over het netwerk van de patiënt. Op de werkplek kunnen AIOS veel leren van andere beroepsgroepen of specialisaties als er onderling contact is. Dit lukt vaak makkelijker binnen kleinere organisaties of ziekenhuizen, maar is juist ook waardevol binnen een grotere organisatie. Voor supervisoren is het van belang deze externe expertise te erkennen en AIOS te stimuleren contacten te leggen buiten de vakgroep.

\section{Tip 7 Vraag/zoek spiegel-informatie en gebruik deze voor reflectie. ${ }^{(1,4)}$}

In sommige domeinen is er informatie voorhanden welke inzicht geeft in het aantal verwijzingen, aanvraaggedrag, voorschrijfgedrag of het optreden van complicaties. Deze data kunnen, mits op de juiste manier gepresenteerd en geïnterpreteerd, inzicht geven in het functioneren van het team of individu. Ze zijn het startpunt voor reflectie op het eigen handelen, maar moeten we aan een aantal basisvoorwaarden voldoen. Deze basisvoorwaarden voor een zinvolle kritisch reflectie is de beschikking over achtergrondkennis, eventuele verschillen tussen aanvragers, adequate dossiervoering en een veilige omgeving waarin het doel kwaliteitsverbetering is. Valkuilen zijn aannames dat: 1) minder aanvragen beter is, 2) het doel kostenbesparing is, of dat 3) praktijkvariatie onwenselijk is. Er kan gekozen worden voor een collectieve format zodat AIOS en supervisoren gezamenlijk inzicht krijgen in hun eigen handelen, of voor een format waarbij er kleine groepen worden gemaakt, gebaseerd op opleidingsjaar of aandachtsgebied. Belangrijke overwegingen hierbij met name de mate van het creëren van veilige omgeving, het belang van transparantie en de toegang tot medische expertise.

\section{Tip 8 Maak variatie tussen collega's bespreekbaar. ${ }^{(2,3)}$}

Als medische professional is het van belang om kritisch te kunnen kijken naar het eigen werk en het werk van collega's. Er is waarschijnlijk geen AIOS of specialist welke zal ontkennen dat er verschillen zijn tussen dokters. We maken hierbij onderscheid tussen 'gewenste praktijkvariatie' en 'ongewenste praktijkvariatie'. De grenzen tussen deze twee soorten praktijkvariatie zijn op papier eenvoudig, gewenst is alles wat valt onder de noemer 'meerdere wegen die naar Rome leiden' en ongewenst is 'zorg waarvan bekend is dat het ongepast of schadelijk is, of zorg 'die niet verklaard kan worden door de context van de situatie'. Echter, in de praktijk kunnen deze twee soorten dicht bij elkaar liggen en is het voor AIOS niet altijd duidelijk of praktijk variatie gewenst of ongewenst is. Bespreek met AIOS welke variatie ze zien, en probeer door middel van een gesprek te achterhalen 1) of men het onderscheid kan maken en 2) op basis waarvan de AIOS het onderscheid maakt. Mocht u een stapje verder willen, dat zou ook het bespreekbaar maken van variatie en het omgaan met verschillende supervisoren een onderwerp van gesprek kunnen zijn. 


\section{Pursuing High-Value, Cost-Conscious Care}

Tip 9 Bespreek altijd expliciet de hulpvraag van de patiënt. ${ }^{(3,4)}$

Het formuleren van een hulpvraag door de patiënt, eventueel met hulp van de AIOS, is essentieel om zorg te leveren welke 'waardevol' is. Helaas blijkt in de hectiek van de dag vaak de klacht 'patiënt heeft diarree' een meer prominente rol te spelen dan een eventuele hulpvraag 'patiënt wil voorkomen dat de geplande hartkatheterisatie geannuleerd wordt'. Het is voor supervisoren belangrijk om AIOS te stimuleren inzicht te krijgen in de voorkeuren, verwachtingen en wensen van de patiënt. Sommige verwachten van patiënten zijn echter niet erg realistisch bijvoorbeeld 'ik wil zekerheid dat ik geen kanker heb' en het is dan ook van groot belang dat, liefst in een vroeg stadium, er met patiënten gesproken wordt over de mogelijkheden en grenzen van de zorg.

\section{Tip 10 Let's talk about money. ${ }^{(1,2,4)}$}

Maak geld bespreekbaar, zowel in uw eigen (observeerbare) consultaties als tijdens overdrachten, supervisie-momenten en vergaderingen. Denk bijvoorbeeld over het spreken over een eigen risico of eigen bijdrage voor patiënt, wie opdraaft voor zorgkosten van onverzekerde patiënten en financiële verschillen tussen behandelingen. Er zijn hierbij 2 belangrijke struikelblokken; 1) veel van deze kennis ontbreekt bij AIOS en medisch specialist en is lastig te verkrijgen voor artsen, 2) praten over geld lijkt haaks te op ons doel om 'de beste zorg voor de patiënt te leveren' en het behouden van een vertrouwensrelatie met de patiënt. Toegang tot kennis over kosten (zorgverzekeringen, betalingsstelsel, financiële structuur van ziekenhuis met MSB of loondienstconstructie) is niet altijd voorhanden, echter er zijn een aantal cursussen voor specialisten en AIOS beschikbaar of een medisch manager kan gevraagd worden inzicht te geven in de financiële situatie van een afdeling. Daarnaast is het ook wenselijk om, met name de ouderejaars AIOS, openheid van zaken te geven ten aanzien van financiële stromingen binnen de vakgroep. Het is een vaardigheid om kosten (voor patiënt en maatschappij) met patiënten te bespreken zonder de arts-patiënt relatie schade toe te brengen. De kwaliteit van de Nederlands zorg staat hoog aangeschreven en gelukkig zijn kosten niet leidend in het maken van beslissingen in de zorg. Aan de andere kant is het van algemeen belang dat we de zorg betaalbaar en toegankelijk houden voor alle Nederlanders. Artsen die ervaring hebben met het bespreken van kosten met patiënten erkennen het delicate karakter, maar geven aan dat patiënten het waarderen en het de arts-patiënt relatie verstevigd. Indien er collega's zijn die zich vertrouwd voelen met het bespreekbaar maken van kosten in de spreekkamer, kan een 'kijkje in andermans keuken' wellicht uitkomst bieden.

\section{Tip II Neem als vakgroep een collectief standpunt in.(2)}

Hoe gaan we om met eisende patiënten? Hoe streng houden we vast aan gestelde criteria? AIOS merken op de werkvloer dat er veel verschillen zijn rondom het interpreteren van afspraken of het omgaan met concurrentie tussen ziekenhuizen. Dit maakt het lastig om zich aan te passen en te reflecteren op doelmatige zorg. Een voorbeeld is het geven van een bloedtransfusies bij iemand met klachten en een grenswaarde-HB of het behandelen van de subklinische hypothyreoïdie. Ook zijn er situaties waarbij patiënten een bepaalde ondoelmatige behandeling eisen en bij het 
weigeren van het inwilligen van dergelijk verzoek een ander ziekenhuis of praktijk contacteren. AIOS kunnen in grote mate van zelfstandigheid met dergelijke casussen omgaan indien er een afdelingsbeleid is dat leiding geeft.

\section{Tip 12 Maak gebruik van onderwijskundige ondersteuning.(1,3)}

De banden tussen opleiders, opleidingen en onderwijskundige zijn vaak niet zichtbaar maar wel aanwezig. Indien u zichzelf beter wil bekwamen in het opleiden in doelmatigheid, zoek dan onderwijskundige ondersteuning. Dit kan in de vorm van docent professionalisering, externe expertise, of onderwijskundige literatuur.

\section{Referenties}

1. Stammen LA, Driessen EW, Notermans C, Scheele F, Stassen LPS, Stalmeijer RE. How Do Attending Physicians Prepare Residents to Deliver High-Value, Cost-Conscious Care? Acad Med. 2019.

2. Stammen L, Slootweg I, Stalmeijer R, Janssen L, Stassen L, Scheele F, et al. The Struggle Is Real: How Residents Learn to Provide High-Value, Cost-Conscious Care. Teach Learn Med. 2019;31(4):402-11.

3. Stammen LA, Janssen LM, Bressers G, Driessen EW, Stassen LP, Stalmeijer RE, et al. A wealth of opportunities: an ethnographic study on learning to deliver high-value, cost-conscious care. medRxiv. 2019:19011916.

4. Stammen LA, Stalmeijer RE, Paternotte E, Oudkerk Pool A, Driessen EW, Scheele F, et al. Training Physicians to Provide High-Value, Cost-Conscious Care: A Systematic Review. JAMA. 2015;314(22):2384-400. 




\section{Chapter 7 General Discussion}


Medical education has the potential to curb health care costs and enhance health care quality. To capitalize this potential, it is important to unravel how medical residents are currently trained, how training can be supported, and which factors hinder or facilitate learning to deliver highvalue, cost-conscious care (HV3C). Insight into these issues can help medical education to fulfill its task of training physicians for their role in sustainable health care.

Given the importance of high-value, cost-conscious care for the sustainability of health care and the essential role of (future) physicians in this endeavor, this dissertation investigates the role of medical education in the training of high-value, cost-conscious care delivery using the following research questions:

I. Which elements of educational interventions are effective to train (future) physicians in the delivery of high-value, cost-conscious care?

2. How do residents approach the delivery of high-value, cost-conscious care in daily practice?

3. How do supervisors aim to train residents in the delivery of high-value, cost-conscious care?

4. How is the workplace setting related to residents' high-value, cost-conscious care delivery?

\section{Which elements of educational interventions are effective to train (future) physicians in the delivery of high-value, cost-conscious care?}

\section{Knowledge transmission \& reflective practice}

Various educational interventions have been implemented as strategies to train physicians, residents, and medical students in HV3C-delivery. In Chapter 2 we explored which elements of educational interventions are effective in training (future) physicians. We concluded that two of the three important 'mechanisms of action' in these educational interventions are aimed at knowledge transmission and stimulating reflective practice. ${ }^{1}$ Knowledge transmission often contains information on health care costs or on the appropriate health care services for specific patient cases. Knowledge transmission can effectively improve knowledge of health care costs and general health care economics, knowledge regarding evidence-based medicine, and knowledge regarding patient preferences. The goal of knowledge transmission is to help trainees identify how different health care services relate to value, both considering the individual patient and the society as a whole. Reflective practice can be used to critically appraise delivered care, helping physicians to gain insight in behavioral patterns and practice variation. Nguyen et al.

I The third 'mechanism of action' is the supportive environment and will be discussed separately. 


\section{Pursuing High-Value, Cost-Conscious Care}

defined reflection as "attentive, critical, exploratory and iterative interactions" where the focus lies on understanding and changing one's thoughts and actions. ${ }^{2}$ Reflective practice is associated with deep learning and understanding ${ }^{3}$ and demands an open and tolerant manner, with trainees or physicians that can create a non-judgmental setting. ${ }^{4}$ In order to utilize reflective practice to train HV3C-delivery it is important that it does not focus on volume without taking case specific information into account in order to determine appropriateness.

\section{Supportive environment}

Although knowledge transmission and reflective practice are essential for the delivery of high-value, cost-conscious care, our research indicated that in order to reach educational 'interventions' full potential a supportive environment should be present. This supportive environment entails that the goals of HV3C-delivery should be shared within the workplace-based environment. In medical educational literature, the lessons learned through the process of socialization are described as a hidden curriculum. Although the hidden curriculum is a powerful determinant of learning in general, it is known to be extremely influential in transferring norms and values to the trainees. Therefore, educational interventions should focus on the culture present since education is far less effective without a supportive environment that validates formal lessons. How the workplace environment influences the learning of $\mathrm{HV} 3 \mathrm{C}$ will be described more extensively on page 151 .

\section{How do residents approach the delivery of high-value, cost-conscious care in daily practice?}

Our research identifies that residents can influence their training by engaging in valuable learning opportunities in daily practice. Their personal attitude to the concept of HV3C is an important factor that influences their learning. Chapter 3 describes a spectrum of different resident attitudes. The spectrum ranges from 'the frugal type', who is always concerned with $\mathrm{HV} 3 \mathrm{C}$, to the 'costs-are-not-on-my-mind' type, describing the resident who is 'not bothered' by financial concerns. In between, we identified a group with a more moderate attitude that can be described as mindful of HV3C but seems to find it difficult to prioritize it along with other tasks in daily practice. These residents are illustrated by this participant's quote: "It's our responsibility to deliver HV3C but..." Although the pressing crisis of health care costs might render it impossible for the 'not bothered' residents to maintain their stance, this study highlights the importance of accounting for residents' HV3C attitude during their training. For training purposes, it is important to identify if residents feel familiar with a certain approach and to deliberate with residents on how it influences their care delivery and training. Although we did not focus on why residents favored a certain approach, the literature describes how the values of the community 
influence the residents' values, leading to an increased interest in the supervisors' and other health care professionals' approaches. Communal values are part of the hidden curriculum that is welldescribed in the medical educational literature.

\section{How do supervisors aim to train residents in the delivery of high-value, cost-conscious care?}

Supervisors play an important role in the training of residents as they supervise, demonstrate, guide, advise, role model different skills, techniques, attitudes and 'knowledge', in the workplacebased environment (Chapter 4). In Chapter 4 we set out to identify how supervisors aim to train residents to deliver high-value, cost-conscious care.

\section{Supervisors' approach to teaching HV3C}

Similar to residents, supervisors also displayed idiosyncratic approaches to HV3C in their own care practices. These approaches influenced the extent to which supervisor prioritized HV3C in training their residents.

Although postgraduate medical training is more and more focused on faculty development of supervisors $^{5}$ (i.e. training physicians in their role as clinical trainers) supervisors do not seem to incorporate many different teaching methods for HV3C-training. Supervisors mentioned using three teaching methods for residency training in HV3C: Socratic questioning (critically questioning medical decisions), setting limits (especially for more junior residents, e.g. specifying which tests could be ordered) and role modeling. Especially role modeling is known to be a potent source of learning within the clinical workplace. ${ }^{6}$ As residents learn through participation in day-to-day clinical practice, observing their supervisors is an important learning mechanism. ${ }^{6}$ However, role modeled behavior can lead either to very positive learning outcomes or lasting negative outcomes. As such it is important that supervisors are aware of the HV3C behavior that they display to residents.

\section{Factors limiting supervisors' attention to HV3C during residency training}

Supervisors described their own lack of knowledge regarding health care costs as one of the main factors limiting their attention to $\mathrm{HV} 3 \mathrm{C}$ during residency training. This finding strongly resonates with current research pointing to knowledge of costs-deficits and issues regarding transparency and accessibility of price composition. ${ }^{7-9}$ Simultaneously, lack of feedback and feedback data on for example cost of care and test ordering behavior, hampered supervisors' reflection on their own $\mathrm{HV} 3 \mathrm{C}$ practice. 
Typically, supervisors described the challenge of discussing health care costs with patients, something which is often observed by residents. Residents are aware of the lack of comfort with cost-conversations and claim that over $20 \%$ of clinicians in their training environment feel that it is not their role to discuss costs of tests or treatment with patients. ${ }^{9}$

All in all, our findings highlight that to create a learning environment in which HV3C is supported, supervisors' approach, HV3C practice and supervisory and role modeling skills require attention.

\section{The influence of the workplace setting on high-value, cost-conscious care training.}

Health care professionals and support staff are part of the workplace setting and are consciously or unconsciously involved in teaching. Residents collaborate with professionals outside their own department and are exposed to various resources and organizational structures. Multiple studies in this dissertation underpin the importance of the support given to HV3C training present in the workplace setting. ${ }^{9}$

\section{Supportive organization}

An HV3C supportive environment is characterized by the presence of the goal to deliver HV3C shared by members of the workplace. Chapter 2 concluded that if an educational intervention focuses on formal education alone and therefore does not reflect a shared idea of how care delivery can be improved, its effect is limited. This supportive environment is essential for both knowledge transmission and reflective practice. Besides support from 'working staff, our review concluded that support on the macro level is also crucial. On this level, organizational structure was often perceived to frustrate the delivery of HV3C. It could be local, for example the pressure to adhere to guidelines ${ }^{10}$ or a focus on volume rather than quality, or national, for example health legislation that increased the fear of malpractice suits. ${ }^{11,12}$ Other organizational factors that might influence HV3C delivery are the bureaucratic structure, ${ }^{13,14}$ workload, ${ }^{13,15}$ availability of resources, ${ }^{16-18}$ and access to and transparency of health care costs. ${ }^{19,20}$ A survey of pediatric training faculty and residents indicated that the lack of hospital support was the largest barrier to teaching HV3C. ${ }^{5}$ Support in the residents' workplace setting through resources and expertise (Chapter 3) is vital for HV3C training. Small and middle-sized hospitals/institutions were more likely to create opportunities to use the available resources and expertise. Consequently, residents described receiving more feedback and background information regarding the appropriateness of clinical decisions and better inter- and intra-professional collaboration in these hospitals/ institutions. 


\section{Supportive training program}

The elements described above (approaches, teaching methods, dealing with obstacles) are all features of the environment in which residents work and train. Our results demonstrate that exposure to variation between health care institutions and departments (Chapter 3) can offer HV3C learning opportunities. Residents can use observed variation to critically assess care delivery in terms of HV3C. Some supervisors exposed residents deliberately to variation to educate residents about the complexity of health care. Some residents, however, were confused by variation, especially when there was no clear line of reasoning between different decisions. The training program could facilitate residents' learning by promoting involvement in decisionmaking. Residents said that they felt unsure if engaging in discussions on appropriateness in complex cases would be valued. Yet, where residents were involved, these situations were considered as important teaching moments for HV3C delivery, especially when the resident was given the opportunity to pursue their own proposed treatment plan. Uncertainty, ${ }^{21}$ fear of missing a diagnosis, ${ }^{22}$ and time pressure ${ }^{23}$ are known forces that hinder HV3C delivery and make residents struggle with HV3C. Discussing these topics, most often initiated by the supervisor, can help resident to reflect on which forces influenced their medical decision-making.

\section{Clinical learning opportunities}

In the workplace setting, opportunities lie in asking critical questions to unravel the line of reasoning and explore alternatives in diagnostic, treatment, and follow-up processes. Taking a critical attitude to what can be considered appropriate and balancing the pros and cons of services are valuable exercises for residents. We observed that clinical decision-making was often based on assumptions, regarding the guideline recommendation, the patient's preference, or the associated health care costs. Sometimes, these assumptions were transferred from one person to the other, without the recipient checking the background of the information shared, leading to wide-spread misconceptions regarding the motives for care delivery.

Our observations of residents (Chapter 5) made it clear that they were reluctant to express criticism of decisions made by colleagues (Chapter 6). Even when care delivery by colleagues required clarification, the friendly atmosphere made it difficult to exploit these conversations to their full potential, possibly in fear of offending others. Although critical comments were made, they rarely led to an open review of care delivery and consequently did not translate into valuable teaching moments for residents. 


\section{Pursuing High-Value, Cost-Conscious Care}

Table 8: Main results of this dissertation

\section{Effective educational Knowledge transmission}

interventions

\section{\& Reflective practice}

Conditions for knowledge transmission: Knowledge of patient preferences, knowledge of costs and general health economics, knowledge of scientific evidence.

Conditions for reflective practice:

Supportive environment

Conditions for supportive practice:

Role of resident Residents' attitude

Always on my mind (Frugal type)

Important but...

Not on my mind

Role of supervisor Supervisors' attitude determines Priority given to $\mathrm{HV} 3 \mathrm{C}$ training

Feedback on HV3C delivery

Perceived obstacles to

HV3C delivery

\section{Supervisors' methods}

Socratic questioning

Setting limits

Demonstrating $\mathrm{HV} 3 \mathrm{C}$ behavior

Role of workplacebased setting

\section{Supportive training program}

Interaction with supervisor

Involvement in decision-making

Practice variation

Training context

Presence of institutional policy

Availability of resources \& expertise

Clinical learning opportunities Answering questions about $\mathrm{HV} 3 \mathrm{C}$

Checking assumptions

Expressing criticism
Non-judgmental setting, open \& tolerant environment, focus on quality not on volume/costs.

$\mathrm{HV} 3 \mathrm{C}$ is a shared goal, role models are present, macro level support, critical attitude.

$\mathrm{HV} 3 \mathrm{C}$ is the heart of being a 'good' physician.

Hard to prioritize (gaining medical expertise, time management, conflict avoidance), not always desirable.

Patient determines quality and need.

Importance of $\mathrm{HV} 3 \mathrm{C}$, awareness of role as clinical teacher.

Insight into own HV3C delivery.

Reluctance to discuss $\mathrm{HV} 3 \mathrm{C}$ with patients, lack of knowledge regarding health care costs.

Open-ended questions, deducing the reasoning behind decisions.

Restrict residents' autonomy.

Communicating decisions, exploring alternatives, critical attitude toward colleagues.

Reduce anxiety (external pressure, uncertainty), reflect on necessity Invite to share ideas, determine own course of action.

Differentiate between warranted and unwarranted care, ask critical questions.

Agreements on $\mathrm{HV} 3 \mathrm{C}$ issues (cut-off values, dealing with demanding patients).

Inter- \& intra-professional collaboration, presence of supporting information, expert feedback.

What can be considered appropriate care?

Checking assumptions regarding costs of care, patient preferences and recommendations.

Mention concerns regarding appropriateness of delivered care, provide insight into line of reasoning. 


\title{
Position of this dissertation in the field of medical education
}

\author{
If doctors don't fix the problem of health care, they shouldn't be outraged \\ when outsiders try to do it for them (Jauhar, NewYorkTimes, Sept II, 20I9)
}

Medical education has the task of training medical students for the end-goal of becoming independent and competent physicians. Competent physicians meet the expectations of society. Competency frameworks commonly used for training have been developed based on these expectations. Besides the expectations of society, medical education also has the responsibility to integrate societal needs into training programs. ${ }^{24}$ This translation of societal needs into medical education is defined as social accountability (Box 1) and is not limited to HV3C delivery specifically but also applies to patient safety, quality improvement, empathy, shared decisionmaking, and patient empowerment. ${ }^{1}$

\section{Box 2:WHO definition of social accountability}

Social accountability of medical schools is the obligation to direct their education, research and service activities to addressing the priority health concerns of the community, region, and/or nation they are mandated to serve. Priority health concerns are to be identified jointly by governments, health care organizations, health professionals, and the public.

World Health Organization, Division of Development

of Human Resources for Health. Defining and Measuring

the Social Accountability of Medical Schools. Geneva:

World Health Organization 1995.'

Although it might make sense to directly link the importance of medical education to the quality and affordability of health care, their goals have not yet been properly aligned. ${ }^{25-27} \mathrm{To}$ understand the challenges that face the field of medical education, it is important to understand the history that is linked to the professional development of physicians. Historically, the medical profession was self-regulatory, based on the concept of the 'noble' physician who knew best and practiced autonomously to serve society. ${ }^{24,28}$ As a result of issues regarding harmful practice, practice variation and wasteful care, physician autonomy has been contained. Nowadays it has become a matter to be solved through a system based on accountability, measurements, and the standardization of care. This shift is often perceived by physicians as a culture of distrust, reduced autonomy, and unnecessary bureaucracy. ${ }^{28}$ Recent concerns regarding rising health care costs, the increasing power of insurance companies, and a narrow understanding of quality of care have resulted in a demand for a new shift. Claims have been made that this next shift should be a moral one, with physicians functioning completely 


\section{Pursuing High-Value, Cost-Conscious Care}

transparently, openly identifying themselves as a cog within the machine, focusing on collaboration and common goals aimed at improving health care for both the individual patient and society as a whole. Combining the pros of both eras, physicians still fulfill an important role in the new system. This would mean that harmful practices, practice variation, and wasteful care should be reduced through the self-regulatory skills of physicians. To achieve this goal, physicians should be trained as socially responsible doctors and self-regulatory health care professionals, who are conscious of their role in the general health care system. , $^{94,29,30}$ $\mathrm{HV} 3 \mathrm{C}$ is one of the topics that is essential for socially responsible doctors and deserves a more prominent place in medical training.

Residency training influences practice behavior even long after physicians have left their training environment. ${ }^{31,32}$ Postgraduate medical education is therefore an interesting starting point to train self-regulatory physicians who are able to take responsibility for the course of health care in general. Our research supports this movement by emphasizing the importance of workplacebased learning in the development of physicians' HV3C delivery and the important role residents have in seizing learning opportunities. Additionally, the societal demand for training residents to consider health care costs and to reduce wasteful practices might be supported by the research in this dissertation. Although formal education, including knowledge transmission and reflective practice, is essential, most of the physician's identity lies in the normative rules regarding behavior and responsibility that are transferred to the trainees. ${ }^{33,34}$ These normative rules start a process of moral enculturation which is the core of the hidden curriculum. Therefore, the essence of HV3C training combines formal education with a supportive environment (the hidden curriculum), sharing the values and responsibilities of socially responsible physicians. ${ }^{34}$ Besides a change in the medical profession and medical training, the macro system that should support quality of health care potentially misses its main goal of improving quality of care, since physicians aiming to deliver $\mathrm{HV} 3 \mathrm{C}$ experience a lack of support on the macro level. In general, medical education should target the hidden curriculum in all attempts to train physicians since the process of socialization is a powerful force that rarely is taken into account. Although improving the macro system is beyond the scope of this dissertation, we did indicate opportunities to support medical education and the medical profession in HV3C delivery. Areas of attention would be the financial payment structure for health services, alternative measurements of high-quality care, and a legal system that takes fear of legislation, defensive medicine and patient education into account. 


\title{
Position of this dissertation in the medical profession
}

\author{
"So, what is value, and who decides?" Lisa Rosenbaum, March 7, 2013.
}

A central theme in this dissertation is value within the health care system. The concept of value depends heavily on who is asked to define it. The shift toward patient-centered care, characterized by increased patient involvement in decision-making and growing emphasis on the importance of patient autonomy, ${ }^{35}$ raised awareness of the patient's perspective on value. Literature is divided regarding the relation between patient preferences and highvalue, cost-conscious care. Shared decision making (SDM) literature claims that correct administration of SDM-principles for treatment options increases the delivery of high-value care. Treatment options in such cases are based on the latest scientific evidence and are selected based on their appropriateness. ${ }^{36}$ However, not all medical decision-making has such demarcated set of suitable testing, imaging or treatment for a symptom or disease. Furthermore, it is known that patients value imaging techniques, even when evidence-based medicine identifies imaging as unnecessary. ${ }^{37,38}$ Additionally, when patients visit the emergency department, doing a CT scan increases patient satisfaction more than a physical examination. ${ }^{39}$ With this knowledge, it becomes apparent that centering a health care system on high-value care from the patient's perspective could fasten or hamper HV3Cdelivery. If patients request options that are not suitable for their symptom or disease this could create tension between the physicians' obligations. Physicians are obliged to correctly inform, guide, and support the patient during their journey through the health care system ${ }^{40,41}$ while preventing patients from harm, respecting their personal preferences and safeguarding the sustainability of health care. Unfortunately, 'do no harm' and patient preferences do not always go hand in hand, and even if they do, that does not necessarily result in sustainable health care. Physicians may find these conflicting obligations, formally described in professional competence frameworks, ${ }^{42,43}$ the Hippocratic oath ${ }^{41}$, and in medical codes ${ }^{40,44}$ difficult and perhaps impossible to balance. It raises the question of who should have the final say when it comes to care delivery and the patient's desire for certain diagnostic services or treatment. Some would say that patients can decide for themselves what can be considered valuable health care, after being correctly informed about the pros and cons, ${ }^{40,44}$ even if these decisions are of questionable value in terms of the medical profession, or harmful to the sustainability of health care. If patient preferences lead care delivery, there might be a group of patients who prefer dubious care and who are not open to their physician's advice or are not susceptible to scientific evidence. ${ }^{26}$

"Once a patient has decided that something is necessary, challenging that perception with evidence is no easy feat." (Lisa Rosenbaum ${ }^{26}$ ) 


\section{Pursuing High-Value, Cost-Conscious Care}

Others would plead that it is the physician's duty to prevent unnecessary antibiotic or pain medication prescriptions and that the physician 'knows best' in some situations. But how can physicians determine if they are allowed to 'know best' and if they are permitted to refuse treatment to their patients, even after patients have received extensive information about the potential harm and are deemed capable of making a decision? And what if reassured patients, even as a result of unnecessary additional tests, feel happier, less stressed and confident about their personal health? Can we still claim that these tests are unnecessary? This is especially difficult when the scientific claims regarding diagnostic/treatment options seem to be continuously discussed ${ }^{26}$ without clear recommendations. Therefore, it is not surprising that some physicians are willing to concede to patients wishes, not in the least as a result of lawsuits and complex discussions. The medical profession should participate in the discussion who determines high-value care. The challenge lies in identifying under which circumstances it is possible to meet patient expectations without delivering (too much) wasteful or harmful care, and letting patients determine what can be considered 'high-value care' themselves. Physicians should reflect on their personal interests, motives, and how these interests influence their medical decision-making. Exploring where patient preferences and physicians' responsibilities can coexist is impossible to dictate and should be assessed in the context of a specific case. In HV3C delivery, deliberating on such topics with peers, trainees and patients could be helpful in determining where conflicts of interest occur.

\section{Practical implications}

The practical implications of this dissertation are discussed in Chapter 6 (tips for training residents in $\mathrm{HV} 3 \mathrm{C}$ delivery). An overview of these tips is presented below in Table 9.

Table 9: I 2 tips for training high-value, cost-conscious care in the clinical setting

Tip I Reflect on the personal stance of residents and supervisors.

Tip 2 Ask and answer critical questions regarding future and past care delivery.

Tip 3 Be aware of the important role of supervisors in residency training.

Tip $4 \quad$ Provide opportunities to pursue proposed diagnostic and treatment plans.

Tip 5 Engage medical students and residents in discussions regarding complex cases.

Tip 6 Stimulate residents to seek external expertise and build interprofessional collaborations.

Tip 7 Apply utilization data to reflect on personal and collective care delivery.

Tip $8 \quad$ Use practice variation as a starting point for discussing what care is deemed appropriate.

Tip 9 Encourage residents to explicate patient preferences and expectations of the health care system.

Tip $10 \quad$ Talk about health care costs with patients.

Tip II Discuss within the department how to deal with demanding patients and guideline adherence.

Tip 12 Seek to expand teaching methods to training during supervision. 


\section{Limitations}

We used various methods to explore how medical students, residents and physicians learn to deliver high-value, cost-conscious care. This dissertation combines several qualitative methods to answer the overarching research question. Our results are thus built on knowledge of effective educational interventions and theory and descriptions of how residents are trained in HV3C delivery.

That this qualitative study is limited to the Dutch setting may potentially influence our results. Health care systems, insurance systems, legal systems, professional identities and social expectations are known to vary between countries and therefore national results will not always be transferable to foreign settings. Nevertheless, since many countries train residents in more or less similar manners, our findings might resonate with clinical educators, residents, medical students and educationalist outside the Netherlands. Although this dissertation aims to provide insight into how $\mathrm{HV} 3 \mathrm{C}$ is trained in the postgraduate setting, due to the sheer number of training programs, not all the specialties were included in the studies performed. To address this limitation, we purposefully sampled participants from a range of training programs and aimed to identify how training is generally perceived and approached so that we had an overview of surgical, nonsurgical, hospital and non-hospital-based specialties. Among the participating training programs, our data demonstrated differences that might give specific information on different needs per residency program and warrants further research. Yet, since our aim was to gain a general understanding, we did not aim to further unravel how needs differed between programs, although this is relevant to providing tailored support to residency programs. To explore different needs per training program and learn from 'best practices', further research is necessary.

A recurrent limitation in this dissertation is the different conceptions of $\mathrm{HV} 3 \mathrm{C}$ and the presence of social desirability related to the increased attention for HV3C. By sharing a formal definition with all participants, we aimed to prevent confusion regarding the topic of our research. Due to the increasing societal awareness for $\mathrm{HV} 3 \mathrm{C}$ delivery and training, participants might have valued HV3C more positively. We aimed to limit this influence by asking participants to underpin their attitudes and experiences with examples from daily practice. In our ethnographic study, we put much effort into getting acquainted with the participants and stretching the time our study took place in the department. In doing so, we aimed to gain access to daily routines and approaches to HV3C delivery and limit the influence of social desirability.

Data analysis is known to be influenced by the background of the researchers, which is why we included a reflexivity section in the general introduction. Doing research on residency training while being admitted to a residency training program colored our data analysis and can be 
considered both a strength and a limitation of this $\mathrm{PhD}$ project. The presence of a stable team of experienced researchers and clinicians, combined with several co-authors for all studies, led to fruitful discussions about the data analysis process.

Another limitation worth mentioning is the subjectivity linked to HV3C. There is no objective cut-off point for HV3C and the appropriateness of health care services is debatable. In order to make claims regarding the appropriateness of services, knowledge of the specific case, context, examination and topic-specific medical knowledge must be present. Due to the absence of these factors, we did not judge whether the delivered care in this study meets the definition of HV3C but focused for example on how discussions regarding appropriateness were part of residency training or how residents were stimulated consider HV3C delivery.

\section{Suggestions for further research}

This dissertation was conducted with the aim of exploring how residents are trained in HVC3 delivery. Future research is necessary to assess the current level of HV3C-delivery and should look further than attitudes and self-reported behavior of (future) physicians. Also, since medical students are the residents of the future, it is interesting to gain understanding of when medical education should introduce $\mathrm{HV} 3 \mathrm{C}$ in the medical curriculum and how undergraduate and postgraduate training could complement each other. However, it is important to bear in mind that the level of complexity of determining what can be considered HV3C might need to be reduced in order to avoid confusing novice medical students. Future research could focus on the timing, level of complexity and alignment with the postgraduate program.

This dissertation is only a first small step in unraveling how physicians learn to deliver high-value, cost-conscious care. For the field of medical education, it remains important to further explore the influence of the environment in HV3C training. Although our research focused on HV3C delivery, it might be valuable to combine it with other themes (for example: patient safety, empathy, shared decision-making) since these themes are also strongly influenced by the environment in which physicians are trained. It could be wise to develop an educational intervention to improve training in the workplace-based setting. As we concluded in Chapter 5, an external expert might be useful to pose critical questions and identify any knowledge deficits present in the department. In such a design, training should be tailored to the needs of both staff members and residents and should focus on making HV3C a topic that is propagated by the entire department. 
Table 10: Areas of future research

I. Research on HV3C delivery in practice, beyond self-reported behavior and attitudes.

2. Focus on the undergraduate training program and its alignment to the postgraduate program.

3. Combine research areas in which the support of the environment is important for training.

4. Develop and assess educational intervention aiming to improve $\mathrm{HV} 3 \mathrm{C}$ training and faculty teaching

5. Include health care professionals in future research.

6. Explore differences in needs between residency training programs.

7. Focus on training adaptive physicians that can function as change agents.

In addition, in order to train faculty for their role in HV3C delivery it is important to acknowledge the socialization processes that are fundamental to 'becoming faculty'. ${ }^{45}$ Since our research indicated that interprofessional collaboration with health care professionals is important, it might be necessary to focus more on the role of other health care professionals (for example nurses, physiotherapist, social workers etc.) in the training of residents and how they approach HV3C during their own clinical tasks.

In the limitation section, we discussed that our research data suggests differences between training programs. In order to provide tailored support to training programs, it might be worth considering differences between training programs and how these can be translated into HV3C training. Ultimately, future research should focus on the development of independent, critical health care professionals, who are able to adjust their training and care delivery on societal needs. Besides life-long learning to maintain high levels of skills and knowledge, physicians should be prepared to lead the profession in the ever-changing health care system. In order to create such leaders, it is important to focus on 'how' professionals learn and 'from whom' they learn, rather than focusing on 'what' is learned and 'when' it is learned. Again, it is likely that workplace-based culture is an important factor that deserves a key role in future research.

\section{References}

1. World Health Organisation DoD, Health. oHRf. Defining and Measuring the Social Accountability of Medical Schools. Geneva1995.

2. Nguyen QD, Fernandez N, Karsenti T, Charlin B. What is reflection? A conceptual analysis of major definitions and a proposal of a five-component model. Med Educ. 2014;48(12):1176-1189.

3. Fragkos K. Reflective practice in healthcare education: an umbrella review. Education Sciences. 2016;6(3):27.

4. von Ferber L, Bausch J, Koster I, Schubert I, Ihle P. Pharmacotherapeutic circles. Results of an 18-month peer-review prescribing-improvement programme for general practitioners.

Pharmacoeconomics. 1999;16(3):273-283. 


\section{Pursuing High-Value, Cost-Conscious Care}

5. King BC, DiPace J, Naifeh M, Hammad H, Gerber LM, Abramson E. Pediatric Training Faculty and Resident Perceptions on Teaching High-Value, Cost-Conscious Care: A Multi-Institutional Study. Hosp Pediatr. 2017;7(9):547-552.

6. Cruess RL, Cruess SR, Boudreau JD, Snell L, Steinert Y. A schematic representation of the professional identity formation and socialization of medical students and residents: a guide for medical educators. Acad Med. 2015;90(6):718-725.

7. Danis M, Sommers R, Logan J, et al. Exploring public attitudes towards approaches to discussing costs in the clinical encounter. J Gen Intern Med. 2014;29(1):223-229.

8. Jonas JA, Ronan JC, Petrie I, Fieldston ES. Description and Evaluation of an Educational Intervention on Health Care Costs and Value. Hosp Pediatr. 2016;6(2):72-79.

9. Gupta R, Steers N, Moriates C, Wali S, Braddock CH, 3rd, Ong M. High-Value Care Culture Among the Future Physician Workforce in Internal Medicine. Acad Med. 2019;94(9):1347-1354.

10. Legare F, Labrecque M, LeBlanc A, et al. Training family physicians in shared decision making for the use of antibiotics for acute respiratory infections: a pilot clustered randomized controlled trial. Health Expect. 2011;14 Suppl 1:96-110.

11. Gist D, Llorente J, Mayer J. A clinical algorithm for the management of abnormal mammograms. A community hospital's experience. West J Med. 1997;166(1):21-28.

12. Lyle CB, Jr., Bianchi RF, Harris JH, Wood ZL. Teaching cost containment to house officers at Charlotte Memorial Hospital. J Med Educ. 1979;54(11):856-862.

13. Zeleznik C, Gonnella JS. Jefferson Medical College Student Model Utilization Review Committee.J Med Educ. 1979;54(11):848-851.

14. Sucov A, Bazarian JJ, deLahunta EA, Spillane L. Test ordering guidelines can alter ordering patterns in an academic emergency department. J Emerg Med. 1999;17(3):391-397.

15. McKay RM, Vrbova L, Fuertes E, et al. Evaluation of the Do Bugs Need Drugs? program in British Columbia: Can we curb antibiotic prescribing? Can J Infect Dis Med Microbiol. 2011;22(1):19-24.

16. Cammisa C, Partridge G, Ardans C, Buehrer K, Chapman B, Beckman H. Engaging physicians in change: results of a safety net quality improvement program to reduce overuse. Am JMed Qual. 2011;26(1):26-33.

17. Colbert CY, Ogden PE, Lowe D, Moffitt MJ. Students learn systems-based care and facilitate system change as stakeholders in a free clinic experience. Adv Health Sci Educ Theory Pract. 2010;15(4):533-545.

18. Spiegel CT, Kemp BA, Newman MA, Birnbaum PS, Alter CL. Modification of decision-making behavior of third-year medical students. J Med Educ. 1982;57(10 Pt 1):769-777.

19. Post J, Reed D, Halvorsen AJ, Huddleston J, McDonald F. Teaching high-value, cost-conscious care: improving residents' knowledge and attitudes. Am JMed. 2013;126(9):838-842.

20. Polinski JM, Schneeweiss S, Maclure M, Marshall B, Ramsden S, Dormuth C. Time series evaluation of an intervention to increase statin tablet splitting by general practitioners. Clin Ther. 2011;33(2):235-243.

21. Niedermier V. Gap in Residency Education in Dealing With Uncertainty. Fam Med. 2016;48(5):403-404.

22. Rohacek M, Albrecht M, Kleim B, Zimmermann H, Exadaktylos A. Reasons for ordering computed tomography scans of the head in patients with minor brain injury. Injury. 2012;43(9):1415-1418.

23. Perez R, Aizenberg D, Davis T, Ryskina KL. Seeking a stable foundation to build on: 1(st)-Year residents' views of high-value care teaching. Educ Health (Abingdon). 2019;32(1):11-17.

24. Woollard RF. Caring for a common future: medical schools' social accountability. Med Educ. 2006;40(4):301-313. 
25. Gupta R, Arora VM. Merging the Health System and Education Silos to Better Educate Future Physicians. JAMA. 2015;314(22):2349-2350.

26. Rosenbaum L. The whole ball game--overcoming the blind spots in health care reform. NEnglJ Med. 2013;368(10):959-962.

27. Sklar DP, Hemmer PA, Durning SJ. Medical Education and Health Care Delivery: A Call to Better Align Goals and Purposes. Acad Med. 2018;93(3):384-390.

28. Berwick DM. Era 3 for Medicine and Health Care. JAMA. 2016;315(13):1329-1330.

29. Cooke M. Cost consciousness in patient care--what is medical education's responsibility? N Engl J Med. 2010;362(14):1253-1255.

30. Pellegrino ED. Academic health centers and society: an ethical reflection. Academic medicine: journal of the Association of American Medical Colleges. 1999;74(8 Suppl):S21-26.

31. Asch DA, Nicholson S, Srinivas S, Herrin J, Epstein AJ. Evaluating obstetrical residency programs using patient outcomes. JAMA. 2009;302(12):1277-1283.

32. Bressers G, Wallenburg I, Stalmeijer R, Oude Egbrink M, Lombarts K. Patient safety in medical residency training: Balancing bravery and checklists. Health (London). 2020:1363459319899444.

33. Hafferty FW, Franks R. The hidden curriculum, ethics teaching, and the structure of medical education. Acad Med. 1994;69(11):861-871.

34. Gupta R, Moriates C. Swimming Upstream: Creating a Culture of High-Value Care. Acad Med. 2017;92(5):598-601.

35. Mead N, Bower P. Patient-centredness: a conceptual framework and review of the empirical literature. Soc Sci Med. 2000;51(7):1087-1110.

36. King TL, Likis FE, Wilson-Liverman AM. Shared Decision Making: Misconstrued and Misused.J Midwifery Womens Health. 2018;63(3):257-258.

37. Kendrick D, Fielding K, Bentley E, Kerslake R, Miller P, Pringle M. Radiography of the lumbar spine in primary care patients with low back pain: randomised controlled trial. $B M J$. 2001;322(7283):400-405.

38. Ash LM, Modic MT, Obuchowski NA, Ross JS, Brant-Zawadzki MN, Grooff PN. Effects of diagnostic information, per se, on patient outcomes in acute radiculopathy and low back pain. AJNR Am J Neuroradiol. 2008;29(6):1098-1103.

39. Baumann BM, Chen EH, Mills AM, et al. Patient perceptions of computed tomographic imaging and their understanding of radiation risk and exposure. Ann Emerg Med. 2011;58(1):1-7 e2.

40. Council GM. The duties of a doctor registered with the General Medical Council. Published 2013. Accessed 25-02-2020, 2020.

41. Geneeskunst KNM Nederlandse artseneed. www.knmg.nl2009. Accessed 13-02, 2020.

42. Holmboe E. ACGME milestones framework. https://www.acgme.org. Published 2015. Accessed2019.

43. Frank JR, Snell L, Sherbino J, eds. CanMEDS 2015 Physician Competency Framework. Ottawa, Ontario, Canada: Royal College of Physicians and Surgeons of Canada; 2015.

44. Association TWM. World Medical Association Medical Ethics Manual. 2015.

45. Hafler JP, Ownby AR, Thompson BM, et al. Decoding the learning environment of medical education: a hidden curriculum perspective for faculty development. Acad Med. 2011;86(4):440-444. 
Summary 
Increasing costs of health care are a cause of concern to patients, governments, health economists, and the medical profession worldwide. Chapter I describes how these rising health care expenditures jeopardize the sustainability of health care systems that ensure acceptable, accessible, equitable, and affordable delivery of high-quality care. This dissertation explores how medical education can contribute to the delivery of high-quality care while simultaneously acknowledging rising health care costs. Medical education attempts to prepare physicians for their role in sustainable health care, as providers of high-value, cost-conscious care (HV3C). HV3C is care that aims to assess the benefits, harms, and costs of interventions and consequently to provide care that adds value.

This dissertation investigates the role of medical education in the training of high-value, costconscious care delivery using the following research questions:

\section{Which elements of educational interventions are effective to train (future) physicians in the delivery of high-value, cost-conscious care?}

2 How do residents approach the delivery of high-value, cost-conscious care in daily practice?

3 How do supervisors aim to train residents in the delivery of high-value, cost-conscious care?

4 How is the workplace setting related to residents' high-value, cost-conscious care delivery?

Chapter 2 aims to gain understanding of how and under what circumstances educational interventions may help practicing physicians, resident physicians, and medical students deliver high-value, cost-conscious care. Insight into this learning process is necessary to develop programs that train physicians in providing such care. Our realist review indicates that effective educational interventions rely on a combination of three elements: knowledge transmission, reflective practice and a supportive environment. Knowledge transmission entails knowledge of the general economics of health care and its costs, knowledge of patient preferences and scientific knowledge (medical expertise). Reflective practice aims to help physicians gain insight into their performance with the goal of influencing future behavior. Providing feedback, stimulating reflection, or a combination of both are used to prepare trainees for HV3C delivery. Finally, the presence of a supportive environment is characterized by the presence of a communal goal to deliver HV3C shared by clinical role models and teachers alike, and a culture of interprofessional collaboration in relation to high-value, costconscious care. Our review concluded that besides support from 'working staff, support on the macro level, including the organizational structure is crucial.

Chapter 3 focuses on residents' perception of HV3C training in the workplace-based setting. Residents from six different residency training programs participated in focus groups that discussed their training methods and how the learning environment can support workplacebased learning. Influential factors in learning HV3C delivery operate on three levels: the 


\section{Pursuing High-Value, Cost-Conscious Care}

individual resident, the training program, and the workplace level. On the individual level the spectrum ranges from 'the frugal type' who is always concerned with HV3C to the 'costs-are-noton-my-mind' type, describing the resident who cannot be bothered by financial concerns. In between, we identified a group with a more moderate attitude that can be described as mindful of $\mathrm{HV} 3 \mathrm{C}$ but seems to find it difficult to prioritize it along with other tasks in daily practice. These residents are illustrated by this participant's quote: "It's our responsibility to deliver HV3C but..." At the training program level, perceived determinants of learning include residentsupervisor interactions, involvement in decision-making over time, and exposure to variation in care delivery. At the workplace level, learning depends on the availability of professional health care expertise and on the presence of institutional policy.

Chapter 4 deals with the key figures of supervisors, exploring how they aim to prepare residents for $\mathrm{HV} 3 \mathrm{C}$ delivery. How they do this is based on the priority given to HV3C training, the presence of feedback on HV3C, and the supervisors' perceived obstacles to HV3C delivery. Reluctance to discuss health care costs and a lack of knowledge of health care costs were often mentioned as barriers in training residents. Results further indicate that attending physicians use three methods to teach HV3C delivery: Socratic questioning, role modeling, and setting limits. Applying these methods supervisors, often implicitly, aim to train residents to deliver HV3C in the workplace setting.

Chapter 5 aims to unravel how the learning environment relates to the HV3C delivery by residents. We observed gynecology residents in daily practice delivering clinical care interviewed both residents and supervisors of the participating department. Our study identified how residents consider the risks, costs and benefits of health care services in practice and how the attending physician influences this process. We noticed that financial costs are rarely discussed in determining what care could be considered as 'appropriate'. Additionally, we noted some variation between supervisors that was confusing for junior residents. We concluded that three main areas for learning could be improved: questions about HV3C should be answered, and the answers to those questions should be checked in order to avoid decision-making based on (false) assumptions. Disagreement within the department should lead to open, face-to-face discussion. If criticism is shared, it should become clear why physicians chose different approaches in a specific patient case. Interview data suggests that residents feel the culture does not fully support sharing criticism, while supervisors feel that these discussions would confuse residents on what could (or should) be considered appropriate care.

Chapter 6 aims to translate our scientific findings into practical tips in support of HV3C training for residents. The tips target clinical educators for training in daily practice but can be translated into the resident perspective. 
Table 9: 12 tips for training high-value, cost-conscious care in the clinical setting

Tip I Reflect on the personal stance of residents and supervisors.

Tip 2 Ask and answer critical questions regarding future and past care delivery.

Tip 3 Be aware of the important role of supervisors in residency training.

Tip 4 Provide opportunities to pursue proposed diagnostic and treatment plans.

Tip 5 Engage medical students and residents in discussions regarding complex cases.

Tip 6 Stimulate residents to seek external expertise and build interprofessional collaborations.

Tip 7 Use utilization data to reflect on personal and collective care delivery.

Tip 8 Use practice variation as a starting point for discussing which care is deemed appropriate.

Tip 9 Encourage residents to explicate patient preferences and expectations of the health care system.

Tip 10 Talk about health care costs with patients.

Tip II Discuss in the department how to deal with demanding patients and guideline adherence.

Tip 12 Seek to expand teaching methods to train during supervision.

Chapter $\mathbf{7}$ deals with the coherence between chapters and puts the findings into a broader perspective. We discuss our research in the light of medical education's responsibility for translating societal needs into training programs. This responsibility is not specific to HV3C but extends to other domains and topics, such as patient safety and elderly care. Furthermore, we discuss HV3C in the light of the profession and conflicts of interests that occur when physicians should protect the sustainability of health care but are obligated to put the patient first. Physicians are challenged by differing expectations which, we would like to point out, could create barriers for the delivery of HV3C. These barriers influence attempts to train residents in their role in providing $\mathrm{HV} 3 \mathrm{C}$ and hinder the development of a supportive environment for HV3C delivery. 
Samenvatting 
Stijgende zorgkosten zijn reden tot zorg voor patiënten, overheden, gezondheidseconomen en de medische professie wereldwijd. Hoofdstuk I beschrijft hoe de stijgende zorguitgaves de toegankelijkheid, betaalbaarheid, en gelijkwaardigheid van het gezondheidssysteem bedreigen. In dit proefschrift verkennen we hoe medisch onderwijs kan bijdragen aan het leveren van hoogwaardige zorg door artsen waarbij de stijgende zorgkosten gelijktijdig in ogenschouw genomen worden. Medisch onderwijs tracht artsen voor te bereiden op hun werk binnen een duurzaam zorgsysteem dat doelmatige zorgt levert. Doelmatige zorg beoogt de voordelen, nadelen en kosten van medische interventies te balanceren en zodoende zinnige zorg te leveren.

\section{Dit proefschrift onderzoekt de rol van medisch onderwijs in het opleiden van artsen voor} het leveren van doelmatige zorg door middel van de volgende onderzoeksvragen:

2 Welke aspecten van onderwijskundige interventies zijn effectief binnen het opleiden van (toekomstige) artsen in het leveren van doelmatige zorg?

3 Hoe gaan artsen-in-opleiding-tot-specialist (AIOS) om met het leveren van doelmatige zorg?

4 Hoe bereiden supervisoren AIOS voor op het leveren van doelmatige zorg?

5 Welke rol speelt het werkplek-leren bij het leveren van doelmatige zorg door AIOS?

In hoofdstuk 2 onderzochten we hoe, onder welke omstandigheden en waarom onderwijskundige interventies artsen, AIOS, en medisch studenten ondersteunen in het leveren van doelmatige zorg. Inzicht in dit leerproces is essentieel voor het ontwikkelen van effectieve onderwijsprogramma's gericht op het leren leveren van doelmatige zorg. Onze realist review laat zien dat effectieve onderwijskundige interventies zich richten op het combineren van drie elementen: kennisoverdracht, reflectie op eigen handelen en een ondersteunende omgeving. Kennisoverdracht richt zich op het verwerven van kennis over algemene gezondheids-economische principes en kosten van de zorg, kennis van patiënt-voorkeuren en wetenschappelijk bewijs voor bijvoorbeeld behandelingen en diagnostiek. Reflectie op eigen handelen is noodzakelijk voor het verkrijgen van inzicht in de geleverde zorg met als doel leerpunten te identificeren voor toekomstige gedrag. Het geven van feedback op en het stimuleren van reflectie op het handelen van de arts (of een combinatie van beiden) worden gebruikt om reflectie op eigen handelen te stimuleren. Daarnaast is de aanwezigheid van een ondersteunende omgeving belangrijk, welke zich kenmerkt door een algemeen gedragen belang van het leveren van doelmatige zorg. Verder kenmerkt deze omgeving zich door de aanwezigheid van rolmodellen en docenten voor doelmatige zorg, en waarbij interprofessionele samenwerking rondom doelmatigheid hoog in het vaandel staat. Ondersteuning op macroniveau, zoals bijvoorbeeld het bestuur van een ziekenhuis of het managementteam van een zorginstelling, is tevens cruciaal.

Hoofdstuk 3 richt zich op de percepties van AIOS over het opleiden in doelmatige zorg op de werkplek. Arts-assistenten van zes verschillende opleidingsprogramma’s namen deel in homogene focusgroepen om te bespreken hoe hun opleiding aandacht besteedt aan doelmatigheid, en hoe de 


\section{Pursuing High-Value, Cost-Conscious Care}

werkplek het opleiden in doelmatige zorg kan ondersteunen. Het leren leveren van doelmatigheid wordt beïnvloed op drie niveaus: de AIOS als individu, de opleiding, en de werkplek. Op het niveau van het individu beschrijven we een spectrum aan typeringen rondom de houding van de AIOS ten opzichte van doelmatigheid variërend van 'het zuinige type': iemand die altijd en overal bezig is met het zuinig en zinnig leveren van zorg tot het 'ik hou me niet bezig met kosten-type', welke de AIOS beschrijft die zich niet verantwoordelijk voelt voor de kosten van de gezondheidszorg. Daartussen zit een grote groep van AIOS die zich bewust zijn van hun verantwoordelijkheid ten aanzien van doelmatige zorg, maar moeite hebben er prioriteit aan te geven in de dagelijkse praktijk. Deze AIOS worden beschreven door de karakteristieke quote "het is onze verantwoordelijkheid om doelmatige zorg te leveren, maar....". Op het niveau van de opleiding spelen de interactie tussen AIOS en supervisor, de betrokkenheid van AIOS bij het nemen van beslissingen en de blootstelling aan variatie en hoe en welke zorg geleverd wordt een belangrijke rol. Op de werkplek hangt het leren van doelmatige zorg sterk samen met de beschikbaarheid van medische expertise en de aanwezigheid van een afdelings- en ziekenhuisvisie rondom doelmatigheid.

In Hoofstuk 4 staat de rol van de supervisor centraal en wordt onderzocht hoe supervisoren AIOS voorbereiden op het leveren van doelmatige zorg. Gedurende semigestructureerde interviews deelden supervisoren dat de wijze waarop zij AIOS voorbereiden sterk samenhangt met de prioriteit die gegeven wordt aan doelmatige zorg, de anwezigheid van feedback en de barrières die door de supervisoren ervaren worden ten aanzien van het leveren van doelmatige zorg. Het nalaten van het bespreken van zorgkosten en een gebrek aan kennis ten aanzien van zorgkosten wordt vaak genoemd door supervisoren als een barrière. Onze resultaten laten verder zien dat supervisoren gebruik maken van drie didactische principes om doelmatige zorg te integreren in de opleiding. Deze principes zijn het socratisch bevragen van de AIOS, het zijn van een rolmodel rondom doelmatigheid en het stellen van grenzen met betrekking tot autonome beslissingen voor diagnostiek of behandeling. Door het gebruiken van deze principes proberen supervisoren, vaak impliciet, AIOS voor te bereiden op het zelfstandig leveren van doelmatige zorg.

Hoofdstuk 5 gaat in op de relatie tussen de leeromgeving en het leveren van doelmatige zorg door AIOS. Door middel van observationeel onderzoek hebben we inzicht gekregen in de dagelijkse bezigheden van AIOS op de werkplek en de rol die doelmatigheid hierbij speelde. Als aanvulling op onze observaties hebben we zowel AIOS als supervisoren geïnterviewd, om nader in te gaan op onze bevindingen uit de observaties. De resultaten uit deze studie laten zien hoe AIOS afwegingen maken tussen de voordelen, nadelen en kosten van de zorg en hoe de supervisoren deze afweging beïnvloeden. Uit onze observaties bleek dat er weinig aandacht was voor de bespreking van kosten in gesprekken waarin dit wel gepast zou zijn geweest. Verder werd duidelijk dat variatie in handelen tussen supervisoren verwarrend kon zijn voor jonge AIOS. Om het leren op de werkplek te ondersteunen zou er meer aandacht mogen zijn voor vragen rondom doelmatige zorg en bijbehorende antwoorden. Deze antwoorden zouden getoetst moeten worden om te voorkomen 
dat de beslisvorming gebaseerd wordt op onjuiste informatie. Daarnaast kunnen meningsverschillen rondom doelmatigheid kansen bieden voor open discussies binnen het zorgteam. De achtergronden van deze meningsverschillen zou gedeeld moeten worden, zodat het voor AIOS duidelijk is waar verschillende standpunten hun oorsprong vinden. De bevindingen van de interviews geven aan dat AIOS een cultuur ervaren waarin elkaar kritisch benaderen niet altijd wenselijk is, terwijl supervisoren het idee hebben dat dergelijke discussies verwarrend zouden zijn voor AIOS.

Om opleiders, supervisoren en AIOS te ondersteunen bij het opleiden in het leveren van doelmatige zorg zijn een aantal onderzoeksbevindingen vertaald in praktische tips in Hoofdstuk 6. De tips zijn geschreven vanuit het perspectief van de supervisor, maar kunnen eenvoudig vertaald worden naar het perspectief van de AIOS.

\section{2 tips voor het opleiding in doelmatige zorg}

$\begin{array}{ll}\text { tip I } & \text { Ben u bewust van uw eigen ten aanzien van doelmatige zorg } \\ \text { tip 2 } & \text { Stel kritische vragen over doelmatigheid } \\ \text { tip 3 } & \text { Wees u bewust van uw functie als rolmodel voor het leveren van doelmatige zorg } \\ \text { tip } 4 & \text { GeefAIOS de ruimte voor het vervolgen van eigen beleidsvoorstellen } \\ \text { tip 5 } & \text { BetrekAIOS in discussies rondom complexe casuïstiek } \\ \text { tip 6 } & \text { Stimuleer AIOS om advies in te winnen buiten de vakgroep } \\ \text { tip 7 } & \text { Vraag/zoek spiegel-informatie en gebruik deze voor reflectie } \\ \text { tip 8 } & \text { Maak variatie tussen collega's bespreekbaar } \\ \text { tip 9 } & \text { Bespreek altijd expliciet de hulpvraag van de patiënt } \\ \text { tip I0 } & \text { Let's talk about money. } \\ \text { tip II } & \text { Neem als vakgroep een collectief standpunt in } \\ \text { tip I2 } & \text { Maak gebruik van onderwijskundige ondersteuning }\end{array}$

Hoofdstuk 7 verbindt de bevindingen van ons onderzoek met de grotere maatschappelijke en medisch onderwijskundige discussie. We bespreken de verantwoordelijkheid van medisch onderwijs om maatschappelijke behoeftes te integreren in de medische vervolgopleiding. Deze verantwoordelijkheid is niet beperkt tot het thema doelmatigheid, maar geldt net zozeer voor andere onderwerpen zoals patiëntveiligheid, kwaliteitsverbetering en ouderengeneeskunde. Er is een spanningsveld voor artsen rondom het behartigen van de belangen van de individuele patiënt en tegelijkertijd het borgen van de beschikbaarheid en betaalbaarheid van de zorg. Deze verschillende belangen en verwachtingen kunnen het lastig maken voor artsen om doelmatige zorg te leveren. Dit spanningsveld beïnvloedt het opleiden van AIOS in hun taak om doelmatige zorg te leveren maar hindert ook het creëren van een ondersteunende omgeving om die zorg te leveren. 
Valorization 
This valorization chapter discusses the importance of the research within this doctoral thesis for the general public. With the exponential growth of health care costs worldwide, the practice of high-value, cost-conscious care is essential for a sustainable future of health care. This dissertation describes the essential role of training throughout the medical education continuum in teaching student, residents and physicians to deliver high-value, cost-conscious care. As such, lessons learnt from this thesis can be applied to medical education, patients and society. First, we will discuss the benefits for post-graduate medical training, followed by the effect on patients and society.

\section{How this thesis contributes to the training of physicians in the delivery of high-value, cost-conscious care.}

The results of this thesis give insight in how postgraduate training prepares residents for the delivery of high-value, cost-conscious care. We identified the importance of three elements of educational interventions: knowledge-transmission, reflective practice and the presence of a supportive environment. This supportive environment consists of role models and health care professionals that value and demonstrate high-value, cost-conscious care. Our results give direction for the development of future educational interventions. Since residents spend the majority of their training in the clinical workplace environment, we aimed to gain more insight in how this workplace-based learning is organized. A few important conclusions are worth summarizing in this chapter. First, the individual resident approach determines if residents seek and seize learning opportunities. Residents vary greatly in how they approach HV3C in their workplace setting and knowledge regarding differences in approaches can facilitate training. For those involved in teaching it is crucial to be aware of these individual approaches and how they influence individual learning. Secondly, the supervisor is identified as a key figure in this learning process. Supervisors' individual approach towards HV3C influence the implicit and explicit training of residents. Certain barriers, for example the lack of knowledge of health care costs or the absence of feedback data, are present and hinder the learning process of residents. To support the training of residents, we would advise to closely guide and support supervisors in their role as clinical teachers. It could be a task of the training program to set conditions that facilitate training in general. For example, by supporting interprofessional collaboration and encourage involvement in decision-making and long-term patient contact. Our research identified some barriers in the workplace setting and residents' benefit from overcoming those barriers. The most important barriers are the unfinished discussions including questions about high-value costconscious care that are not answered or incorrect information that is shared among professionals. In addition, we observed that face-to-face discussions regarding delivered care are scarce although they potentially create valuable learning opportunities for residents. The final chapter of this dissertation is an overview of practical tips for both supervisors, program directors and residents 


\section{Pursuing High-Value, Cost-Conscious Care}

to improve training in the workplace setting. By using these tips, medical education in general can transform towards a training environment that is able to train formally and informally in the delivery of HV3C.

Our research contributes to the importance of $\mathrm{HV} 3 \mathrm{C}$ in postgraduate medical education and the current debate on how residents should be trained herein. At several international and national conferences, we joined the debate on HV3C-delivery and its training. By presenting at both medical, educational and topic-specific conferences we aimed to increase the impact of our research and gain insight in differences between communities. Our research contributed to these debates with scientific evidence on current training and suggestions for the improvement of training. We aimed to publish in both educational and medical journals to reach an audience that researches, develops and trains future physicians, to create impact at the core of postgraduate education. In addition, our results provide guidance to further investigate how physicians can be trained in the delivery of HV3C in both undergraduate and postgraduate settings, in different countries and different medical specialties. Therefore, this dissertation is merely the beginning of unravelling the complex world of $\mathrm{HV} 3 \mathrm{C}$ with the potential to influence training and care delivery at grass root level.

\section{How the delivery of HV $3 \mathrm{C}$ by physicians contributes to the quality and affordability of our health care system.}

When we are able to improve the postgraduate training of physicians in delivering high-value, cost-conscious care we contribute to sustainable healthcare for all people who use the health care system. A sustainable health care system is important since it influences the general wellbeing of the population by impacting the economic, mental and physical wellbeing. Also, by focusing on the delivery of $\mathrm{HV} 3 \mathrm{C}$ the population is less likely to experience the adverse effects of overdiagnosis, overtreatment and anxiety that comes with incidental findings. HV3C aims to be patientcentered, by openly discussing the patient's preferences and expectations. By clearly stating these preferences and expectations the physician and patient can share in the decision-making what care would be considered appropriate. The delivery of HV3C would increase the quality of health care without or containing increasing health care costs. Rising health care costs are a big concern of politicians, economists and citizens in de western world and there are many interventions implemented to bend this rise. Unfortunately, many interventions do not combine health care quality and health care costs, potentially disturbing the essential balance between both. Using the health care professional's expertise would be an important chance to improve focus on quality and costs. 

About the author 


\section{About the author}

Lorette Stammen was born in Belfeld on January the $27^{\text {th }}, 1988$. After a year of Health Sciences in Maastricht she got admitted to the school of Medicine in 2007. After receiving her Master's degree at Maastricht University in 2013 she started her PhD-project at the School of Health Professions Education (SHE) at the Department of Educational Research and Development. In 2015 she decided to combine her research with the family medicine residency program. At the beginning of 2021 she aims to finish residency training and combine clinical work with research.

\section{Academic work}

\section{This thesis}

Stammen LA, Stalmeijer RE, Paternotte E, Oudkerk Pool A, Driessen EW, Scheele F, et al. Training Physicians to Provide High-Value, Cost-Conscious Care: A Systematic Review. JAMA. 2015;314(22):2384-400.

Stammen L, Slootweg I, Stalmeijer R, Janssen L, Stassen L, Scheele F, et al. The Struggle Is Real: How Residents Learn to Provide High-Value, Cost-Conscious Care. Teach Learn Med. 2019;31(4):402-11.

Stammen, Lorette A. MD; Driessen, Erik W. PhD; Notermans, Celine C.V.I.; Scheele, Fedde PhD; Stassen, Laurents P.S. PhD; Stalmeijer, Renée E. PhD How Do Attending Physicians Prepare Residents to Deliver High-Value, Cost-Conscious Care?, Academic Medicine: May 2020 - Volume 95 - Issue 5 - p 764-770

Stammen LA, Janssen LM, Bressers G, Driessen EW, Stassen LP, Stalmeijer RE, et al. A wealth of opportunities: an ethnographic study on learning to deliver high-value, cost-conscious care. medRxiv. 2019:19011916.

Stammen LA, Stalmeijer RE, Stassen LPS, Scheele F, Muris J. 12 tips voor het opleiden in Doelmatige zorg. Submitted for peer review.

\section{Other publications}

Busari, J. O., Stammen, L. A., Gennissen, L. M., \& Moonen, R. M. (2014). Evaluating medical residents as managers of care: a critical appraisal of assessment methods. Advances in medical education and practice, 5, 27-37.

Gennissen, L., Stammen, L., Bueno-de-Mesquita, J., Wieringa, S., \& Busari, J. (2016). Exploring valid and reliable assessment methods for care management education. Leadership in Health Services. 
Stammen, L. (2016). Doelmatige zorg: wat leren we de aios?. Huisarts en wetenschap, 59(10), 455-455.

Peters, S., Clarebout, G., Aertgeerts, B., Michels, N., Pype, P., Stammen, L., \& Roex, A. (2019). Provoking a Conversation Around Students' and Supervisors' Expectations Regarding Workplace Learning. Teaching and Learning in Medicine, 1-12.

Noben, C. Y., Stammen, L. A., Vaassen, S., Haeren, R., Stassen, L., van Mook, W., \& Essers, B. (2020). Discrete choice experiment on educating value-based healthcare. Postgraduate Medical Journal.

Vaassen S, Essers BAB, Stammen LA, Walsh K, Kerssens M, Evers S, Heyligers I, Stassen LPS, Mook van WNKA, Noben CYG. Incorporating Value-based Healthcare Projects in Residency Training: the impact of participation on understanding \& competency development. In preparation

\section{Presentations and Workshops}

AMEE 2013 - Poster Session “" Developing physicians as managers of care: A systematic review of assessment methods."

ICRE 2013 - Short Communication "Developing physicians as managers of care: A systematic review of assessment methods."

Rogano 2014 - Presentation "Putting Stewardship on the Educational Agenda." AMEE 2014 - Poster Session

NVMO 2014 - Research Paper "Putting Stewardship on the Educational Agenda."

AMEE 2015 - Short Communication "Training Physicians to Provide High-Value, CostConscious Care: a Review of the Literature."

Doelmatigheidssymposium 2015 - "Doelmatigheid, hoe leer je dat?"

ICRE 2015 - Poster Session "Training Physicians to Provide High-Value, Cost-Conscious Care: a Review of the Literature."

NVMO 2015 - Research Paper "Training Physicians to Provide High-value, Cost-conscious Care: a Realist Review."

MMV conference 2015 - Workshop Medical Education

AMEE 2016 - Short Communication "The hidden curriculum of high-value, cost-conscious care: a focus group study."

Preventing Overdiagnosis Conference 2016 - "Training physicians to deliver High-Value, Cost-Conscious Care."

AMEE 2017 - Research Paper "The Struggle is real - How residents learn HV3C in the workplace setting."

NVMO 2017 - Onderzoekspaper "Een ware worsteling - het opleiden van arts-assistenten in doelmatigheid. 
AKO conference 2018 - "Opleiden in Doelmatigheid."

Doelmatigheidssymposium OORZON 2018

NVMO 2018 - Ronde tafel "Opleiden in doelmatigheid; de verborgen lessen van de praktijk." Workshop "Conflicthantering”.

MMV Congres 2018 - Workshop "Conflict management \& communicatie".

Pelerin 2018 - "Het trainen van artsen in het leveren van doelmatige zorg".

AMEE 2019 - Research Paper "How residents deal with high-value, cost-conscious caredilemmas: an ethnographic study."

AKO Conference 2019 - "Opleiden in Doelmatige zorg."

Preventing Overdiagnosis Conference 2019

NVMO 2019 - Onderzoekspaper "Opleiding in Doelmatigheid - een kans voor de basisopleiding?" Workshop "Academic Freedom."

AMEE 2020 - Doctoral Report Session "Pursuing High-Value, Cost-Conscious Care - the role of Medical Education."

\section{Other research related activities}

Member of the organizing committee of the departmental conference $1^{\text {st }}$ edition 2015 Member of the Executive board Educational research and development 2015

Member of the organizing committee of the departmental day (O\&O Uitje) 2015

Member of the taskforce social events of the department of educational research and development (de Borrel-commissie) 2015-2019

Member of WDH - Taskforce Expertise Advancement Family Medicine Midden-Limburg

(Werkgroep Deskundigheidsbevordering Huisartsen Midden-Limburg).

2016-to date

Member of the organizing committee of NVMO conference (Lustrum-commissie)

2017-to date

\section{Nominations \& Awards}

Award for Best Scientific Paper Presentation - NVMO conference 2015

Nomination for Best contribution to Academic Advancement Award of Family Medicine

Residents - LOVAH conference 2016

Nomination for Pelerin Award for Talented Researchers: Pitch for kids - Pelerin 2018

Nomination for AMEE Doctoral Report Award - AMEE Conference 2020 


\section{SHE dissertations series}

The SHE Dissertation Series publishes dissertations of $\mathrm{PhD}$ candidates from the School of Health Professions Education (SHE) who defended their PhD theses at Maastricht University. The most recent ones are listed below.

For more information go to: https://she.mumc.maastrichtuniversity.nl Giuliani, M. (19-05-2020) A Critical Review of Global Curriculum Development, Content and Implementation in Oncology

Schreurs, S. (20-03-2020) Selection for medical school; the quest for validity Schumacher, D. (19-03-2020) Resident Sensitive Quality Measures: Defining the Future of Patient-Focused Assessment

Sehlbach, C. (21-02-2020) To be continued.... Supporting physicians' lifelong learning Kikukawa, M. (17-12-2019) The situated nature of validity: Exploring the cultural dependency of evaluating clinical teachers in Japan Kelly, M. (10-12-2019) Body of knowledge. An interpretive inquiry into touch in medical education

Klein, D. (06.11.2019) The performance of medical record review as an instrument for measuring and improving patient safety

Bollen, J. (01.11.2019) Organ donation after euthanasia: medical, legal and ethical considerations

Wagner-Menghin, M. (25-09-2019) Self-regulated learning of history-taking: looking for predictive cues

Wilby, K. (02-07-2019) When numbers become words: Assessors' processing of performance data within OSCEs

Szulewski, A. (20-06-2019) Through the eyes of the physician: Expertise development in resuscitation medicine

McGill, D. (29-05-2019) Supervisor competence as an assessor of medical trainees; Evaluating the validity and quality of supervisor assessments Van Rossum, T. (28-02-2019) Walking the tightrope of training and clinical service; The implementation of time variable medical training 

Pursuing High-Value, Cost-Conscious Care

Dankwoord - Eindelyk Gereel tegen de gewoontes van een lieve Ruld. Toen ik aad dit promole traject begon verllaarde je me log net niet vace gett: kiezer vor 4 yaar conter 'de computer' waarom zau een mens dat gdoen? Maar al snel hwam. e erachter dat ile er veel plezier in had en $j$ vierde de grate en kleine aerwinningen mat me mee. Vood toen het einde een eche worsteling bleek, blefje me hermeren can myn eiger doelen en was je (in de categacie Teugh tove) streng maar ordersteunend Je bent mede de reden dat het proefschrift to stand is gevermen en ik ben dankidaar wor fe steun en schaamteloze trods. Er gaat geen lag voorbij zader dat je my laat bachen en Ik tewonder je energie, je tcornettungsuermagen en ressvast lertrowwen in ezelf in my en in ons. Ik ben by dat it my leven metjou mag dat jis er bent. Met een grde glimlach, een natte kus en een soepsenger kun je de wereld aan en 20 is het moar net?

lieve Papa \& Mama, wat hebben jullie er lang cp moten wachten he? Maar het is of bedankt voor jullic eindelore intersse, bereidwilligveid tat lezen (tat jullie groe spyt) en kntische rragen. Ik hop dat jullie kutrren genieten wan de officiele verdediging, met ons $32 y$ aan $2 y$. Yosine \& marces, myn grcte wyze zussen, waar de deur altyd spen staat, fyn dat jullic myn paranimfen wilten zyn en deze dag met my willen
ueren.

166 
Allerligste veiendinneties; w wllue hebben unhadelyk natumblyk niets bygedragen aan dit proefsotrige en toon was het zonder jullie niet gelukt.

on eertyk te zyn, zonder jullue was er waarschynlyk nog veel meer in het leren nier gelult.

\#em, twelien e Anauk ullie zyn er cittud en vaden feilloos zaken aan en laten veyuer niets onbesproken. Stuk voor stuk zyn ullie moare mensen en iv bewonder jullie veer liadnt. Lieve Gina e Janneke, myn Ibiza-chicks en Solar-beesten en bavend valeybal matties, hoe cen balspelletic kan witarceien tet een echte veiendschop. bedankt daarvoor. lieve $Y_{V}, j e$ verhuist steeds verder by me vandaan maar toon is net altud binnen z minuten weer vertrouwd, ik ben trats ap de uternationde carrier die ic helbt apgebowwd en hab veel geleerd vanjaw perspectief op de zag. Sanne \& Suls, myn Ferrara schatjes, de heeveetheid hermerngen wn eindelecs, jullie waren er altyd en altyd is het leuk Ik ben sipertrats op jallie e jullie gezinneties is Dear Tamara, it has been mare than 10 years sunce you guided me through the NICU in Adelaide and all the hick-ups of life. Who wall have thaught we wauld spend summers in Italy Spain the Netherlands and Australa (with two families on a surf-board) Thank you and fath for weicoming us, teaching Rund has to rub a cheren and sharing yair cheistras-dimer. Zulke veiendinnen zyn gand waard en daar ben it me zeer beuust tan. 
Myn proefsohnift zan nit zen antstaan zader de steun von myn mede-PhD's (en dat waren er veel!) Allemaal bedankt vaor de gerelligheid. de feedlbad en het begrip. Het schrijven von een boelzie is soms best een flinke kus. Er zyn een paar meneen un het byzonder die an dichtby hebben geholpen by myn werkplezier \& onderzaet. Miin 1 ste Hamer: Joerick. Sanne S. Sanne R. koos, Ellen Emmaliple en Andrea. Bedankt voor het atwerpen van myn zavelyk logo, de kook \& didet asonden het sarcasme en tbave all jallie angerauten mening Andrea, je was myn buddy in voor. en tegenspeed s. Ik zal Mumford kJons by de andergaande zon noait vergeten, laten we nog valk genietel van muzick.

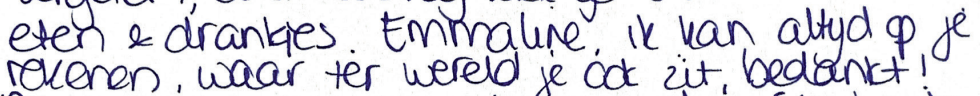
myn tweede namer; luctong Jay, stephanie en Cardin. Thanks for the dinners the advice chocclate and support in the final phase. Liebe Caro, mein. Deutsche Freundin, ich bin so froh Het wai een eer an je paranimf te mogen ayn en ber nosikeds ander de undruk van het werk dat je verzet hebt. Ons borrelclubie: lieve Sums lk ue je rog lopen aver de brug het was liejde o het eerste gercht Bu lai is er geen coreleel en mag alles gezegd werden Heren; bedankt vor allie gezelschap el analyes ion het leren, ik kuk uit haar ullue prometie-jeestjes. Derk, bedankt foor alji leritische vagen en suggesties. ik heb er op gemspperd maar het heett me zek el gehdpen Richting te geven aan dit proyschrift. Serge, attyd valm e stress bestendig. Fyn dat ilk met jou kotsparrer. aver 'ons' onderwerp. Guusje: len feettie is geen teestre als juj er niet by bent. Bedankt dat ji ine wequys hebt gemaakt in de wereld won etnogrefie, al vond ik de momenten dat $x$ myn parther-in-crime was wanneer er ios tebeleven vid ng veel leuker. 
linda e Celine, wat vand ik net leuk on samen met jullue het thema verder te verkernen en e bediscussieiren! I K heb veel van ullie geleed, racl jullie visies op de zarg, hot anderwys als de samen werking an sich. Het is een eer dat ullie beiden 'gegrepen' zyn door het onderwerp en juluie het OP jullie eigen manier integreren in de opleiding Jullie hebben een waardevole byprage geleverd aan dit proejsonrift en hopelyk kruisen anze paden wion in de taekanst rog.

Wat was het een moa congres: Iusteum 2a7 Een top canmissic en nagsteeds een gerellige en inspirerende bende. Emma, je nam my onder je leugels tydens myn eerste studie en hob met veel plezier met ij gewerkt en samen met je niet-gewerkt. liere larsten, we hebben samen een fantastische reis gemaakt en encem veel plevier gemaakt, al is het alweer een tyd geleden, vergeten zol ik het noat. Irene!!k moet al glimlachen als ik aan je denk we hebben ontelbare meters gemaakt un binnen \& butenlang en ve kwamen altyd by de grole thema's wit. le bent wys en geft me altud het gevoel dat ik gelykwaardtg ben, andants ons leeftydsverschl. Renee, Mandele Prouk wot fyn das we samen. met of zander jasjes. nogsteeds aiezelfole chemie hebben als destijds $\rightarrow$ op naar neg reel profecten! 
lieve Anique, voor yu nogeen apart woord ron dank. Het is uoo my enam waarded geweest om' 20 maar' by je bannen te magen wandelen vor advies crer gave en klane dingen. I hedt veel waarde aan is mening enje lebt een talent voor het stellen van de juiste wagen. Annemanie, waar moet k beginnen; je bent een fantastisch mens, zelfshet meest troostelore pannenkoekhuis in the midale of nowherc wordt eln warme plex in jaw an werigheid. Lilian, nicky, Audrey, fianne \& Hemie, bedankt voor jullie ondersteuning en alziende cog ten reactic of hulp was noett ver weg liere nargeiet Desiree, Diana, Danidle \& Marisica, myn OeO lopbaan began by jullie. Bedankt voor jullie langlueige en oprechte interesse.

Tot slat myn team, 3 hoogleraren en 1 co-pronator hoe verzin je her? laurents ane sollicitatic/kennismaking vond plaats tyjdens de lunch von het nvmo-cengres en toen al toonde je veel vertrouwen in my en dit team. Gedurende de jaren ben fedat vertraumen noat verceren en hebje my geseund in het combireren van anderzoek en myn eigen medische veridgopleiding.

Feade anze kernismaking was odk nev erg conventicreel, als ik hat me goed herinner was net ergens in een Praagse kroeg waar y verkandigde dat ji een idee had voor anderzoec 'dat er echt toe deed'. Ik geef toe, ik heb geaarzeld, maar ik was shel gegreper clock het anderuerp en bÿbeharende dilemma's. Je hebt het vermoger om alles als 'leuk' en spamend' te kaderen, je rag altyd het postieve un my en in ans orderzeek bedankt! lieve trik, de man van de plaming? By herrialung zei ci dat het myn profschrift was maar waar nodig trok $\dot{ }$ toon aan de teugels (shauld i) 
Ye bood attyd 9 andere pletken nieuve ruinte ye hebt me (althars dat hoop ik) gogeleid tot een zelfstandig onderzeker en il hoop doar nag lang gebruik van te maken. last but not least Renee. Je was myn mentor in jaar 1 Geneeskunde en snel dawen myn baas by PE. Daar werd myn intersse gewett voor medisch, onderwys en onderzak Volgens my is er geen letter in dit prof sohnift nio via jur gegaan, z bent gedegen, precres en streeft ally a naar het allerbeste. Je was veel meer dan een dagelyks begeleider en helot me o meer vakken begeleidt dan alleen dit proefschrift, bedankt is echt een understatement. 
